\title{
Catalytic Enantioselective Synthesis of Difluorinated Alkyl Bromides
}

\author{
Mark D. Levin ${ }^{\dagger}$ John M. Ovian ${ }^{\dagger}$, Jacquelyne A. Read ${ }^{\dagger P}$, Matthew S. Sigman ${ }^{\text {P* }}$, and Eric N. Jacobsen ${ }^{\dagger *}$ \\ ${ }^{\dagger}$ Department of Chemistry and Chemical Biology, Harvard University, 12 Oxford Street, Cambridge, Massachusetts \\ 02138, United States \\ PDepartment of Chemistry, University of Utah, 315 South 1400 East, Salt Lake City, UT 84112, United States \\ \$These authors contributed equally
}

\section{Experimental Supporting Information}

General Considerations

Comments regarding the origins of commercially available starting materials, purification of solvents, and spectroscopic techniques.

Synthesis of Vinyl Bromide Substrates

Procedures for the preparation of substrates and characterization information.

Synthesis of Chiral Catalysts

Procedure for the preparation of catalysts and characterization information. Catalyst structure-activity relationship study.

Select optimization data and crude reaction mixture NMR spectra.

General Procedure for Oxidative Rearrangement

General procedure for synthesis, isolation procedures, and characterization information for alkyl halides.

\section{Derivatizations of Products of Oxidative Rearrangement}

Procedures for the synthesis, isolation, and characterization of derivatives of the secondary alkyl bromides.

X-ray Crystallography

Details and data for the determination of the absolute configuration.

References

\section{General Considerations:}

General:

All reactions for the preparation of substrates were performed in standard, dry glassware fitted with rubber septa under an inert atmosphere of nitrogen unless otherwise described. All difluorination reactions were performed in low density polyethylene tubes sealed with a low-density polyethylene cap under an atmosphere of air. Reported concentrations refer to solution volumes at room temperature. Concentration of organic solutions under reduced pressure was performed using house vacuum (ca. $40 \mathrm{~mm} \mathrm{Hg}$ ) at $30^{\circ} \mathrm{C}$. Column chromatography was performed with SiliaFlash P60 (230-400 mesh, SiliCycle). Thin layer chromatography (TLC) was used for reaction monitoring and product detection was performed using pre-coated glass plates covered with $0.20 \mathrm{~mm}$ silica gel with fluorescent indicator; plates were visualized by exposure to UV light $\left(\lambda_{\mathrm{ex}}=254 \mathrm{~nm}\right)$ or by staining with potassium permanganate or ninhydrin.

CAUTION: Pyridine $9 \mathrm{HF}$ is a corrosive and toxic substance that will etch glassware. Safe handling can be conducted with plastic syringes and metal needles, with $\mathrm{NaHCO}_{3}$ (aq.) or $\mathrm{NaOH}$ (aq.) employed to quench excess $\mathrm{HF}$. Though 
reactions should not be conducted in glassware when employing pyridine $9 \mathrm{HF}$, glassware may be used to quench reactions provided sufficient quantities of base are present. Always handle pyridine 9 HF while wearing gloves and in a fumehood. As a precautionary measure, have calcium gluconate gel nearby and apply immediately and liberally on skin exposed to HF.

Materials. Reagents were purchased in reagent grade from commercial suppliers and used as received, unless otherwise described. Anhydrous solvents (dioxane, dichloromethane, N,N-dimethylformamide, and tetrahydrofuran) were prepared by passing the solvent through an activated alumina column.

Instrumentation. Proton nuclear magnetic resonance (1H NMR) spectra were recorded on a Varian Mercury-400 or an Inova-500 spectrometer, are reported in parts per million downfield from tetramethylsilane, and are referenced to the residual protium resonances of the NMR solvent (CDCl3: $\left.7.26\left[\mathrm{CHCl}_{3}\right]\right)$. Proton-decoupled carbon-13 nuclear magnetic resonance (13C $\{1 \mathrm{H}\} \mathrm{NMR}$ ) spectra were recorded on an Inova-500 spectrometer, are reported in parts per million downfield from tetramethylsilane, and are referenced to the carbon resonances of the NMR solvent $\left(\mathrm{CDCl}_{3}\right.$ : 77.3). Chemical shifts for fluorine-19 nuclear magnetic resonance (19F NMR) were recorded on an Inova-500 spectrometer and are reported in parts per million downfield from chlorotrifluoromethane and are referenced to the fluorine resonance of chlorotrifluoromethane $(\delta=0)$. Data are represented as follows: chemical shift, multiplicity (br $=$ broad, $\mathrm{s}=$ singlet, $\mathrm{d}=$ doublet, $\mathrm{t}=$ triplet, $\mathrm{q}=$ quartet, quin $=$ quintet, sext $=$ sextet, sept $=$ septet, $\mathrm{m}=$ multiplet), coupling constants in Hertz (Hz), integration. High resolution mass spectrometric data were obtained on a Thermo qExactive Plus coupled with an Ultimate 3000 uHPLC (ESI) or GC (EI). GC analysis was performed using an Agilent 7890A GC system using commercially available columns. Chiral HPLC analysis was performed using an Agilent 1200 series quaternary HPLC system using commercially available CHIRALCEL analytical columns (4.6 x $250 \mathrm{~mm})$.

\section{Synthesis of Vinyl Bromide Substrates and Their Precursors}

\section{General Procedure A}

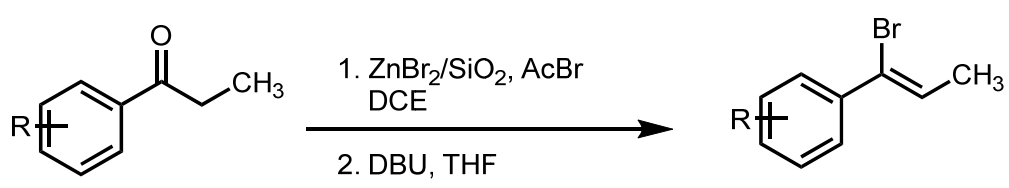

The following procedure was adapted from one reported by Legault and coworker. ${ }^{1}$ To a 2-dram vial equipped with a septum cap and stir bar was added the propiophenone (1 equiv) and dichloroethane $(0.5 \mathrm{M})$. Acetyl bromide ( 8 equiv) was added all at once via syringe, followed by $\mathrm{ZnBr} / \mathrm{SiO}_{2}(1 \mathrm{~g} / \mathrm{mmol}$ substrate) while vigorously stirring. The reaction was allowed to proceed overnight. After this time, the reaction was filtered, washing with DCM, and quenched with $\mathrm{NaHCO}_{3}$. The solution was then transferred to a separatory funnel, diluted with DCM and additional bicarbonate. The organic layer was separated and the aqueous layer extracted with DCM thrice. The combined organics were then washed with $\mathrm{NaHCO}_{3}, \mathrm{H}_{2} \mathrm{O}$, and brine, and subsequently dried over $\mathrm{Na}_{2} \mathrm{SO}_{4}$, filtered, and concentrated in vacuo. The crude material was then purified by column chromatography (Hexanes: $\mathrm{Et}_{2} \mathrm{O}$ ) to afford a mixture of the vinyl bromide and the gem-dibromoalkane.

In a vial equipped with a stir bar and septum cap was added the material from the previous step and THF (1M). To the stirred solution was added DBU (1.5 equiv relative to gem-dibromoalkane), and the reaction was allowed to proceed overnight. After this time, the mixture was diluted with $\mathrm{Et}_{2} \mathrm{O}$ and $\mathrm{H}_{2} \mathrm{O}$. The organic layer was separated and the aqueous layer extracted with $\mathrm{Et}_{2} \mathrm{O}$. The combined organics were then washed with $\mathrm{H}_{2} \mathrm{O}$ and brine, dried over $\mathrm{Na}_{2} \mathrm{SO}_{4}$, filtered, and concentrated in vacuo. The crude material was then purified by column chromatography (Hexanes: $\mathrm{Et}_{2} \mathrm{O}$ ) to afford the desired product. 


\section{General Procedure B}

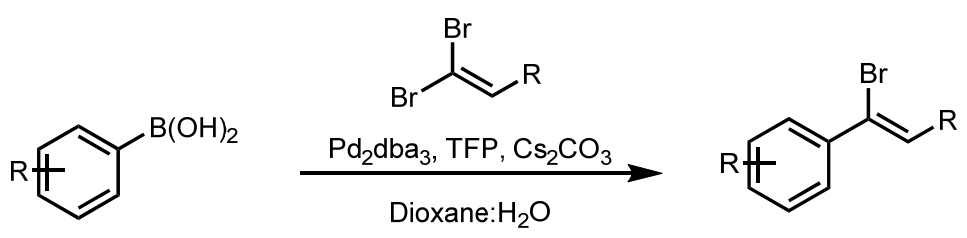

The following procedure was adapted from one reported by Paraja, et. al. ${ }^{2}$ To a vial equipped with a stir var and septum cap was added the boronic acid ( 1 equiv), $\mathrm{Pd}_{2} \mathrm{dba}_{3}$ ( 0.025 equiv), and tri(2-furyl)phosphine ( 0.15 equiv). The vial was evacuated and backfilled thrice with $\mathrm{N}_{2}$. To a separate vial equipped with a septum cap was added the gemdibromoalkene (1.2 equiv). This was evacuated and backfilled thrice with $\mathrm{N}_{2}$, followed by addition of dioxane $(0.25 \mathrm{M}$ in boronic acid). The solution was then sparged with $\mathrm{N}_{2}$ for 5 minutes. To a third vial equipped with a septum cap was added $\mathrm{Cs}_{2} \mathrm{CO}_{3}$. This vial was evacuated and backfilled thrice with $\mathrm{N}_{2}$, followed by addition of $\mathrm{H}_{2} \mathrm{O}$ (1M in boronic acid). The solution was then sparged with $\mathrm{N}_{2}$ for 5 minutes. After this time, the alkene in dioxane and the aqueous $\mathrm{Cs}_{2} \mathrm{CO}_{3}$ were added simultaneously dropwise to the vial containing the boronic acid. This mixture was allowed to stir vigorously overnight. After this time, the mixture was filtered through celite, washing with EtOAc. The organic layer was then washed with brine thrice, dried over $\mathrm{Na}_{2} \mathrm{SO}_{4}$, filtered, and concentrated in vacuo. The crude material was

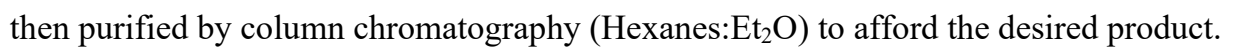<smiles>C/C=C(\Br)c1cccc([N+](=O)[O-])c1</smiles>

2a, (Z)-1-(1-bromoprop-1-en-1-yl)-3-nitrobenzene. Prepared according to General Procedure A from 3'nitropropiophenone $(896 \mathrm{mg}, 5 \mathrm{mmol}$ ) as a clear, colorless oil, which solidified upon storage (900 $\mathrm{mg}$, 74\% yield).

${ }^{1} \mathrm{H}$ NMR $\left(500 \mathrm{MHz}, \mathrm{CDCl}_{3}\right) \delta 8.38(\mathrm{~s}, 0 \mathrm{H}), 8.14(\mathrm{ddd}, J=8.2,2.4,1.0 \mathrm{~Hz}, 1 \mathrm{H}), 7.85$ (ddd, $\left.J=7.8,1.9,1.0 \mathrm{~Hz}, 1 \mathrm{H}\right)$, $7.51(\mathrm{t}, J=8.0 \mathrm{~Hz}, 1 \mathrm{H}), 6.45(\mathrm{q}, J=6.6 \mathrm{~Hz}, 1 \mathrm{H}), 2.00(\mathrm{~d}, J=6.6 \mathrm{~Hz}, 3 \mathrm{H})$.

${ }^{13} \mathrm{C}$ NMR $\left(126 \mathrm{MHz}, \mathrm{CDCl}_{3}\right) \delta 148.14,141.61,133.21,129.23,123.72,122.85,122.28,18.30$.

HRMS (ESI): for $\mathrm{C}_{9} \mathrm{H}_{9} \mathrm{BrNO}_{2}$, [M+H] $]^{+}$calculated $\mathrm{m} / z=241.9810$ and 243.9791, found $\mathrm{m} / z=241.9811$ and 243.9790 .

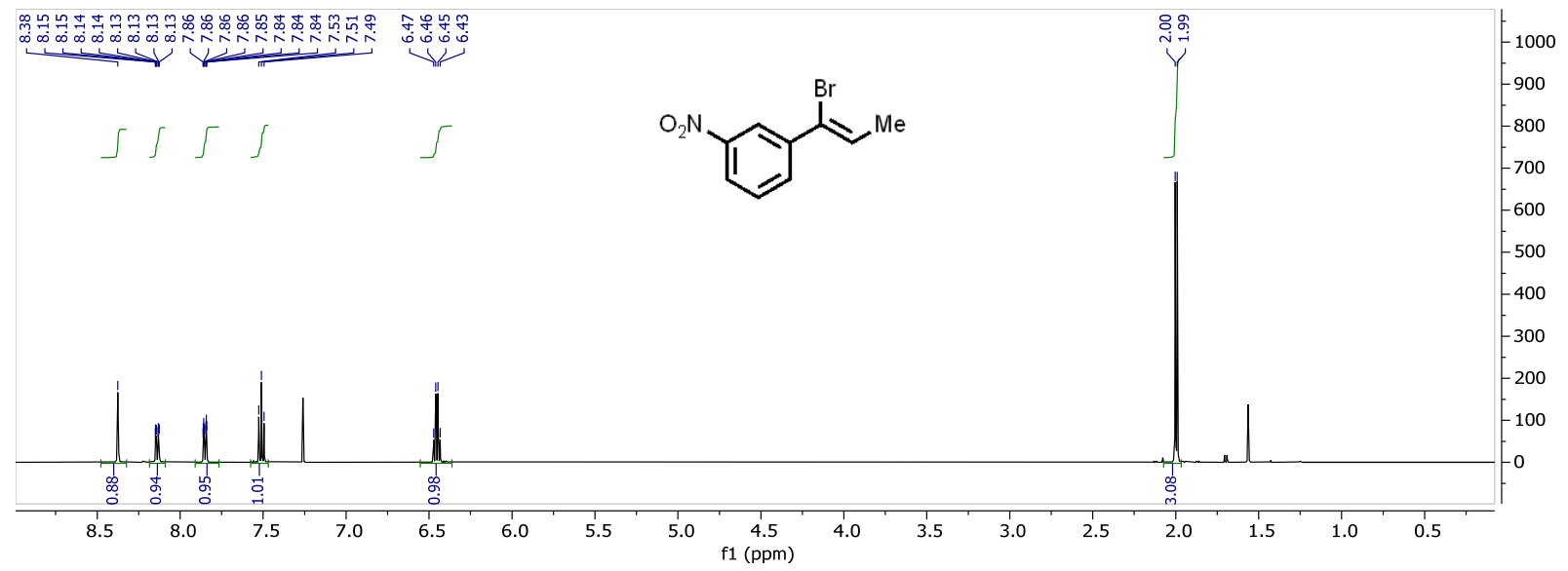




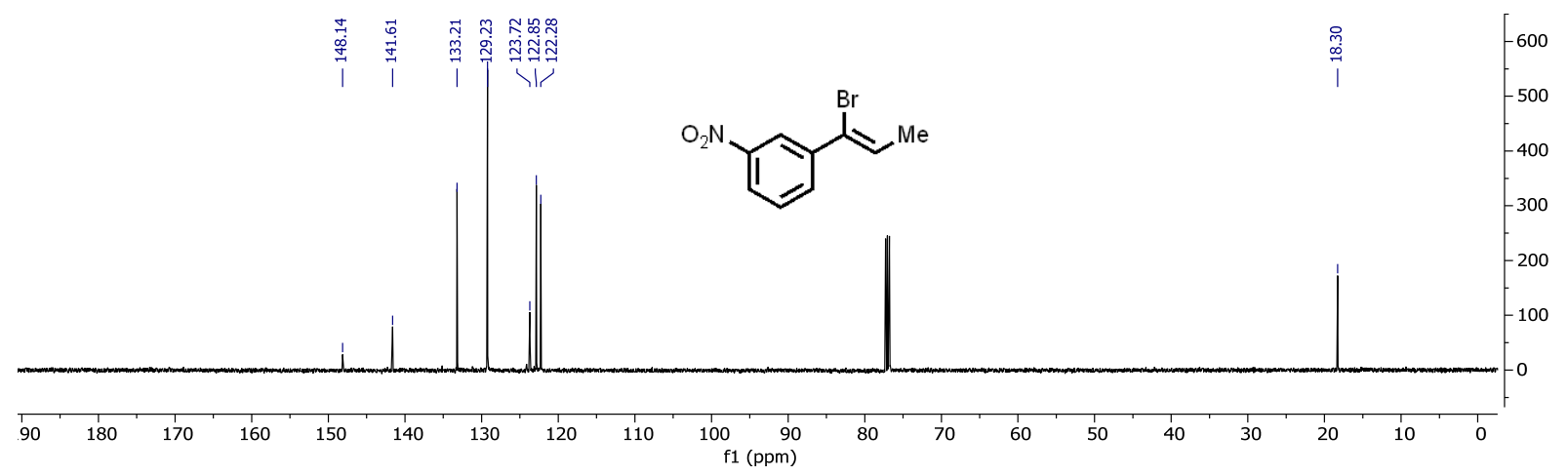<smiles>C/C=C/c1cccc(C(=O)OCC)c1</smiles>

2c, (Z)-ethyl 3-(1-bromobut-1-en-1-yl)benzoate. Prepared according to General Procedure B from 3(ethoxycarbonyl)phenylboronic acid (970 $\mathrm{mg}, 5 \mathrm{mmol}$ ) as a clear, orange oil (396 $\mathrm{mg}, 28 \%$ yield).

${ }^{1} \mathrm{H}$ NMR $\left(500 \mathrm{MHz}, \mathrm{CDCl}_{3}\right) \delta 8.21-8.18(\mathrm{~m}, 1 \mathrm{H}), 7.97$ (ddd, $\left.J=7.7,1.7,1.1 \mathrm{~Hz}, 1 \mathrm{H}\right), 7.73$ (ddd, $J=7.8,2.0,1.1$ $\mathrm{Hz}, 1 \mathrm{H}), 7.42(\mathrm{td}, J=7.8,0.6 \mathrm{~Hz}, 1 \mathrm{H}), 6.29(\mathrm{t}, J=6.8 \mathrm{~Hz}, 1 \mathrm{H}), 4.41(\mathrm{q}, J=7.1 \mathrm{~Hz}, 2 \mathrm{H}), 2.46-2.36(\mathrm{~m}, 2 \mathrm{H}), 1.42$ (td, $J=7.1,0.7 \mathrm{~Hz}, 3 \mathrm{H}), 1.14$ (td, $J=7.6,0.7 \mathrm{~Hz}, 3 \mathrm{H})$.

${ }^{13} \mathrm{C}$ NMR $\left(126 \mathrm{MHz}, \mathrm{CDCl}_{3}\right) \delta 166.17,140.31,134.29,131.87,130.58,129.19,128.38,128.29,123.61,61.14$, 26.04, 14.35, 12.80 .

HRMS (ESI): for $\mathrm{C}_{13} \mathrm{H}_{16} \mathrm{BrO}_{2},[\mathrm{M}+\mathrm{H}]^{+}$calculated $\mathrm{m} / z=283.0328$ and 285.0308 , found $\mathrm{m} / z=283.0327$ and 285.0305 .

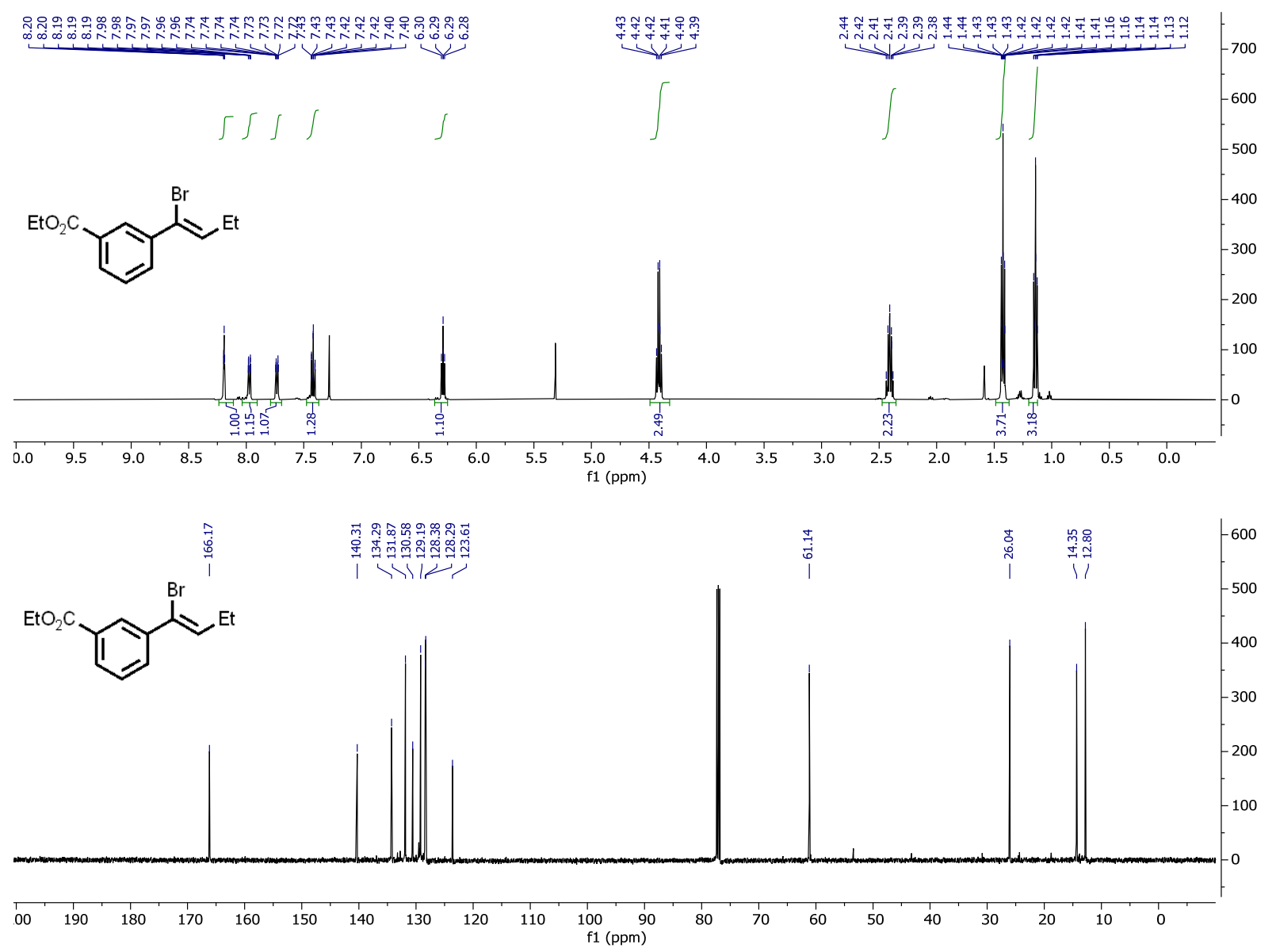


<smiles>CCOC(=O)c1cccc(C(Br)=CC(C)(C)C)c1</smiles>

2d, (Z)-ethyl 3-(1-bromohex-1-en-1-yl)benzoate. Prepared according to General Procedure B from 3(ethoxycarbonyl)phenylboronic acid (194 mg, $1 \mathrm{mmol})$ as a clear, yellow oil (155 mg, 49\% yield).

${ }^{1} \mathrm{H}$ NMR $\left(500 \mathrm{MHz}, \mathrm{CDCl}_{3}\right) \delta 8.17(\mathrm{t}, J=1.9 \mathrm{~Hz}, 1 \mathrm{H}), 7.95(\mathrm{dt}, J=7.7,1.3 \mathrm{~Hz}, 1 \mathrm{H}), 7.73-7.70(\mathrm{~m}, 1 \mathrm{H}), 7.40(\mathrm{t}, J$ $=7.8 \mathrm{~Hz}, 1 \mathrm{H}), 6.28(\mathrm{t}, J=6.9 \mathrm{~Hz}, 1 \mathrm{H}), 4.40(\mathrm{q}, J=7.2 \mathrm{~Hz}, 2 \mathrm{H}), 2.38(\mathrm{q}, J=7.2 \mathrm{~Hz}, 2 \mathrm{H}), 1.55-1.47(\mathrm{~m}, 2 \mathrm{H}), 1.41$ (t, $J=7.1 \mathrm{~Hz}, 5 \mathrm{H}), 0.95(\mathrm{t}, J=7.2 \mathrm{~Hz}, 3 \mathrm{H})$.

${ }^{13} \mathrm{C}$ NMR $\left(126 \mathrm{MHz}, \mathrm{CDCl}_{3}\right) \delta 166.23,140.41,133.04,131.89,130.57,129.18,128.40,128.30,124.04,61.16$, $32.31,30.50,22.42,14.35,13.94$.

HRMS (ESI): for $\mathrm{C}_{15} \mathrm{H}_{20} \mathrm{BrO}_{2},[\mathrm{M}+\mathrm{H}]^{+}$calculated $m / z=311.0641$ and 313.0621, found $m / z=311.0640$ and 313.0618 .

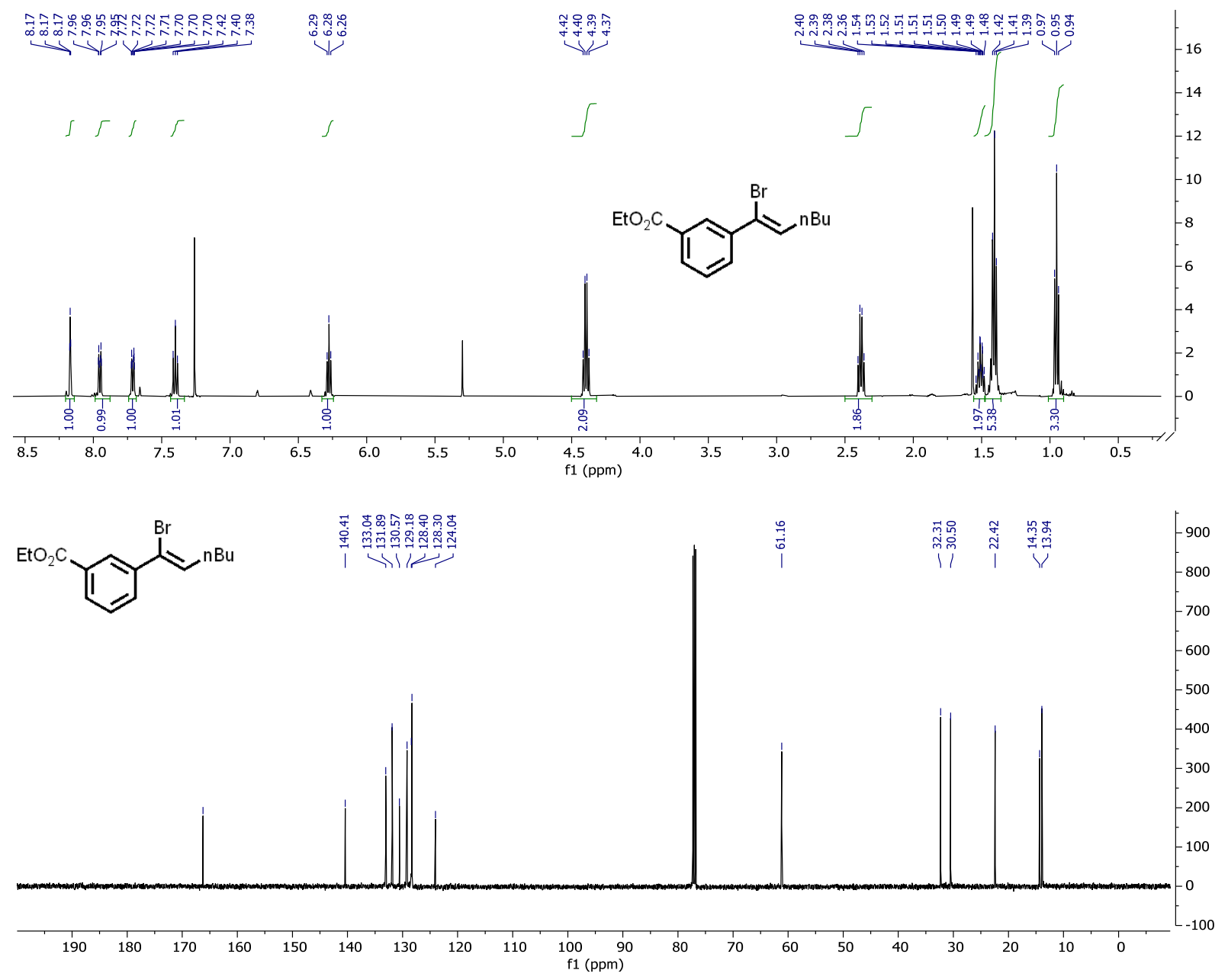


$\underbrace{}_{3}$

2e, (Z)-1-(1-bromoprop-1-en-1-yl)-3-(trifluoromethyl)benzene. Prepared according to General Procedure A from 3'(trifluoromethyl)propiophenone $(606 \mathrm{mg}, 3 \mathrm{mmol}$ ) as a clear, colorless oil $(580 \mathrm{mg}, 73 \%)$.

${ }^{1} \mathrm{H}$ NMR $\left(500 \mathrm{MHz}, \mathrm{CDCl}_{3}\right) \delta 7.78(\mathrm{~s}, 1 \mathrm{H}), 7.72(\mathrm{dt}, J=7.8,1.5 \mathrm{~Hz}, 1 \mathrm{H}), 7.58-7.53(\mathrm{~m}, 1 \mathrm{H}), 7.49-7.44(\mathrm{~m}, 1 \mathrm{H})$, $6.38(\mathrm{q}, J=6.6 \mathrm{~Hz}, 1 \mathrm{H}), 1.99(\mathrm{~d}, J=6.6 \mathrm{~Hz}, 3 \mathrm{H})$.

${ }^{13} \mathrm{C}$ NMR $\left(126 \mathrm{MHz}, \mathrm{CDCl}_{3}\right) \delta 140.83,130.98(\mathrm{~d}, J=32.6 \mathrm{~Hz}), 130.67,128.76,128.11,124.83(\mathrm{q}, J=4.1 \mathrm{~Hz})$, 124.23 (q, $J=3.9 \mathrm{~Hz}), 121.76(\mathrm{~d}, J=272.4 \mathrm{~Hz}), 18.18$.

${ }^{19} \mathrm{~F}$ NMR (471 MHz, Chloroform- $d$ ) $\delta-65.28$.

HRMS (EI): for $\mathrm{C}_{10} \mathrm{H}_{8} \mathrm{BrF}_{3},[\mathrm{M}]^{+}$calculated $m / z=263.9756$ and 265.9736, found $m / z=263.9750$ and 265.9729 .
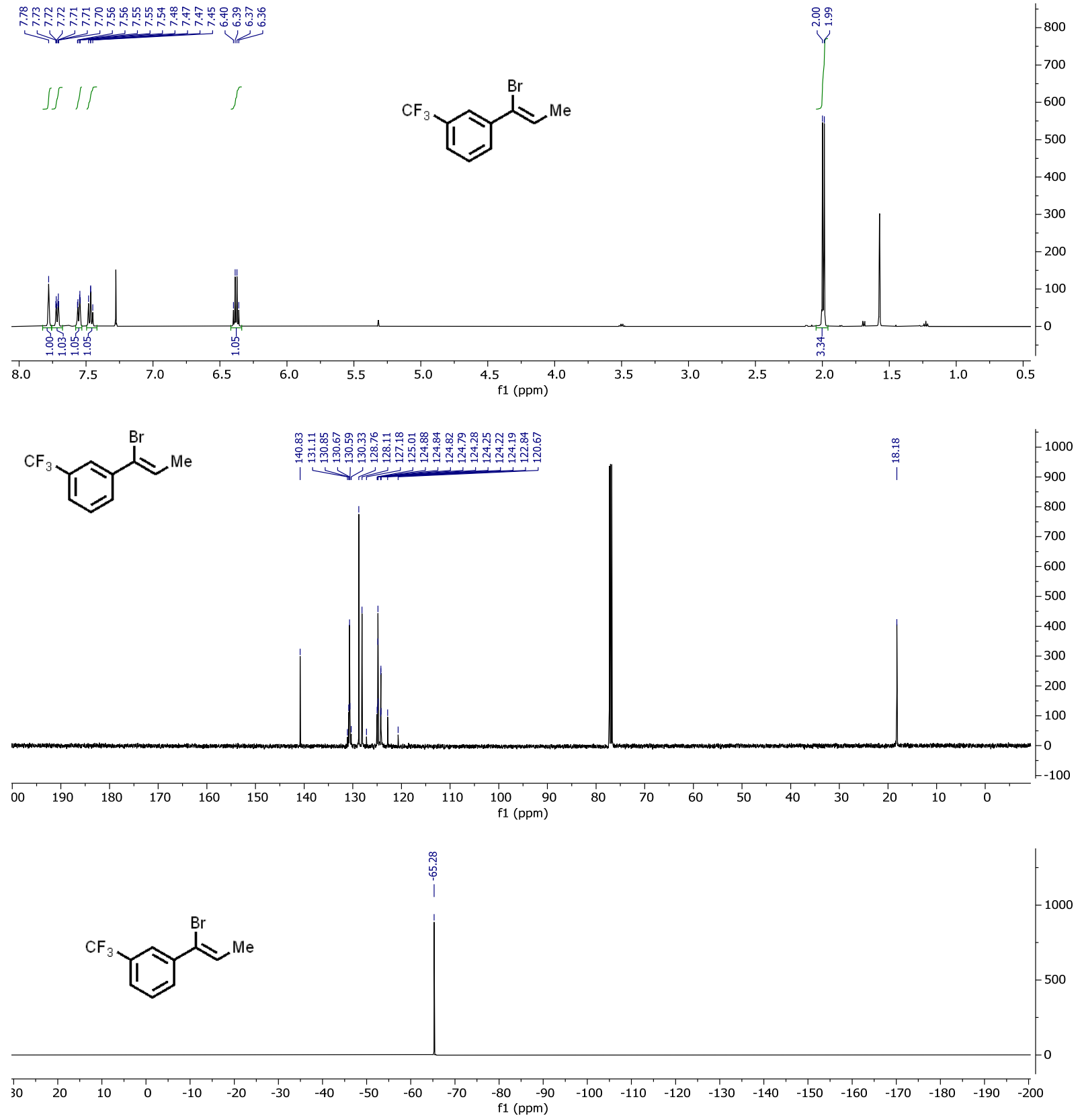
<smiles>CC(C)(C)c1cccc(/C(Br)=C/C#N)c1</smiles>

2f, (Z)-3-(1-bromohex-1-en-1-yl)benzonitrile. Prepared according to General Procedure B from 3cyanophenylboronic acid ( $441 \mathrm{mg}, 3 \mathrm{mmol}$ ) as a clear, orange oil (446 mg, 56\% yield).

${ }^{1} \mathrm{H}$ NMR $\left(600 \mathrm{MHz}, \mathrm{CDCl}_{3}\right) \delta 7.80(\mathrm{~s}, 1 \mathrm{H}), 7.74(\mathrm{~d}, J=8.0 \mathrm{~Hz}, 1 \mathrm{H}), 7.55(\mathrm{~d}, J=7.7 \mathrm{~Hz}, 1 \mathrm{H}), 7.43(\mathrm{t}, J=7.8 \mathrm{~Hz}$, $1 \mathrm{H}), 6.28(\mathrm{t}, J=6.9 \mathrm{~Hz}, 1 \mathrm{H}), 2.38(\mathrm{q}, J=7.2 \mathrm{~Hz}, 3 \mathrm{H}), 1.50(\mathrm{p}, J=7.5 \mathrm{~Hz}, 3 \mathrm{H}), 1.45-1.36(\mathrm{~m}, 3 \mathrm{H}), 0.95(\mathrm{t}, J=7.3$ $\mathrm{Hz}, 5 \mathrm{H})$.

${ }^{13} \mathrm{C}$ NMR $\left(126 \mathrm{MHz}, \mathrm{CDCl}_{3}\right) \delta 141.24,134.42,131.68,131.49,129.12,122.53,118.45,112.56,32.34,30.38,22.39$, 13.91.

HRMS (ESI): for $\mathrm{C}_{13} \mathrm{H}_{15} \mathrm{BrN}$, [M+H] $]^{+}$calculated $\mathrm{m} / z=264.0382$ and 266.0362 , found $\mathrm{m} / z=264.0381$ and 266.0360 .
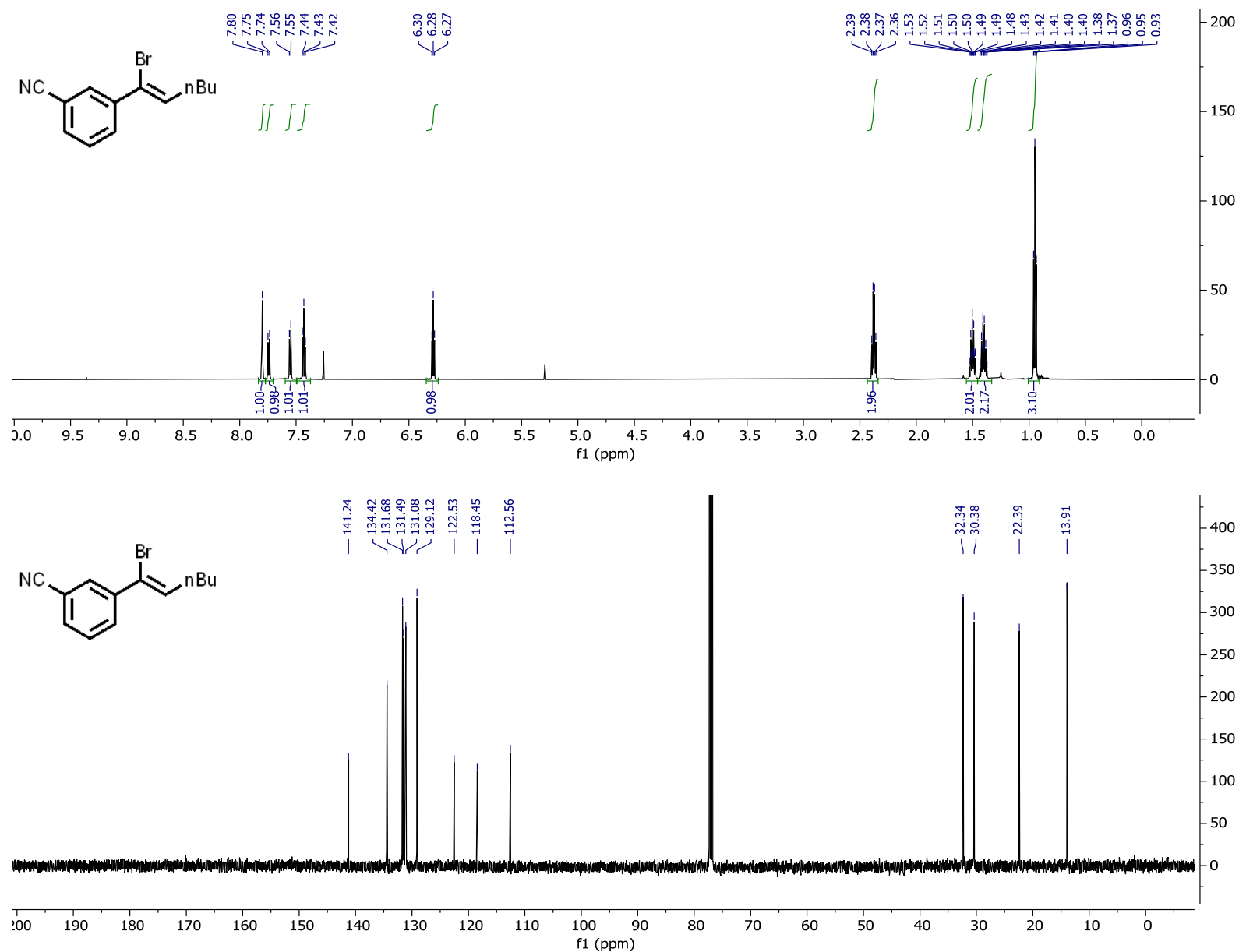
<smiles>CC/C=C(\Br)c1cccc(Br)c1</smiles>

2g, (Z)-1-bromo-3-(1-bromobut-1-en-1-yl)benzene. Prepared according to General Procedure A from 1-(3bromophenyl)butan-1-one (227 mg, $1 \mathrm{mmol})$ as a clear, colorless oil $(220 \mathrm{mg}, 76 \%)$.

${ }^{1} \mathrm{H}$ NMR $\left(500 \mathrm{MHz}, \mathrm{CDCl}_{3}\right) \delta 7.67(\mathrm{~s}, 0 \mathrm{H}), 7.45(\mathrm{ddd}, J=7.9,1.8,1.0 \mathrm{~Hz}, 1 \mathrm{H}), 7.41$ (ddd, $\left.J=8.0,1.9,1.0 \mathrm{~Hz}, 1 \mathrm{H}\right)$, $7.19(\mathrm{t}, J=7.9 \mathrm{~Hz}, 1 \mathrm{H}), 6.22(\mathrm{t}, J=6.9 \mathrm{~Hz}, 1 \mathrm{H}), 2.37(\mathrm{qd}, J=7.6,6.9 \mathrm{~Hz}, 2 \mathrm{H}), 1.11(\mathrm{t}, J=7.6 \mathrm{~Hz}, 3 \mathrm{H})$.

${ }^{13} \mathrm{C}$ NMR $\left(126 \mathrm{MHz}, \mathrm{CDCl}_{3}\right) \delta 141.97,134.57,131.16,130.46,129.68,126.19,122.96,122.28,26.05,12.83$.

HRMS (EI): for $\mathrm{C}_{10} \mathrm{H}_{10} \mathrm{Br}_{2}$, [M] $]^{+}$calculated $\mathrm{m} / z=287.9144$ and 289.9123 and 291.9103, found $\mathrm{m} / z=287.9143$ and 289.9121 and 291.9102.

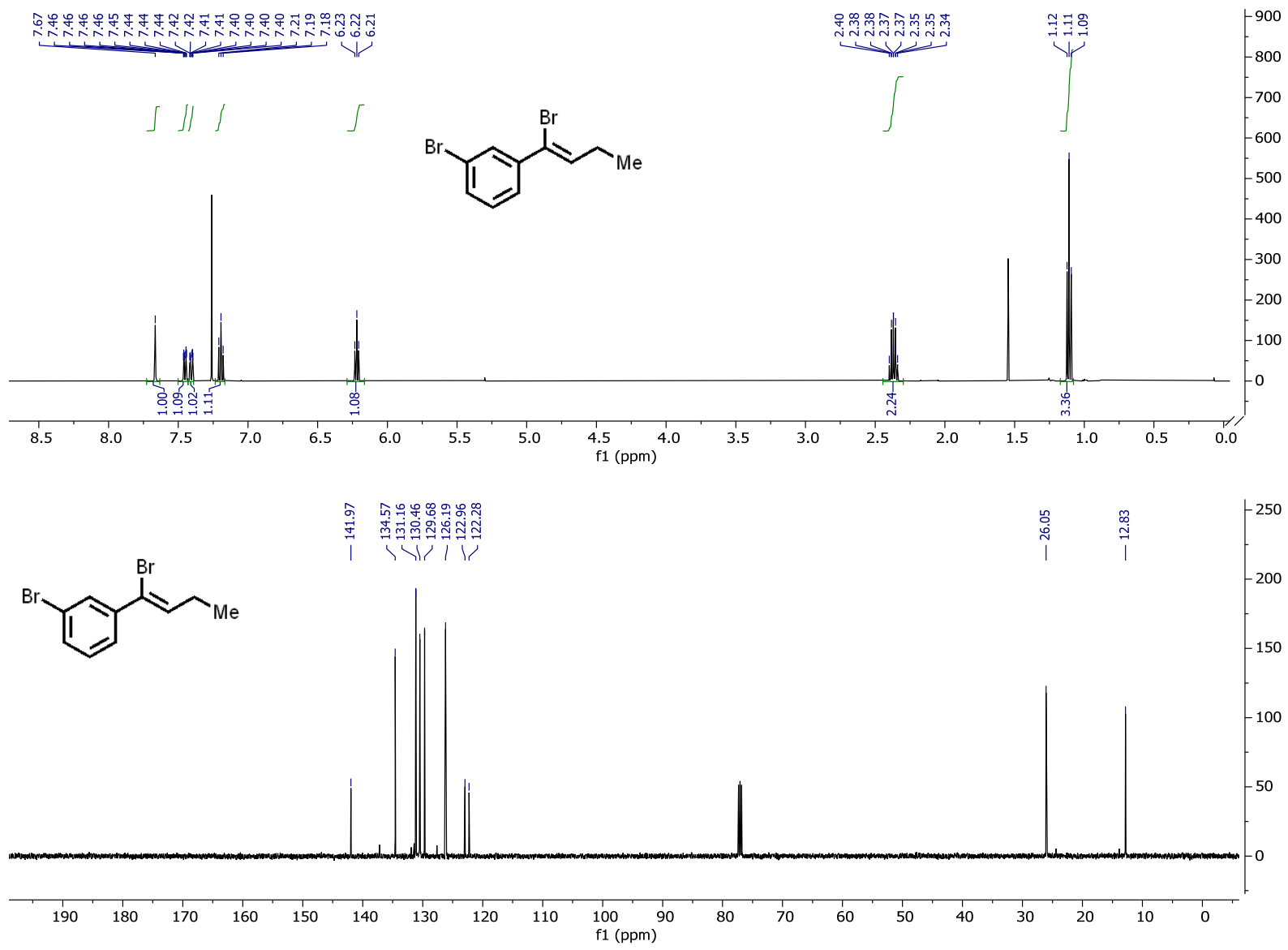


$\underbrace{i P r}$

2h, (Z)-1-bromo-3-(1-bromo-3-methylbut-1-en-1-yl)benzene. Prepared according to General Procedure A from 1-(3bromophenyl)-3-methylbutan-1-one (241 mg, $1 \mathrm{mmol})$ as a clear, colorless oil (240 $\mathrm{mg}, 79 \%)$.

${ }^{1} \mathrm{H}$ NMR $\left(500 \mathrm{MHz}, \mathrm{CDCl}_{3}\right) \delta 7.66(\mathrm{t}, J=1.9 \mathrm{~Hz}, 1 \mathrm{H}), 7.43(\mathrm{dddd}, J=22.0,8.0,1.9,1.0 \mathrm{~Hz}, 2 \mathrm{H}), 7.19(\mathrm{t}, J=7.9$ $\mathrm{Hz}, 1 \mathrm{H}), 6.03(\mathrm{~d}, J=8.7 \mathrm{~Hz}, 1 \mathrm{H}), 2.88(\mathrm{dp}, J=8.8,6.7 \mathrm{~Hz}, 1 \mathrm{H}), 1.10(\mathrm{~d}, J=6.7 \mathrm{~Hz}, 6 \mathrm{H})$.

${ }^{13} \mathrm{C}$ NMR $\left(126 \mathrm{MHz}, \mathrm{CDCl}_{3}\right) \delta 141.99,139.76,131.15,130.49,129.65,126.28,122.25,121.21,32.21,21.68(\mathrm{~d}, J=$ $2.1 \mathrm{~Hz})$.

HRMS (EI): for $\mathrm{C}_{11} \mathrm{H}_{12} \mathrm{Br}_{2},[\mathrm{M}]^{+}$calculated $\mathrm{m} / z=301.9300$ and 303.9280 and 305.9259 , found $\mathrm{m} / z=301.9290$ and 303.9268 and 305.9249 .
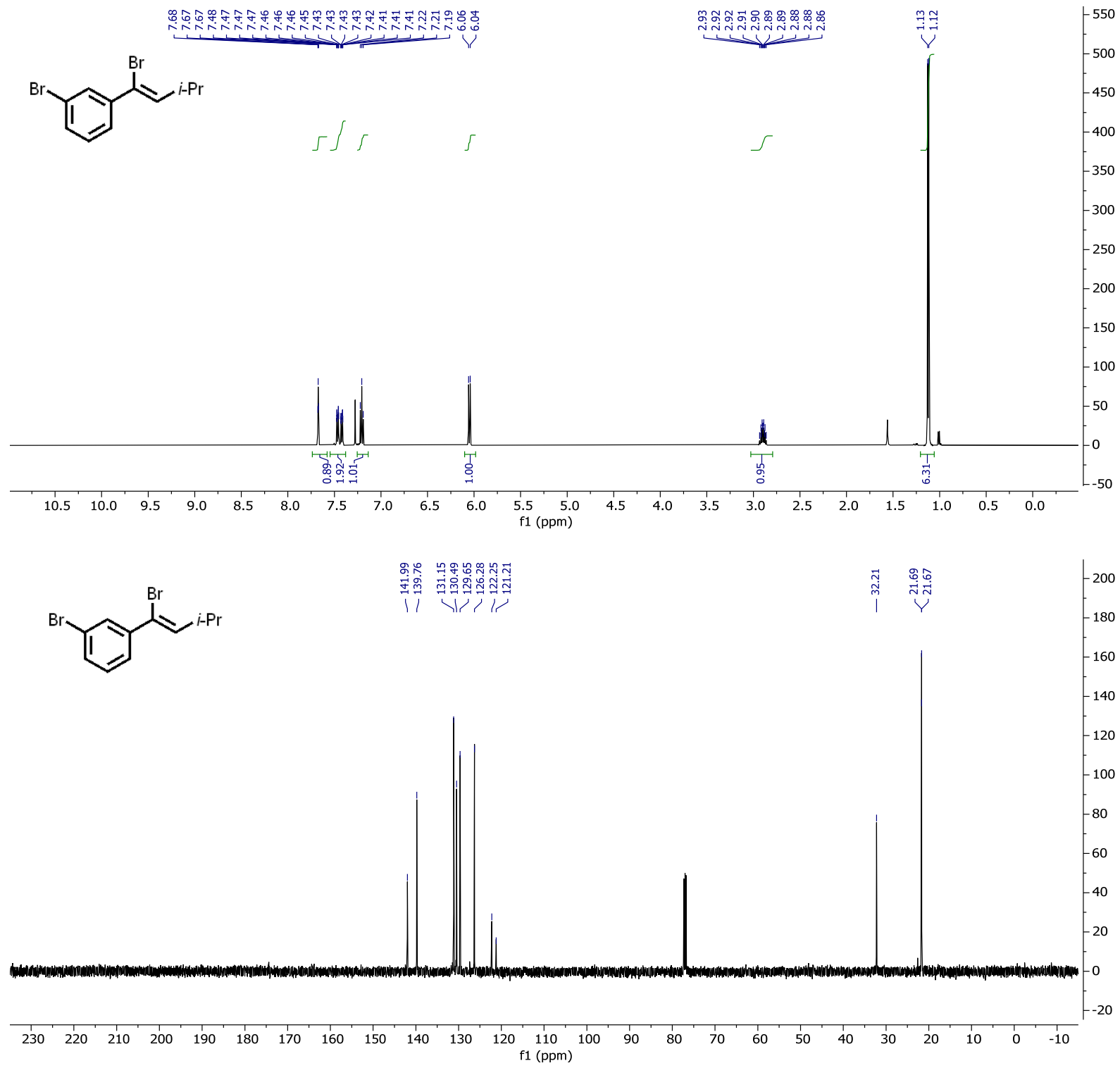
<smiles>CC/C=C(\Br)c1ccc([N+](=O)[O-])cc1</smiles>

2i, (Z)-1-(1-bromobut-1-en-1-yl)-4-nitrobenzene. Prepared according to General Procedure B from 4nitrophenylboronic acid (166 mg, $1 \mathrm{mmol}$ ) as an orange solid ( $80 \mathrm{mg}, 31 \%$ yield).

${ }^{1} \mathrm{H}$ NMR $\left(500 \mathrm{MHz}, \mathrm{CDCl}_{3}\right) \delta 8.20(\mathrm{~d}, J=8.9 \mathrm{~Hz}, 2 \mathrm{H}), 7.71(\mathrm{~d}, J=8.9 \mathrm{~Hz}, 2 \mathrm{H}), 6.42(\mathrm{t}, J=6.9 \mathrm{~Hz}, 1 \mathrm{H}), 2.51-$ $2.36(\mathrm{~m}, 2 \mathrm{H}), 1.16(\mathrm{t}, J=7.6 \mathrm{~Hz}, 3 \mathrm{H})$.

${ }^{13} \mathrm{C}$ NMR (126 MHz, $\left.\mathrm{CDCl}_{3}\right) \delta 147.35,145.86,137.16,128.17,123.52,122.36,26.27,12.63$.

HRMS (ESI): for $\mathrm{C}_{10} \mathrm{H}_{11} \mathrm{BrNO}_{2},[\mathrm{M}+\mathrm{H}]^{+}$calculated $\mathrm{m} / \mathrm{z}=255.9968$ and 257.9947 , found $\mathrm{m} / \mathrm{z}=255.9967$ and 257.9946.
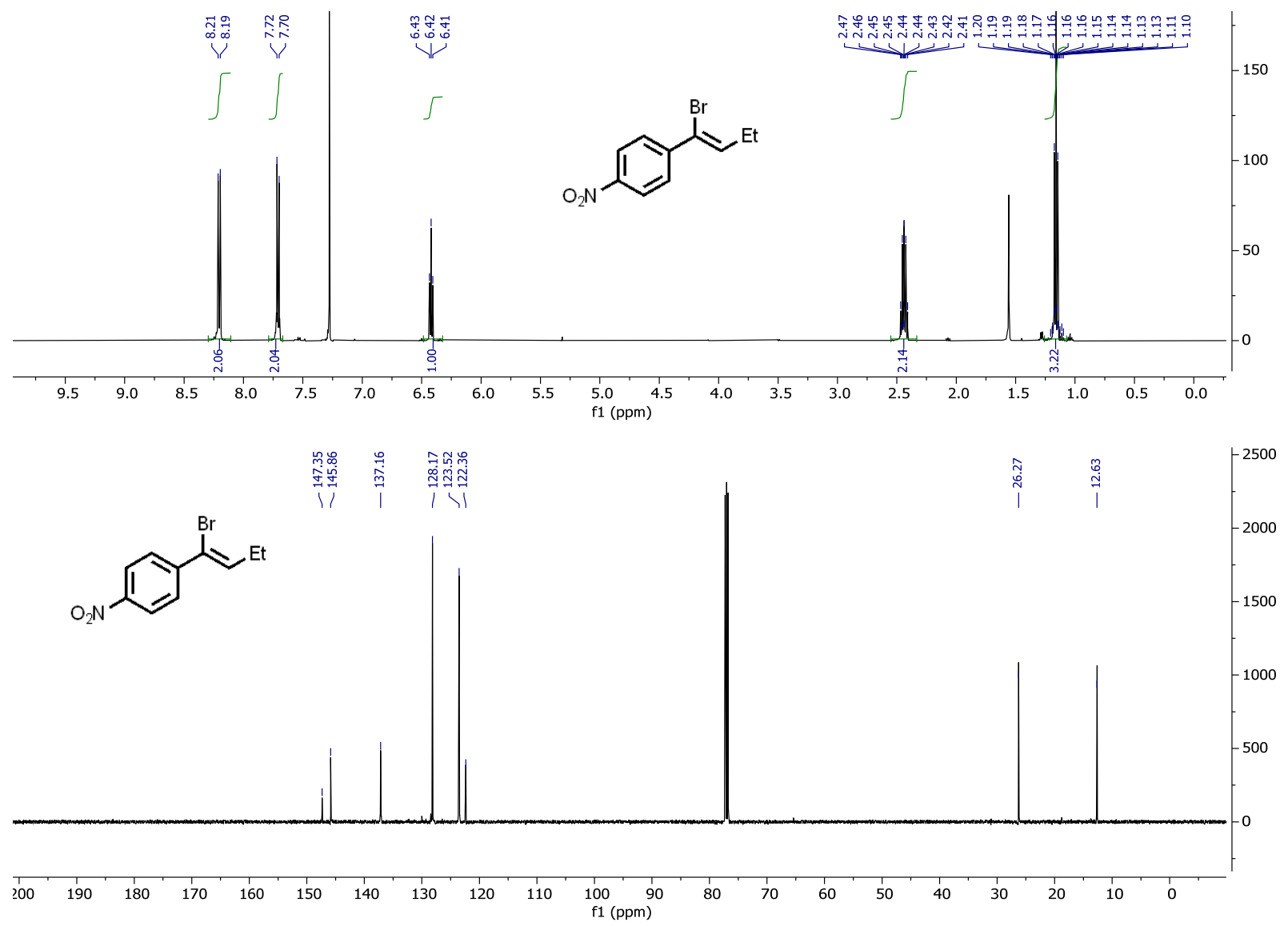
<smiles>CC(C)(C)C=C(Br)c1ccc([N+](=O)[O-])cc1</smiles>

2j, (Z)-1-(1-bromohex-1-en-1-yl)-4-nitrobenzene. Prepared according to General Procedure B from 4nitrophenylboronic acid (167 mg, $1 \mathrm{mmol})$ as an orange oil (140 $\mathrm{mg}, 50 \%$ yield).

${ }^{1} \mathrm{H}$ NMR $\left(500 \mathrm{MHz}, \mathrm{CDCl}_{3}\right) \delta 8.20-8.17(\mathrm{~m}, 2 \mathrm{H}), 7.71-7.67(\mathrm{~m}, 2 \mathrm{H}), 6.41(\mathrm{t}, J=6.9 \mathrm{~Hz}, 1 \mathrm{H}), 2.41$ (q, $J=7.2$ $\mathrm{Hz}, 2 \mathrm{H}), 1.55-1.49(\mathrm{~m}, 2 \mathrm{H}), 1.42(\mathrm{~h}, J=7.2 \mathrm{~Hz}, 2 \mathrm{H}), 0.96(\mathrm{t}, J=7.3 \mathrm{~Hz}, 3 \mathrm{H})$.

${ }^{13} \mathrm{C}$ NMR $\left(126 \mathrm{MHz}, \mathrm{CDCl}_{3}\right) \delta 147.32,145.95,135.94,128.17,123.52,122.78,32.54,30.33,22.43,13.91$.

HRMS (EI): for $\mathrm{C}_{12} \mathrm{H}_{14} \mathrm{BrNO}_{2}$, [M] $]^{+}$calculated $\mathrm{m} / z=283.0202$ and 285.0182 , found $m / z=283.0200$ and 285.0179 .
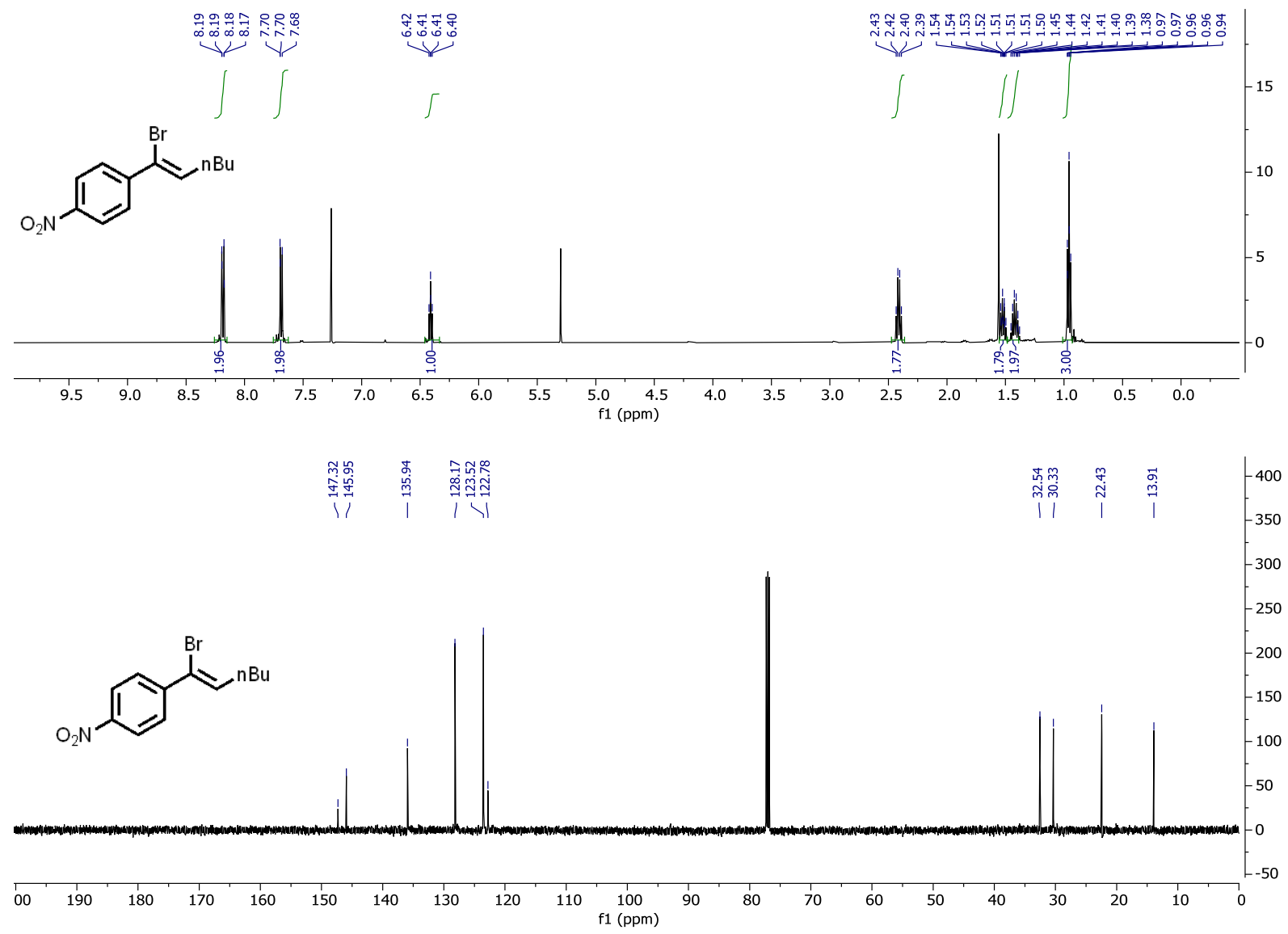
<smiles>C/C=C(\Br)c1ccc(O)cc1</smiles>

2k, (Z)-4-(1-bromoprop-1-en-1-yl)phenyl trifluoromethanesulfonate. Prepared according to General Procedure A from 4-propionylphenyl trifluoromethanesulfonate $(282 \mathrm{mg}, 1 \mathrm{mmol})$ as a clear, colorless oil $(228 \mathrm{mg}, 66 \%)$.

${ }^{1} \mathrm{H} \mathrm{NMR}\left(500 \mathrm{MHz}, \mathrm{CDCl}_{3}\right) \delta 7.61-7.56(\mathrm{~m}, 2 \mathrm{H}), 7.25-7.21(\mathrm{~m}, 2 \mathrm{H}), 6.32(\mathrm{q}, J=6.6 \mathrm{~Hz}, 1 \mathrm{H}), 1.97(\mathrm{~d}, J=6.6$ $\mathrm{Hz}, 3 \mathrm{H})$.

${ }^{13} \mathrm{C}$ NMR $\left(126 \mathrm{MHz}, \mathrm{CDCl}_{3}\right) \delta 149.08,140.38,129.20,128.30,124.26,121.09,118.75$ (q, $\left.J=320.7 \mathrm{~Hz}\right), 18.21$. ${ }^{19} \mathrm{~F}$ NMR $\left(471 \mathrm{MHz}, \mathrm{CDCl}_{3}\right) \delta-72.79$.

HRMS (EI): for $\mathrm{C}_{10} \mathrm{H}_{8} \mathrm{BrF}_{3} \mathrm{O}_{3} \mathrm{~S}$, [M] $]^{+}$calculated $m / z=343.9324$ and 345.9304, found $m / z=343.9219$ and 345.9297 .

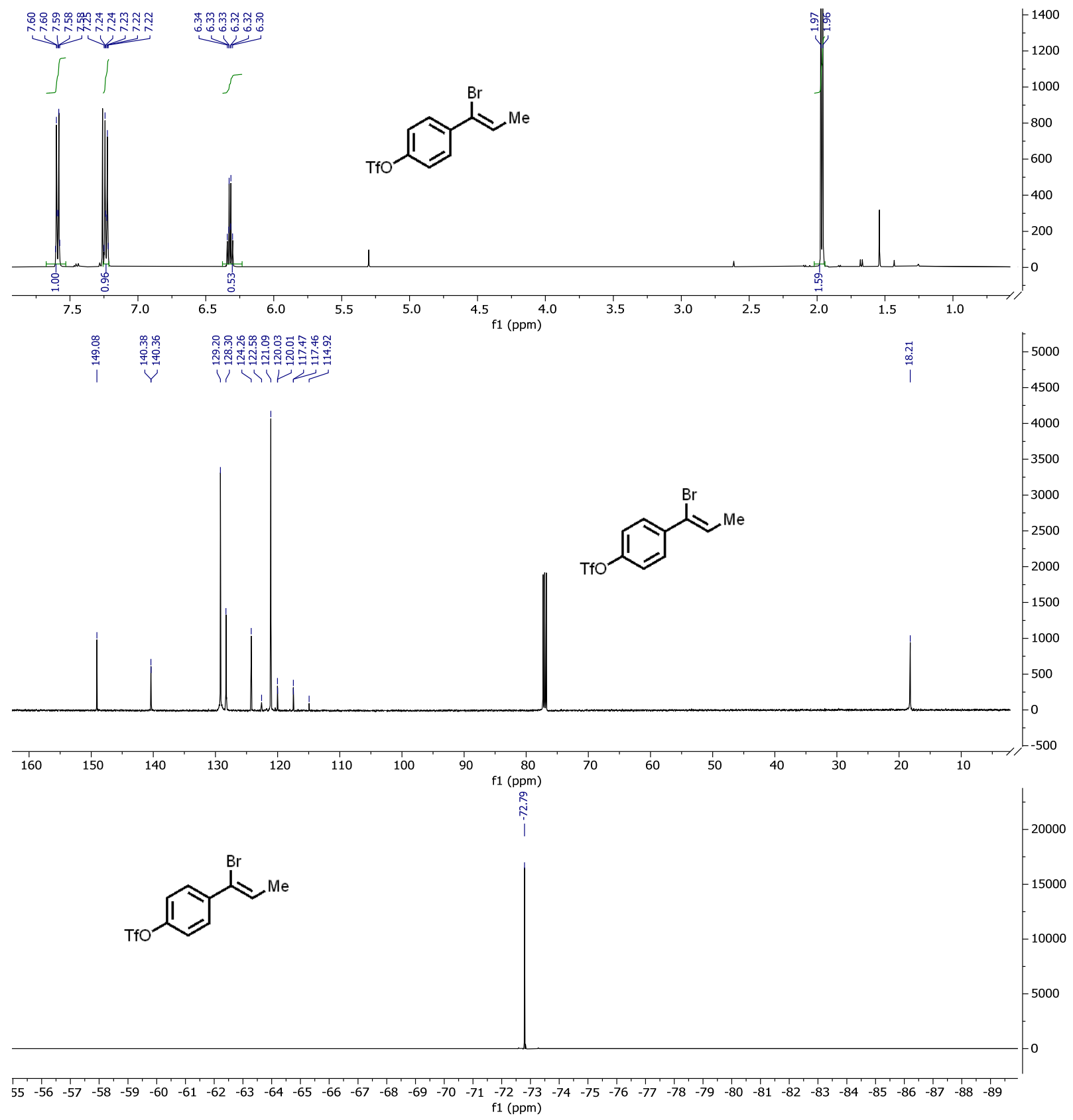




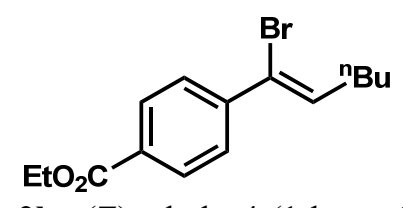

21, (Z)-ethyl 4-(1-bromohex-1-en-1-yl)benzoate. Prepared according to General Procedure B from 4(ethoxycarbonyl)phenylboronic acid (194 mg, $1 \mathrm{mmol})$ as a clear, orange oil (129 mg, 41\% yield).

${ }^{1} \mathrm{H}$ NMR $\left(500 \mathrm{MHz}, \mathrm{CDCl}_{3}\right) \delta 7.99(\mathrm{~d}, J=8.4 \mathrm{~Hz}, 2 \mathrm{H}), 7.59(\mathrm{~d}, J=8.5 \mathrm{~Hz}, 2 \mathrm{H}), 6.33(\mathrm{t}, J=6.9 \mathrm{~Hz}, 1 \mathrm{H}), 4.38(\mathrm{q}, J$ $=7.1 \mathrm{~Hz}, 2 \mathrm{H}), 2.39(\mathrm{q}, J=7.2 \mathrm{~Hz}, 2 \mathrm{H}), 1.55-1.46(\mathrm{~m}, 2 \mathrm{H}), 1.44-1.34(\mathrm{~m}, 5 \mathrm{H}), 0.95(\mathrm{t}, J=7.3 \mathrm{~Hz}, 3 \mathrm{H})$.

${ }^{13} \mathrm{C}$ NMR $\left(126 \mathrm{MHz}, \mathrm{CDCl}_{3}\right) \delta 166.05,144.01,133.88,130.01,129.46,127.33,124.24,61.01,32.39,30.46,22.43$, $14.33,13.94$.

HRMS (ESI): for $\mathrm{C}_{15} \mathrm{H}_{20} \mathrm{BrO}_{2}$, [M+H] $]^{+}$calculated $m / z=311.0642$ and 313.0619, found $m / z=311.0641$ and 313.0621 .
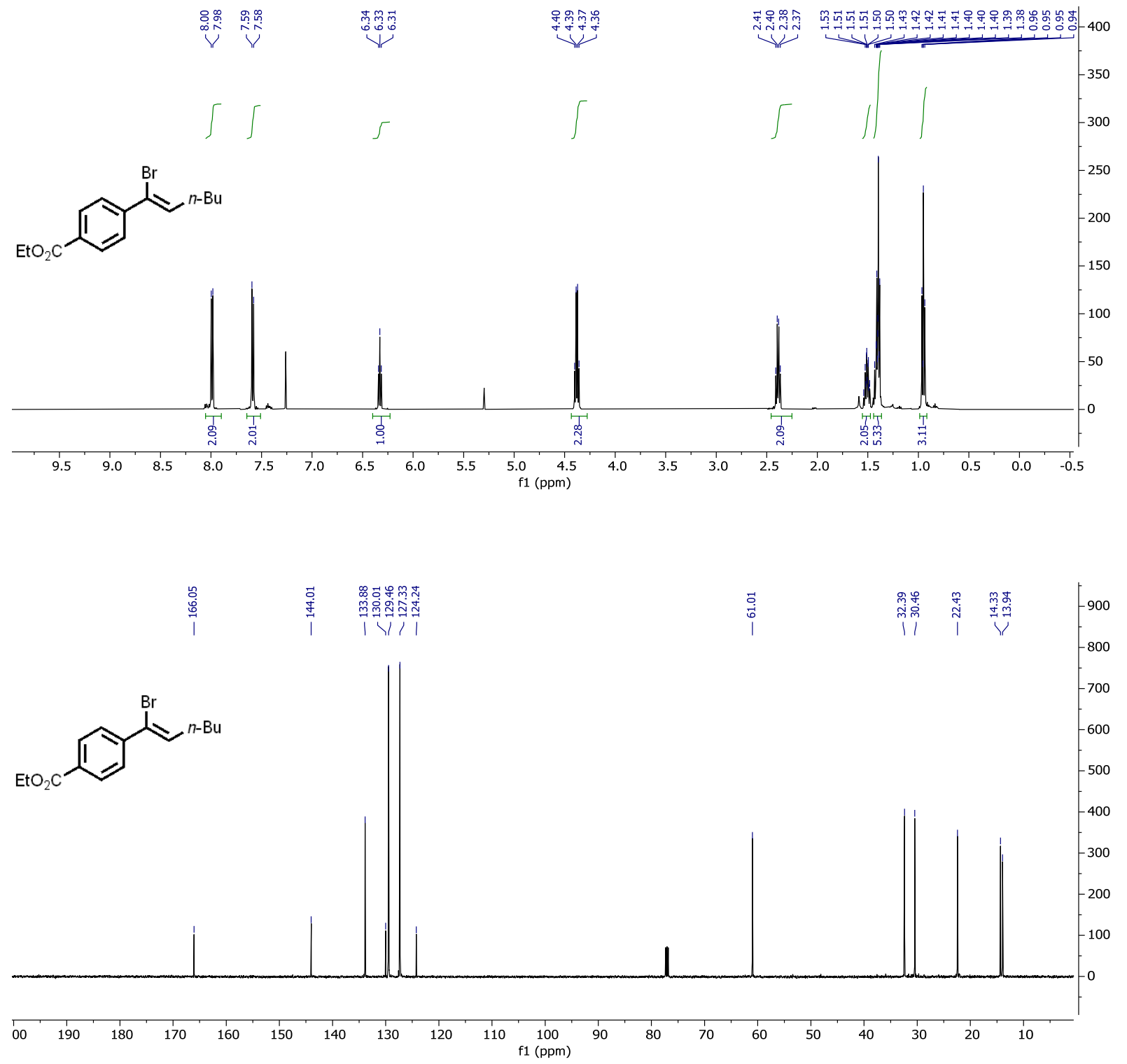
<smiles>C/C=C(\Br)c1ccc(C(F)(F)F)cc1</smiles>

2m, (Z)-1-(1-bromoprop-1-en-1-yl)-4-(trifluoromethyl)benzene. Prepared according to General Procedure A from 4'(trifluoromethyl)propiophenone $(606 \mathrm{mg}, 3 \mathrm{mmol})$ as a clear, colorless oil ( $700 \mathrm{mg}, 88 \%$ yield).

${ }^{1} \mathrm{H}$ NMR $\left(500 \mathrm{MHz}, \mathrm{CDCl}_{3}\right) \delta 7.67-7.62(\mathrm{~m}, 2 \mathrm{H}), 7.62-7.57(\mathrm{~m}, 2 \mathrm{H}), 6.43-6.37(\mathrm{~m}, 1 \mathrm{H}), 2.00(\mathrm{~d}, J=6.6 \mathrm{~Hz}$, $3 \mathrm{H})$.

${ }^{13} \mathrm{C}$ NMR $\left(126 \mathrm{MHz}, \mathrm{CDCl}_{3}\right) \delta 143.35,130.11(\mathrm{q}, J=32.6 \mathrm{~Hz}), 128.50,127.70,125.22(\mathrm{q}, J=3.8 \mathrm{~Hz}), 124.96$, $121.81(\mathrm{~d}, J=271.8 \mathrm{~Hz}), 18.22$.

${ }^{19} \mathrm{~F}$ NMR $\left(471 \mathrm{MHz}, \mathrm{CDCl}_{3}\right) \delta-62.63$.

HRMS (EI): for $\mathrm{C}_{10} \mathrm{H}_{8} \mathrm{BrF}_{3}$, [M] calculated $m / z=263.9756$ and 265.9736, found $m / z=263.9753$ and 265.9732 .
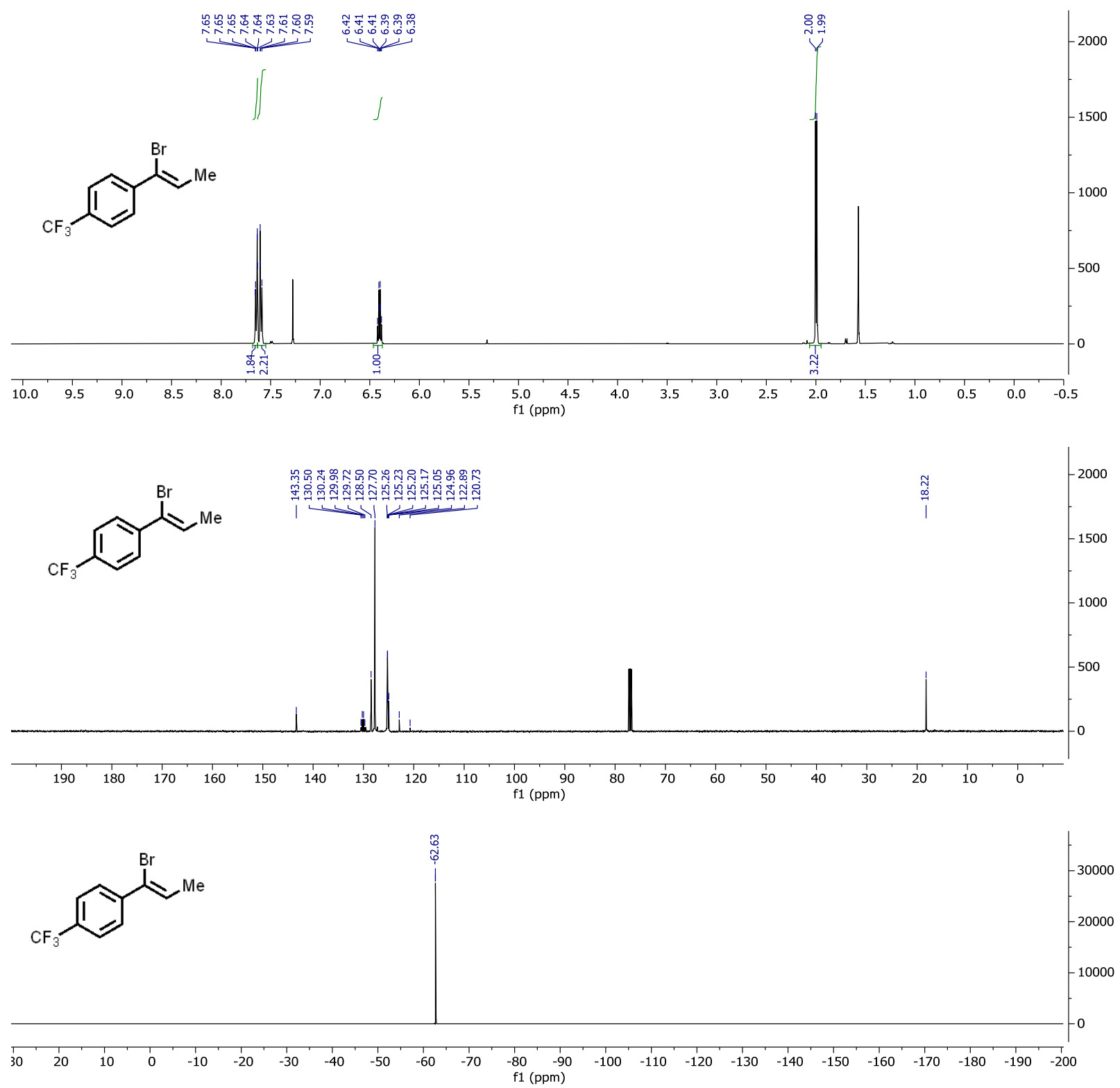
<smiles>C/C=C(\Br)c1ccc(C#N)cc1</smiles>

2n, (Z)-4-(1-bromobut-1-en-1-yl)benzonitrile. Prepared according to General Procedure B from 4cyanophenylboronic acid (441 $\mathrm{mg}, 3 \mathrm{mmol}$ ) as a clear, yellow oil (153 $\mathrm{mg}, 21 \%$ yield).

${ }^{1} \mathrm{H}$ NMR $\left(500 \mathrm{MHz}, \mathrm{CDCl}_{3}\right) \delta 7.66-7.59(\mathrm{~m}, 4 \mathrm{H}), 6.34(\mathrm{t}, J=6.9 \mathrm{~Hz}, 1 \mathrm{H}), 2.45-2.33(\mathrm{~m}, 2 \mathrm{H}), 1.13(\mathrm{t}, J=7.6 \mathrm{~Hz}$, $3 \mathrm{H})$.

${ }^{13} \mathrm{C}$ NMR $\left(126 \mathrm{MHz}, \mathrm{CDCl}_{3}\right) \delta 144.04,136.45,132.05,128.04,122.79,118.52,111.71,26.18,12.66$.

HRMS (ESI): for $\mathrm{C}_{11} \mathrm{H}_{1} \mathrm{BrN}$, $[\mathrm{M}+\mathrm{H}]^{+}$calculated $m / z=236.0069$ and 238.0049, found $m / z=236.0070$ and 238.0049 .
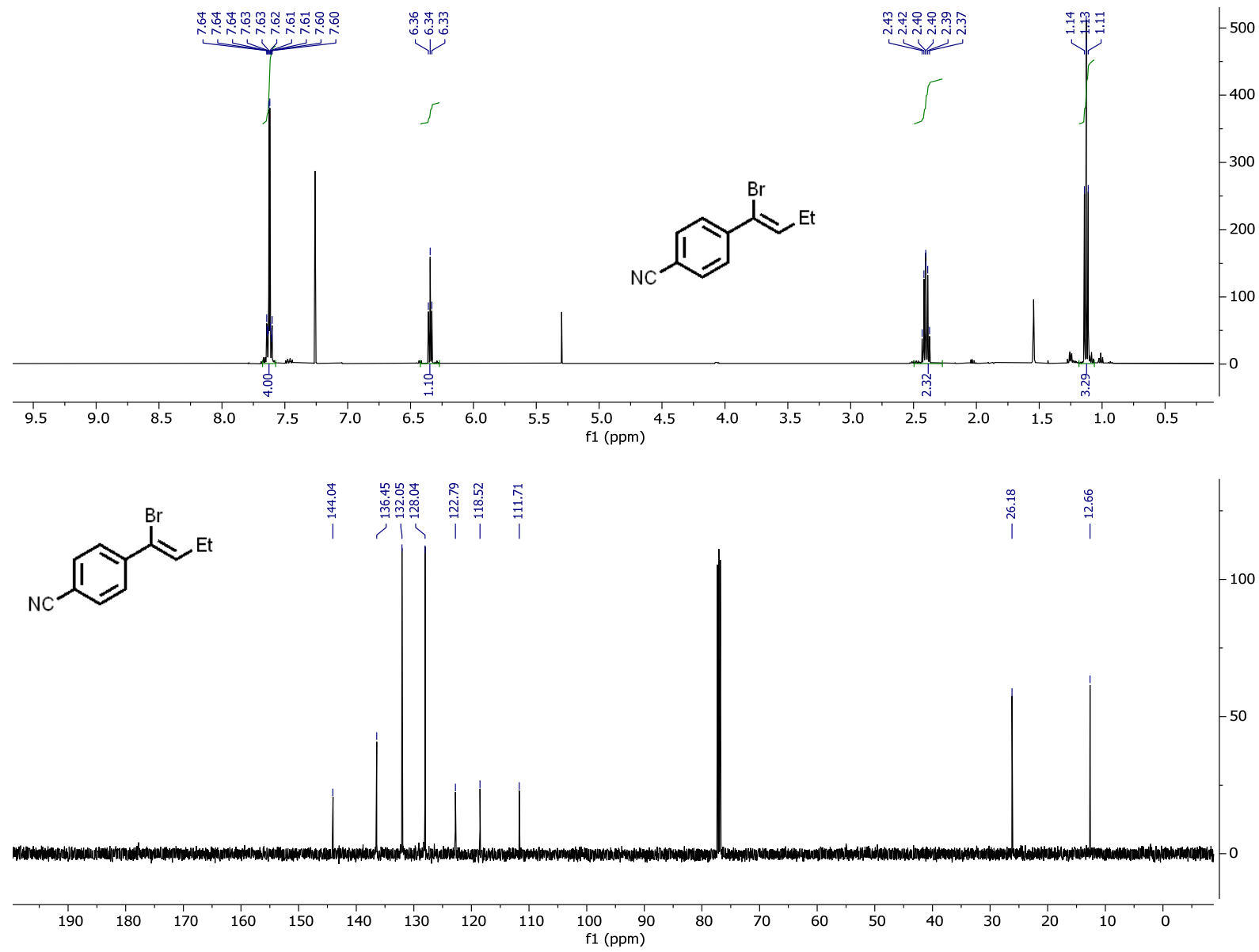
<smiles>CC(C)(C)c1ccc(/C(Br)=C/C#N)cc1</smiles>

2o, (Z)-4-(1-bromohex-1-en-1-yl)benzonitrile. Prepared according to General Procedure B from 4cyanophenylboronic acid (441 mg, $3 \mathrm{mmol}$ ) as a clear, yellow oil (357 $\mathrm{mg}, 45 \%$ yield).

${ }^{1} \mathrm{H} \mathrm{NMR}\left(600 \mathrm{MHz}, \mathrm{CDCl}_{3}\right) \delta 7.64-7.59(\mathrm{~m}, 4 \mathrm{H}), 6.35(\mathrm{t}, J=6.9 \mathrm{~Hz}, 1 \mathrm{H}), 2.39$ (q, $\left.J=7.2 \mathrm{~Hz}, 2 \mathrm{H}\right), 1.54-1.48$ (m, 2H), $1.44-1.36(\mathrm{~m}, 2 \mathrm{H}), 0.95(\mathrm{t}, J=7.3 \mathrm{~Hz}, 3 \mathrm{H})$.

${ }^{13} \mathrm{C}$ NMR $\left(126 \mathrm{MHz}, \mathrm{CDCl}_{3}\right) \delta 144.11,135.21,132.04,128.04,123.22,118.52,111.68,32.44,30.35,22.41,13.91$. HRMS (ESI): for $\mathrm{C}_{13} \mathrm{H}_{15} \mathrm{BrN}$, [M+H] $]^{+}$calculated $\mathrm{m} / z=264.0382$ and 266.0362, found $\mathrm{m} / z=264.0382$ and 266.0361 .
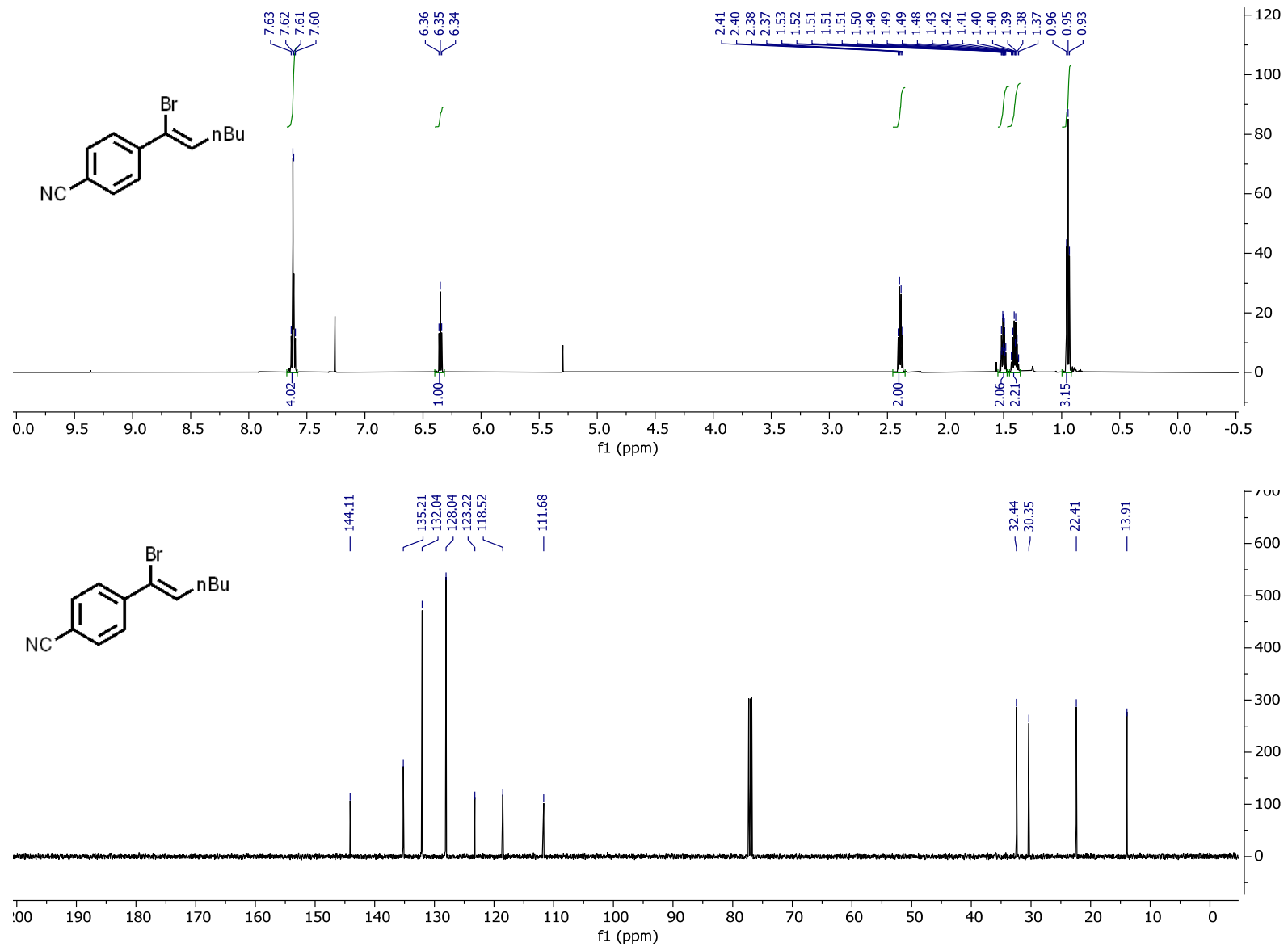
<smiles>C/C=C(\Br)c1ccc(Br)cc1</smiles>

2p, (Z)-1-bromo-4-(1-bromoprop-1-en-1-yl)benzene. Prepared according to General Procedure A from 3'bromopropiophenone (213 mg, $1 \mathrm{mmol}$ ) as a clear, colorless oil (160 mg, 57\% yield).

${ }^{1} \mathrm{H}$ NMR $\left(500 \mathrm{MHz}, \mathrm{CDCl}_{3}\right) \delta 7.46(\mathrm{~d}, J=9.1 \mathrm{~Hz}, 2 \mathrm{H}), 7.40(\mathrm{~d}, J=7.9 \mathrm{~Hz}, 2 \mathrm{H}), 6.30(\mathrm{qd}, J=6.5,0.9 \mathrm{~Hz}, 1 \mathrm{H}), 1.95$ $(\mathrm{d}, J=6.6 \mathrm{~Hz}, 3 \mathrm{H})$.

${ }^{13} \mathrm{C}$ NMR $\left(126 \mathrm{MHz}, \mathrm{CDCl}_{3}\right) \delta 139.02,131.32,128.99,126.98,125.35,122.27,18.22$.

HRMS (EI): for $\mathrm{C}_{9} \mathrm{H}_{8} \mathrm{Br}_{2},[\mathrm{M}]^{+}$calculated $\mathrm{m} / z=273.8987$ and 275.8967 and 277.8946 , found $\mathrm{m} / z=273.8986$ and 275.8962 and 277.8943.

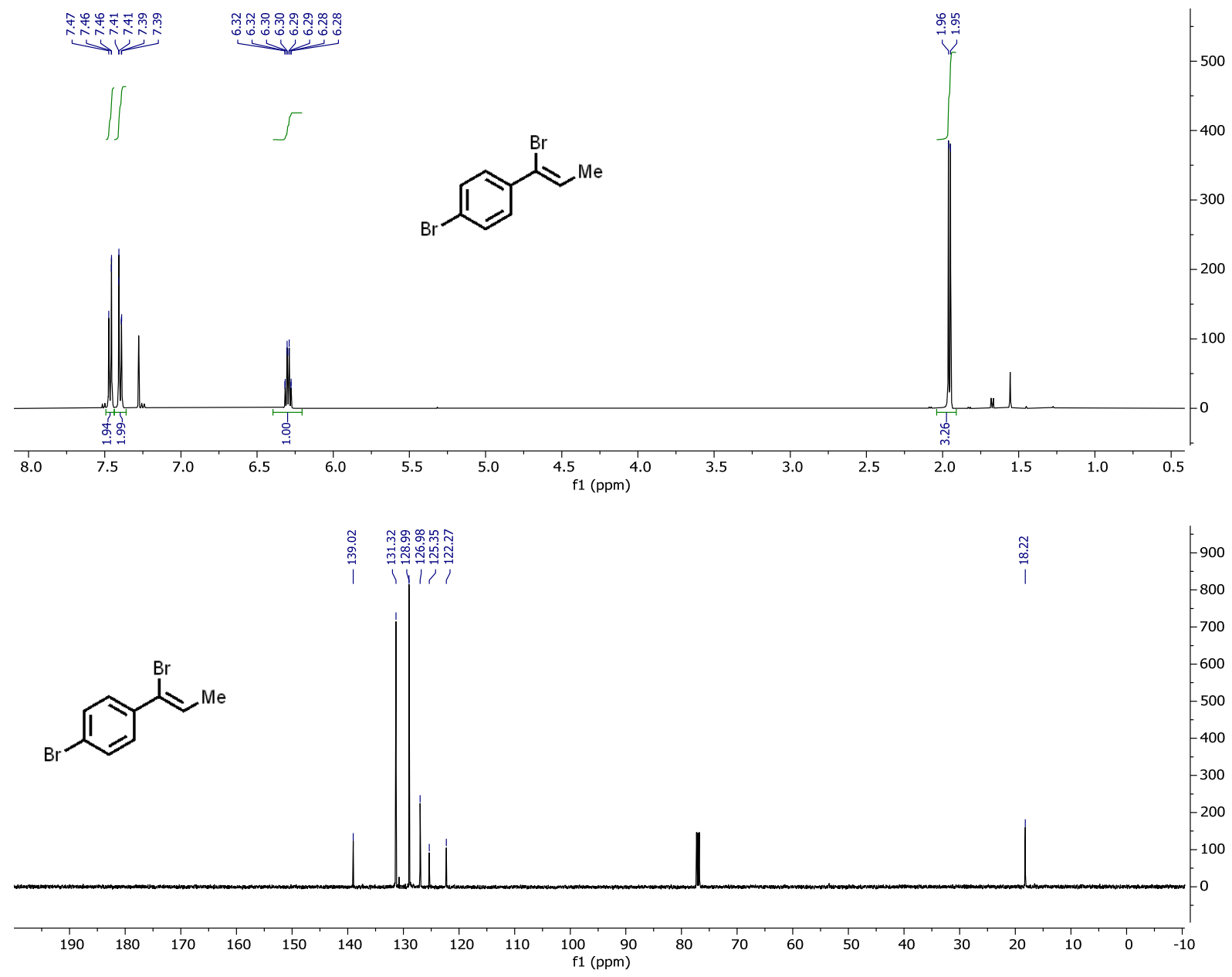


<smiles>C/C=C(\Br)c1cccc(Cl)c1</smiles>

2q, (Z)-1-(1-bromoprop-1-en-1-yl)-3-chlorobenzene. Prepared according to General Procedure A from 3'chloropropiophenone (169 mg, $1 \mathrm{mmol}$ ) as a clear, colorless oil (209 $\mathrm{mg}, 85 \%)$.

${ }^{1} \mathrm{H}$ NMR $\left(500 \mathrm{MHz}, \mathrm{CDCl}_{3}\right) \delta 7.54-7.50(\mathrm{~m}, 1 \mathrm{H}), 7.44-7.38(\mathrm{~m}, 1 \mathrm{H}), 7.30-7.23(\mathrm{~m}, 2 \mathrm{H}), 6.33(\mathrm{q}, J=6.7 \mathrm{~Hz}$, $1 \mathrm{H}), 1.97(\mathrm{~d}, J=6.6 \mathrm{~Hz}, 3 \mathrm{H})$.

${ }^{13} \mathrm{C}$ NMR (126 MHz, $\left.\mathrm{CDCl}_{3}\right) \delta 142.02,134.40,129.66,128.15,125.87,125.14,18.44$.

HRMS (EI): for $\mathrm{C}_{9} \mathrm{H}_{8} \mathrm{BrCl},[\mathrm{M}]^{+}$calculated $m / z=229.9492$ and 231.9472 , found $m / z=229.9492$ and 231.9468 .
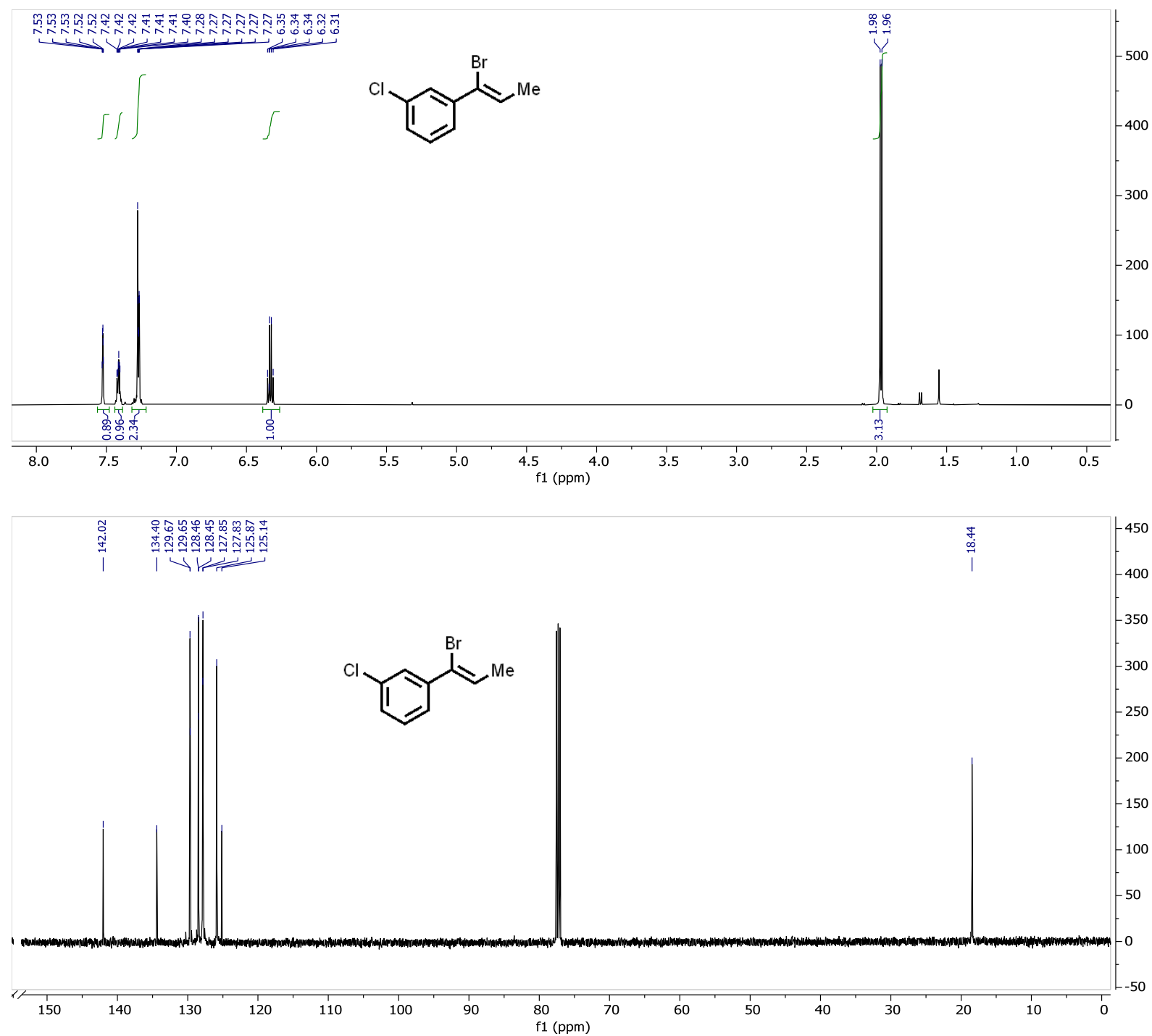
<smiles>C/C=C(\Br)c1cnn(C)c1</smiles>

2r, (Z)-4-(1-bromoprop-1-en-1-yl)-1-methyl-1H-pyrazole. Prepared according to General Procedure A from 1-(1methyl-1H-pyrazol-4-yl)propan-1-one (138 mg, $1 \mathrm{mmol}$ ) as a clear, colorless oil (45 $\mathrm{mg}, 23 \%$ yield).

${ }^{1} \mathrm{H}$ NMR $\left(600 \mathrm{MHz}, \mathrm{CDCl}_{3}\right) \delta 7.56(\mathrm{~s}, 1 \mathrm{H}), 7.44(\mathrm{~s}, 1 \mathrm{H}), 6.16(\mathrm{q}, J=6.6 \mathrm{~Hz}, 1 \mathrm{H}), 3.86(\mathrm{~s}, 3 \mathrm{H}), 1.87(\mathrm{~d}, J=6.6 \mathrm{~Hz}$, $3 \mathrm{H})$.

${ }^{13} \mathrm{C}$ NMR $\left(126 \mathrm{MHz}, \mathrm{CDCl}_{3}\right) \delta 136.28,129.13,123.71,122.19,116.05,39.03,17.19$.

HRMS (ESI): for $\mathrm{C}_{7} \mathrm{H}_{10} \mathrm{BrN}_{2},[\mathrm{M}+\mathrm{H}]^{+}$calculated $\mathrm{m} / z=201.0022$ and 203.0001, found $\mathrm{m} / z=201.0022$ and 202.9999 .

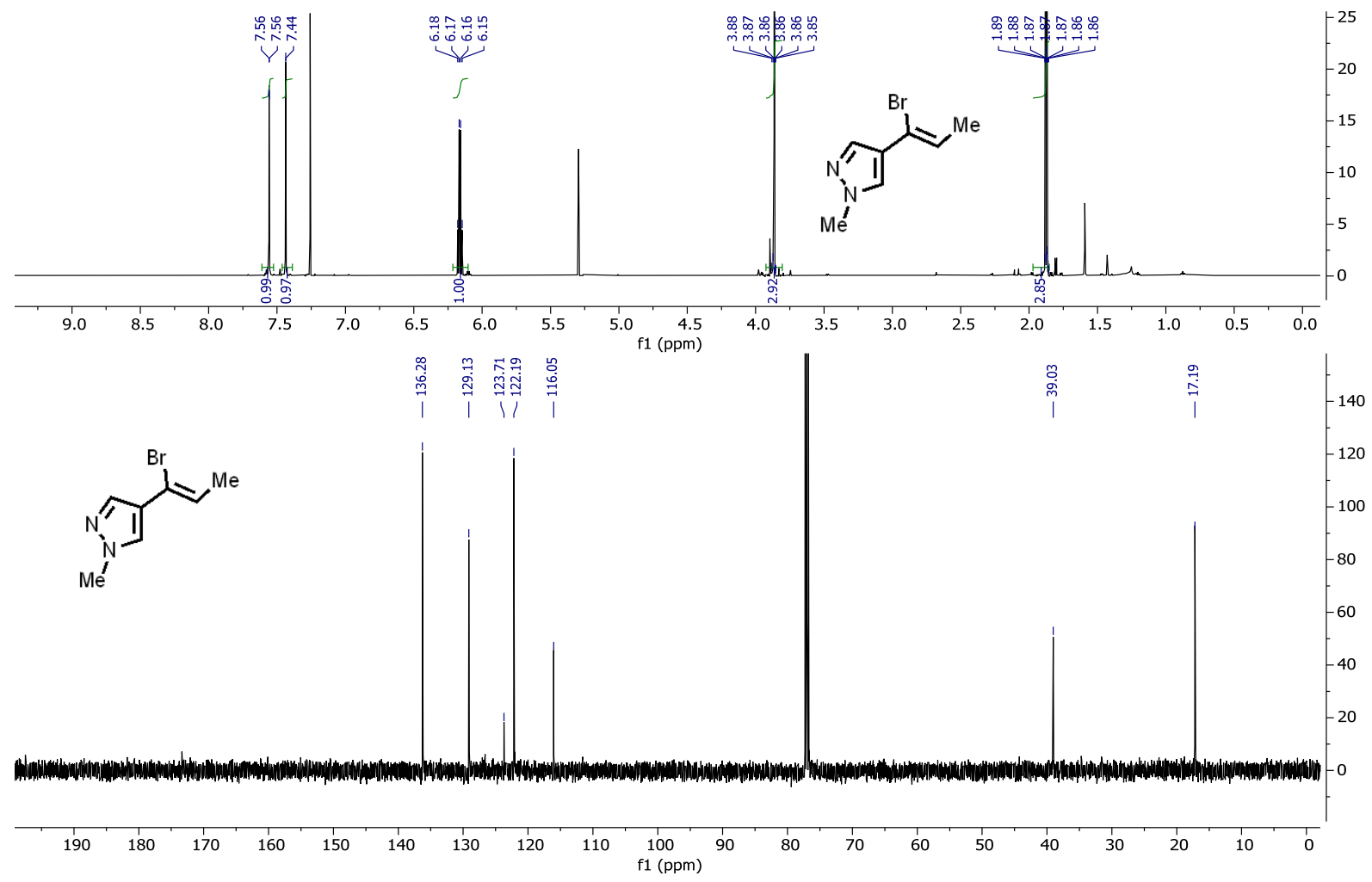




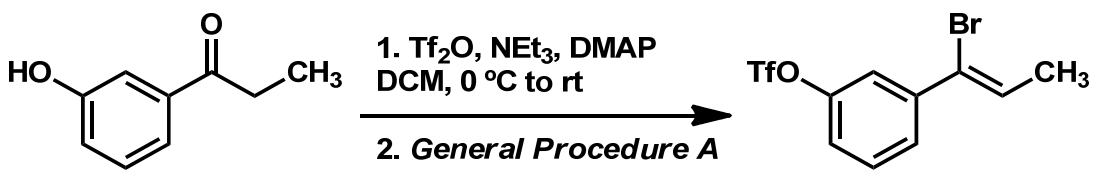

To a solution of 3'-hydroxypropiophene (750 mg, $5 \mathrm{mmol}, 1$ equiv) in DCM (10 mL, 0.5M) was added NEt 3 (2.1 $\mathrm{mL}$, $15 \mathrm{mmol}, 3$ equiv) and DMAP $\left(61 \mathrm{mg}, 0.5 \mathrm{mmol}, 0.1\right.$ equiv). The solution was cooled to $0{ }^{\circ} \mathrm{C}$ and $\mathrm{Tf}_{2} \mathrm{O}(5.5 \mathrm{~mL}, 1 \mathrm{M}$ in DCM, 1.1 equiv) was added dropwise via syringe. The solution was stirred at $0{ }^{\circ} \mathrm{C}$ for 30 minutes and was then warmed to room temperature and stirred for an additional 3 hours. After this time, $1 \mathrm{M} \mathrm{HCl}$ was added carefully, and the solution was diluted with $\mathrm{Et}_{2} \mathrm{O}$. The layers were separated, and the organic layer washed with $1 \mathrm{M} \mathrm{HCl}(2 \mathrm{x})$, water, $\mathrm{NaHCO}_{3}$ (sat. aqueous), and brine. The organic layer was then dried over $\mathrm{Na}_{2} \mathrm{SO}_{4}$, concentrated in vacuo, and purified by column chromatography to afford 3-propionylphenyl trifluoromethanesulfonate (S1) as a clear, colorless oil (1.02 g, $72 \%$ yield).

${ }^{1} \mathrm{H}$ NMR $\left(500 \mathrm{MHz}, \mathrm{CDCl}_{3}\right) \delta 7.98(\mathrm{ddd}, J=7.6,1.5,0.9 \mathrm{~Hz}, 1 \mathrm{H}), 7.86(\mathrm{dd}, J=2.6,1.5 \mathrm{~Hz}, 1 \mathrm{H}), 7.58(\mathrm{t}, J=8.0$ $\mathrm{Hz}, 1 \mathrm{H}), 7.47$ (ddd, $J=8.2,2.5,1.0 \mathrm{~Hz}, 1 \mathrm{H}), 3.01(\mathrm{q}, J=7.2 \mathrm{~Hz}, 2 \mathrm{H}), 1.25$ (t, $J=7.2 \mathrm{~Hz}, 2 \mathrm{H})$.

${ }^{13} \mathrm{C}$ NMR $\left(126 \mathrm{MHz}, \mathrm{CDCl}_{3}\right) \delta 198.45,149.80,139.10,130.61,127.76,125.47,120.73,118.69$ (q, $\left.J=320.8 \mathrm{~Hz}\right)$, $32.02,7.88$.

${ }^{19} \mathrm{~F}$ NMR $\left(471 \mathrm{MHz}, \mathrm{CDCl}_{3}\right) \delta-72.80$.

HRMS (ESI): for $\mathrm{C}_{10} \mathrm{H}_{10} \mathrm{~F}_{3} \mathrm{O}_{4} \mathrm{~S}$, $[\mathrm{M}+\mathrm{H}]^{+}$calculated $m / z=283.0246$, found $m / z=283.0246$.
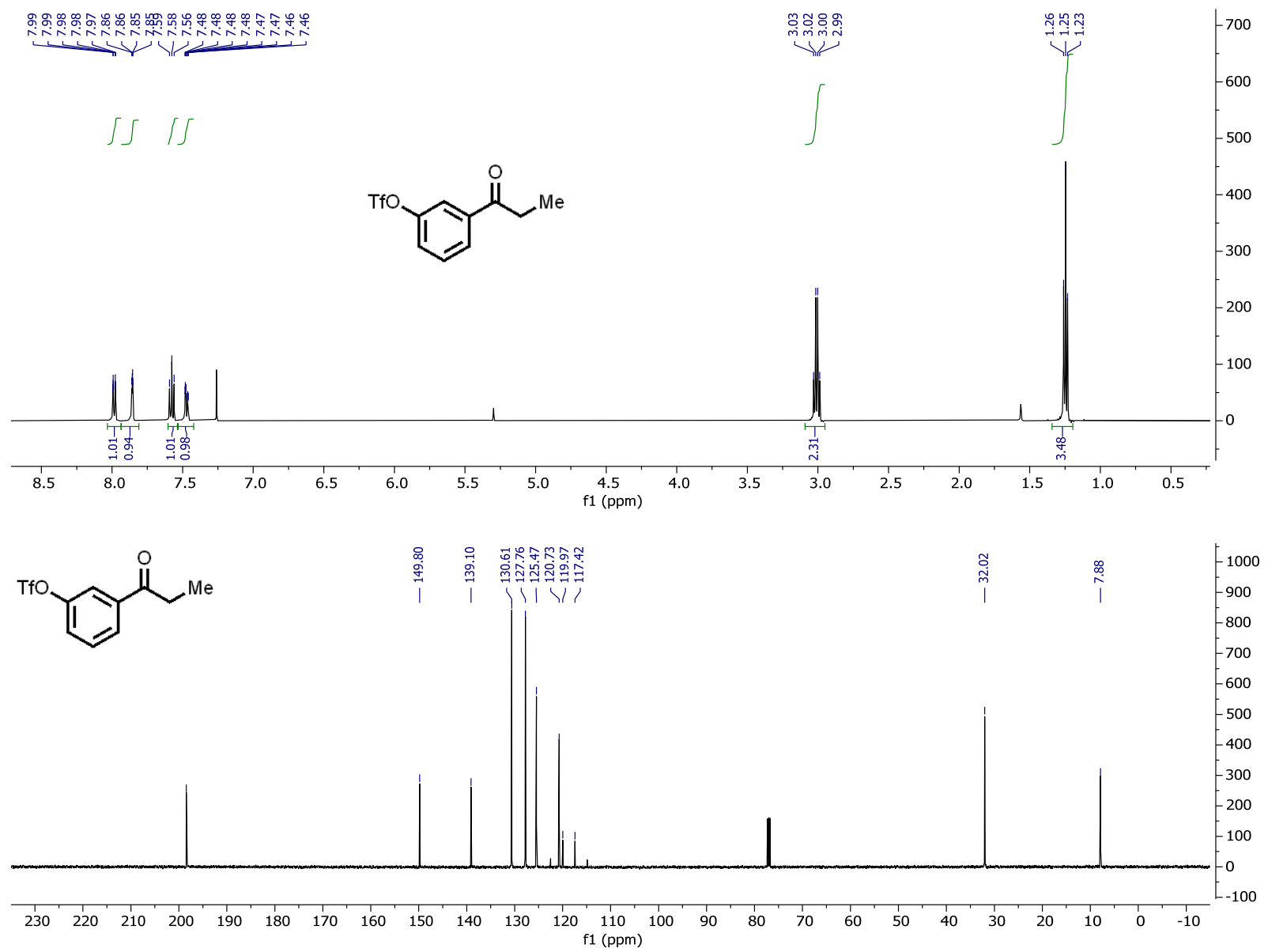


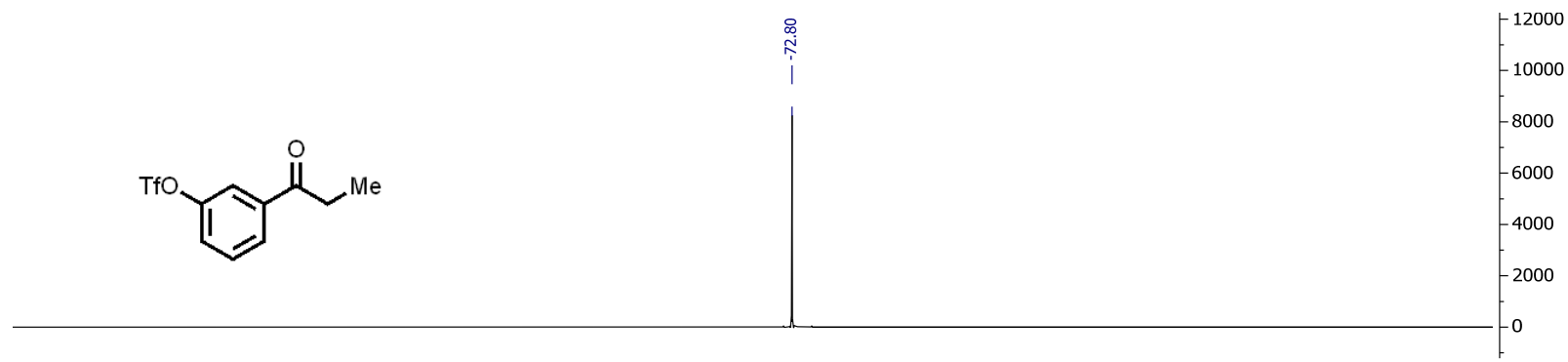

$\begin{array}{lllllllllllllllllllllllllllllllllllllll}1 & -55 & -56 & -57 & -58 & -59 & -60 & -61 & -62 & -63 & -64 & -65 & -66 & -67 & -68 & -69 & -70 & -71 & -72 & -73 & -74 & -75 & -76 & -77 & -78 & -79 & -80 & -81 & -82 & -83 & -84 & -85 & -86 & -87 & -88 & -89\end{array}$

2b, (Z)-3-(1-bromoprop-1-en-1-yl)phenyl trifluoromethanesulfonate was then prepared according to General Procedure A from S1 (282 mg, $1 \mathrm{mmol}$ ) as a clear, colorless oil (214 mg, 62\%).

${ }^{1} \mathrm{H}$ NMR $\left(500 \mathrm{MHz}, \mathrm{CDCl}_{3}\right) \delta 7.57-7.53(\mathrm{~m}, 1 \mathrm{H}), 7.44-7.38(\mathrm{~m}, 2 \mathrm{H}), 7.19(\mathrm{dd}, J=8.3,2.5 \mathrm{~Hz}, 1 \mathrm{H}), 6.37(\mathrm{q}, J=$ $6.6 \mathrm{~Hz}, 1 \mathrm{H}), 1.97(\mathrm{~d}, J=6.6 \mathrm{~Hz}, 3 \mathrm{H})$.

${ }^{13} \mathrm{C}$ NMR $\left(126 \mathrm{MHz}, \mathrm{CDCl}_{3}\right) \delta 149.35,142.57,129.94,128.76,127.21,123.95,120.76,120.44,118.73(\mathrm{~d}, J=320.7$ $\mathrm{Hz}), 18.22$.

${ }^{19} \mathrm{~F}$ NMR $\left(471 \mathrm{MHz}, \mathrm{CDCl}_{3}\right) \delta-72.83$.

HRMS (EI): for $\mathrm{C}_{10} \mathrm{H}_{8} \mathrm{BrF}_{3} \mathrm{O}_{3} \mathrm{~S}$, [M] $]^{+}$calculated $m / z=343.9324$ and 345.9304 , found $m / z=343.9325$ and 345.9302 .
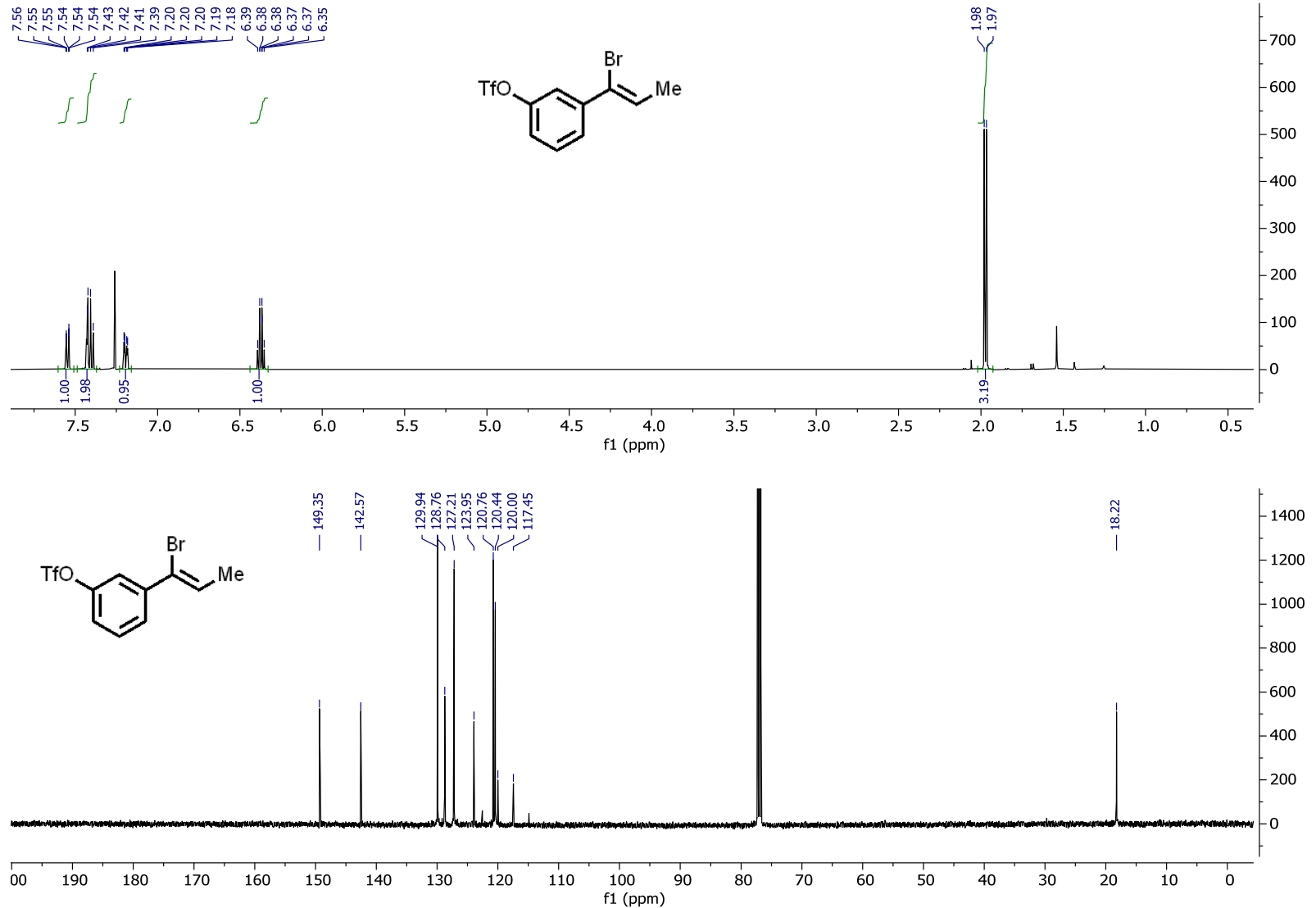

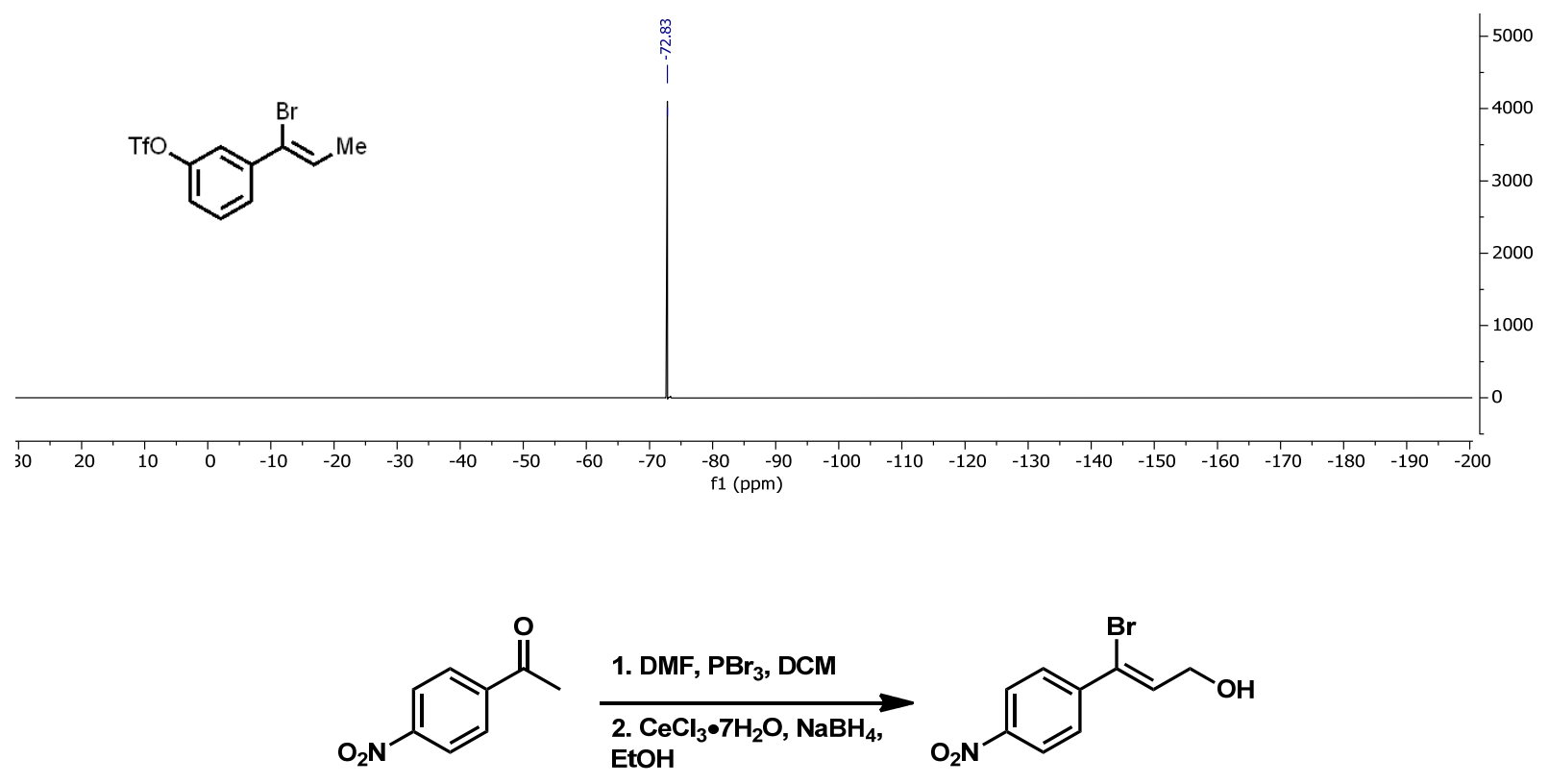

(Z)-3-bromo-3-(4-nitrophenyl)prop-2-en-1-ol. 2s was prepared according to a modified procedure by Shunatona, et. al. ${ }^{3} \mathrm{~N}, N$-Dimethylformamide $\left(7.7 \mathrm{~mL}, 100 \mathrm{mmol}, 5\right.$ equiv) was cooled to $0{ }^{\circ} \mathrm{C}$ in dichloromethane $(100 \mathrm{~mL}, 0.2 \mathrm{M})$. Phosphorus tribromide $\left(8.2 \mathrm{~mL}, 86 \mathrm{mmol}, 4.3\right.$ equiv) was added dropwise and the mixture was stirred at $0{ }^{\circ} \mathrm{C}$ for $2 \mathrm{~h}$. 4-nitroacetophenone $(3.3 \mathrm{~g}, 20 \mathrm{mmol})$ was dissolved in dichloromethane and was added via canula to the stirring mixture. The reaction mixture was heated at reflux at $40{ }^{\circ} \mathrm{C}$ for $12 \mathrm{~h}$. The reaction mixture was poured over cold $\mathrm{NaHCO}_{3}$, extracted with $\mathrm{Et}_{2} \mathrm{O}$, and dried over $\mathrm{Na}_{2} \mathrm{SO}_{4}$. The solvent was removed in vacuo to give aldehyde, which was taken directly to the next step.

A portion of the crude aldehyde was dissolved in $\mathrm{EtOH}(10 \mathrm{~mL})$ at $0{ }^{\circ} \mathrm{C}$. Cerium(III) chloride heptahydrate $(1.3 \mathrm{~g}, 1.2$ equiv) was then added all at once. Sodium borohydride ( $0.441 \mathrm{~g}, 12 \mathrm{mmol}, 4$ equiv) was carefully added. The reaction mixture was warmed to ambient temperature and stirred for $30 \mathrm{~min}$. Upon completion the mixture was quenched with acetone and was stirred for $1 \mathrm{~h}$. Saturated $\mathrm{NH}_{4} \mathrm{Cl}$ was added to the reaction, and the solvent was removed under vacuum. The mixture was extracted with $\mathrm{Et}_{2} \mathrm{O}$, dried over $\mathrm{Na}_{2} \mathrm{SO}_{4}$, and the solvent was removed in vacuo. The crude product was filtered through a short pad of silica to give alcohol $2 \mathbf{s}(686 \mathrm{mg}, 91 \%)$ as a white solid.

${ }^{1} \mathrm{H}$ NMR $\left(500 \mathrm{MHz}, \mathrm{CDCl}_{3}\right) \delta 8.23(\mathrm{dt}, J=9.4,1.7 \mathrm{~Hz}, 2 \mathrm{H}), 7.74(\mathrm{dt}, J=7.8,0.9 \mathrm{~Hz}, 2 \mathrm{H}), 6.67(\mathrm{td}, J=5.6,1.1 \mathrm{~Hz}$, $1 \mathrm{H}), 4.53(\mathrm{t}, J=5.3 \mathrm{~Hz}, 2 \mathrm{H}), 1.85-1.71(\mathrm{~m}, 1 \mathrm{H})$.

${ }^{13} \mathrm{C}$ NMR $\left(126 \mathrm{MHz}, \mathrm{CDCl}_{3}\right) \delta 147.79,144.75,134.15,128.24,123.66,122.89,63.38$.

HRMS (ESI): for $\mathrm{C}_{9} \mathrm{H}_{7} \mathrm{BrNO}_{3}$, [M-H] ${ }^{+}$calculated $\mathrm{m} / z=255.9615$ and 257.9594 , found $\mathrm{m} / z=255.9616$ and 257.9595 . 

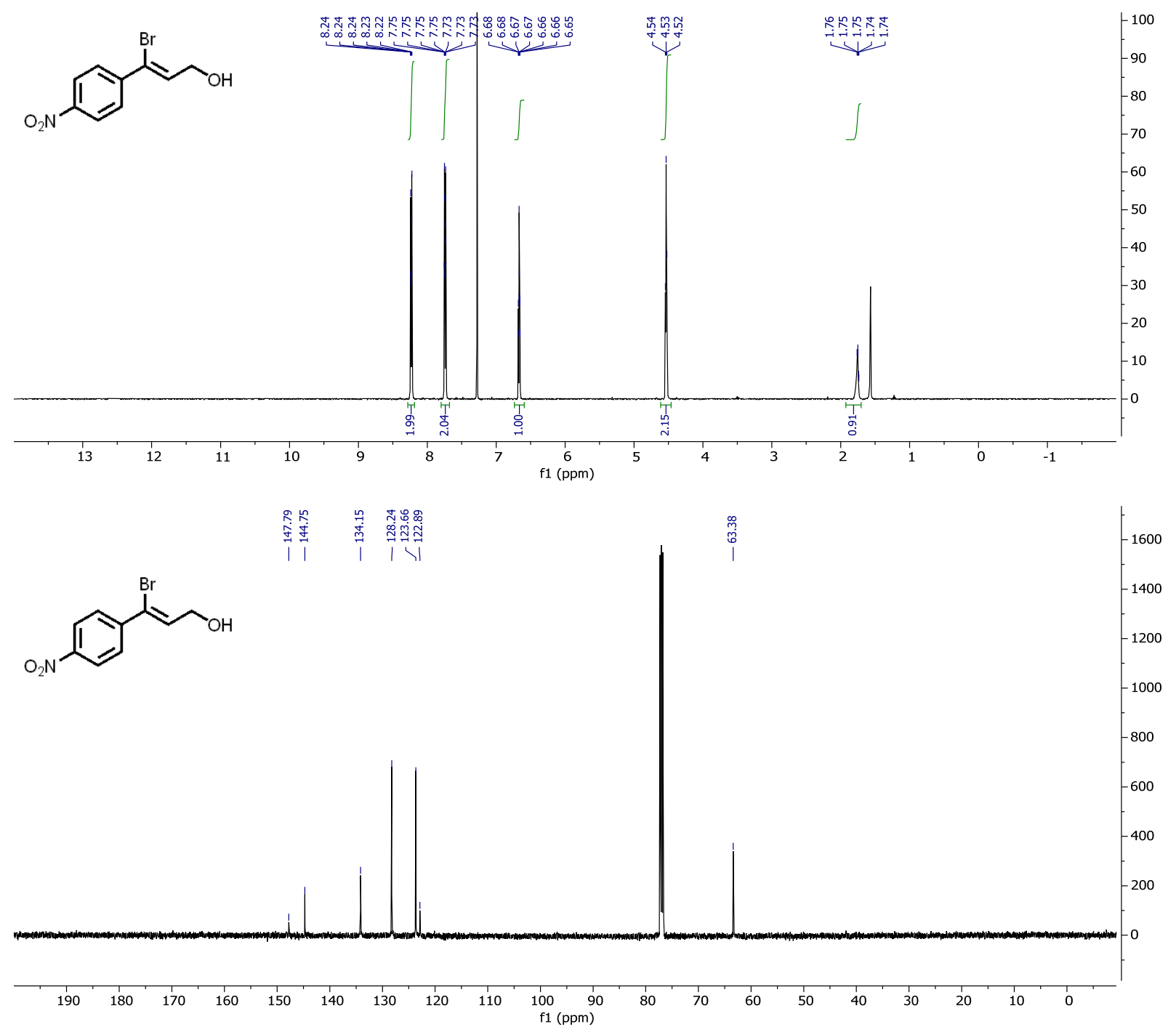
<smiles>O=C(Cl)c1cccc(Br)c1</smiles>

(Z)-1-bromo-3-(1,4-dibromobut-1-en-1-yl)benzene. $2 \mathbf{t}$ was prepared according to a modified procedure by Murphy and coworker. ${ }^{4}$ To a flame-dried round-bottom flask equipped with a stir bar was added THF $(40 \mathrm{~mL}, 0.1 \mathrm{M})$ and NaHMDS (10.5 mL, $1 \mathrm{M}$ in THF, 2.1 equiv). The flask was then cooled to $-78{ }^{\circ} \mathrm{C}$ and $\gamma$-butyrolactone $(0.38 \mathrm{~mL}, 5$ mmol, 1 equiv) was added dropwise via syringe. The reaction mixture was then stirred for $1 \mathrm{~h}$. After this time, 3bromobenzoylchloride $(0.66 \mathrm{~mL}, 5 \mathrm{mmol}, 1$ equiv) was added via syringe, and the solution was stirred for 30 minutes. After this time, the reaction was quenched with $1 \mathrm{M} \mathrm{HCl}$ and diluted with EtOAc. The organic layers were then washed with water and brine, dried over $\mathrm{Na}_{2} \mathrm{SO}_{4}$, and concentrated in vacuo to give the $\beta$-ketoester, which was used without further purification.

The crude $\beta$-ketoester was then dissolved in 1:1:1 THF:MeOH: $\mathrm{H}_{2} \mathrm{O}(8 \mathrm{~mL}$ each $)$ in a vial equipped with a stir bar and septum cap. Conc. $\mathrm{HCl}(\sim 0.1 \mathrm{~mL})$ was then added, and the vial sealed and heated to $65^{\circ} \mathrm{C}$. Upon completion by TLC, the mixture was cooled to room temperature, diluted with EtOAc, and washed with water and brine. The organic layer was then dried over $\mathrm{Na}_{2} \mathrm{SO}_{4}$ and concentrated in vacuo. The crude $\gamma$-hydroxyketone was then used without further purification.

The vinyl bromide was then synthesized according to General Procedure A without the DBU elimination as a clear, colorless oil (387 mg, 21\% yield over 3 steps).

${ }^{1} \mathrm{H}$ NMR $\left(500 \mathrm{MHz}, \mathrm{CDCl}_{3}\right) \delta 7.68$ (q, $\left.J=1.9,1.4 \mathrm{~Hz}, 1 \mathrm{H}\right), 7.46$ (two overlapping dt, $J=12.5,8.0,2.1,1.0 \mathrm{~Hz}$, $2 \mathrm{H}), 7.22(\mathrm{td}, J=7.9,1.0 \mathrm{~Hz}, 1 \mathrm{H}), 6.31(\mathrm{td}, J=6.7,1.0 \mathrm{~Hz}, 1 \mathrm{H}), 3.52(\mathrm{td}, J=6.8,1.0 \mathrm{~Hz}, 2 \mathrm{H}), 2.95(\mathrm{qd}, J=6.7,1.0$ $\mathrm{Hz}, 2 \mathrm{H})$.

${ }^{13} \mathrm{C}$ NMR $\left(126 \mathrm{MHz}, \mathrm{CDCl}_{3}\right) \delta 141.40,131.70,130.54,129.79,129.37,126.25,125.99,122.36,35.52,30.65$. HRMS (EI): for $\mathrm{C}_{10} \mathrm{H}_{9} \mathrm{Br}_{3},[\mathrm{M}]^{+}$calculated $\mathrm{m} / \mathrm{z}=365.8249$ and 367.8228 and 369.8208 and 371.8187 , found $\mathrm{m} / \mathrm{z}=$ 365.8247 and 367.8225 and 369.8204 and 371.8184 .

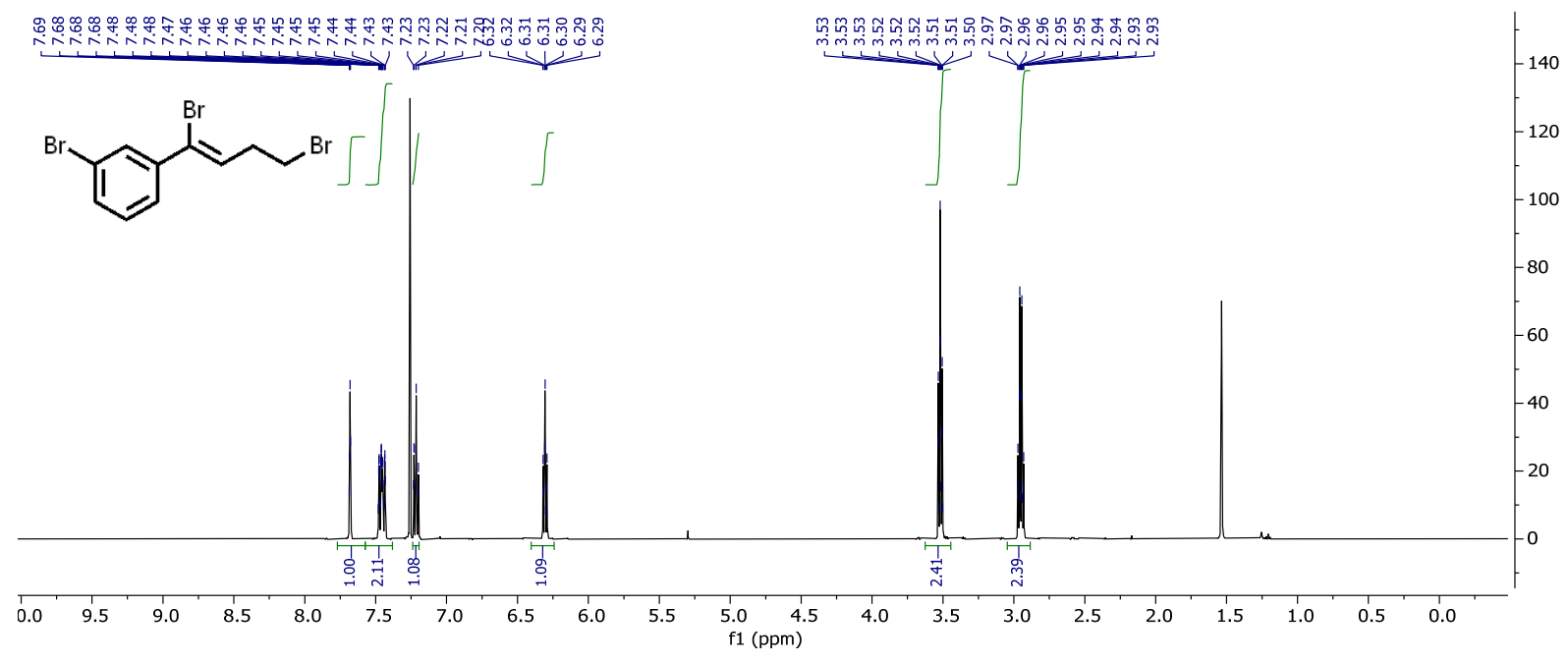




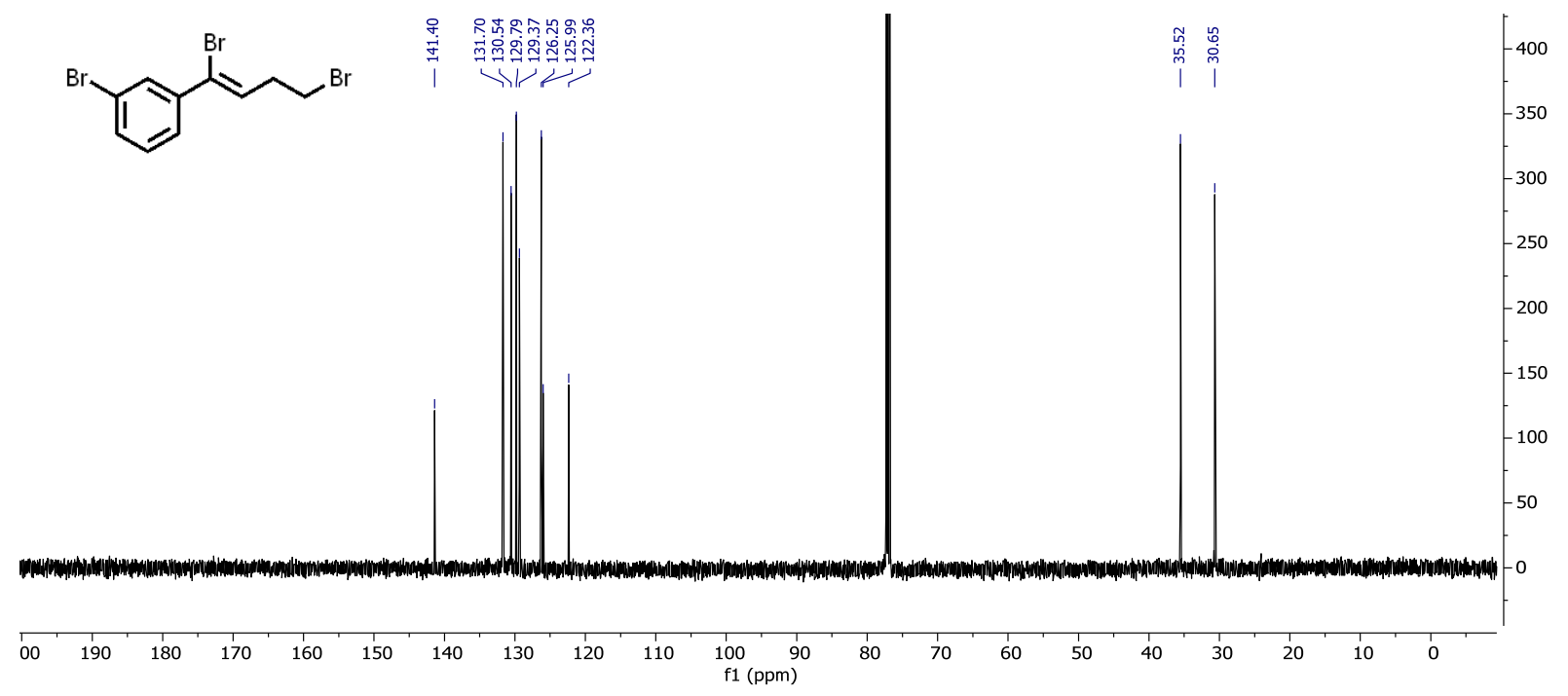




\section{Synthesis of Chiral Catalysts}

\section{General catalyst synthesis procedure:}<smiles>[R]OC(=O)c1cc(O[C@@H](Cc2ccc([R])cc2)C(=O)O[R])c(I)c(O[C@@H](Cc2ccc(C[C@@H](O)C(=O)O[R])cc2)C(=O)O[R])c1</smiles>

The following procedure was modified from one reported by Banik, et. al. ${ }^{5}$ Diisopropyl azodicarboxylate ( $7.66 \mathrm{~mL}$, $46.0 \mathrm{mmol}, 2.30$ equiv) was added dropwise via syringe over 10 minutes to a stirred suspension of methyl 3,5dihydroxy-4-iodobenzoate ( 1 equiv), triphenylphosphine (2.70 equiv), and the appropriate $\alpha$-hydroxy ester (2.10 equiv) in tetrahydrofuran $(0.1 \mathrm{M})$ at $0{ }^{\circ} \mathrm{C}$. The reaction mixture was warmed to room temperature. After 12 hours, the reaction mixture was concentrated under reduced pressure. The residue was purified by flash column chromatography (DCM), followed by an additional flash column chromatography purification (Hexanes: $\mathrm{Et}_{2} \mathrm{O}$ ).<smiles>CC(=O)c1cc(OC(Cc2ccc(C(C)(C)C)cc2)Cc2ccc(C(C)(C)C)cc2)c(I)c(O[C@@H](CC(=O)OCc2ccccc2)C(=O)OCc2ccccc2)c1</smiles>

dibenzyl 2,2'-((2-iodo-5-(methoxycarbonyl)-1,3-phenylene)bis(oxy))(2R,2'R)-bis(3-(4-(tertbutyl)phenyl)propanoate). $\mathbf{3 b}$ was isolated as a crystalline, white powder.

${ }^{1} \mathrm{H}$ NMR $\left(500 \mathrm{MHz}, \mathrm{CDCl}_{3}\right) \delta 7.38-7.11(\mathrm{~m}, 28 \mathrm{H}), 6.84(\mathrm{~s}, 2 \mathrm{H}), 5.09(\mathrm{~d}, J=1.5 \mathrm{~Hz}, 5 \mathrm{H}), 4.94(\mathrm{dd}, J=8.0,4.6 \mathrm{~Hz}$, $2 \mathrm{H}), 3.75(\mathrm{~s}, 3 \mathrm{H}), 3.39-3.21(\mathrm{~m}, 6 \mathrm{H}), 1.29(\mathrm{~s}, 16 \mathrm{H})$.

${ }^{13} \mathrm{C}$ NMR $\left(126 \mathrm{MHz}, \mathrm{CDCl}_{3}\right) \delta$ 170.03, 165.77, 157.91, 149.88, 135.01, 132.76, 130.48, 129.57, $129.31-128.07$ (m), 125.36, 106.40, 86.15, 78.65, 67.13, 52.31, 38.43, 34.46, 31.37.

HRMS (ESI): for $\mathrm{C}_{48} \mathrm{H}_{52} \mathrm{IO}_{8},[\mathrm{M}+\mathrm{H}]^{+}$calculated $\mathrm{m} / z=883.2701$, found $\mathrm{m} / z=883.2691$.

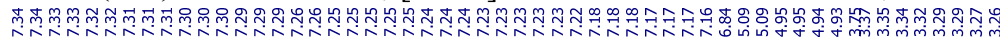

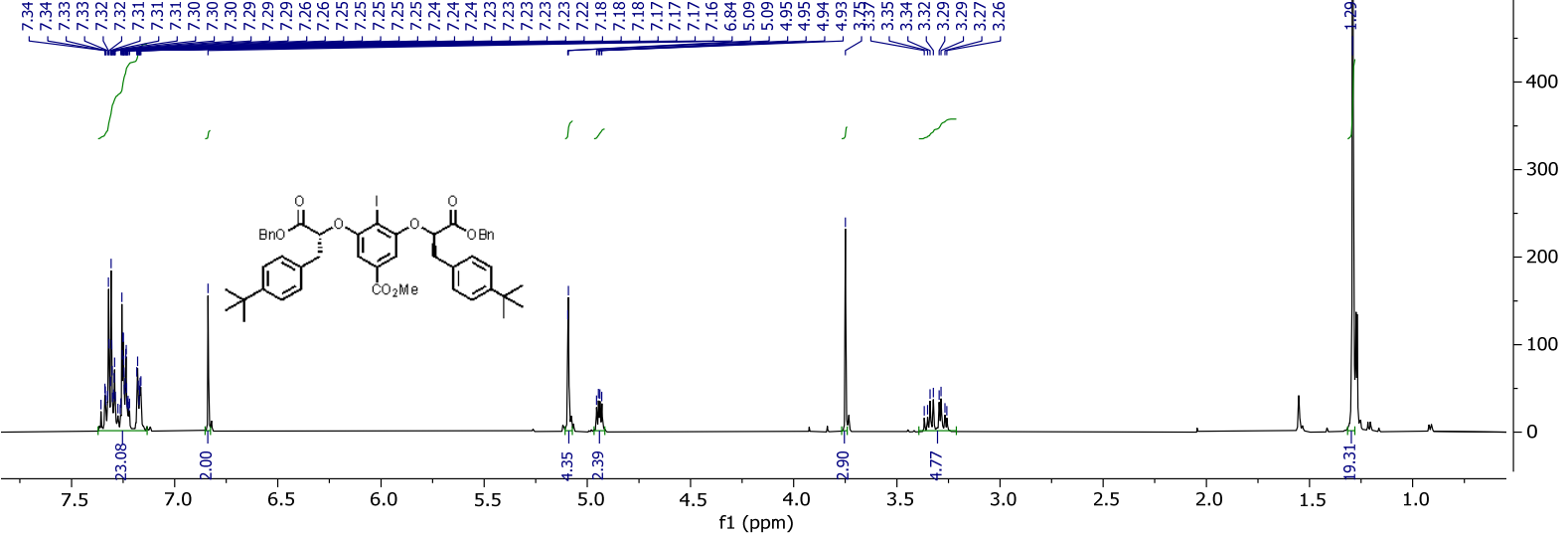




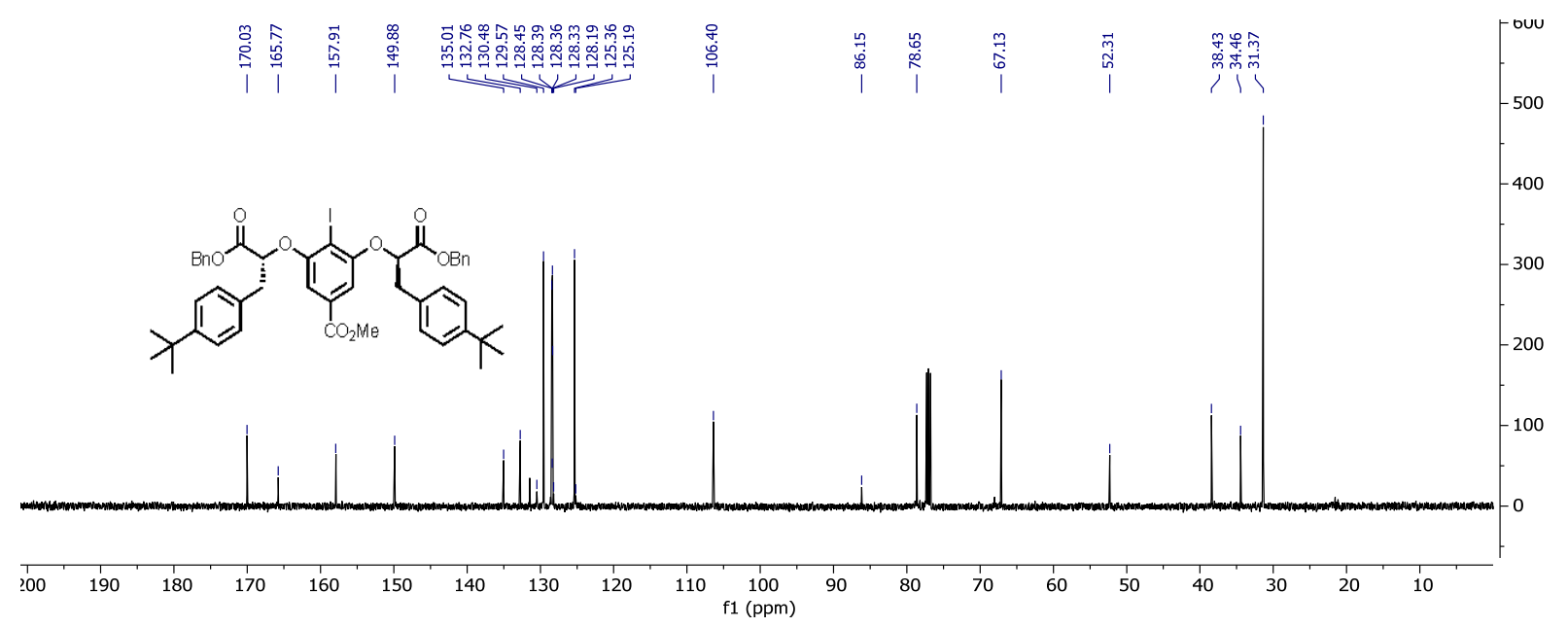<smiles>CC(=O)c1cc(O[C@H](Cc2ccc(C(C)(C)C)cc2)C(=O)OCc2ccc(C(C)(C)C)cc2)c(I)c(O[C@H](Cc2ccc(S(F)(F)(F)(F)F)cc2)C(=O)OCc2ccc(C(C)(C)C)cc2)c1</smiles>

bis(4-(pentafluoro- $\lambda^{6}$-sulfanyl)benzyl) 2,2'-((2-iodo-5-(methoxycarbonyl)-1,3-phenylene)bis(oxy))(2R,2'R)-bis(3-(4(tert-butyl)phenyl)propanoate). $\mathbf{3 c}$ was isolated as a crystalline, white powder.

${ }^{1} \mathrm{H}$ NMR $\left(500 \mathrm{MHz}, \mathrm{CDCl}_{3}\right) \delta 7.63-7.53(\mathrm{~m}, 4 \mathrm{H}), 7.40-7.29(\mathrm{~m}, 8 \mathrm{H}), 7.21(\mathrm{~d}, J=8.3 \mathrm{~Hz}, 4 \mathrm{H}), 6.80(\mathrm{~s}, 2 \mathrm{H}), 5.15$ $-5.05(\mathrm{~m}, 4 \mathrm{H}), 4.97(\mathrm{dd}, J=7.9,4.6 \mathrm{~Hz}, 2 \mathrm{H}), 3.74(\mathrm{~s}, 3 \mathrm{H}), 3.36(\mathrm{dd}, J=14.0,7.9 \mathrm{~Hz}, 2 \mathrm{H}), 3.30(\mathrm{dd}, J=14.0,4.6$ $\mathrm{Hz}, 2 \mathrm{H}), 1.27$ (s, 18H).

${ }^{13} \mathrm{C}$ NMR $\left(126 \mathrm{MHz}, \mathrm{CDCl}_{3}\right) \delta 169.81,165.49,157.84,153.58$ (d, $\left.J=17.0 \mathrm{~Hz}\right), 150.14,138.77,132.49,131.50$, $129.58,128.17,126.31-125.82(\mathrm{~m}), 125.42,106.33,86.05,78.55,65.51,52.33,38.42,34.45,31.31$.

${ }^{19} \mathrm{~F}$ NMR $\left(471 \mathrm{MHz}, \mathrm{CDCl}_{3}\right) \delta 84.16(\mathrm{p}, J=150.3,149.8 \mathrm{~Hz}, 1 \mathrm{~F}), 62.77(\mathrm{~d}, J=149.6 \mathrm{~Hz}, 4 \mathrm{~F})$.

HRMS (ESI): for $\mathrm{C}_{48} \mathrm{H}_{50} \mathrm{~F}_{10} \mathrm{IO}_{8} \mathrm{~S}_{2},[\mathrm{M}+\mathrm{H}]^{+}$calculated $m / z=1135.1827$, found $m / z=1135.1808$.

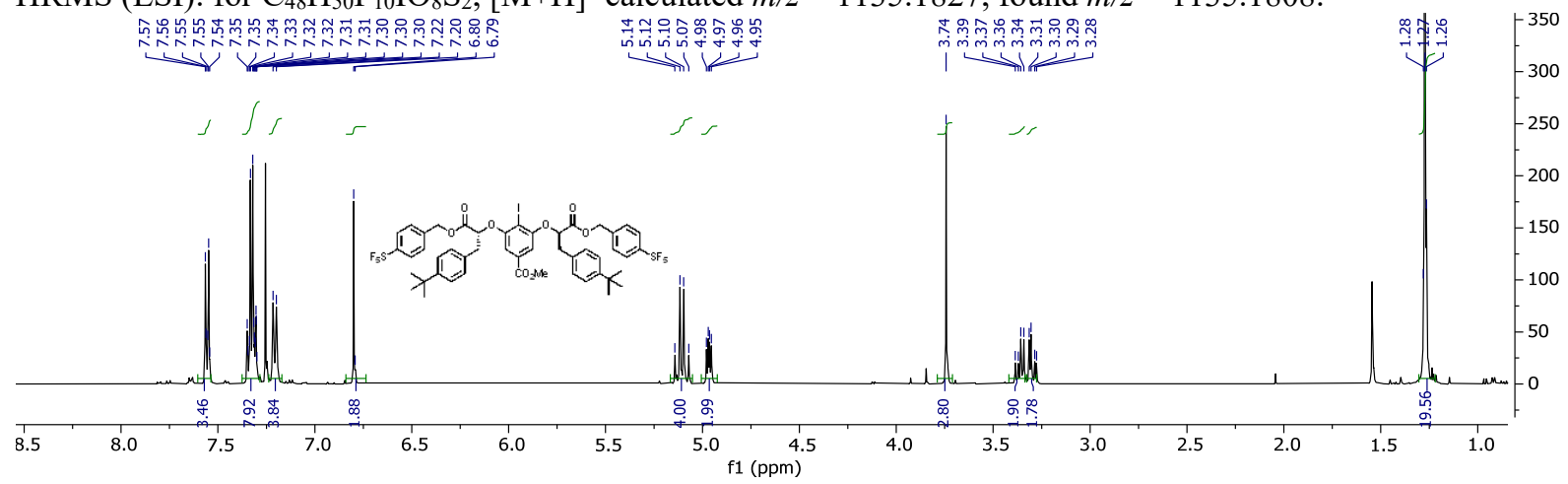




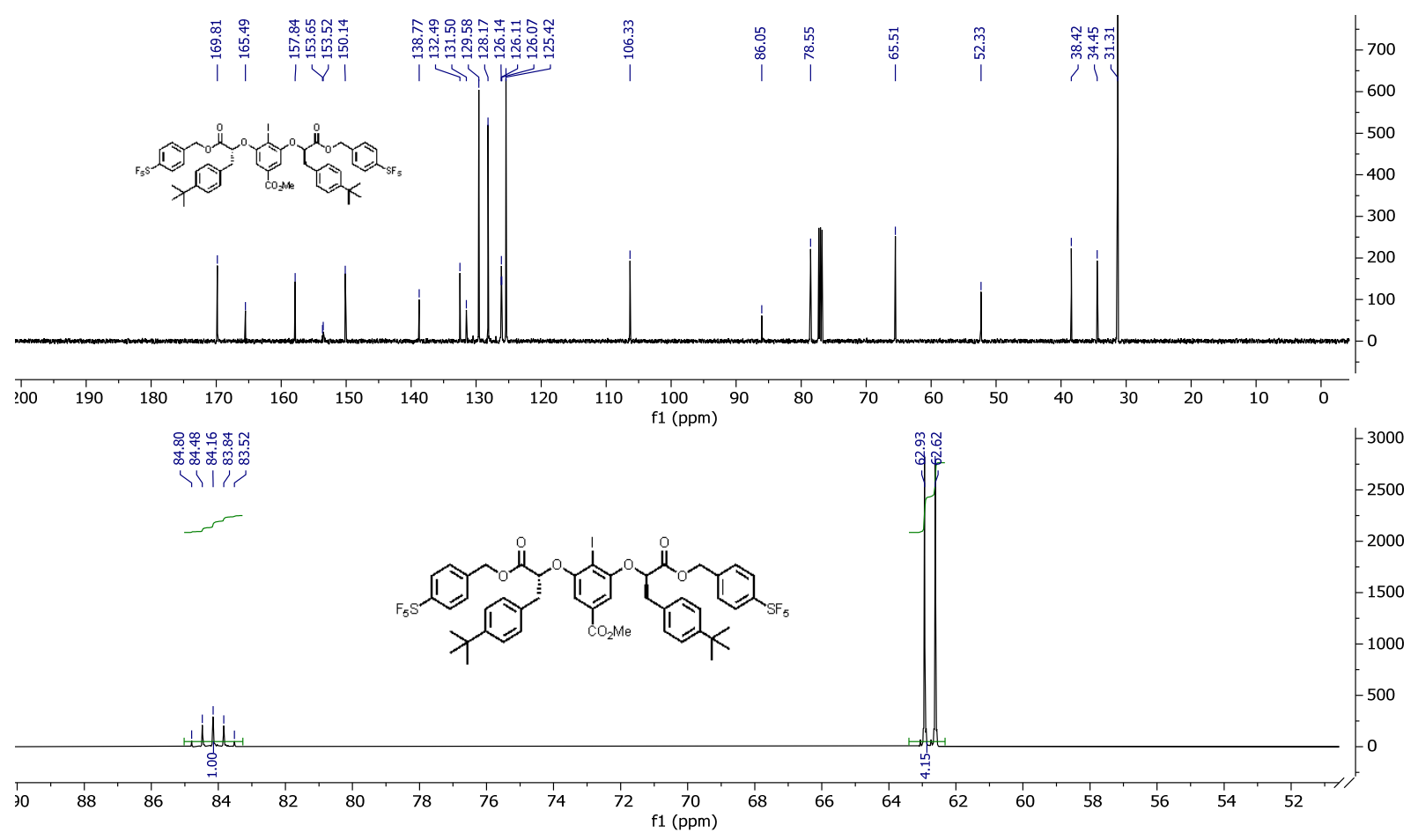<smiles>COC(=O)c1cc(O[C@@H](Cc2ccccc2)C(=O)OC)c(I)c(O[C@H](Cc2ccccc2)C(=O)OC)c1</smiles>

dimethyl 2,2'-((2-iodo-5-(methoxycarbonyl)-1,3-phenylene)bis(oxy))(2R,2'R)-bis(3-phenylpropanoate) ${ }^{1} \mathrm{H}$ NMR $\left(500 \mathrm{MHz}, \mathrm{CDCl}_{3}\right) \delta 7.46-7.39(\mathrm{~m}, 4 \mathrm{H}), 7.36-7.28(\mathrm{~m}, 4 \mathrm{H}), 7.28-7.22(\mathrm{~m}, 2 \mathrm{H}), 6.87(\mathrm{~s}, 2 \mathrm{H}), 4.93(\mathrm{dd}$, $J=7.8,4.6 \mathrm{~Hz}, 2 \mathrm{H}), 3.83(\mathrm{~s}, 3 \mathrm{H}), 3.69(\mathrm{~s}, 6 \mathrm{H}), 3.39-3.28(\mathrm{~m}, 4 \mathrm{H}) .3 \mathrm{~d}$ was isolated as a crystalline, white powder. ${ }^{13} \mathrm{C}$ NMR $\left(126 \mathrm{MHz}, \mathrm{CDCl}_{3}\right) \delta 170.51,165.97,158.03,135.90,131.60,129.89,128.45,127.12,106.40,86.15$, 78.69, 52.42, 39.01, 29.72.

HRMS (ESI): for $\mathrm{C}_{28} \mathrm{H}_{28} \mathrm{IO}_{8},[\mathrm{M}+\mathrm{H}]^{+}$calculated $m / z=619.0823$, found $m / z=619.0819$.

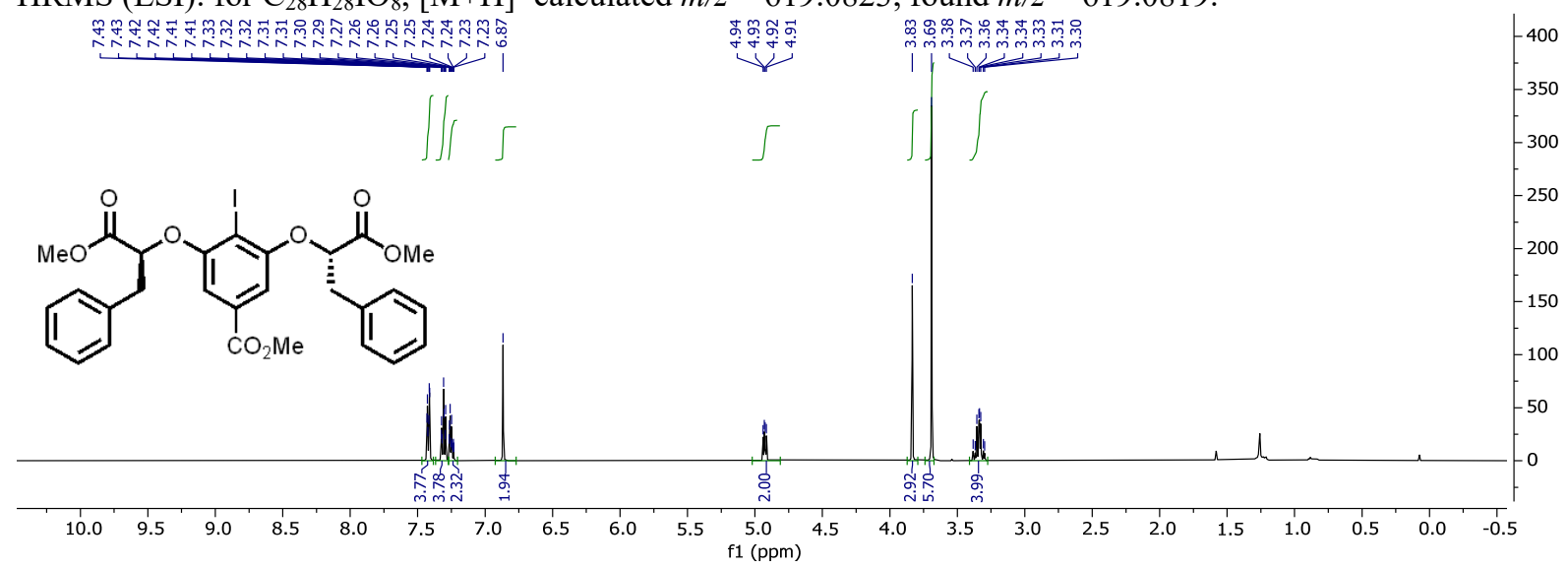




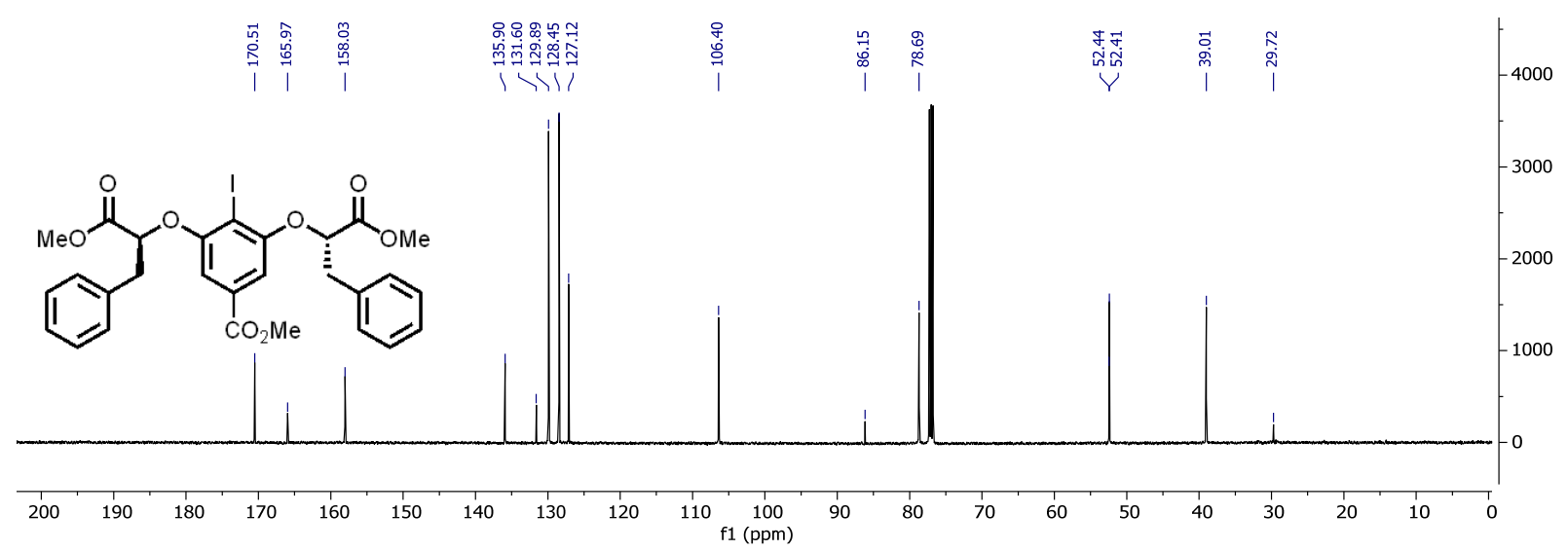<smiles>COC(=O)c1cc(O[C@@H](Cc2ccc(C)cc2)C(=O)OC)c(I)c(O[C@H](Cc2ccc(C)cc2)C(=O)OC)c1</smiles>

dimethyl 2,2'-((2-iodo-5-(methoxycarbonyl)-1,3-phenylene)bis(oxy))(2S,2'S)-bis(3-(p-tolyl)propanoate). 3e was isolated as a crystalline, white powder.

${ }^{1} \mathrm{H}$ NMR $\left(500 \mathrm{MHz}, \mathrm{CDCl}_{3}\right) \delta 7.32(\mathrm{~d}, J=8.0 \mathrm{~Hz}, 4 \mathrm{H}), 7.15-7.10(\mathrm{~m}, 4 \mathrm{H}), 6.87(\mathrm{~s}, 2 \mathrm{H}), 4.91(\mathrm{dd}, J=7.7,4.6 \mathrm{~Hz}$, $2 \mathrm{H}), 3.85(\mathrm{~d}, J=0.5 \mathrm{~Hz}, 3 \mathrm{H}), 3.71(\mathrm{~d}, J=0.5 \mathrm{~Hz}, 6 \mathrm{H}), 3.39-3.22(\mathrm{~m}, 5 \mathrm{H}), 2.33(\mathrm{~s}, 6 \mathrm{H})$.

${ }^{13} \mathrm{C}$ NMR $\left(126 \mathrm{MHz}, \mathrm{CDCl}_{3}\right) \delta 170.57,165.99,158.05,136.65,132.80,131.56,129.76,129.14,106.34,86.15$, 78.81, 52.44, 52.39, 38.60, 21.12.

HRMS (ESI): for $\mathrm{C}_{30} \mathrm{H}_{32} \mathrm{IO}_{8},[\mathrm{M}+\mathrm{H}]^{+}$calculated $\mathrm{m} / z=647.1136$, found $\mathrm{m} / z=647.1129$.

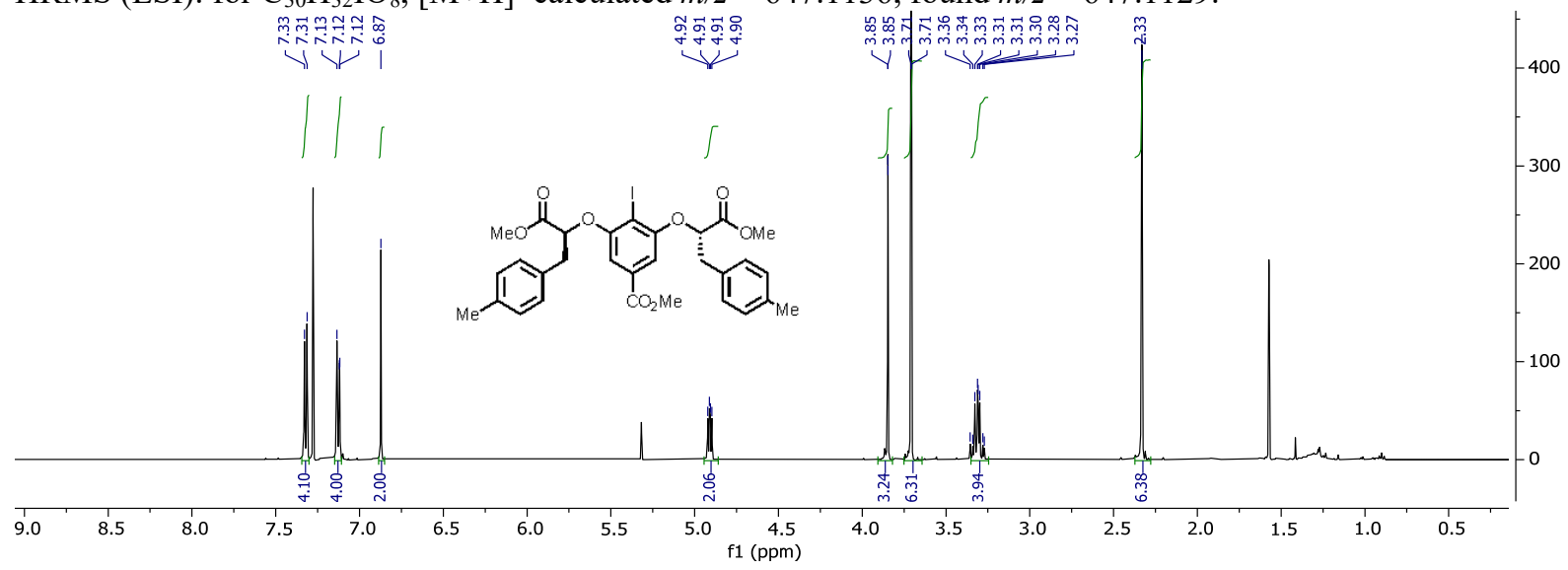



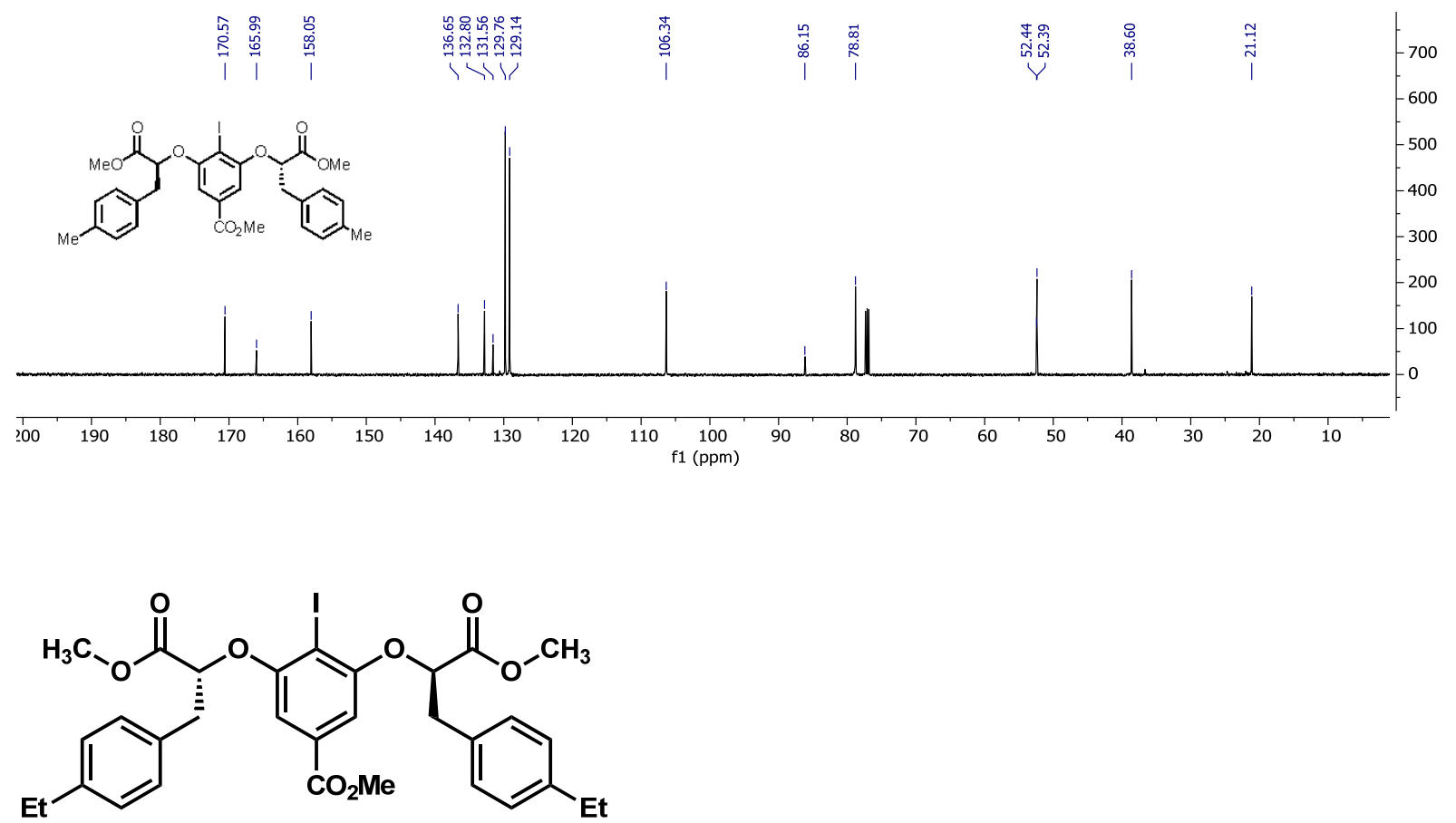

dimethyl 2,2'-((2-iodo-5-(methoxycarbonyl)-1,3-phenylene)bis(oxy))(2R,2'R)-bis(3-(4-ethylphenyl)propanoate). 3e was isolated as a crystalline, white powder.

${ }^{1} \mathrm{H}$ NMR $\left(500 \mathrm{MHz}, \mathrm{CDCl}_{3}\right) \delta 7.33(\mathrm{~d}, J=8.1 \mathrm{~Hz}, 3 \mathrm{H}), 7.14(\mathrm{~d}, J=7.8 \mathrm{~Hz}, 4 \mathrm{H}), 6.86(\mathrm{~s}, 2 \mathrm{H}), 4.90(\mathrm{dd}, J=7.9,4.5$ $\mathrm{Hz}, 2 \mathrm{H}), 3.83(\mathrm{~s}, 2 \mathrm{H}), 3.69$ (s, 6H), $3.36-3.23(\mathrm{~m}, 4 \mathrm{H}), 2.62$ (q, $J=7.6 \mathrm{~Hz}, 5 \mathrm{H}), 1.22$ (t, $J=7.6 \mathrm{~Hz}, 5 \mathrm{H})$.

${ }^{13} \mathrm{C} \mathrm{NMR}\left(126 \mathrm{MHz}, \mathrm{CDCl}_{3}\right) \delta 170.60,165.98,158.06,143.02,133.09,131.57,129.83,127.95,106.35,86.17$, $78.82,52.44,52.40,38.65,28.52,15.55$.

HRMS (ESI): for $\mathrm{C}_{32} \mathrm{H}_{36} \mathrm{IO}_{8},[\mathrm{M}+\mathrm{H}]^{+}$calculated $\mathrm{m} / z=675.1149$, found $\mathrm{m} / z=675.1442$.

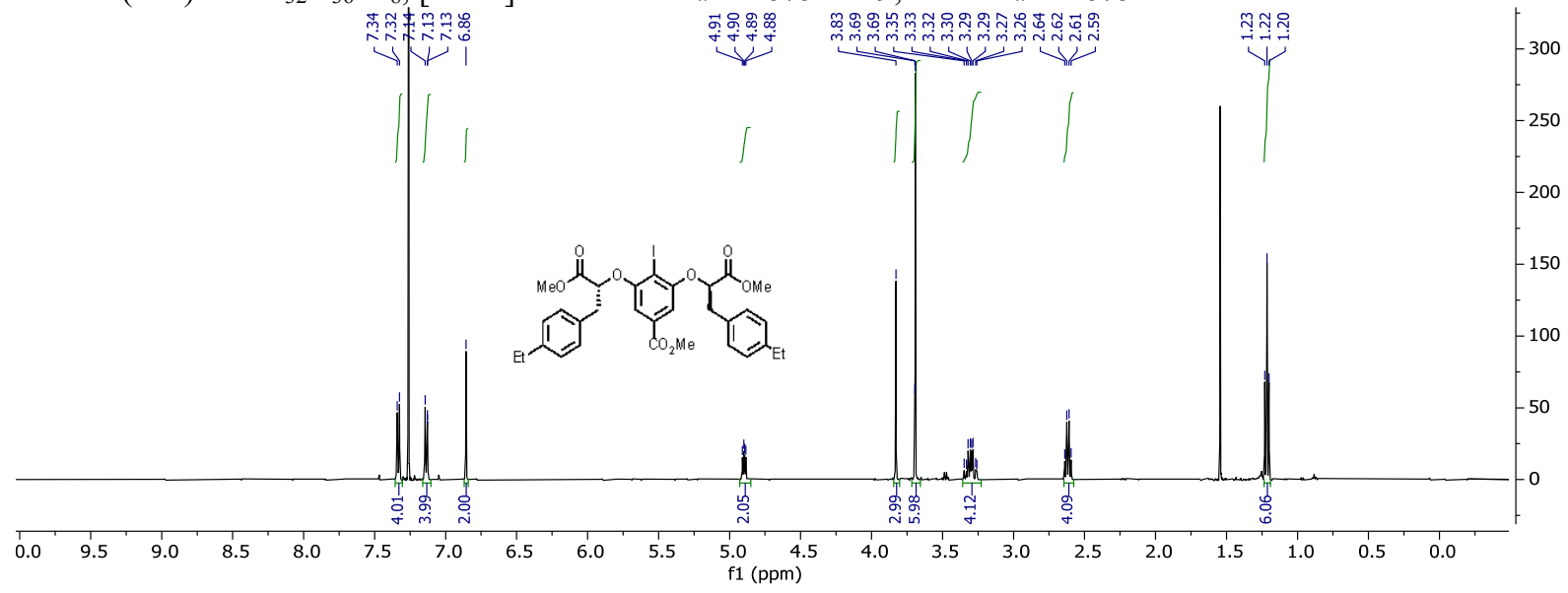




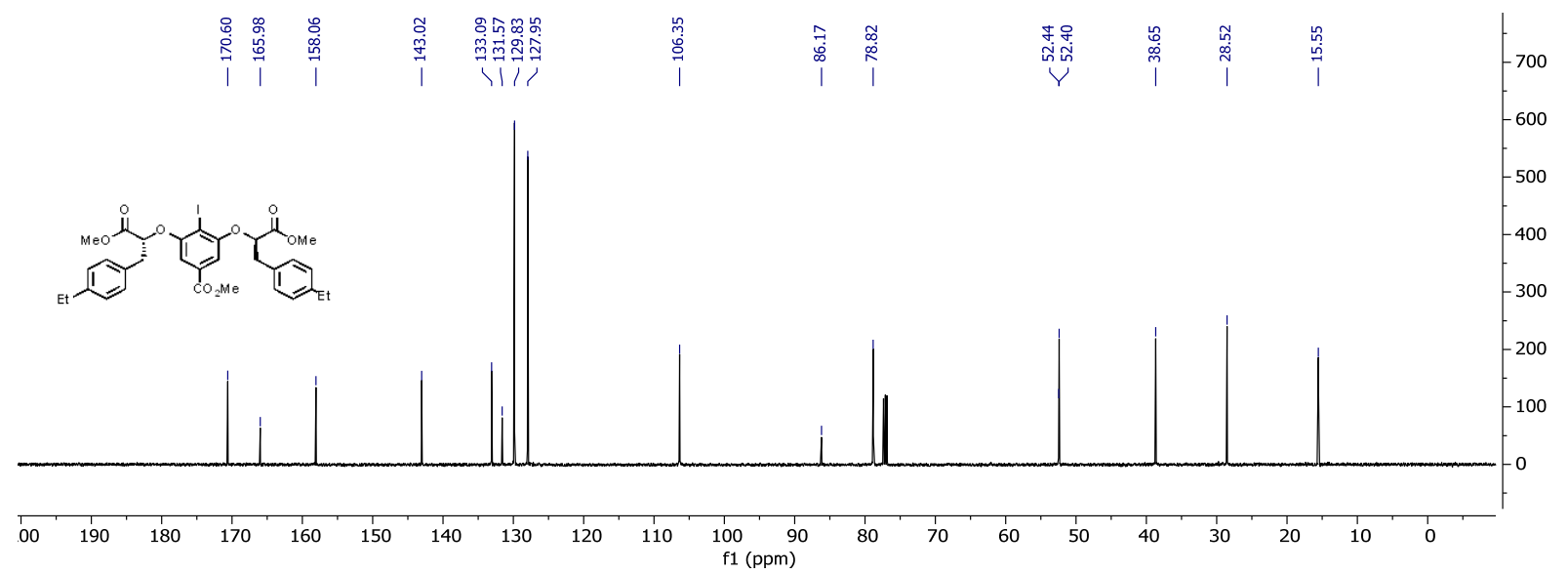<smiles>COC(=O)c1cc(O[C@@H](Cc2ccc(C(C)C)cc2)C(=O)OC)c(I)c(O[C@H](Cc2ccc(C(C)C)cc2)C(=O)OC)c1</smiles>

dimethyl 2,2'-((2-iodo-5-(methoxycarbonyl)-1,3-phenylene)bis(oxy))(2R,2'R)-bis(3-(4isopropylphenyl)propanoate). $3 \mathbf{f}$ was isolated as a crystalline, white powder.

${ }^{1} \mathrm{H}$ NMR $\left(500 \mathrm{MHz}, \mathrm{CDCl}_{3}\right) \delta 7.36(\mathrm{~d}, J=8.1 \mathrm{~Hz}, 4 \mathrm{H}), 7.18(\mathrm{~d}, J=8.1 \mathrm{~Hz}, 4 \mathrm{H}), 6.88(\mathrm{~s}, 2 \mathrm{H}), 4.92(\mathrm{dd}, J=8.0,4.4$ Hz, 2H), 3.85 (s, 3H), 3.71 (s, 6H), 3.32 (qd, $J=14.1,6.2 \mathrm{~Hz}, 4 \mathrm{H}), 2.89$ (p, $J=6.9 \mathrm{~Hz}, 2 \mathrm{H}), 1.25$ (d, $J=6.9 \mathrm{~Hz}$, $12 \mathrm{H})$.

${ }^{13} \mathrm{C}$ NMR $\left(126 \mathrm{MHz}, \mathrm{CDCl}_{3}\right) \delta 170.65,166.01,158.06,147.64,133.25,131.56,129.81,126.51,106.36,86.19$, $78.82,52.45,52.41,38.65,33.78,24.02$.

HRMS (ESI): for $\mathrm{C}_{34} \mathrm{H}_{40} \mathrm{IO}_{8},[\mathrm{M}+\mathrm{H}]^{+}$calculated $\mathrm{m} / z=703.1762$, found $\mathrm{m} / z=703.1751$.
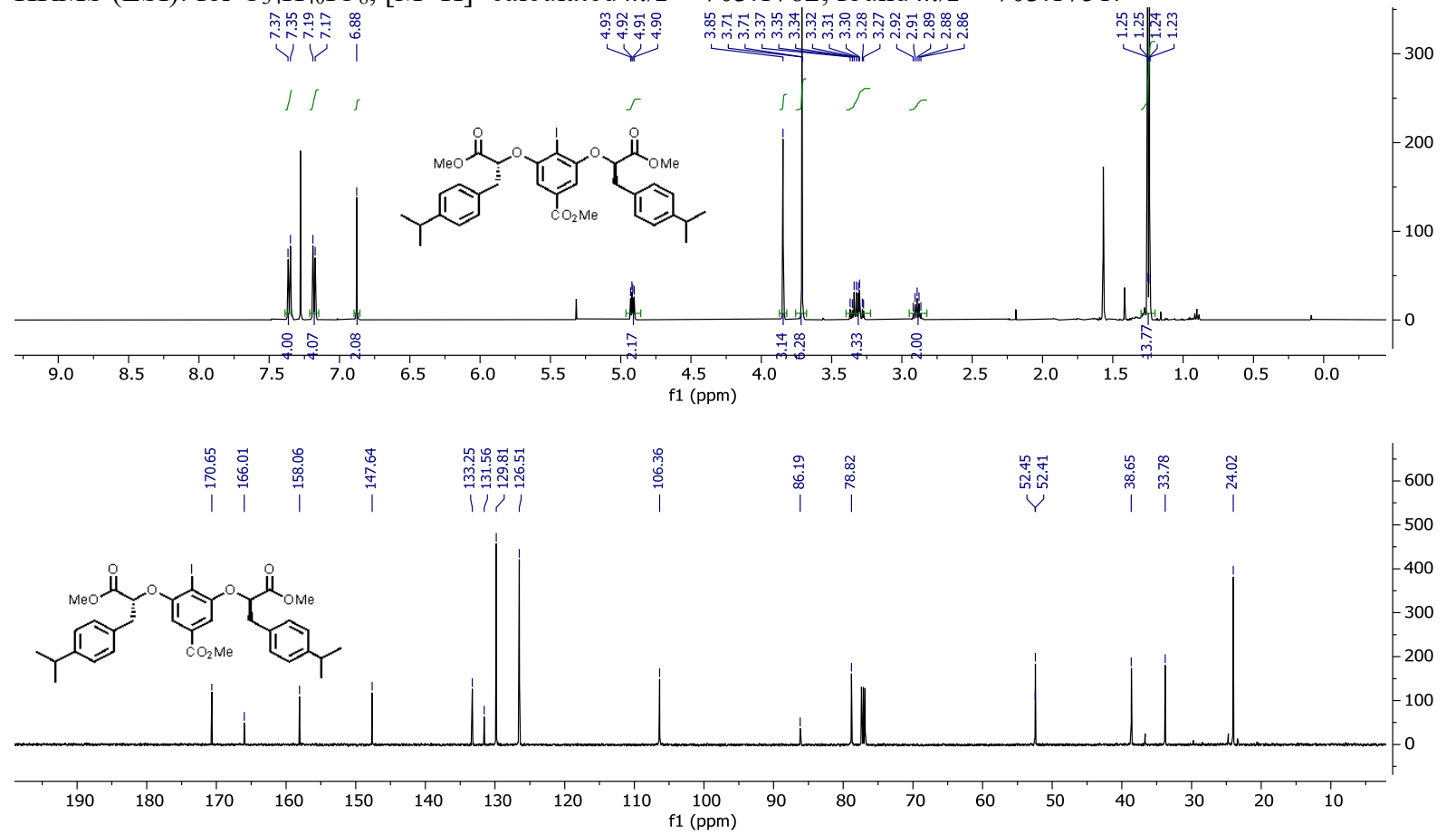
<smiles>COC(=O)c1cc(O[C@@H](Cc2ccc(C(C)(C)C)cc2)C(=O)OC)c(I)c(O[C@H](Cc2ccc(C(C)(C)C)cc2)C(=O)OC)c1</smiles>

dimethyl 2,2'-((2-iodo-5-(methoxycarbonyl)-1,3-phenylene)bis(oxy))(2R,2'R)-bis(3-(4-(tertbutyl)phenyl)propanoate). $\mathbf{3 g}$ was isolated as a crystalline, white powder.

${ }^{1} \mathrm{H}$ NMR $\left(500 \mathrm{MHz}, \mathrm{CDCl}_{3}\right) \delta 7.34(\mathrm{q}, J=8.3 \mathrm{~Hz}, 8 \mathrm{H}), 6.86(\mathrm{~s}, 2 \mathrm{H}), 4.91(\mathrm{dd}, J=8.2,4.3 \mathrm{~Hz}, 2 \mathrm{H}), 3.83(\mathrm{~s}, 3 \mathrm{H})$, $3.70(\mathrm{~s}, 6 \mathrm{H}), 3.31(\mathrm{qd}, J=14.1,6.2 \mathrm{~Hz}, 4 \mathrm{H}), 1.30(\mathrm{~s}, 18 \mathrm{H})$.

${ }^{13} \mathrm{C}$ NMR $\left(126 \mathrm{MHz}, \mathrm{CDCl}_{3}\right) \delta 170.65,165.97,158.03,149.90,132.90,131.54,129.52,125.36,106.32,86.17$, $78.75,52.42,52.40,38.52,34.46,31.36$.

HRMS (ESI): for $\mathrm{C}_{36} \mathrm{H}_{44} \mathrm{IO}_{8},[\mathrm{M}+\mathrm{H}]^{+}$calculated $\mathrm{m} / z=731.2075$, found $\mathrm{m} / z=731.2066$.
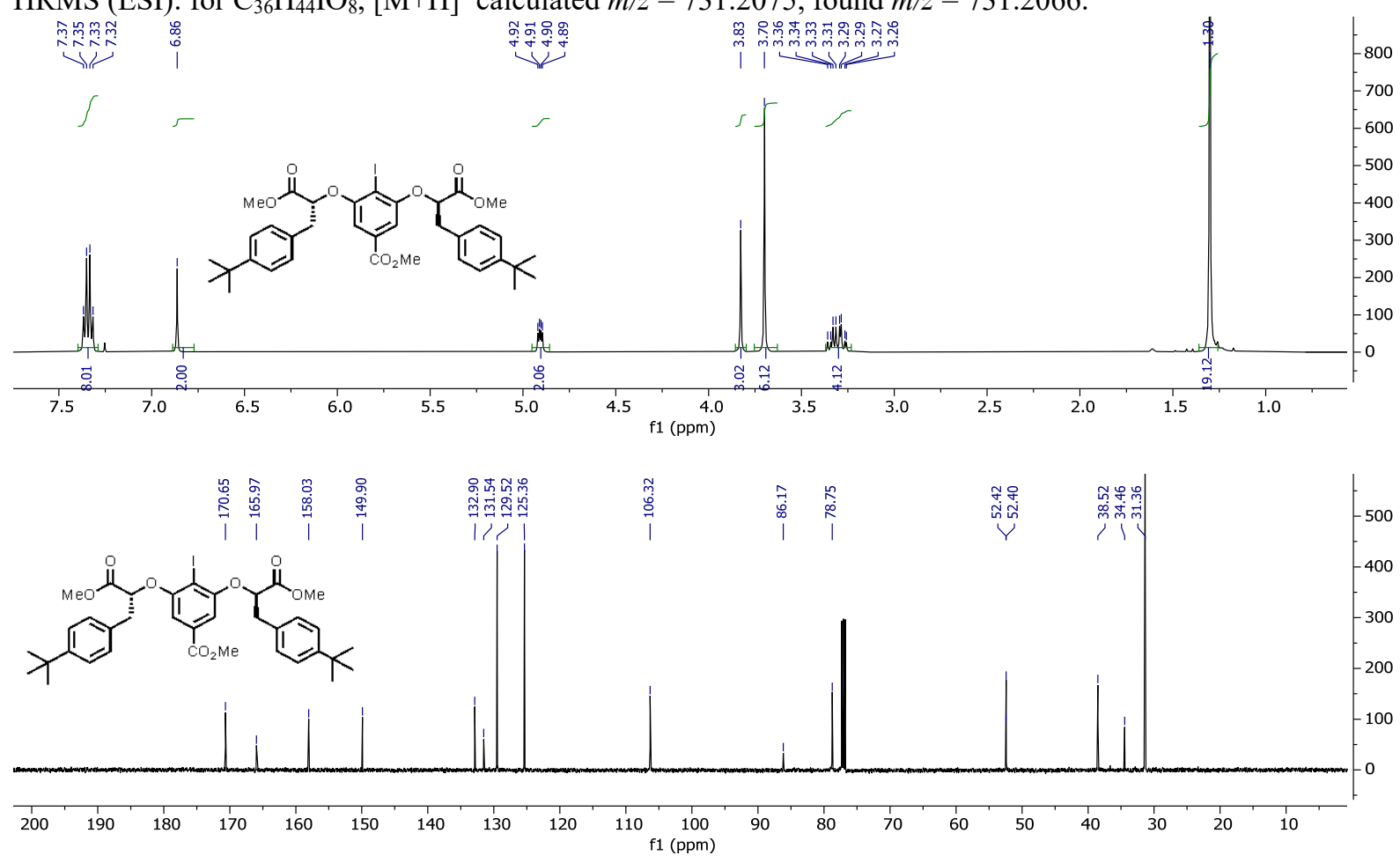<smiles>COC(=O)[C@H](Cc1ccc(Cl)cc1)Oc1cc(O[C@H](Cc2ccc(Cl)cc2)C(=O)OC)cc(O[C@H](Cc2ccccc2)C(=O)OC)c1I</smiles>

dimethyl 2,2'-((2-iodo-5-(methoxycarbonyl)-1,3-phenylene)bis(oxy))(2S,2'S)-bis(3-(4-adamantylphenyl)propanoate. $3 \mathbf{h}$ was isolated as a crystalline, white powder.

${ }^{1} \mathrm{H}$ NMR $\left(500 \mathrm{MHz}, \mathrm{CDCl}_{3}\right) \delta 7.36(\mathrm{~d}, J=8.3 \mathrm{~Hz}, 4 \mathrm{H}), 7.29(\mathrm{~d}, J=8.3 \mathrm{~Hz}, 4 \mathrm{H}), 6.85(\mathrm{~s}, 2 \mathrm{H}), 4.89(\mathrm{dd}, J=8.2,4.3$ $\mathrm{Hz}, 2 \mathrm{H}), 3.82(\mathrm{~s}, 3 \mathrm{H}), 3.69(\mathrm{~s}, 5 \mathrm{H}), 3.36-3.23(\mathrm{~m}, 5 \mathrm{H}), 2.08(\mathrm{~s}, 3 \mathrm{H}), 1.89$ (d, $J=3.0 \mathrm{~Hz}, 14 \mathrm{H}), 1.81-1.69(\mathrm{~m}$, $16 \mathrm{H})$. 
${ }^{13} \mathrm{C}$ NMR $\left(126 \mathrm{MHz}, \mathrm{CDCl}_{3}\right) \delta 170.67,165.98,158.05,150.16,132.97,131.54,129.58,124.95,106.35,86.19$, $78.80,52.43,52.40,43.18,38.58,36.83,36.00,28.98$.

HRMS (ESI): for $\mathrm{C}_{48} \mathrm{H}_{56} \mathrm{IO}_{8},[\mathrm{M}+\mathrm{H}]^{+}$calculated $\mathrm{m} / \mathrm{z}=887.3014$, found $\mathrm{m} / \mathrm{z}=887.3008$.
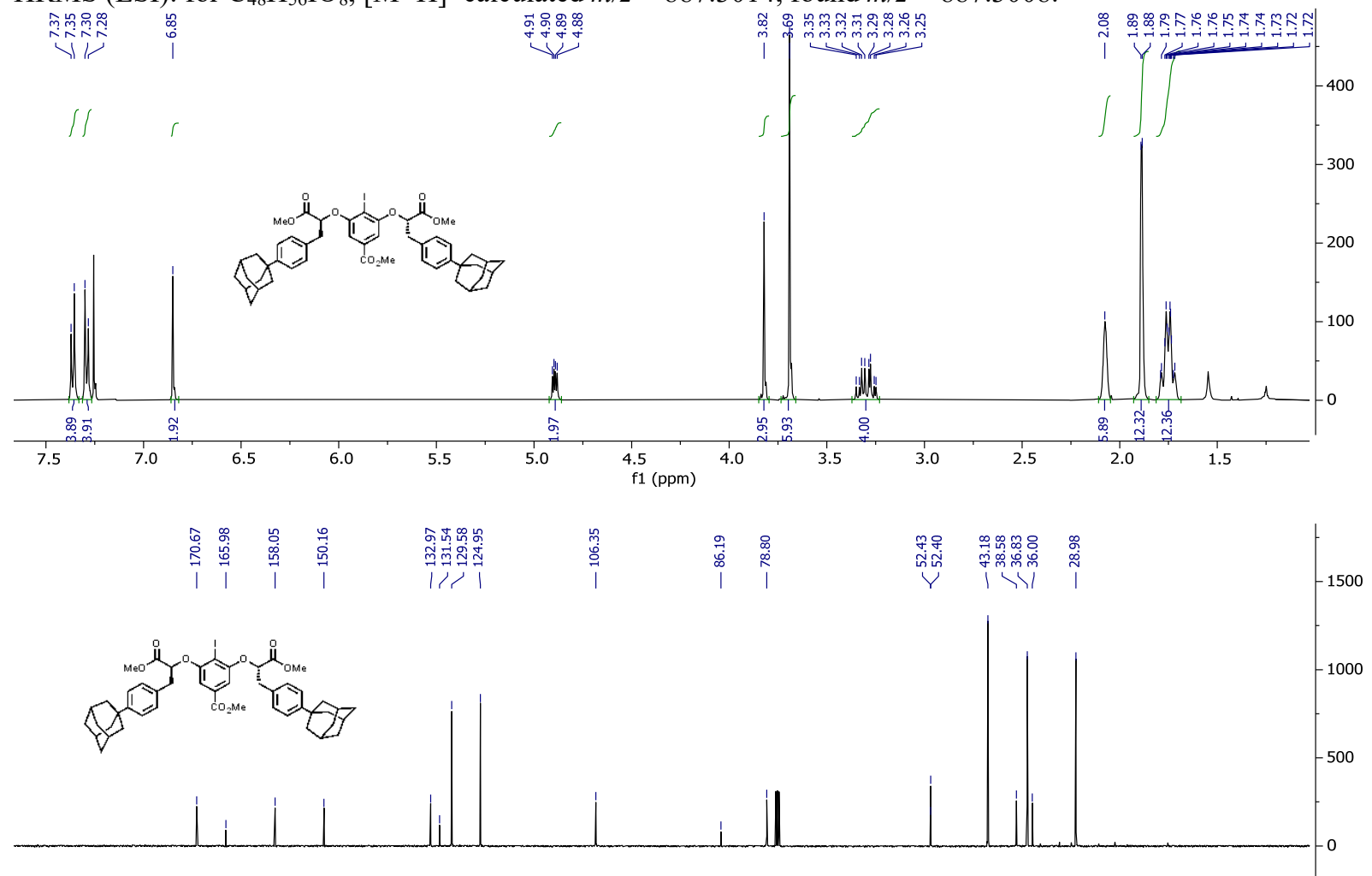

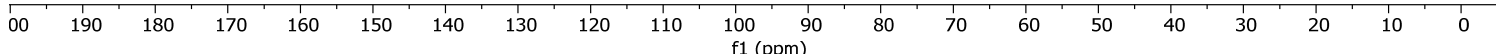<smiles>COC(=O)c1cc(O[C@@H](Cc2ccc(C(F)(F)F)cc2)C(=O)OC)c(I)c(O[C@H](Cc2ccc(C(F)(F)F)cc2)C(=O)OC)c1</smiles>

dimethyl 2,2'-((2-iodo-5-(methoxycarbonyl)-1,3-phenylene)bis(oxy))(2S,2'S)-bis(3-(4(trifluoromethyl)phenyl)propanoate). 3i was isolated as a crystalline, white powder.

${ }^{1} \mathrm{H} \mathrm{NMR}\left(500 \mathrm{MHz}, \mathrm{CDCl}_{3}\right) \delta 7.56(\mathrm{~d}, J=1.6 \mathrm{~Hz}, 7 \mathrm{H}), 6.86(\mathrm{~s}, 2 \mathrm{H}), 4.95(\mathrm{dd}, J=7.2,4.9 \mathrm{~Hz}, 2 \mathrm{H}), 3.84(\mathrm{~s}, 3 \mathrm{H})$, $3.71(\mathrm{~s}, 6 \mathrm{H}), 3.47-3.35(\mathrm{~m}, 3 \mathrm{H})$.

${ }^{13} \mathrm{C}$ NMR $\left(126 \mathrm{MHz}, \mathrm{CDCl}_{3}\right) \delta 170.00,165.78,157.83,139.92,131.73,130.31,129.48(\mathrm{q}, J=32.3 \mathrm{~Hz}), 125.36(\mathrm{q}$, $J=3.8 \mathrm{~Hz}), 124.17(\mathrm{q}, J=272.6,272.0,271.2 \mathrm{~Hz}), 106.45,85.91,78.03,52.56,52.50,38.65$.

${ }^{19} \mathrm{~F}$ NMR $\left(471 \mathrm{MHz}, \mathrm{CDCl}_{3}\right) \delta-65.09$.

HRMS (ESI): for $\mathrm{C}_{30} \mathrm{H}_{26} \mathrm{~F}_{6} \mathrm{IO}_{8},[\mathrm{M}+\mathrm{H}]^{+}$calculated $m / z=755.0571$, found $m / z=755.0561$. 

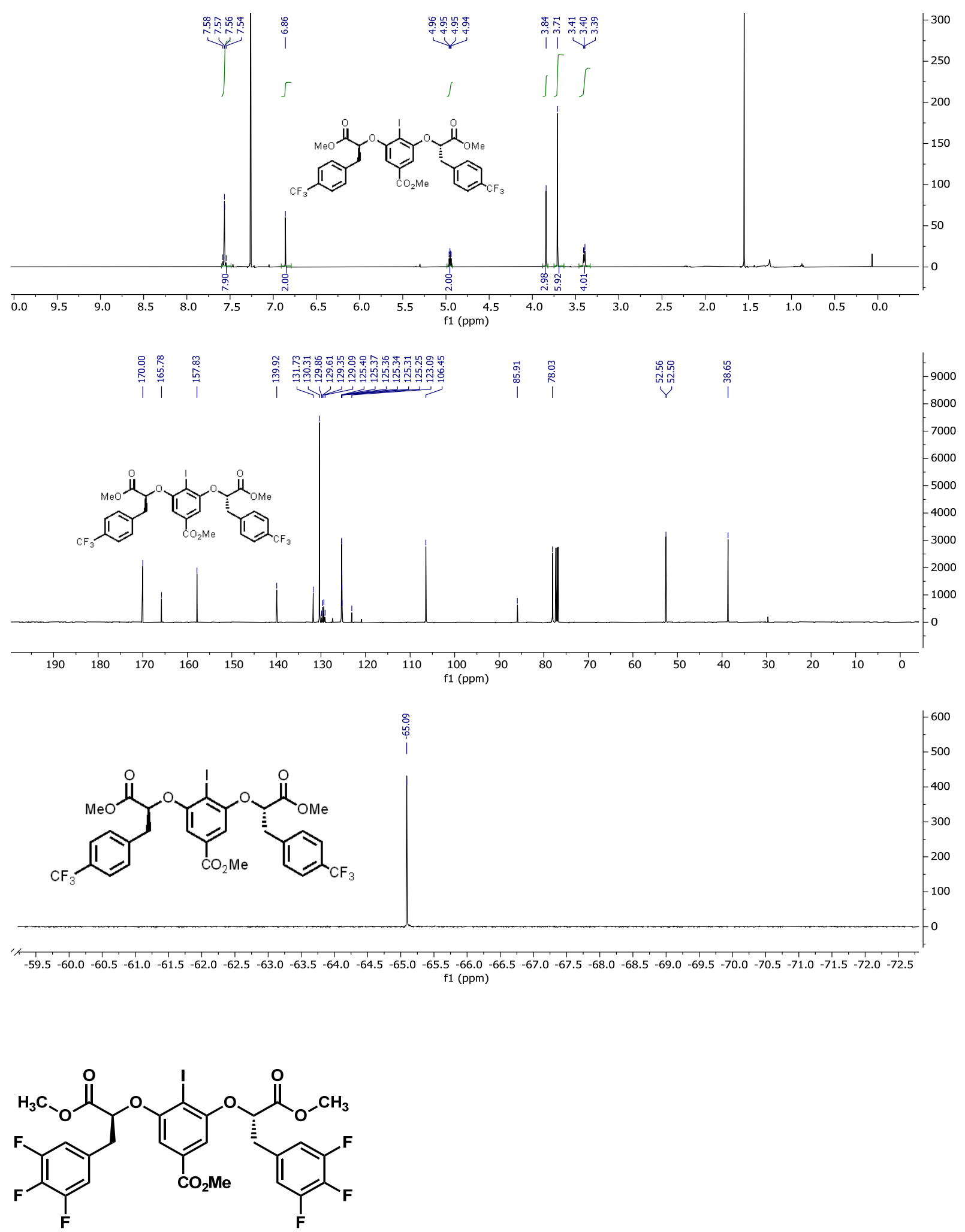

dimethyl 2,2'-((2-iodo-5-(methoxycarbonyl)-1,3-phenylene)bis(oxy))(2S,2'S)-bis(3-(3,4,5-

trifluorophenyl)propanoate). $\mathbf{3} \mathbf{j}$ was isolated as a crystalline, white powder.

${ }^{1} \mathrm{H}$ NMR $\left(500 \mathrm{MHz}, \mathrm{CDCl}_{3}\right) \delta 7.11(\mathrm{dd}, J=8.2,6.4 \mathrm{~Hz}, 4 \mathrm{H}), 6.89(\mathrm{~s}, 2 \mathrm{H}), 4.93(\mathrm{t}, J=5.9 \mathrm{~Hz}, 2 \mathrm{H}), 3.88(\mathrm{~s}, 3 \mathrm{H})$, $3.74(\mathrm{~s}, 6 \mathrm{H}), 3.30(\mathrm{~d}, J=5.8 \mathrm{~Hz}, 4 \mathrm{H})$. 
${ }^{13} \mathrm{C}$ NMR $\left(126 \mathrm{MHz}, \mathrm{CDCl}_{3}\right) \delta 169.65,165.70,157.74,150.85(\mathrm{ddd}, J=249.5,9.7,3.8 \mathrm{~Hz}), 138.98(\mathrm{dt}, J=250.6$, $15.3 \mathrm{~Hz}), 132.01$ (td, $J=7.8,4.6 \mathrm{~Hz}), 131.77,114.43-113.77$ (m), 106.44, 85.54, 77.59, 52.59, 52.50, 38.00 .

${ }^{19} \mathrm{~F}$ NMR $\left(471 \mathrm{MHz}, \mathrm{CDCl}_{3}\right) \delta-134.58(\mathrm{~d}, J=20.5 \mathrm{~Hz}, 4 \mathrm{~F}),-162.35(\mathrm{t}, J=20.6 \mathrm{~Hz}, 2 \mathrm{~F})$.

HRMS (ESI): for $\mathrm{C}_{28} \mathrm{H}_{22} \mathrm{~F}_{6} \mathrm{IO}_{8},[\mathrm{M}+\mathrm{H}]^{+}$calculated $m / z=727.0258$, found $m / z=727.0247$.
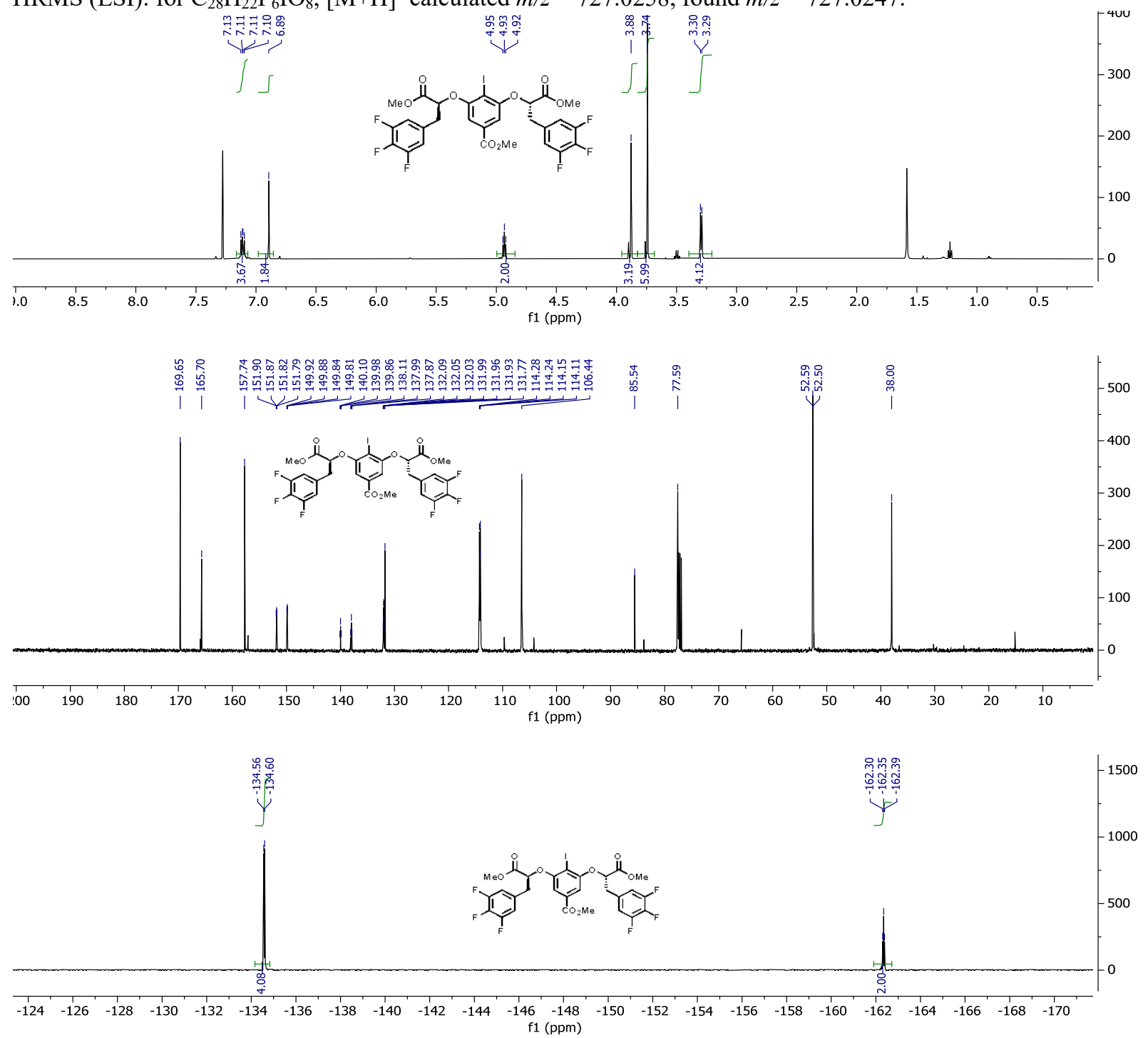<smiles>COC(=O)c1cc(O[C@@H](Cc2ccc(F)cc2)C(=O)OC)c(I)c(O[C@H](Cc2ccc(F)cc2)C(=O)OC)c1</smiles>

dimethyl 2,2'-((2-iodo-5-(methoxycarbonyl)-1,3-phenylene)bis(oxy))(2S,2'S)-bis(3-(4-fluorophenyl)propanoate). 3k was isolated as a crystalline, white powder.

${ }^{1} \mathrm{H} \mathrm{NMR}\left(500 \mathrm{MHz}, \mathrm{CDCl}_{3}\right) \delta 7.38(\mathrm{dd}, J=8.6,5.4 \mathrm{~Hz}, 4 \mathrm{H}), 6.99(\mathrm{t}, J=8.7 \mathrm{~Hz}, 3 \mathrm{H}), 6.86(\mathrm{~s}, 2 \mathrm{H}), 4.90(\mathrm{dd}, J=7.2$, $4.9 \mathrm{~Hz}, 3 \mathrm{H}), 3.84$ (s, 3H), 3.69 (s, 6H), $3.38-3.24(\mathrm{~m}, 4 \mathrm{H})$. 
${ }^{13} \mathrm{C}$ NMR $\left(126 \mathrm{MHz}, \mathrm{CDCl}_{3}\right) \delta 170.26,165.88,163.05,161.10,157.94,131.67,131.47$ (d, $\left.J=8.0 \mathrm{~Hz}\right), 115.27(\mathrm{~d}, J$ $=21.2 \mathrm{~Hz}), 106.41,86.00,78.49,52.48,52.45,38.12$.

${ }^{19} \mathrm{~F}$ NMR (471 MHz, $\left.\mathrm{CDCl}_{3}\right) \delta-115.72$.

HRMS (ESI): for $\mathrm{C}_{28} \mathrm{H}_{26} \mathrm{~F}_{2} \mathrm{IO}_{8}$, $[\mathrm{M}+\mathrm{H}]^{+}$calculated $m / z=655.0635$, found $m / z=655.0630$.

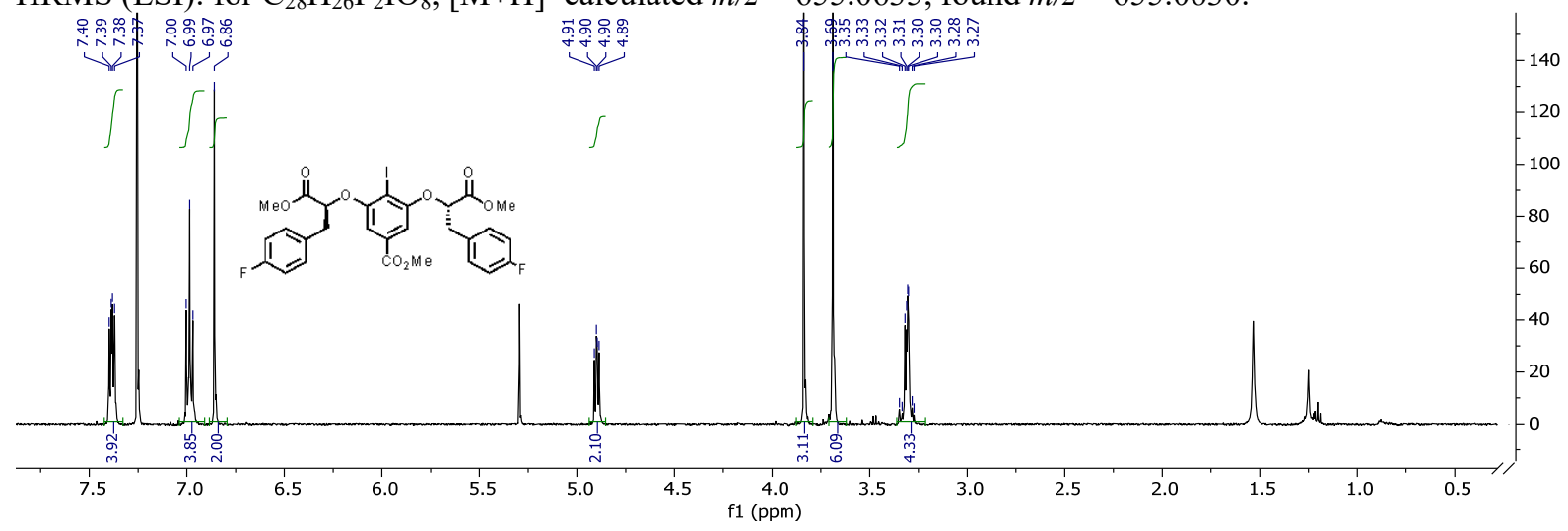

茖
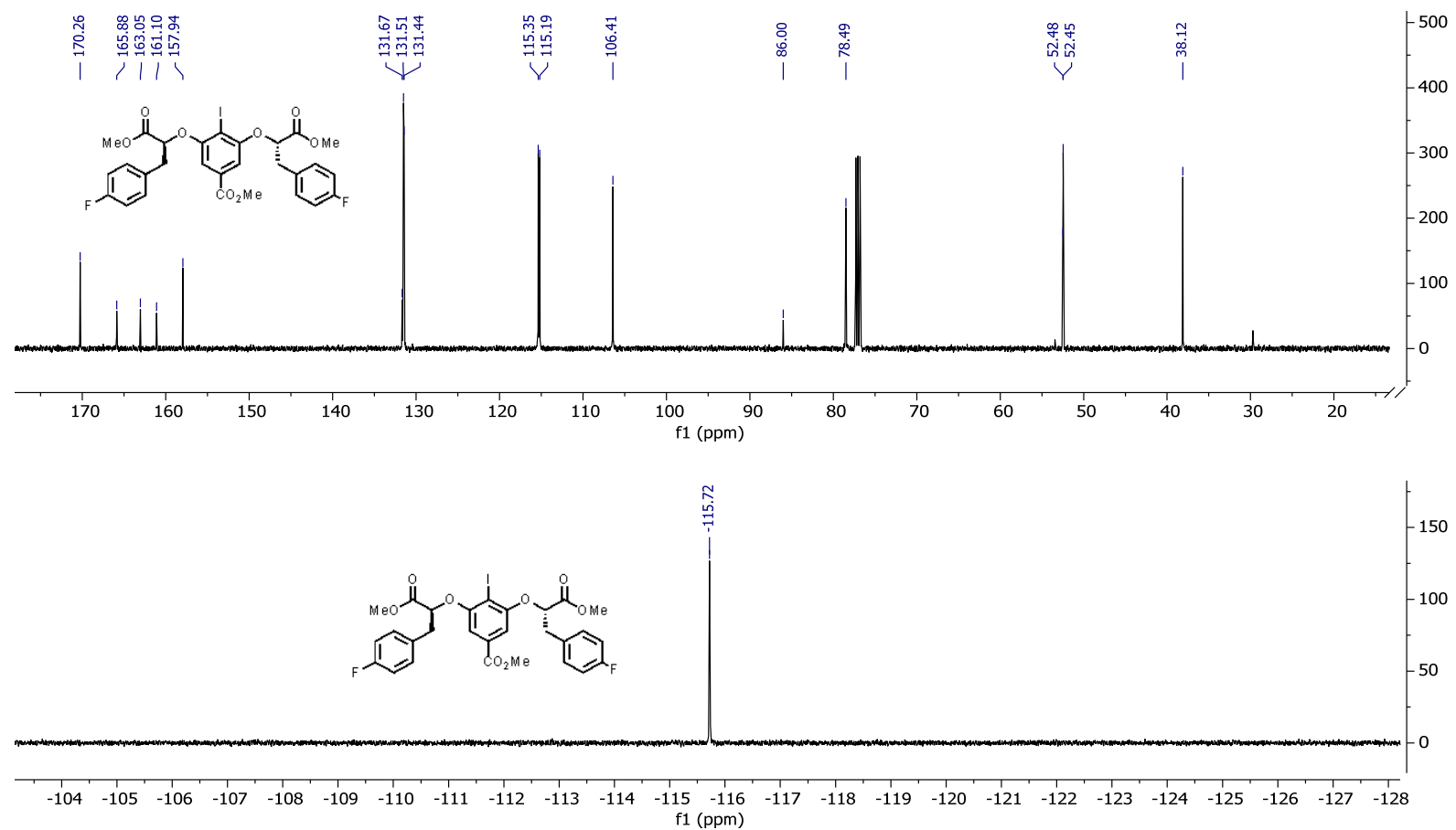<smiles>COC(=O)c1cc(O[C@@H](Cc2ccc(Cl)cc2)C(=O)OC)c(I)c(O[C@H](Cc2ccc(Cl)cc2)C(=O)OC)c1</smiles>

dimethyl 2,2'-((2-iodo-5-(methoxycarbonyl)-1,3-phenylene)bis(oxy))(2S,2'S)-bis(3-(4-chlorophenyl)propanoate). $3 \mathrm{I}$ was isolated as a crystalline, white powder.

${ }^{1} \mathrm{H}$ NMR $\left(500 \mathrm{MHz}, \mathrm{CDCl}_{3}\right) \delta 7.35(\mathrm{~d}, J=8.4 \mathrm{~Hz}, 4 \mathrm{H}), 7.26(\mathrm{~d}, J=8.0 \mathrm{~Hz}, 6 \mathrm{H}), 6.86(\mathrm{~s}, 2 \mathrm{H}), 4.90(\mathrm{t}, J=6.2 \mathrm{~Hz}$, $3 \mathrm{H}), 3.83(\mathrm{~s}, 3 \mathrm{H}), 3.69(\mathrm{~s}, 6 \mathrm{H}), 3.35-3.25(\mathrm{~m}, 4 \mathrm{H})$. 
${ }^{13} \mathrm{C}$ NMR $\left(126 \mathrm{MHz}, \mathrm{CDCl}_{3}\right) \delta 170.15,165.85,157.90,134.29,133.07,131.69,131.31,128.58,106.42,85.98$, $78.30,52.51,38.26$.

HRMS (ESI): for $\mathrm{C}_{28} \mathrm{H}_{26} \mathrm{Cl}_{2} \mathrm{IO}_{8},[\mathrm{M}+\mathrm{H}]^{+}$calculated $m / z=687.0044$, found $m / z=687.0037$.
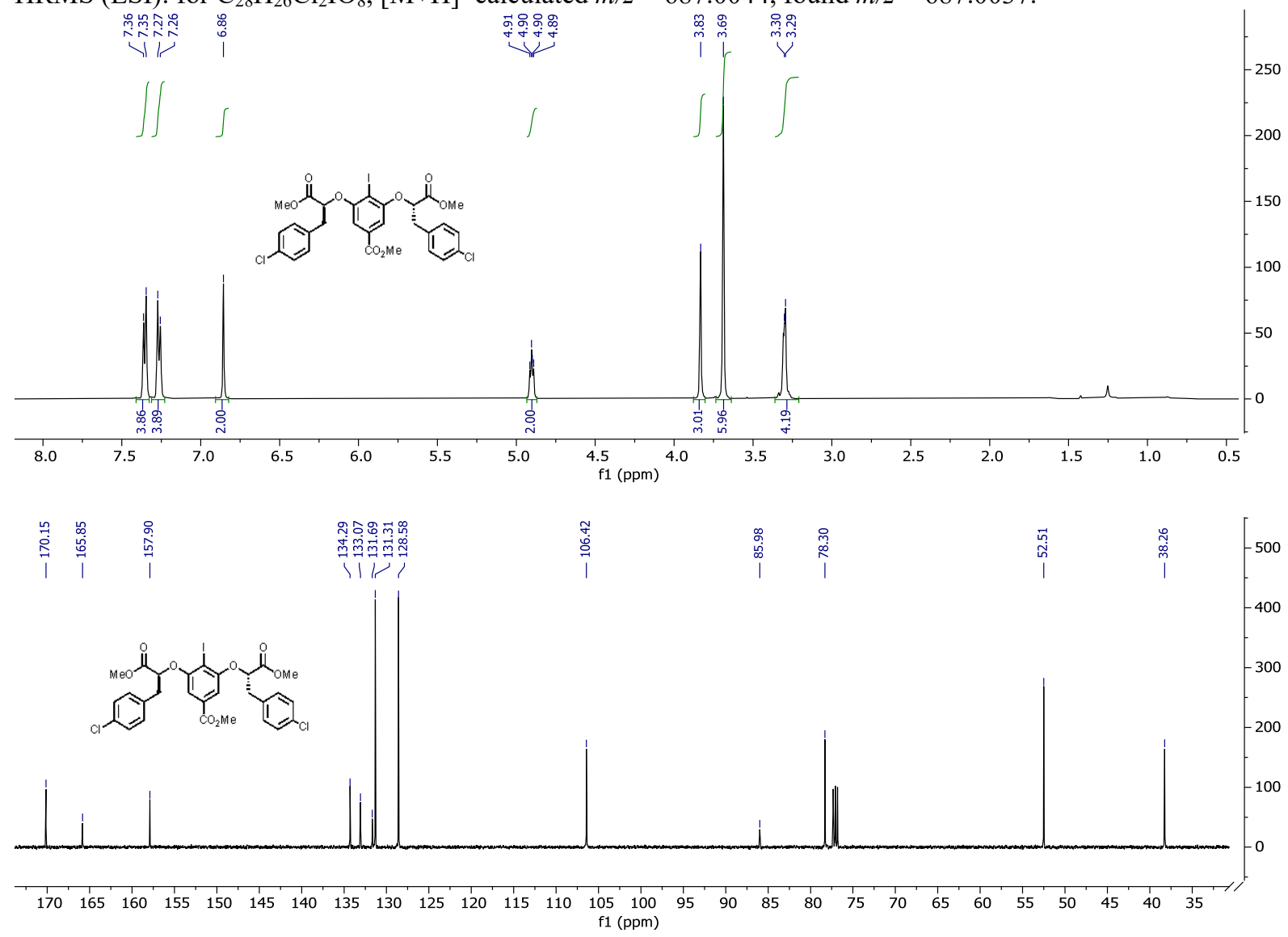<smiles>COC(=O)[C@H](Cc1cccc(Cl)c1)Oc1cc(O[C@H](Cc2cccc(Cl)c2)C(=O)OC)cc(OC(C)=O)c1I</smiles>

dimethyl 2,2'-((2-iodo-5-(methoxycarbonyl)-1,3-phenylene)bis(oxy))(2S,2'S)-bis(3-(3-chlorophenyl)propanoate). $3 \mathbf{m}$ was isolated as a crystalline, white powder.

${ }^{1} \mathrm{H}$ NMR $\left(500 \mathrm{MHz}, \mathrm{CDCl}_{3}\right) \delta 7.47$ (s, 2H), $7.29(\mathrm{~d}, J=4.7 \mathrm{~Hz}, 2 \mathrm{H}), 7.22(\mathrm{~d}, J=4.4 \mathrm{~Hz}, 4 \mathrm{H}), 6.86(\mathrm{~s}, 2 \mathrm{H}), 4.91$ (t, $J$ $=6.0 \mathrm{~Hz}, 2 \mathrm{H}), 3.84(\mathrm{~s}, 3 \mathrm{H}), 3.70(\mathrm{~s}, 6 \mathrm{H}), 3.35-3.25(\mathrm{~m}, 4 \mathrm{H})$.

${ }^{13} \mathrm{C}$ NMR $\left(126 \mathrm{MHz}, \mathrm{CDCl}_{3}\right) \delta 170.13,165.86,157.88,137.75,134.14,131.63,130.27,129.67,128.04,127.38$, $106.38,85.92,78.19,52.52,38.56$.

HRMS (ESI): for $\mathrm{C}_{28} \mathrm{H}_{26} \mathrm{Cl}_{2} \mathrm{IO}_{8},[\mathrm{M}+\mathrm{H}]^{+}$calculated $m / z=687.0044$, found $m / z=687.0040$. 

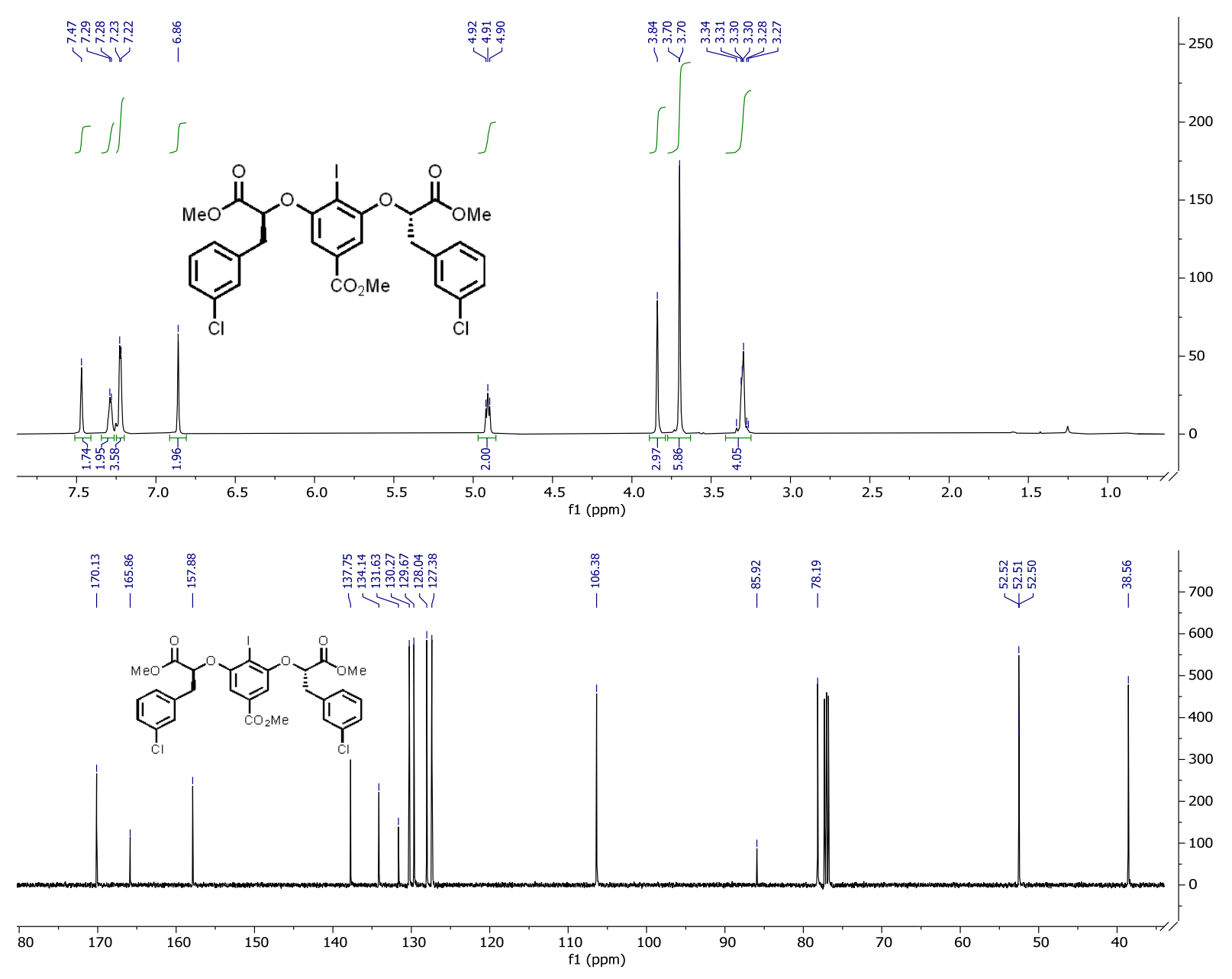<smiles>COC(=O)c1cc(O[C@@H](Cc2cccc(C)c2)C(=O)OC)c(I)c(O[C@H](Cc2cccc(C)c2)C(=O)OC)c1</smiles>

dimethyl 2,2'-((2-iodo-5-(methoxycarbonyl)-1,3-phenylene)bis(oxy))(2S,2'S)-bis(3-(m-tolyl)propanoate). 3n was isolated as a crystalline, white powder.

${ }^{1} \mathrm{H}$ NMR (500 MHz, $\left.\mathrm{CDCl}_{3}\right) \delta 7.27$ (s, 2H), $7.21-7.15(\mathrm{~m}, 4 \mathrm{H}), 7.08-7.03(\mathrm{~m}, 2 \mathrm{H}), 6.85(\mathrm{~s}, 2 \mathrm{H}), 4.89$ (dd, J= 7.9, $4.5 \mathrm{~Hz}, 2 \mathrm{H}), 3.83(\mathrm{~s}, 3 \mathrm{H}), 3.69(\mathrm{~s}, 6 \mathrm{H}), 3.34-3.23(\mathrm{~m}, 5 \mathrm{H}), 2.34(\mathrm{~s}, 6 \mathrm{H})$.

${ }^{13} \mathrm{C}$ NMR $\left(126 \mathrm{MHz}, \mathrm{CDCl}_{3}\right) \delta 170.57,165.97,158.06,137.96,135.82,131.61,130.87,128.35,127.88,126.82$, $106.33,86.01,78.79,52.47,52.41,38.98,21.41$.

HRMS (ESI): for $\mathrm{C}_{30} \mathrm{H}_{32} \mathrm{IO}_{8},[\mathrm{M}+\mathrm{H}]^{+}$calculated $\mathrm{m} / z=647.1136$, found $\mathrm{m} / z=647.1132$. 

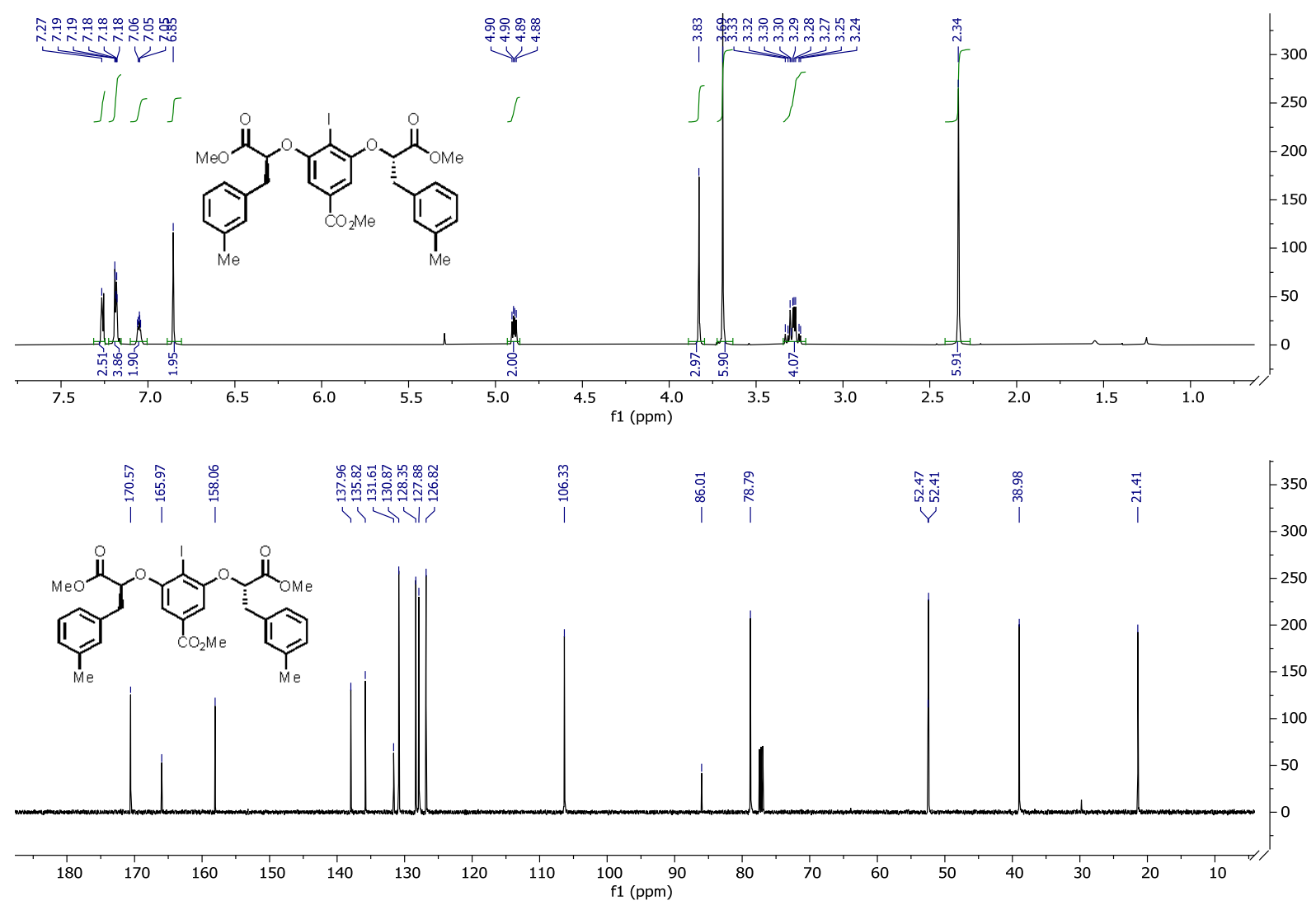<smiles>COC(=O)c1cc(O[C@@H](Cc2ccccc2C)C(=O)OC)c(I)c(O[C@H](Cc2ccccc2C)C(=O)OC)c1</smiles>

dimethyl 2,2'-((2-iodo-5-(methoxycarbonyl)-1,3-phenylene)bis(oxy))(2S,2'S)-bis(3-(o-tolyl)propanoate). 3o was isolated as a crystalline, white powder.

${ }^{1} \mathrm{H}$ NMR $\left(500 \mathrm{MHz}, \mathrm{CDCl}_{3}\right) \delta 7.41-7.36(\mathrm{~m}, 3 \mathrm{H}), 7.17-7.11(\mathrm{~m}, 7 \mathrm{H}), 6.85(\mathrm{~s}, 2 \mathrm{H}), 4.89(\mathrm{dd}, J=8.4,4.8 \mathrm{~Hz}, 3 \mathrm{H})$, $3.83(\mathrm{~s}, 3 \mathrm{H}), 3.69(\mathrm{~s}, 6 \mathrm{H}), 3.40-3.27(\mathrm{~m}, 5 \mathrm{H}), 2.43(\mathrm{~s}, 6 \mathrm{H})$.

${ }^{13} \mathrm{C}$ NMR $\left(126 \mathrm{MHz}, \mathrm{CDCl}_{3}\right) \delta 170.76,165.96,158.09,136.52,134.28,131.58,130.81,130.39,127.26,126.07$, $106.51,86.31,78.12,52.46,36.12,19.85$.

HRMS (ESI): for $\mathrm{C}_{30} \mathrm{H}_{32} \mathrm{IO}_{8},[\mathrm{M}+\mathrm{H}]^{+}$calculated $\mathrm{m} / \mathrm{z}=647.1136$, found $\mathrm{m} / \mathrm{z}=647.1132$. 

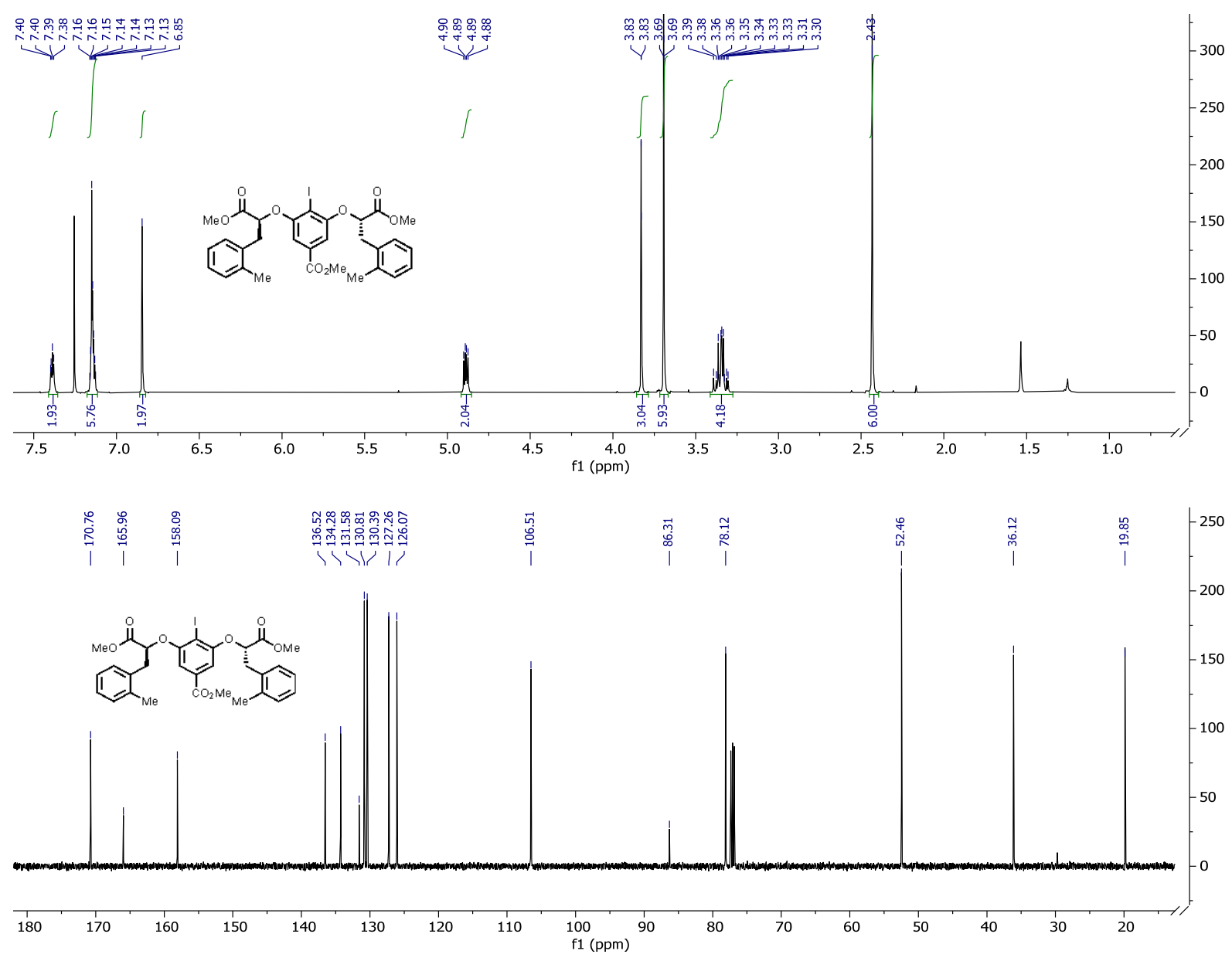<smiles>CCOC(=O)c1cc(O[C@H](Cc2ccc(C(C)(C)C)cc2)C(=O)OCc2ccc(C(C)(C)C)cc2)c(I)c(O[C@H](Cc2ccc(C(C)(C)C)cc2)C(=O)O[C@](c2cccc(C(C)(C)C)c2)([C@@H](C)Br)C(C)(C)C)c1</smiles>

methyl 3-(((S)-1-(benzyloxy)-3-(4-(tert-butyl)phenyl)-1-oxopropan-2-yl)oxy)-5-(((S)-1-((1S,2R)-2-bromo-1-fluoro1-(3-nitrophenyl)propoxy)-3-(4-(tert-butyl)phenyl)-1-oxopropan-2-yl)oxy)-4-iodobenzoate (4)

Isolated from crude reaction mixture as a minor component of the recovered catalyst, purified by repeated chromatography on $\mathrm{SiO}_{2}$ with Hexanes/Et ${ }_{2} \mathrm{O}$ and Hexanes/DCM. For characterization purposes, a modified reaction protocol was conducted to obtain larger quantities $(30 \mathrm{mg}, 28 \%$ yield):

$242 \mathrm{mg}$ substrate

$88 \mathrm{mg}$ catalyst

$250 \mathrm{mg}$ mCPBA (unpurified)

$577 \mathrm{uL}$ pyHF

$2.3 \mathrm{~mL} \mathrm{DCM}$ 
Note: Relative stereochemistry of the bromostyrene-derived side-arm was not directly determined and is assigned on the basis of the major product enantiomer.

${ }^{1} \mathrm{H}$ NMR $(500 \mathrm{MHz}$, Chloroform- $d$ ) $\delta 8.15(\mathrm{dt}, J=7.7,2.2 \mathrm{~Hz}, 1 \mathrm{H}), 7.97(\mathrm{~d}, J=2.3 \mathrm{~Hz}, 1 \mathrm{H}), 7.43(\mathrm{~d}, J=8.2 \mathrm{~Hz}$, $2 \mathrm{H}), 7.38(\mathrm{~d}, J=8.1 \mathrm{~Hz}, 2 \mathrm{H}), 7.36-7.24(\mathrm{~m}, 9 \mathrm{H}), 7.24-7.18(\mathrm{~m}, 2 \mathrm{H}), 6.95(\mathrm{~s}, 1 \mathrm{H}), 6.91(\mathrm{~s}, 1 \mathrm{H}), 5.12(\mathrm{~s}, 2 \mathrm{H}), 5.05$ $-4.98(\mathrm{~m}, 2 \mathrm{H}), 4.17(\mathrm{dq}, J=13.6,6.8 \mathrm{~Hz}, 1 \mathrm{H}), 3.80(\mathrm{~s}, 3 \mathrm{H}), 3.48-3.24(\mathrm{~m}, 4 \mathrm{H}), 1.39(\mathrm{~d}, J=5.9 \mathrm{~Hz}, 3 \mathrm{H}), 1.32(\mathrm{~s}$, 9H), 1.27 (s, 9H).

${ }^{19} \mathrm{~F}$ NMR (471 MHz, Chloroform- $d$ ) $\delta-117.34$ (broad s).

${ }^{13} \mathrm{C}$ NMR (126 MHz, Chloroform- $d$ ) $\delta 170.05,166.47,165.76,158.41,157.65,150.47,150.04,148.07,136.63$ (d, $J$ $=27.7 \mathrm{~Hz}), 135.12,132.84,132.05(\mathrm{~d}, J=7.0 \mathrm{~Hz}), 131.98,131.95,129.92,129.70,129.24,128.62,128.53,125.65$, $125.48,124.49,121.11(\mathrm{~d}, J=7.9 \mathrm{~Hz}), 111.41(\mathrm{~d}, J=242.4 \mathrm{~Hz}), 107.00,105.94,86.08,78.82,78.32,67.34,52.62$, 49.37 (d, $J=29.0 \mathrm{~Hz}), 38.51,38.34,36.77,34.65,34.57,31.51,31.48,19.57$ (d, $J=3.0 \mathrm{~Hz})$.

HRMS (ESI): for $\mathrm{C}_{50} \mathrm{H}_{53} \mathrm{BrFINO}_{10},[\mathrm{M}+\mathrm{H}]^{+}$calculated $\mathrm{m} / z=1052.1876$ and 154.1856 , found $\mathrm{m} / \mathrm{z}=1052.1869$ and 1054.1863.
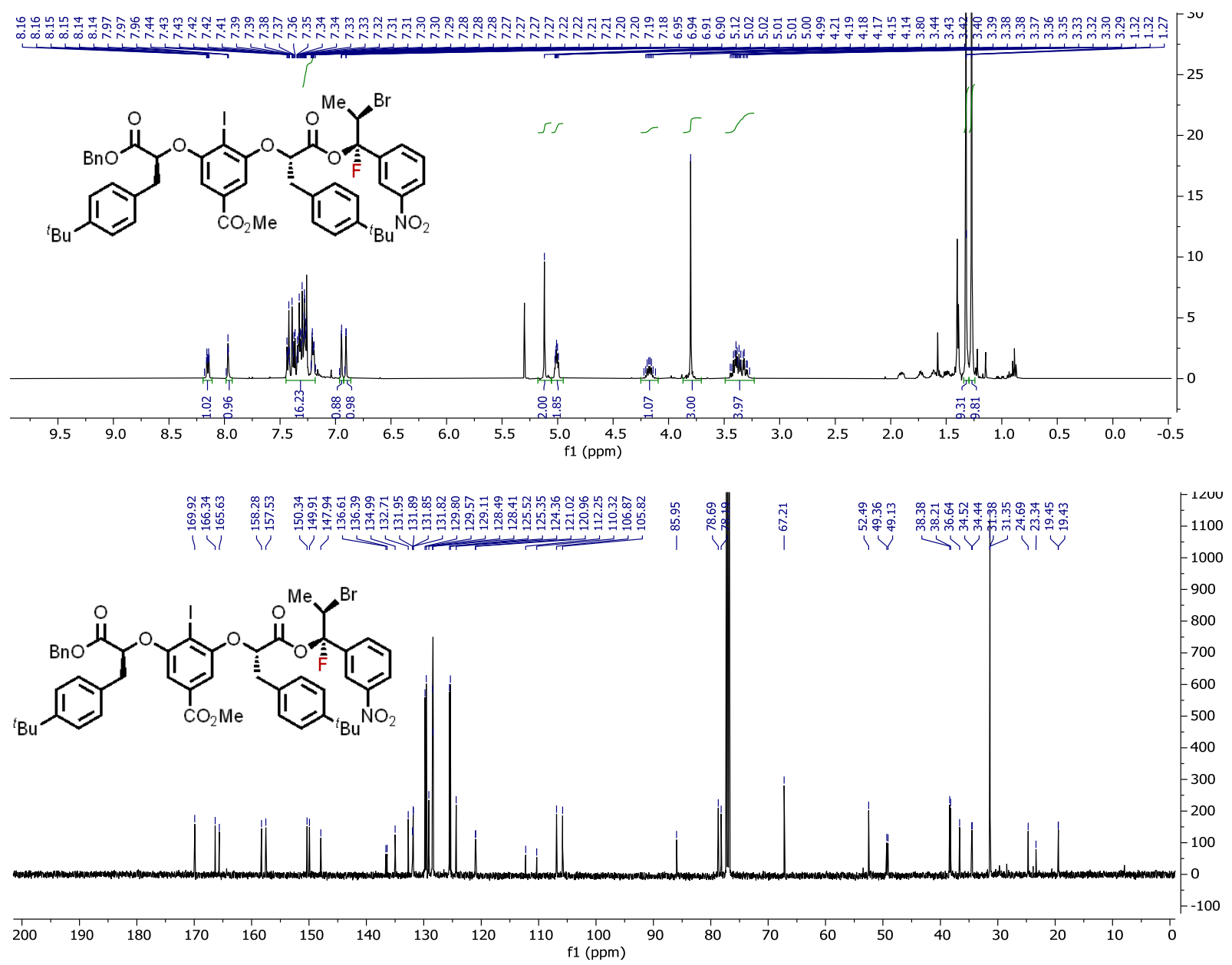


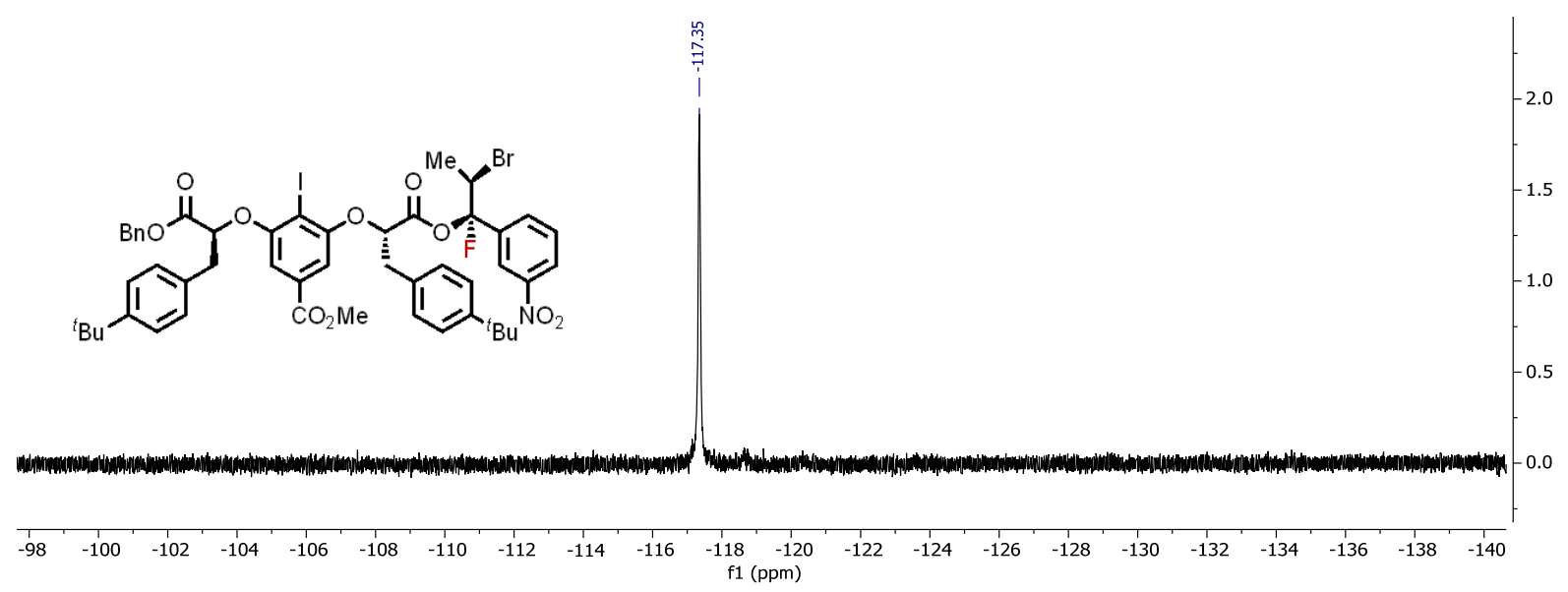

$3 a$

$3 b$

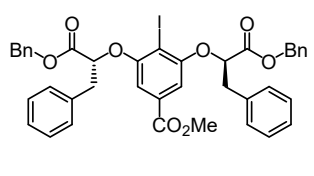

$3 e$<smiles>CCc1ccc(CC(=O)OC(Cc2ccc(CC)cc2)c2cc(OC)c(C)c(OC(C(=O)OC)c3ccc(CC)cc3)c2)cc1</smiles>

$86 \%$ ee

$3 i$

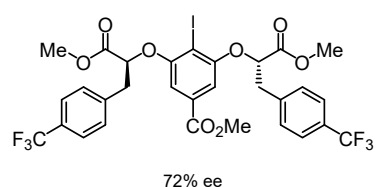

$3 j$

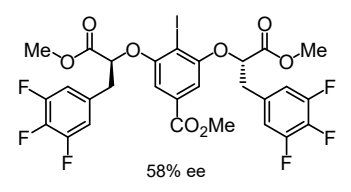

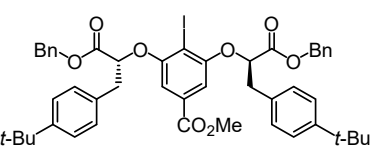

$-90 \%$ ee

$3 d$

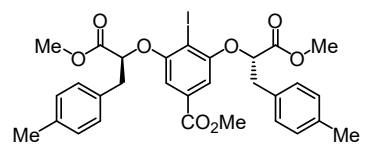

$83 \%$ ee

$3 k$

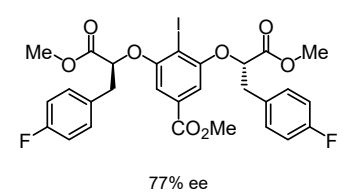

$3 h$

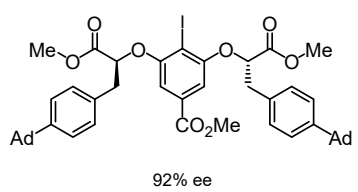

$3 g$

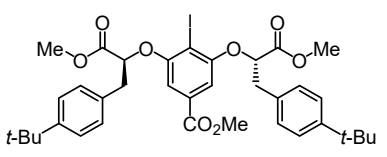

$91 \%$ ee

30

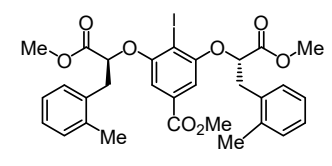

$88 \%$ ee

31
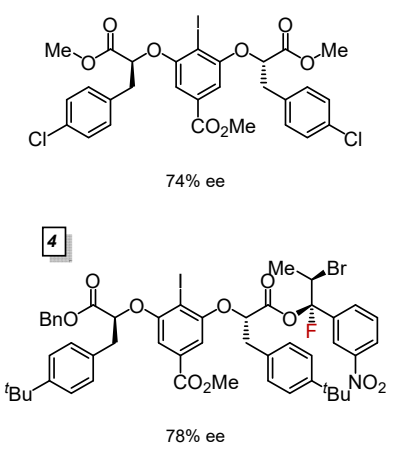

$3 f$

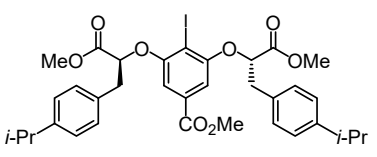

$90 \%$ ee

$3 n$

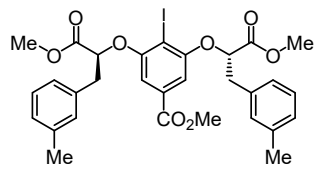

$84 \%$ ee

$3 m$

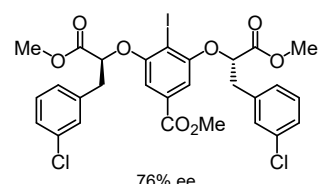

$3 a$

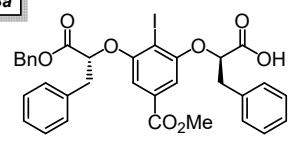

$-69 \%$ ee

Figure S1. Catalyst structure-activity relationship study. Enantiomeric excesses were obtained using substrate 2a under the standard conditions (vide infra). Methyl-ester-substituted catalysts did not undergo decomposition to iodoarenes analogous to 4 . These catalysts offered lower yields of product 1a but with comparable enantioselectivities (cf. 3b vs. 3g) and were employed in the SAR study due to their simpler preparation. 


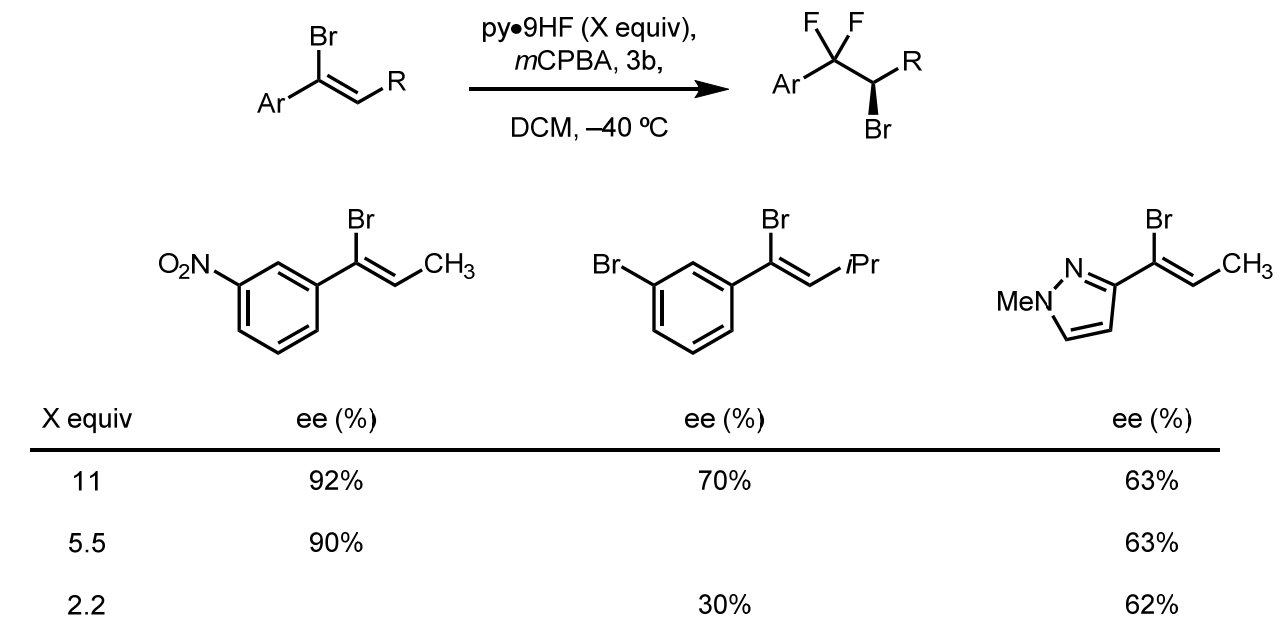

Figure S2. 9HF·Pyridine loading optimization

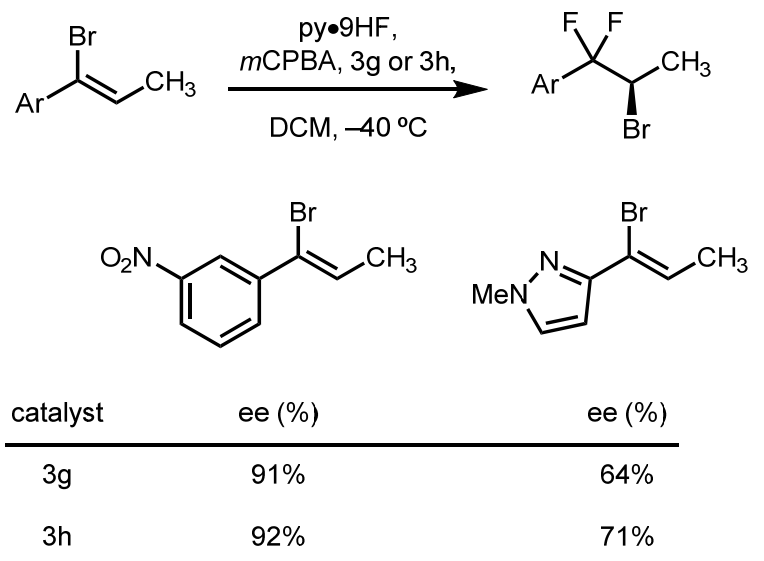

Figure S3. Para-Adamantyl Catalyst Effect 


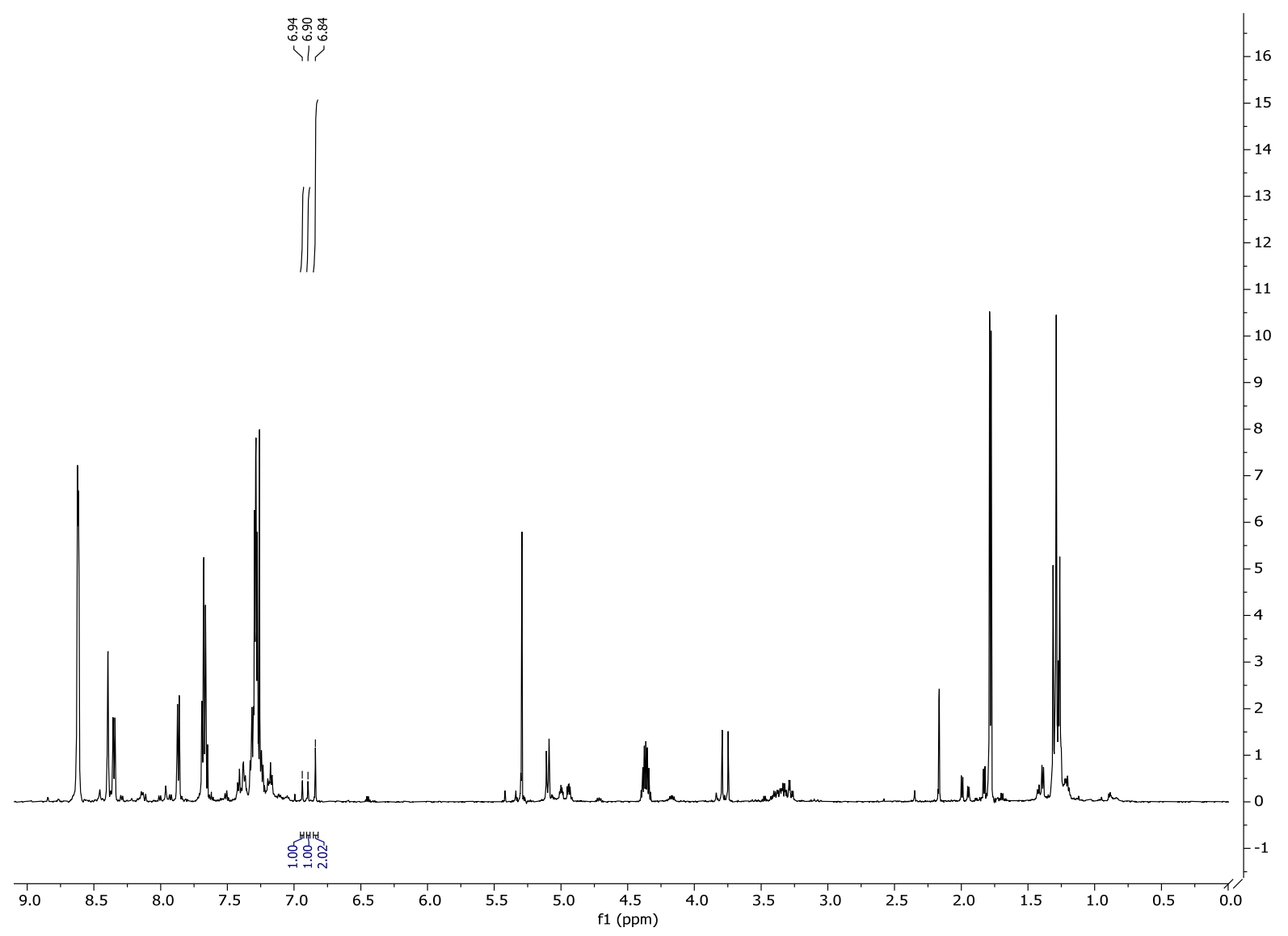

Figure S4. ${ }^{1} \mathrm{H}$ NMR spectrum of crude reaction mixture of fluorination of $\mathbf{2 a}$ catalyzed by $\mathbf{3 b}(20 \mathrm{~mol} \%)$. Catalyst decomposition product 4 resonances can be observed at $6.94 \mathrm{ppm}$ and $6.90 \mathrm{ppm}$. 


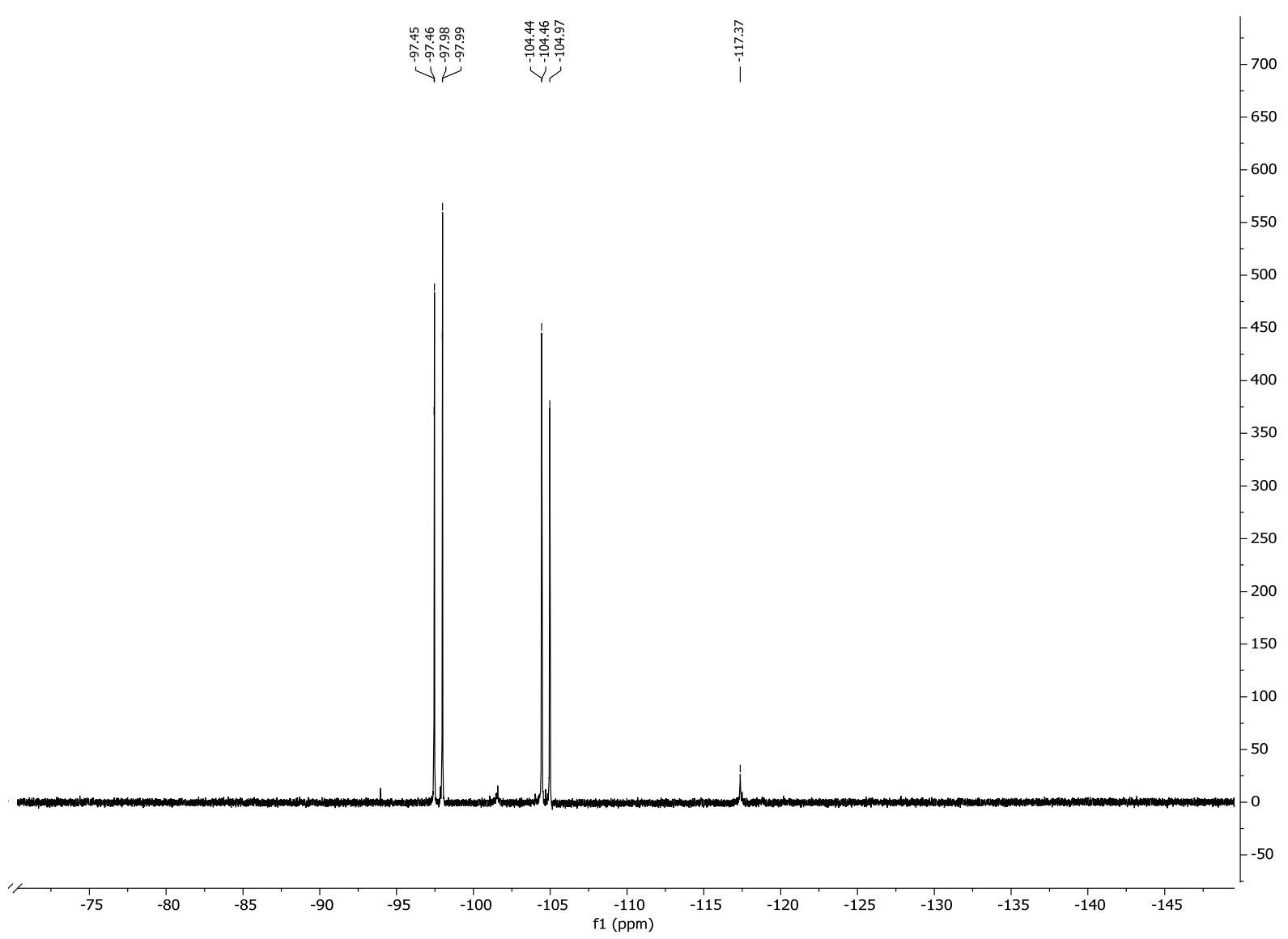

Figure S5. ${ }^{19} \mathrm{~F}$ NMR spectrum of crude reaction mixture of fluorination of $\mathbf{2 a}$ catalyzed by $\mathbf{3 b}(20 \mathrm{~mol} \%)$. Catalyst decomposition product 4 resonance can be observed at $-117.37 \mathrm{ppm}$. 


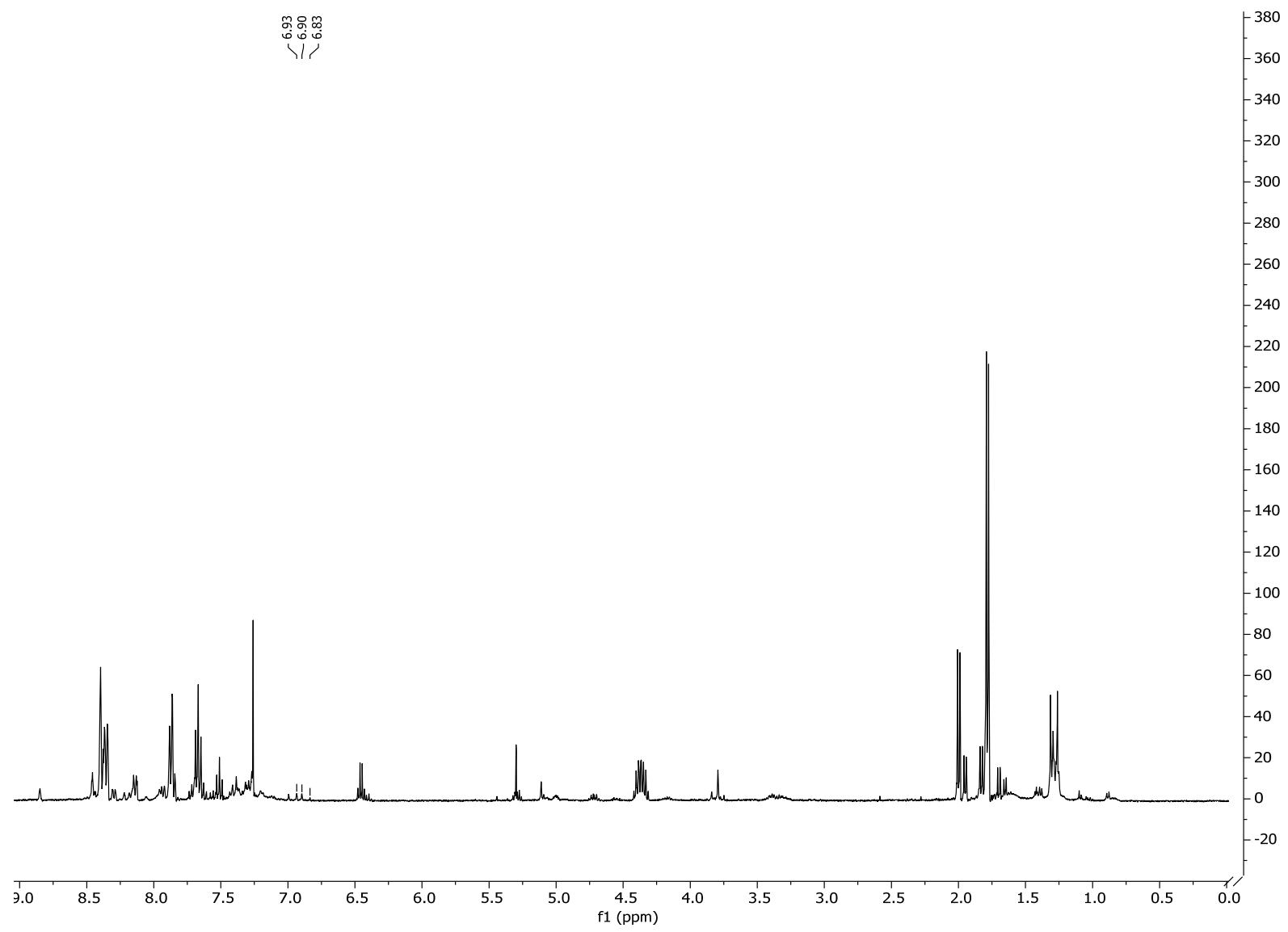

Figure S6. ${ }^{1} \mathrm{H}$ NMR spectrum of crude reaction mixture of fluorination of $\mathbf{2 a}$ catalyzed by $\mathbf{3 b}(10 \mathrm{~mol} \%)$. Catalyst decomposition product's (4) diagnostic resonances can be observed at $6.94 \mathrm{ppm}$ and $6.90 \mathrm{ppm}$. Parent catalyst's (3b) diagnostic resonance at $6.83 \mathrm{ppm}$ is barely observable. Significant starting material $(\sim 6.45 \mathrm{ppm})$ remains. 


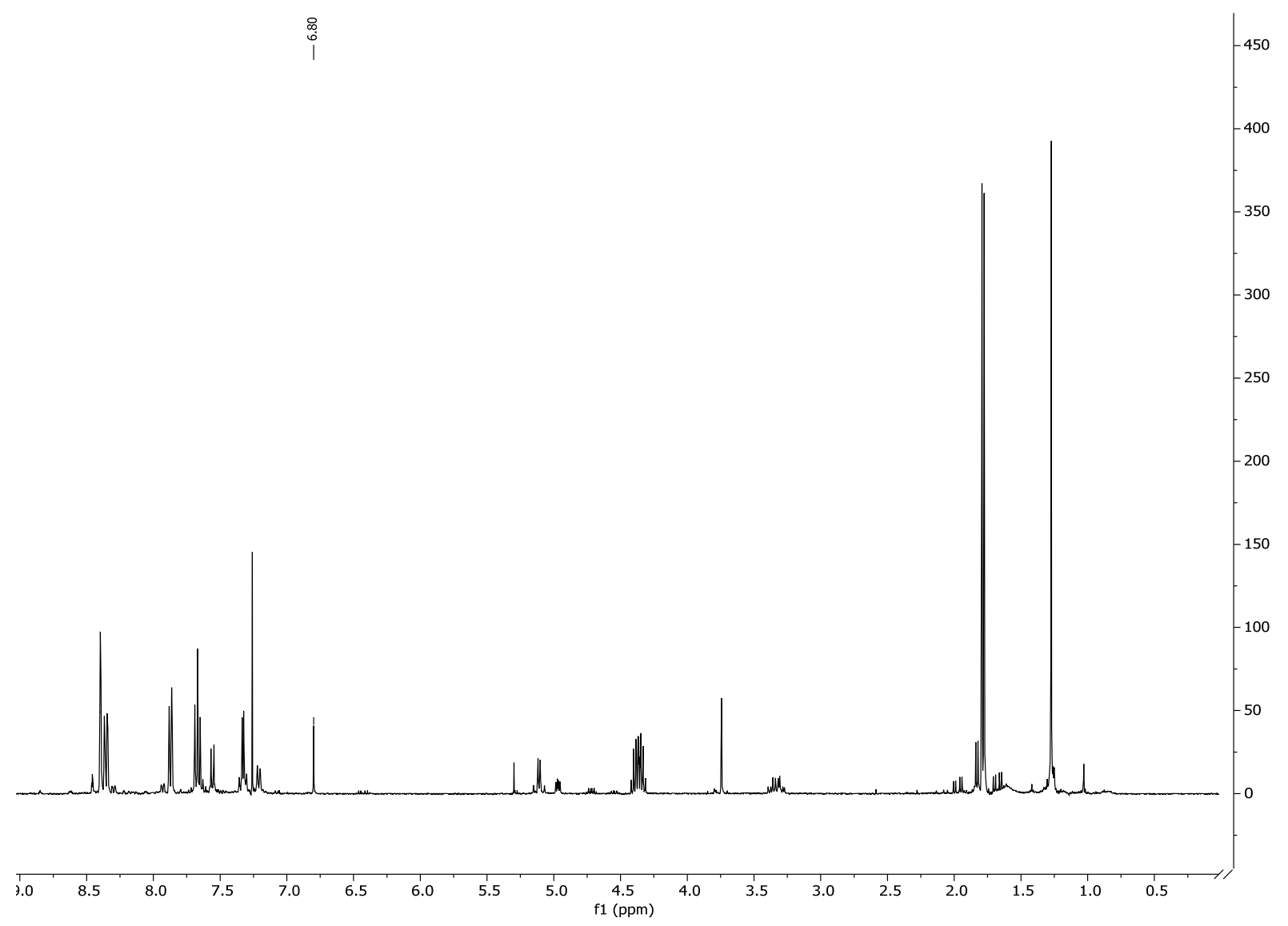

Figure S7. ${ }^{1} \mathrm{H}$ NMR spectrum of crude reaction mixture of fluorination of $2 \mathbf{a}$ catalyzed by $3 \mathbf{c}(10 \mathrm{~mol} \%)$. No catalyst decomposition products analogous to $\mathbf{4}$ are observed. 


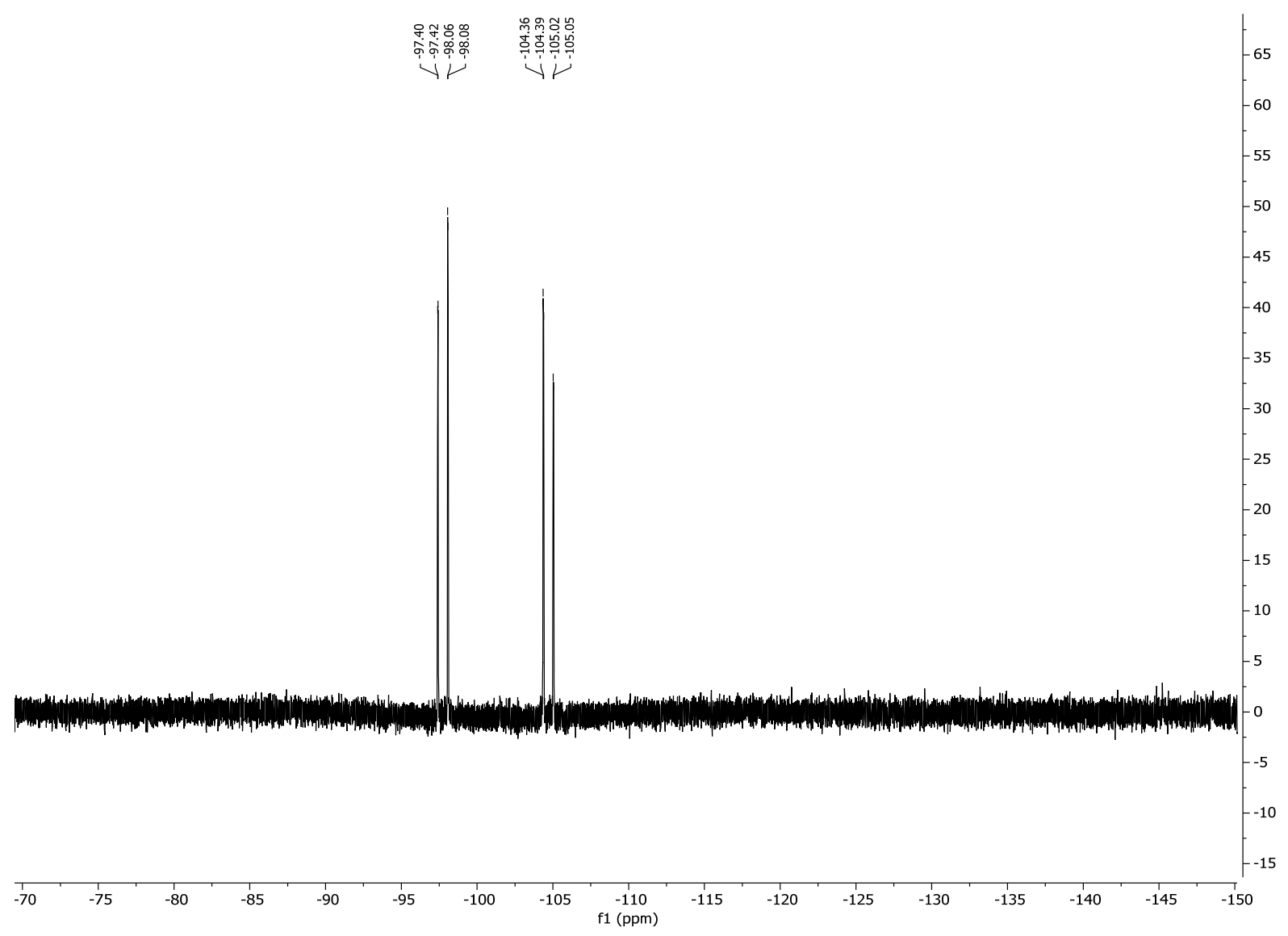

Figure S8. ${ }^{19} \mathrm{~F}$ NMR spectrum of crude reaction mixture of fluorination of $2 \mathbf{a}$ catalyzed by $\mathbf{3 c}(10 \mathrm{~mol} \%)$. No catalyst decomposition products analogous to $\mathbf{4}$ are observed. 


\section{General Procedure for Oxidative Rearrangement}<smiles>[R]C=C(Br)c1ccccc1</smiles>

To a solution of substrate ( $0.2 \mathrm{mmol}, 1$ equiv) and catalyst $3 \mathbf{c}(23 \mathrm{mg}, 0.02 \mathrm{mmol}, 10 \mathrm{~mol} \%)$ in dichloromethane $(2.3$ $\mathrm{mL}$ ) in a low-density polyethylene tube at $-78{ }^{\circ} \mathrm{C}$ was added $\mathrm{HF} \cdot$ Pyridine (py $9 \mathrm{HF}, 70 \%$ hydrogen fluoride by weight, $577 \mu \mathrm{L}, 100$ equiv hydrogen fluoride) followed by $m \mathrm{CPBA}^{6}(41.4 \mathrm{mg}, 0.24 \mathrm{mmol}, 1.2$ equiv). The reaction was warmed to $-40^{\circ} \mathrm{C}$ and stirred at that temperature for 48 hours. The heterogeneous mixture was then cooled to $-78^{\circ} \mathrm{C}$ and transferred carefully into a vigorously stirred suspension of basic alumina $(2.0 \mathrm{~g})$ in dichloromethane at $-78^{\circ} \mathrm{C}$. The resulting suspension was allowed to warm to room temperature and was filtered through addition basic alumina, washing with $10 \mathrm{~mL}$ dichloromethane. The combined filtrate was concentrated in vacuo and purified by column chromatography.

Racemic samples were prepared using the same procedure with the following modification: Iodobenzene or iodotoluene (20 mol\%) were used in place of $\mathbf{3 c}$.<smiles>CC(Br)C(F)(F)c1cccc([N+](=O)[O-])c1</smiles>

(R)-1-(2-bromo-1,1-difluoropropyl)-3-nitrobenzene. 1a was prepared from $2 \mathrm{a}$ (48.4 $\mathrm{mg}, 0.2 \mathrm{mmol}$ ) according to the General Procedure as a clear, colorless oil $(47.1 \mathrm{mg}, 84 \%$ yield $)$.

${ }^{1} \mathrm{H}$ NMR $\left(500 \mathrm{MHz}, \mathrm{CDCl}_{3}\right) \delta 8.40(\mathrm{~s}, 1 \mathrm{H}), 8.36(\mathrm{~d}, J=8.5 \mathrm{~Hz}, 1 \mathrm{H}), 7.87(\mathrm{~d}, J=8.5 \mathrm{~Hz}, 1 \mathrm{H}), 7.67(\mathrm{t}, J=8.0 \mathrm{~Hz}$, $1 \mathrm{H}), 4.47-4.27(\mathrm{~m}, 1 \mathrm{H}), 1.79(\mathrm{~d}, J=6.9 \mathrm{~Hz}, 3 \mathrm{H})$.

${ }^{13} \mathrm{C}$ NMR $\left(126 \mathrm{MHz}, \mathrm{CDCl}_{3}\right) \delta 148.04,136.14(\mathrm{t}, J=27.8 \mathrm{~Hz}), 132.18(\mathrm{t}, J=5.9 \mathrm{~Hz}), 129.58,125.31,121.54(\mathrm{t}, J=$ $6.5 \mathrm{~Hz}), 119.31(\mathrm{dd}, J=248.1,247.1 \mathrm{~Hz}), 47.33(\mathrm{t}, J=32.9 \mathrm{~Hz}), 19.19(\mathrm{t}, J=3.1 \mathrm{~Hz})$.

${ }^{19} \mathrm{~F}$ NMR $\left(471 \mathrm{MHz}, \mathrm{CDCl}_{3}\right) \delta-97.75(\mathrm{~d}, J=248.0 \mathrm{~Hz}, 1 \mathrm{~F}),-104.67(\mathrm{~d}, J=247.7 \mathrm{~Hz}, 1 \mathrm{~F})$.

HRMS (EI): for $\mathrm{C}_{9} \mathrm{H}_{8} \mathrm{BrF}_{2} \mathrm{NO}_{2}$, [M] $]^{+}$calculated $\mathrm{m} / z=278.9701$ and 280.9681 , found $\mathrm{m} / z=278.9700$ and 280.9679

Chiral HPLC: Chiralpak AS-H, 1.2\% isopropanol/hexanes, $0.5 \mathrm{ml} / \mathrm{min} ; 92 \%$ ee

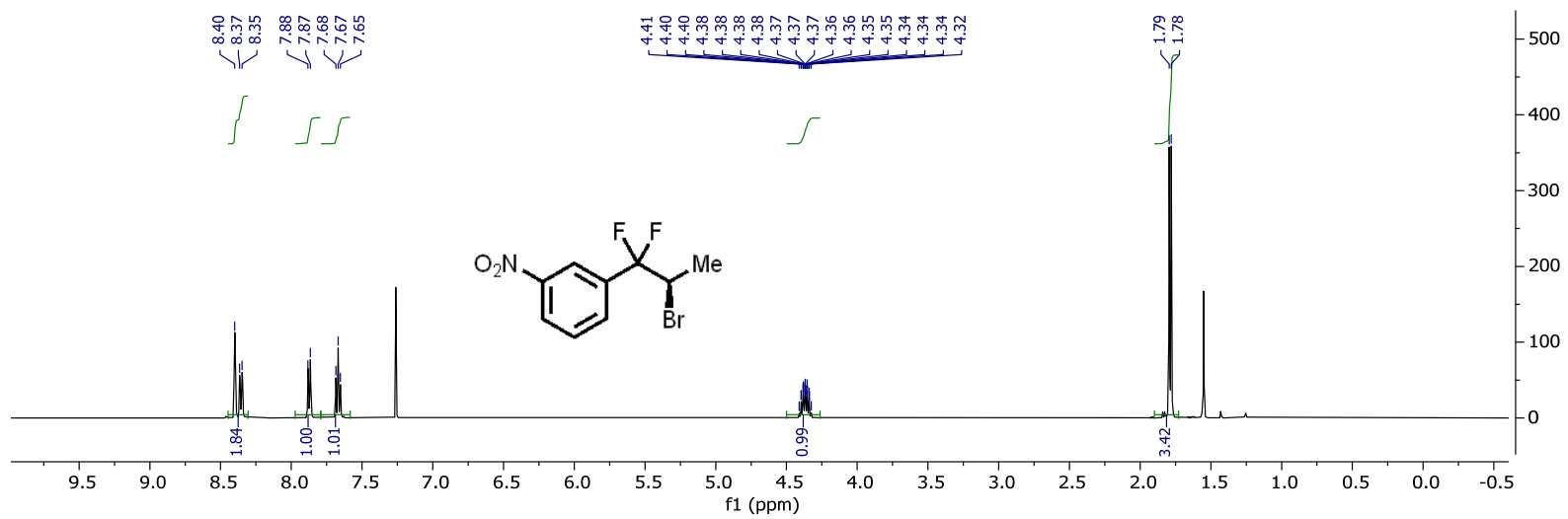



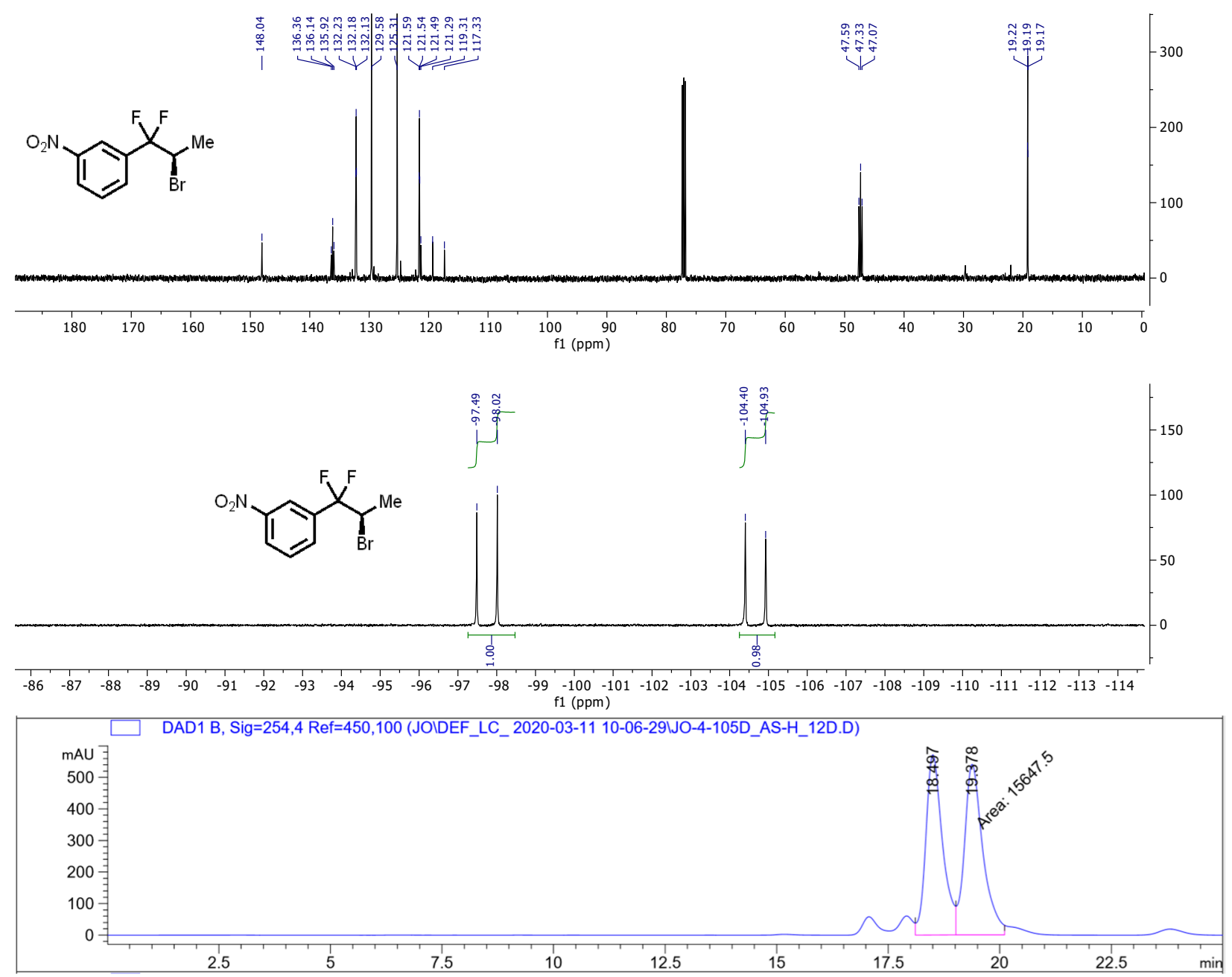

Signal 2: DAD1 B, Sig=254,4 $\operatorname{Ref}=450,100$

\begin{tabular}{|c|c|c|c|c|c|c|}
\hline $\begin{array}{c}\text { Peak } \\
\#\end{array}$ & $\begin{array}{c}\text { RetTime } \\
\text { [min] }\end{array}$ & Type & $\begin{array}{l}\text { Width } \\
\text { [min] }\end{array}$ & $\begin{array}{c}\text { Area } \\
{\left[\mathrm{mAU}^{*} \mathrm{~s}\right]}\end{array}$ & $\begin{array}{l}\text { Height } \\
{[\mathrm{mAU}]}\end{array}$ & $\begin{array}{c}\text { Area } \\
\frac{\circ}{0}\end{array}$ \\
\hline 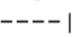 & ------ & & & -------- & ---------- & --ー-ー-ー \\
\hline 1 & 18.497 & $\mathrm{~V}$ & 6 & $1.49371 \mathrm{e} 4$ & 572.08466 & 385 \\
\hline 2 & 19.378 & $M F$ & 14 & $1.56475 \mathrm{e} 4$ & 541.74280 & 1615 \\
\hline
\end{tabular}

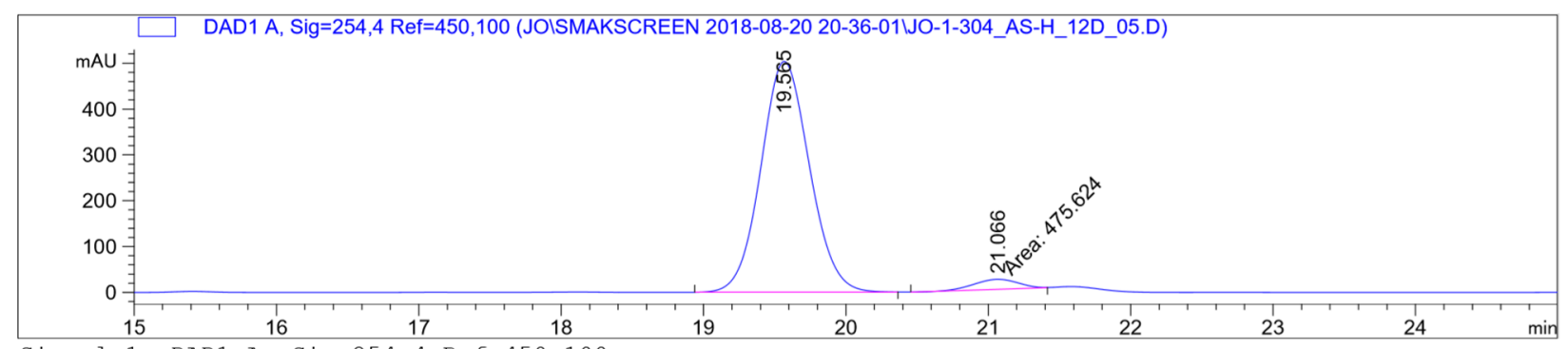

$$
\begin{aligned}
& \text { Signal 1: DAD1 A, Sig=254, } 4 \text { Ref=450,100 }
\end{aligned}
$$

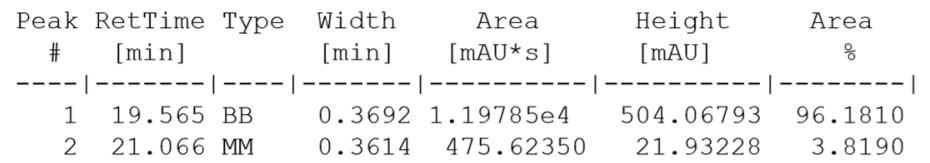


<smiles>CC(Br)C(F)(F)c1cccc(O)c1</smiles>

(R)-3-(2-bromo-1,1-difluoropropyl)phenyl trifluoromethanesulfonate. $\mathbf{1 b}$ was prepared from $\mathbf{2 b}(69.0 \mathrm{mg}, 0.2 \mathrm{mmol})$ according to the General Procedure as a clear, colorless oil $(62.1 \mathrm{mg}, 81 \%$ yield).

${ }^{1} \mathrm{H}$ NMR $\left(500 \mathrm{MHz}, \mathrm{CDCl}_{3}\right) \delta 7.58-7.55(\mathrm{~m}, 2 \mathrm{H}), 7.46-7.43(\mathrm{~m}, 1 \mathrm{H}), 7.43-7.38(\mathrm{~m}, 1 \mathrm{H}), 4.37-4.27(\mathrm{~m}, 1 \mathrm{H})$, $1.74(\mathrm{~d}, J=6.9 \mathrm{~Hz}, 3 \mathrm{H})$.

${ }^{13} \mathrm{C}$ NMR $\left(126 \mathrm{MHz}, \mathrm{CDCl}_{3}\right) \delta 149.20,136.80(\mathrm{t}, J=27.7 \mathrm{~Hz}), 130.35,126.16(\mathrm{t}, J=6.0 \mathrm{~Hz}), 123.39,119.75(\mathrm{t}, J=$ $6.5 \mathrm{~Hz}), 119.22(\mathrm{t}, J=249.7 \mathrm{~Hz}), 118.71(\mathrm{q}, J=321.3 \mathrm{~Hz}) 47.44(\mathrm{t}, J=32.8 \mathrm{~Hz}), 19.28(\mathrm{t}, J=3.1 \mathrm{~Hz})$.

${ }^{19} \mathrm{~F}$ NMR $\left(471 \mathrm{MHz}, \mathrm{CDCl}_{3}\right) \delta-72.69(\mathrm{~s}, 3 \mathrm{~F}),-98.94(\mathrm{~d}, J=247.2 \mathrm{~Hz}, 1 \mathrm{~F}),-103.19(\mathrm{~d}, J=247.0 \mathrm{~Hz}, 1 \mathrm{~F})$.

HRMS (EI): for $\mathrm{C}_{10} \mathrm{H}_{8} \mathrm{BrF}_{5} \mathrm{O}_{3} \mathrm{~S}$, [M] $]^{+}$calculated $\mathrm{m} / \mathrm{z}=381.9292$ and 383.9272 , found $\mathrm{m} / \mathrm{z}=381.9292$ and 383.9270 Chiral GC: CP-Chirasil-Dex CB, isothermal, 14 psi, $79 \%$ ee
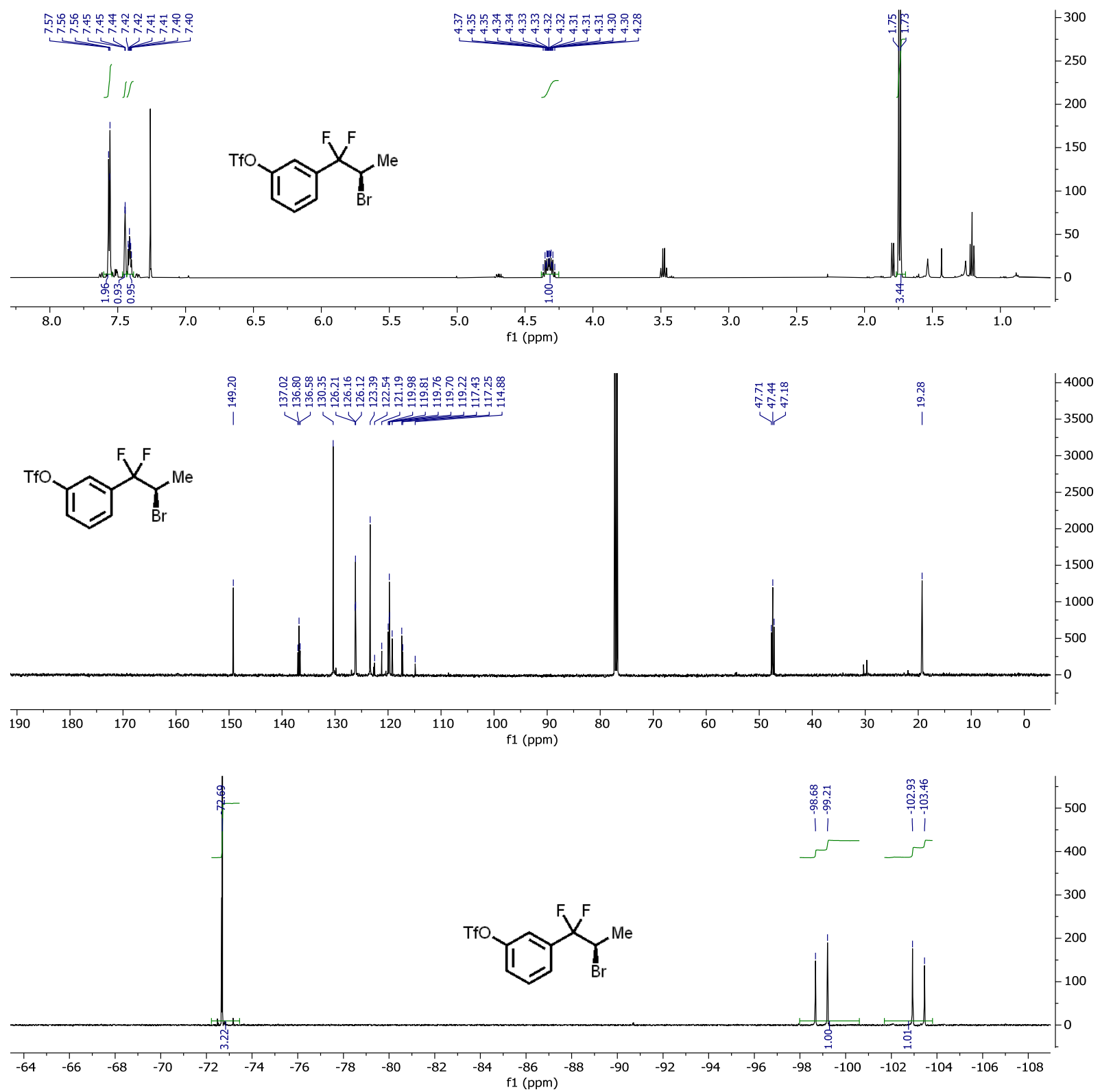

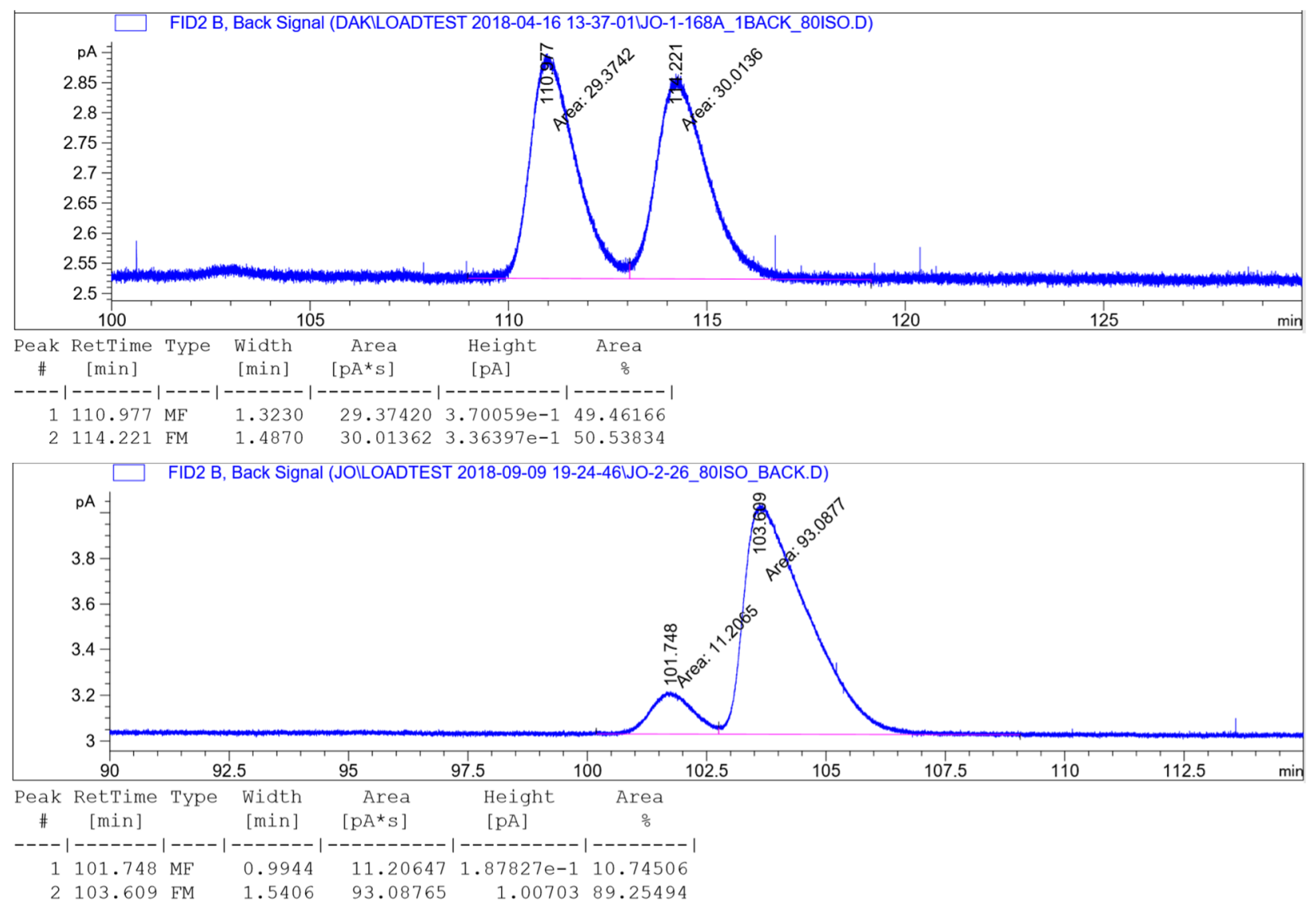<smiles>CCOC(=O)c1cccc(C(F)(F)C(Br)CC)c1</smiles>

(R)-ethyl 3-(2-bromo-1,1-difluorobutyl)benzoate. 1c was prepared from $2 \mathbf{c}(56.6 \mathrm{mg}, 0.2 \mathrm{mmol})$ according to the General Procedure as a clear, colorless oil ( $50.1 \mathrm{mg}, 78 \%$ yield).

${ }^{1} \mathrm{H}$ NMR $\left(500 \mathrm{MHz}, \mathrm{CDCl}_{3}\right) \delta 8.20-8.16(\mathrm{~m}, 1 \mathrm{H}), 8.15(\mathrm{dt}, J=7.5,1.3 \mathrm{~Hz}, 1 \mathrm{H}), 7.74-7.68(\mathrm{~m}, 1 \mathrm{H}), 7.53(\mathrm{ddt}, J=$ 7.8, 7.0, $0.8 \mathrm{~Hz}, 1 \mathrm{H}), 4.41(\mathrm{q}, J=7.2 \mathrm{~Hz}, 2 \mathrm{H}), 4.17(\mathrm{qd}, J=11.0,2.8 \mathrm{~Hz}, 1 \mathrm{H}), 2.12-2.02(\mathrm{~m}, 1 \mathrm{H}), 1.82-1.69(\mathrm{~m}$, $1 \mathrm{H}), 1.42(\mathrm{td}, J=7.1,0.8 \mathrm{~Hz}, 3 \mathrm{H}), 1.14-1.05(\mathrm{~m}, 3 \mathrm{H})$.

${ }^{13} \mathrm{C}$ NMR $\left(126 \mathrm{MHz}, \mathrm{CDCl}_{3}\right) \delta 165.72,135.04(\mathrm{t}, J=26.9 \mathrm{~Hz}), 131.36,130.84,130.34(\mathrm{t}, J=6.0 \mathrm{~Hz}), 128.43$, $127.08(\mathrm{t}, J=6.3 \mathrm{~Hz}), 119.81(\mathrm{t}, J=247.5 \mathrm{~Hz}), 61.39,57.28(\mathrm{t}, J=31.2 \mathrm{~Hz}), 25.23,14.31,12.21$.

${ }^{19} \mathrm{~F}$ NMR $\left(471 \mathrm{MHz}, \mathrm{CDCl}_{3}\right) \delta-98.58(\mathrm{~d}, J=246.1 \mathrm{~Hz}, 1 \mathrm{~F}),-100.55(\mathrm{~d}, J=246.8 \mathrm{~Hz}, 1 \mathrm{~F})$.

HRMS (ESI): for $\mathrm{C}_{13} \mathrm{H}_{16} \mathrm{BrF}_{2} \mathrm{O}_{2},[\mathrm{M}+\mathrm{H}]^{+}$calculated $\mathrm{m} / z=321.0296$ and 323.0276, found $\mathrm{m} / z=321.0297$ and 323.0276 .

Chiral HPLC: Chiralpak IB, $0.3 \%$ isopropanol/hexanes, $1.0 \mathrm{ml} / \mathrm{min}, 89 \%$ ee 

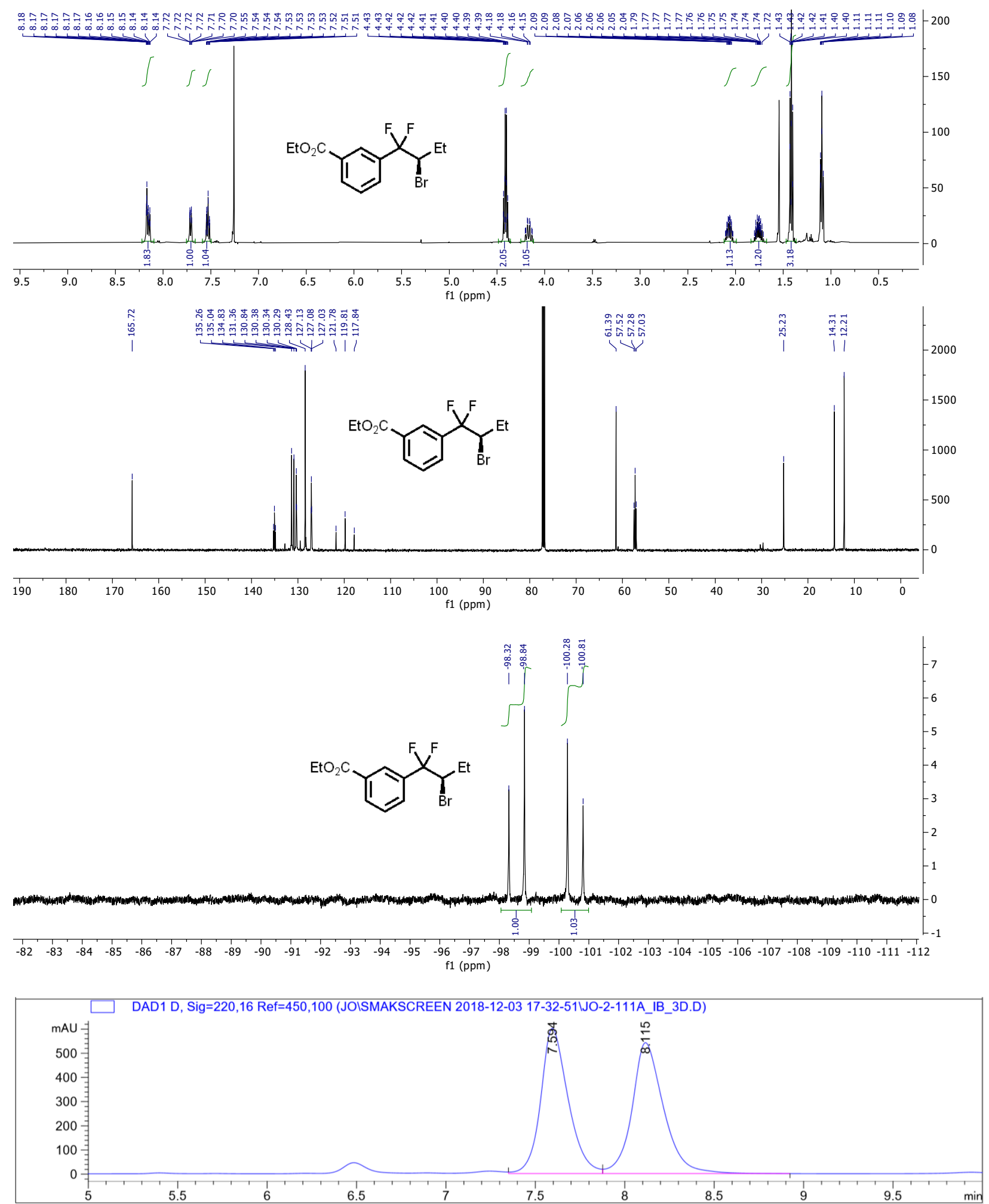

Signal 4: DAD1 D, Sig=220,16 Ref=450,100

\begin{tabular}{|c|c|c|c|c|c|c|}
\hline $\begin{array}{c}\text { Peak } \\
\#\end{array}$ & $\begin{array}{c}\text { RetTime } \\
\text { [min] }\end{array}$ & Type & $\begin{array}{l}\text { Width } \\
\text { [min] }\end{array}$ & $\begin{array}{c}\text { Area } \\
{\left[\mathrm{mAU}^{*} \mathrm{~s}\right]}\end{array}$ & $\begin{array}{l}\text { Height } \\
{[\mathrm{mAU}]}\end{array}$ & $\begin{array}{c}\text { Area } \\
\frac{\circ}{0}\end{array}$ \\
\hline & & & & -------- & & -1 \\
\hline 1 & & VV & & 6404. & 60 & 55 \\
\hline 2 & 8.115 & VB & 13 & 6656.68994 & 542.09308 & 50.9645 \\
\hline
\end{tabular}




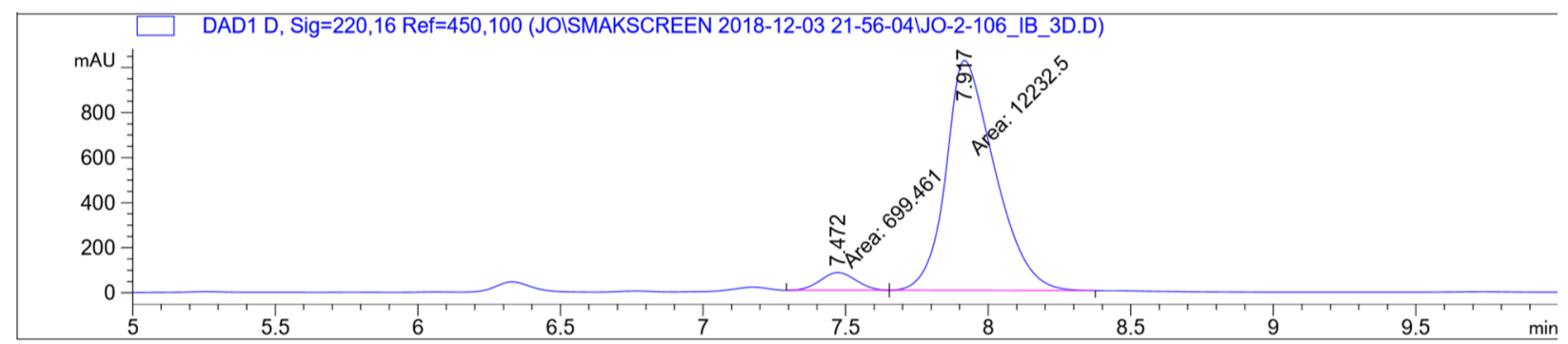

Signal 4: DAD1 D, Sig=220,16 $\operatorname{Ref}=450,100$

\begin{tabular}{|c|c|c|c|c|c|c|}
\hline $\begin{array}{c}\text { Peak } \\
\#\end{array}$ & $\begin{array}{l}\text { RetTime } \\
\text { [min] }\end{array}$ & Type & $\begin{array}{l}\text { Width } \\
\text { [min] }\end{array}$ & $\begin{array}{c}\text { Area } \\
{\left[\mathrm{mAU}^{*} \mathrm{~S}\right]}\end{array}$ & $\begin{array}{l}\text { Height } \\
{[\mathrm{mAU}]}\end{array}$ & $\begin{array}{c}\text { Area } \\
\frac{\circ}{0}\end{array}$ \\
\hline$-1-$ & - & & --- & --- & -------- & --- \\
\hline 1 & 7.472 & MF & 0.1461 & 699.46063 & 79.77899 & 088 \\
\hline 2 & 7.917 & FM & 0.1993 & $1.22325 \mathrm{e} 4$ & 1023.16272 & 94.5912 \\
\hline
\end{tabular}<smiles>CCOC(=O)c1cccc(C(F)(F)C(Br)CC(C)(C)C)c1</smiles>

(R)-ethyl 3-(2-bromo-1,1-difluorohexyl)benzoate. 1d was prepared from $\mathbf{2 d}(62.2 \mathrm{mg}, 0.2 \mathrm{mmol})$ according to the General Procedure as a clear, colorless oil ( $58.7 \mathrm{mg}, 84 \%$ yield).

${ }^{1} \mathrm{H}$ NMR $\left(500 \mathrm{MHz}, \mathrm{CDCl}_{3}\right) \delta 8.17(\mathrm{~s}, 1 \mathrm{H}), 8.15(\mathrm{~d}, J=7.9 \mathrm{~Hz}, 2 \mathrm{H}), 7.71(\mathrm{~d}, J=7.7 \mathrm{~Hz}, 1 \mathrm{H}), 7.53(\mathrm{t}, J=7.8 \mathrm{~Hz}$, $2 \mathrm{H}), 4.41(\mathrm{q}, J=7.1 \mathrm{~Hz}, 3 \mathrm{H}), 4.22(\mathrm{qd}, J=11.2,2.8 \mathrm{~Hz}, 2 \mathrm{H}), 2.04-1.93(\mathrm{~m}, 1 \mathrm{H}), 1.76$ (dddd, $J=14.2,11.0,9.5$, $4.5 \mathrm{~Hz}, 1 \mathrm{H}), 1.62(\mathrm{tt}, J=9.9,5.4 \mathrm{~Hz}, 1 \mathrm{H}), 1.42(\mathrm{t}, J=7.1 \mathrm{~Hz}, 3 \mathrm{H}), 1.39-1.21(\mathrm{~m}, 4 \mathrm{H}), 0.89(\mathrm{t}, J=7.2 \mathrm{~Hz}, 3 \mathrm{H})$. ${ }^{13} \mathrm{C}$ NMR $\left(126 \mathrm{MHz}, \mathrm{CDCl}_{3}\right) \delta 165.73,135.06(\mathrm{t}, J=26.9 \mathrm{~Hz}), 131.35,130.83,130.36(\mathrm{t}, J=6.0 \mathrm{~Hz}), 128.42$, $127.10(\mathrm{t}, J=6.3 \mathrm{~Hz}), 119.86(\mathrm{t}, J=247.6 \mathrm{~Hz}), 61.38,55.31(\mathrm{t}, J=31.4 \mathrm{~Hz}), 31.30,29.43,21.83,14.31,13.79$. ${ }^{19} \mathrm{~F}$ NMR $\left(471 \mathrm{MHz}, \mathrm{CDCl}_{3}\right) \delta-97.93(\mathrm{~d}, J=246.3 \mathrm{~Hz}, 1 \mathrm{~F}),-100.49(\mathrm{~d}, J=246.2 \mathrm{~Hz}, 1 \mathrm{~F})$.

HRMS (ESI): for $\mathrm{C}_{15} \mathrm{H}_{20} \mathrm{BrF}_{2} \mathrm{O}_{2},[\mathrm{M}+\mathrm{H}]^{+}$calculated $\mathrm{m} / z=349.0609$ and 351.0589, found $\mathrm{m} / z=349.0608$ and 351.0587.

Chiral HPLC: Chiralpak IB, $0.8 \%$ isopropanol/hexanes, $1.0 \mathrm{ml} / \mathrm{min}, 82 \%$ ee

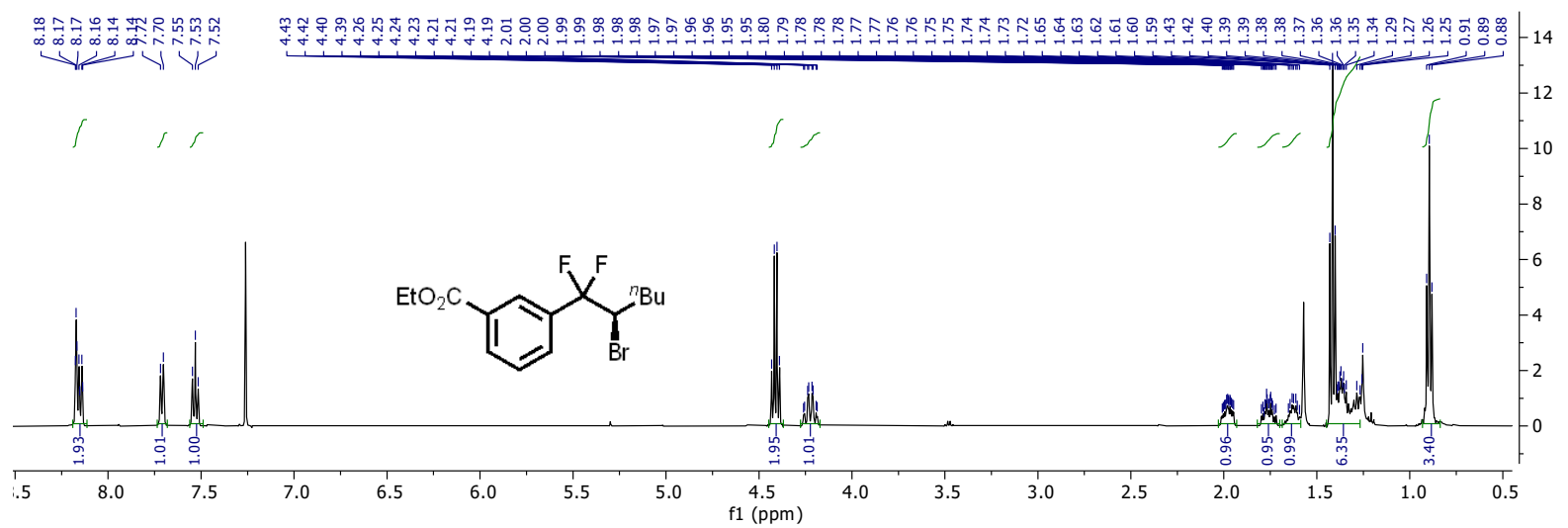



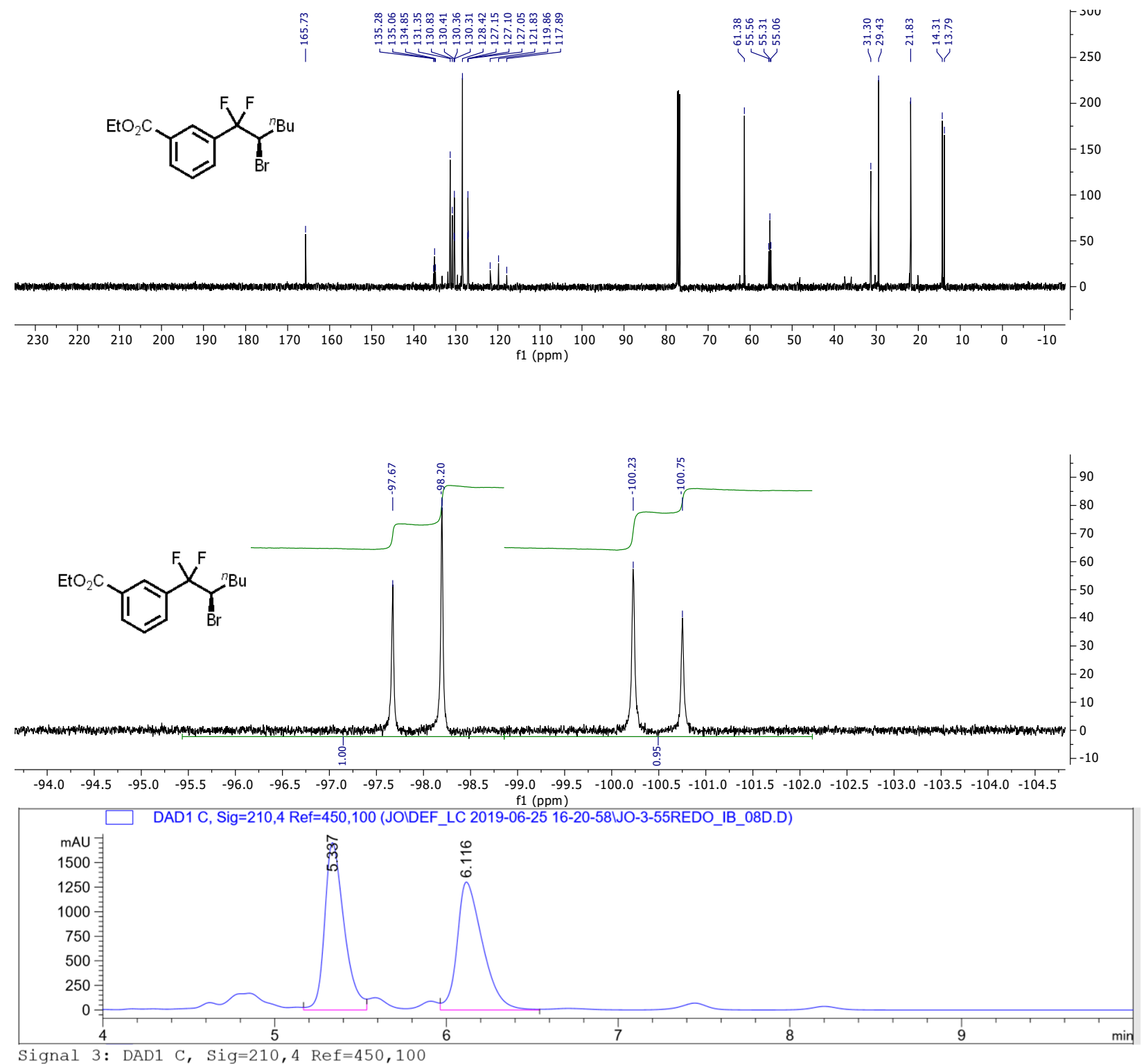

$\begin{array}{cccccc}\begin{array}{c}\text { Peak RetTime Type } \\ \text { \# } \\ {[\mathrm{min}]}\end{array} & \begin{array}{c}\text { Width } \\ {[\mathrm{min}]}\end{array} & \begin{array}{c}\text { Area } \\ {\left[\mathrm{mAU}{ }^{*} \mathrm{~S}\right]}\end{array} & \begin{array}{c}\text { Height } \\ {[\mathrm{mAU}]}\end{array} & \begin{array}{c}\text { Area } \\ \frac{\circ}{0}\end{array} \\ -1 & 5.337 \text { VV } & 0.1199 & 1.34405 \mathrm{e} 4 & 1705.81982 & 50.0102 \\ 2 & 6.116 \mathrm{VV} & 0.1562 & 1.34350 \mathrm{e} 4 & 1303.77161 & 49.9898\end{array}$<smiles>CC(Br)C(F)(F)c1cccc(C(F)(F)F)c1</smiles>

(R)-1-(2-bromo-1,1-difluoropropyl)-3-(trifluoromethyl)benzene. 1e was prepared from 2e $(53.0 \mathrm{mg}, 0.2 \mathrm{mmol})$ according to the General Procedure as a clear, colorless oil (36.4 $\mathrm{mg}, 60 \%$ yield).

${ }^{1} \mathrm{H}$ NMR $\left(500 \mathrm{MHz}, \mathrm{CDCl}_{3}\right) \delta 7.81-7.72(\mathrm{~m}, 3 \mathrm{H}), 7.64-7.59(\mathrm{~m}, 1 \mathrm{H}), 4.42-4.31(\mathrm{~m}, 1 \mathrm{H}), 1.76(\mathrm{~d}, J=6.9 \mathrm{~Hz}$, $3 \mathrm{H})$.

${ }^{13} \mathrm{C}$ NMR $\left(126 \mathrm{MHz}, \mathrm{CDCl}_{3}\right) \delta 135.13(\mathrm{t}, J=27.2 \mathrm{~Hz}), 130.95(\mathrm{q}, J=33.1 \mathrm{~Hz}), 129.56(\mathrm{t}, J=6.1 \mathrm{~Hz}), 128.94$, $127.24(\mathrm{~d}, J=4.1 \mathrm{~Hz}), 124.70(\mathrm{q}, J=272.7,271.2 \mathrm{~Hz}), 123.14(\mathrm{tt}, J=7.2,3.6 \mathrm{~Hz}), 119.80(\mathrm{dd}, J=249.0,248.4$ $\mathrm{Hz}), 47.68(\mathrm{t}, J=32.9 \mathrm{~Hz}), 19.34$.

${ }^{19} \mathrm{~F}$ NMR $\left(471 \mathrm{MHz}, \mathrm{CDCl}_{3}\right) \delta-62.81(\mathrm{~s}, 3 \mathrm{~F}),-99.59(\mathrm{~d}, J=247.5 \mathrm{~Hz}, 1 \mathrm{~F}),-103.00(\mathrm{~d}, J=247.9 \mathrm{~Hz}, 1 \mathrm{~F})$. 
${ }^{19} \mathrm{~F}$ NMR (471 MHz, Chloroform- $d$ ) $\delta-62.81,-99.59$ (d, $\left.J=247.5 \mathrm{~Hz}\right),-103.00(\mathrm{~d}, J=247.9 \mathrm{~Hz})$.

HRMS (EI): for $\mathrm{C}_{10} \mathrm{H}_{8} \mathrm{BrF}_{5}$, [M] calculated $\mathrm{m} / \mathrm{z}=301.9724$ and 303.9704 , found $\mathrm{m} / \mathrm{z}=301.9722$ and 303.9700 Chiral GC: CP-Chirasil-Dex CB, $40{ }^{\circ} \mathrm{C}$ to $110{ }^{\circ} \mathrm{C}, 1{ }^{\circ} \mathrm{C} / \mathrm{min}, 7 \mathrm{psi}, 80 \%$ ee
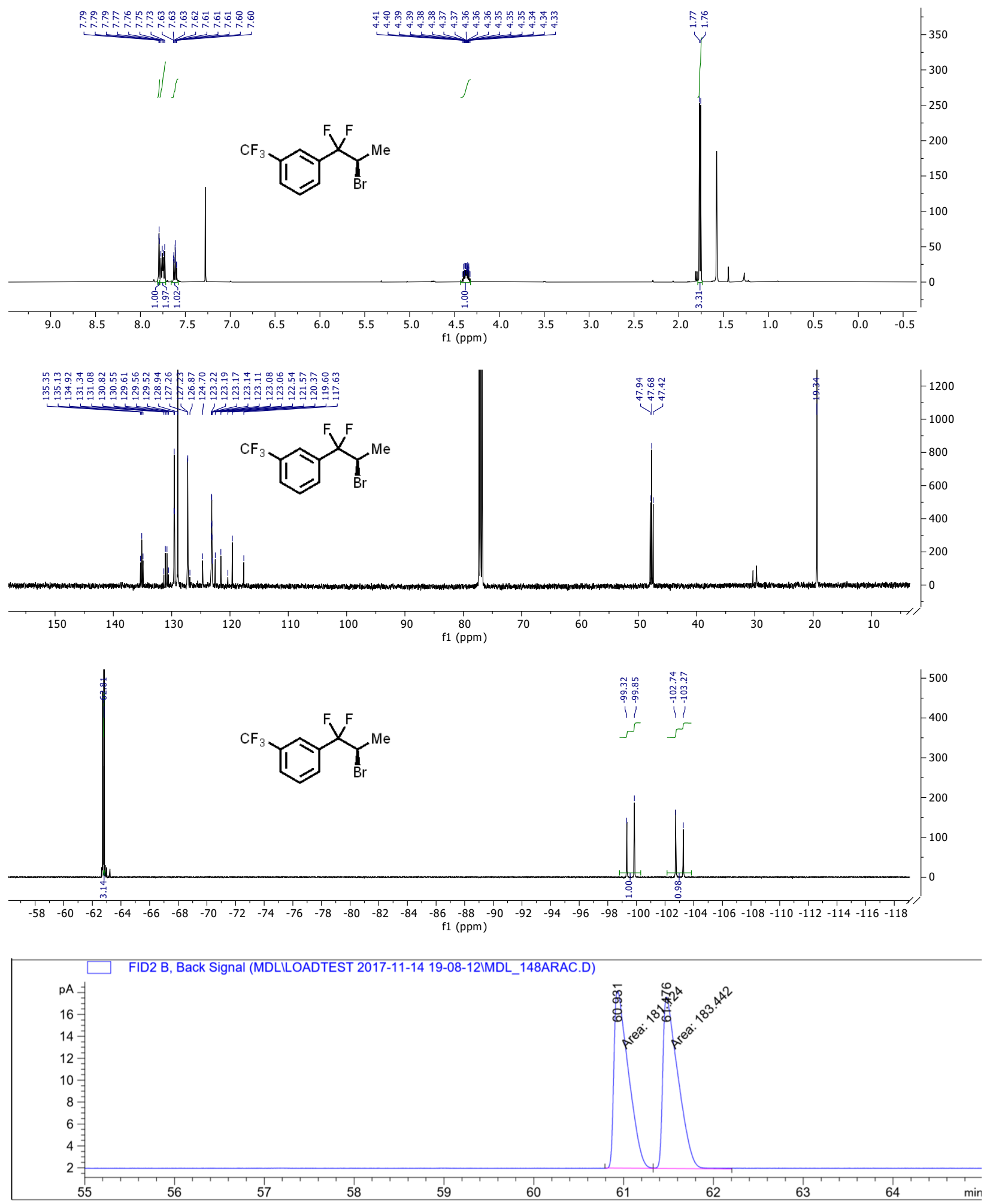

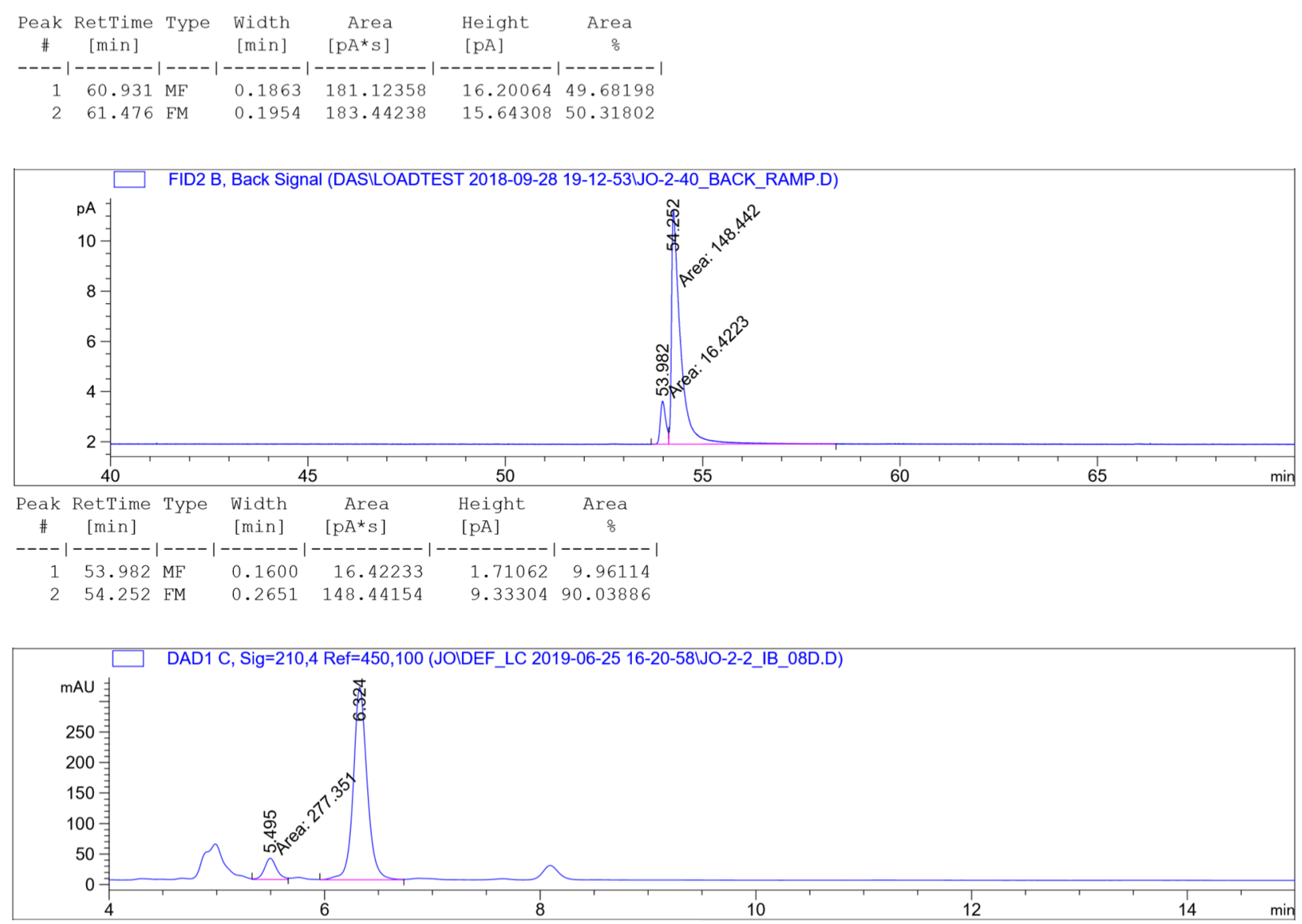

Signal 3: DAD1 C, Sig=210,4 Ref=450,100

\begin{tabular}{|c|c|c|c|c|c|c|}
\hline $\begin{array}{c}\text { Peak } \\
\#\end{array}$ & $\begin{array}{c}\text { RetTime } \\
\text { [min] }\end{array}$ & Type & $\begin{array}{l}\text { Width } \\
\text { [min] }\end{array}$ & $\begin{array}{c}\text { Area } \\
{\left[\mathrm{mAU}{ }^{*} \mathrm{~s}\right]}\end{array}$ & $\begin{array}{l}\text { Height } \\
{[\mathrm{mAU}]}\end{array}$ & $\begin{array}{c}\text { Area } \\
\quad \frac{\circ}{0}\end{array}$ \\
\hline$-1-$ & ---- & & ------ & --------- & -------- & -------- \\
\hline 1 & 5.495 & MM & 0.1316 & 277.35074 & 35.13698 & 3.9031 \\
\hline 2 & 6.324 & VB & 0.1328 & 2837.85522 & 315.79031 & 91.0969 \\
\hline
\end{tabular}<smiles>CCC(C)C(Br)C(F)(F)c1cccc(C#N)c1</smiles>

(R)-3-(2-bromo-1,1-difluorohexyl)benzonitrile. 1f was prepared from $\mathbf{2 f}(52.8 \mathrm{mg}, 0.2 \mathrm{mmol})$ according the General Procedure as a clear, colorless oil (39.3 $\mathrm{mg}, 65 \%$ yield).

${ }^{1} \mathrm{H}$ NMR $\left(500 \mathrm{MHz}, \mathrm{CDCl}_{3}\right) \delta 7.82(\mathrm{~s}, 1 \mathrm{H}), 7.80-7.73(\mathrm{~m}, 2 \mathrm{H}), 7.61-7.56(\mathrm{~m}, 1 \mathrm{H}), 4.17(\mathrm{qd}, J=11.1,2.7 \mathrm{~Hz}$, $1 \mathrm{H}), 2.08-1.97(\mathrm{~m}, 1 \mathrm{H}), 1.78-1.58(\mathrm{~m}, 2 \mathrm{H}), 1.47-1.27(\mathrm{~m}, 3 \mathrm{H}), 0.92(\mathrm{t}, J=7.2 \mathrm{~Hz}, 3 \mathrm{H})$.

${ }^{13} \mathrm{C}$ NMR $\left(126 \mathrm{MHz}, \mathrm{CDCl}_{3}\right) \delta 136.15(\mathrm{t}, J=27.5 \mathrm{~Hz}), 133.83,130.52(\mathrm{t}, J=6.1 \mathrm{~Hz}), 130.00(\mathrm{t}, J=6.4 \mathrm{~Hz}), 129.23$, $119.36(\mathrm{t}, J=248.9 \mathrm{~Hz}), 117.94,112.78,54.64(\mathrm{t}, J=31.5 \mathrm{~Hz}), 31.18,29.38,21.83,13.79$.

${ }^{19} \mathrm{~F}$ NMR $\left(471 \mathrm{MHz}, \mathrm{CDCl}_{3}\right) \delta-96.08(\mathrm{~d}, J=248.2 \mathrm{~Hz}, 1 \mathrm{~F}),-103.11(\mathrm{~d}, J=248.6 \mathrm{~Hz}, 1 \mathrm{~F})$.

HRMS (ESI): for $\mathrm{C}_{13} \mathrm{H}_{15} \mathrm{BrF}_{2} \mathrm{~N},[\mathrm{M}+\mathrm{H}]^{+}$calculated $\mathrm{m} / z=302.0350$ and 304.0330 , found $\mathrm{m} / \mathrm{z}=302.0348$ and 304.0327

Chiral HPLC: Chiralpak AS-H, 1.0\% isopropanol/hexanes, $1.0 \mathrm{ml} / \mathrm{min}, 81 \%$ ee 

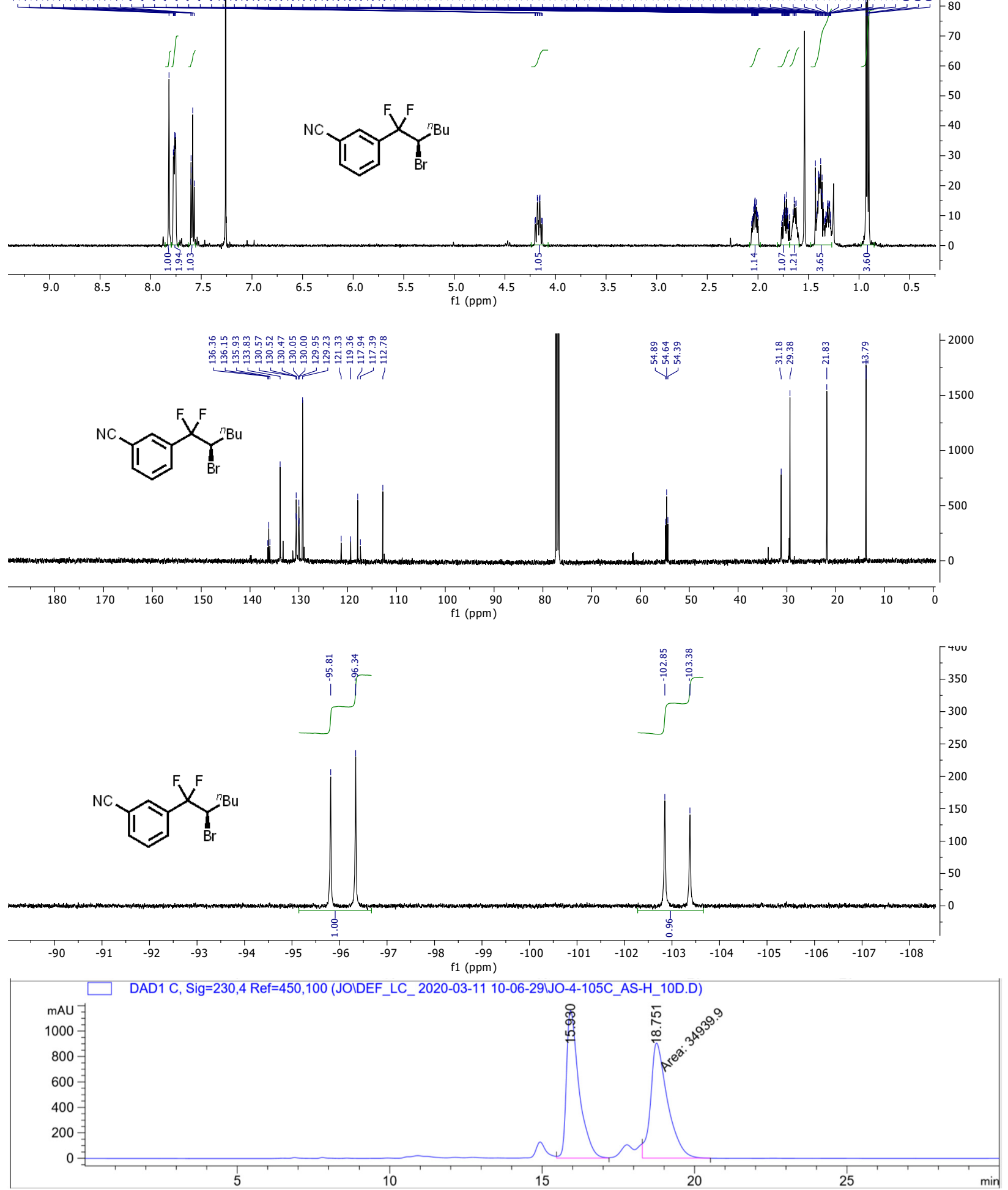

Signal 3: DAD1 C, Sig=230,4 Ref $=450,100$

\begin{tabular}{|c|c|c|c|c|c|c|}
\hline $\begin{array}{c}\text { Peak } \\
\#\end{array}$ & $\begin{array}{l}\text { RetTime } \\
\text { [min] }\end{array}$ & Type & $\begin{array}{l}\text { Width } \\
\text { [min] }\end{array}$ & $\begin{array}{c}\text { Area } \\
{\left[\mathrm{mAU}{ }^{\star} \mathrm{S}\right]}\end{array}$ & $\begin{array}{l}\text { Height } \\
\text { [mAU] }\end{array}$ & $\begin{array}{c}\text { Area } \\
\frac{\circ}{0}\end{array}$ \\
\hline & & & & (1) & 1 & ----1 \\
\hline 1 & 15.930 & VB & 0.4262 & $3.33953 \mathrm{e} 4$ & 1159.01672 & 8698 \\
\hline 2 & 18.751 & FM & 0.6433 & $3.49399 e 4$ & 905.23975 & 51.1302 \\
\hline
\end{tabular}




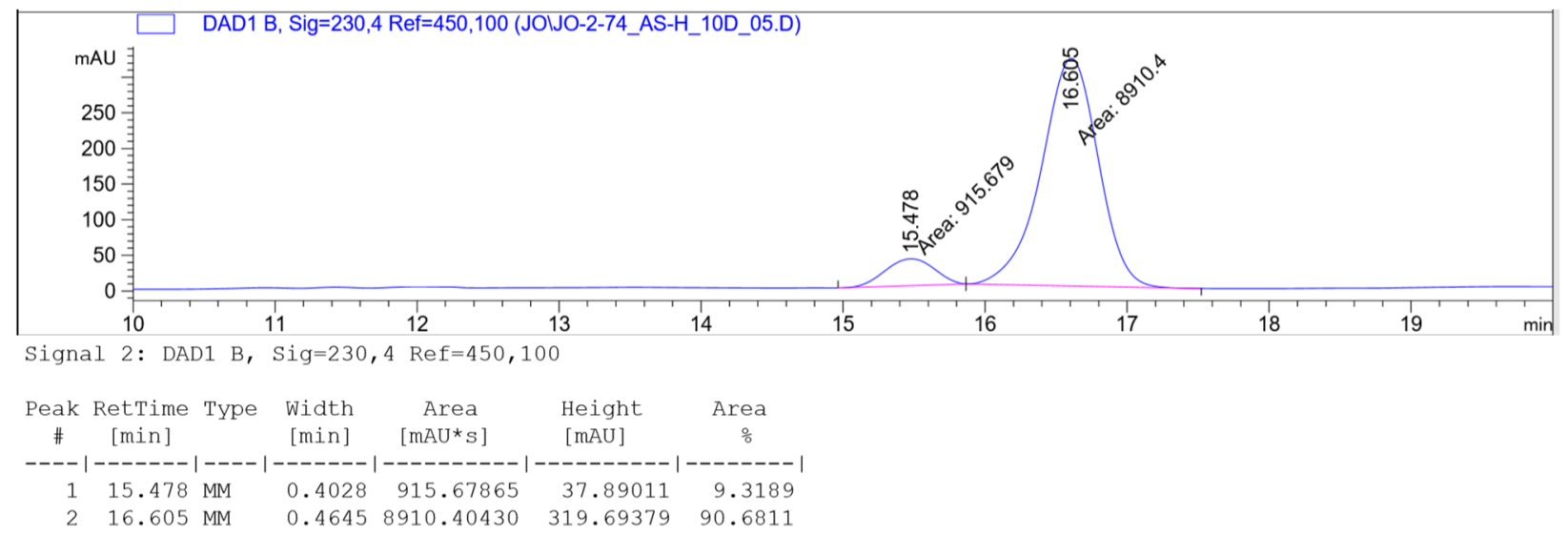

(R)-1-bromo-3-(2-bromo-1,1-difluorobutyl)benzene. $1 \mathrm{~g}$ was prepared from $2 \mathrm{~g}(58.0 \mathrm{mg}, 0.2 \mathrm{mmol})$ according to the General Procedure as a clear, colorless oil $(34.1 \mathrm{mg}, 52 \%$ yield).

${ }^{1} \mathrm{H}$ NMR $\left(500 \mathrm{MHz}, \mathrm{CDCl}_{3}\right) \delta 7.66(\mathrm{t}, J=1.9 \mathrm{~Hz}, 1 \mathrm{H}), 7.60(\mathrm{dd}, J=7.8,2.0 \mathrm{~Hz}, 1 \mathrm{H}), 7.45(\mathrm{~d}, J=7.6 \mathrm{~Hz}, 1 \mathrm{H}), 7.32$ $(\mathrm{t}, J=7.9 \mathrm{~Hz}, 1 \mathrm{H}), 4.11(\mathrm{qd}, J=11.2,2.7 \mathrm{~Hz}, 1 \mathrm{H}), 2.18-1.95(\mathrm{~m}, 1 \mathrm{H}), 1.83-1.66(\mathrm{~m}, 1 \mathrm{H}), 1.09(\mathrm{td}, J=7.4,1.7$ $\mathrm{Hz}, 3 \mathrm{H})$.

${ }^{13} \mathrm{C}$ NMR $\left(126 \mathrm{MHz}, \mathrm{CDCl}_{3}\right) \delta 136.59(\mathrm{t}, J=27.0 \mathrm{~Hz}), 133.47,129.87,129.21,124.78(\mathrm{t}, J=6.2 \mathrm{~Hz}), 122.40$, $119.36(\mathrm{t}, J=247.9 \mathrm{~Hz}), 57.14(\mathrm{t}, J=31.1 \mathrm{~Hz}), 25.24(\mathrm{t}, J=2.5 \mathrm{~Hz}), 12.23$.

${ }^{19} \mathrm{~F}$ NMR $\left(471 \mathrm{MHz}, \mathrm{CDCl}_{3}\right) \delta-98.29(\mathrm{~d}, J=246.3 \mathrm{~Hz}),-99.83(\mathrm{~d}, J=246.3 \mathrm{~Hz})$.

HRMS (EI): for $\mathrm{C}_{10} \mathrm{H}_{10} \mathrm{Br}_{2} \mathrm{~F}_{2}$, [M] $]^{+}$calculated $\mathrm{m} / z=325.9112$ and 327.9091 and 329.9071 , found $\mathrm{m} / z=325.9109$ and 327.9087 and 329.9066

Chiral GC: CP-Chirasil-Dex CB, isothermal $80{ }^{\circ} \mathrm{C}, 14 \mathrm{psi}$, $90 \%$ ee
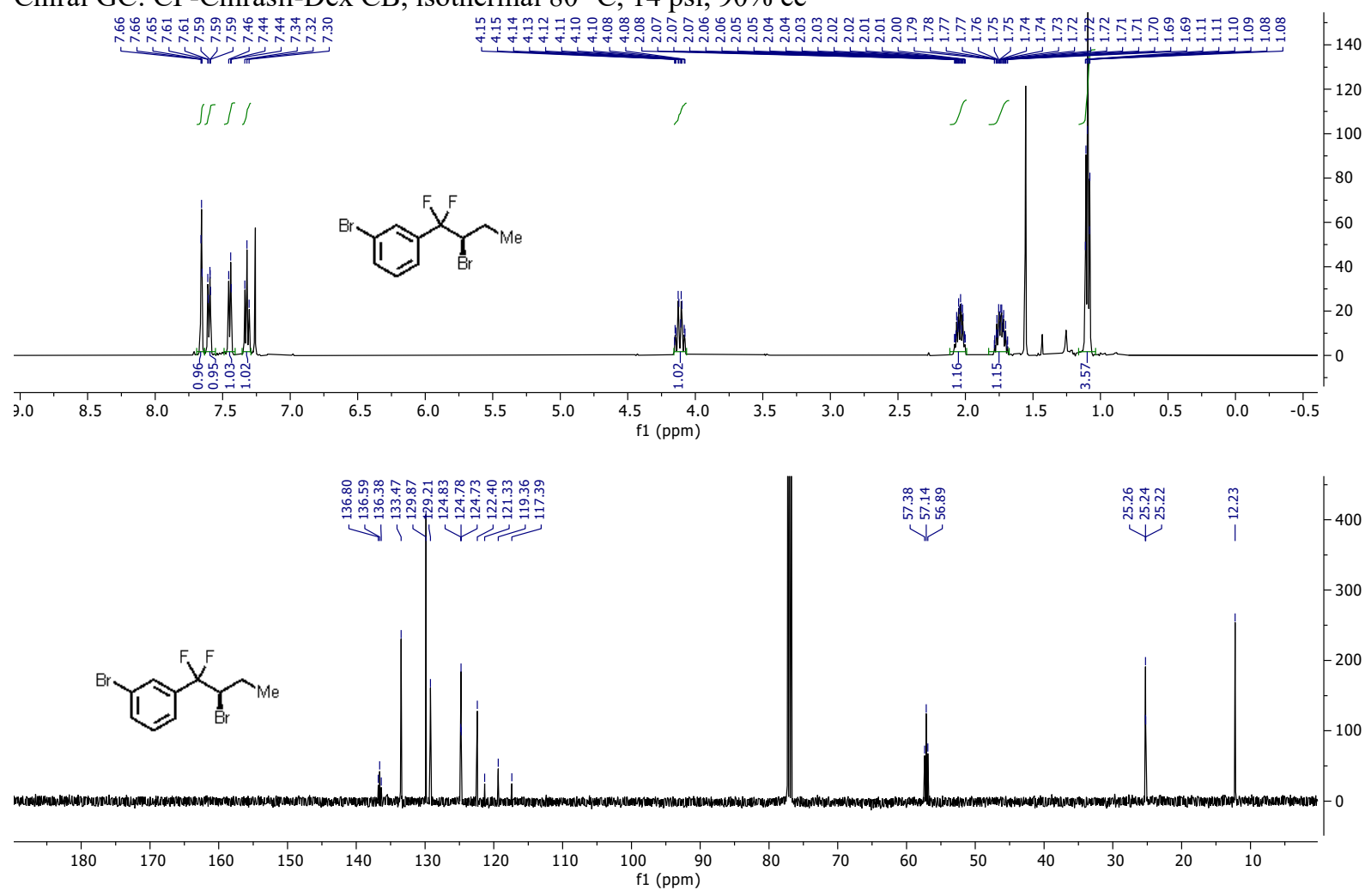

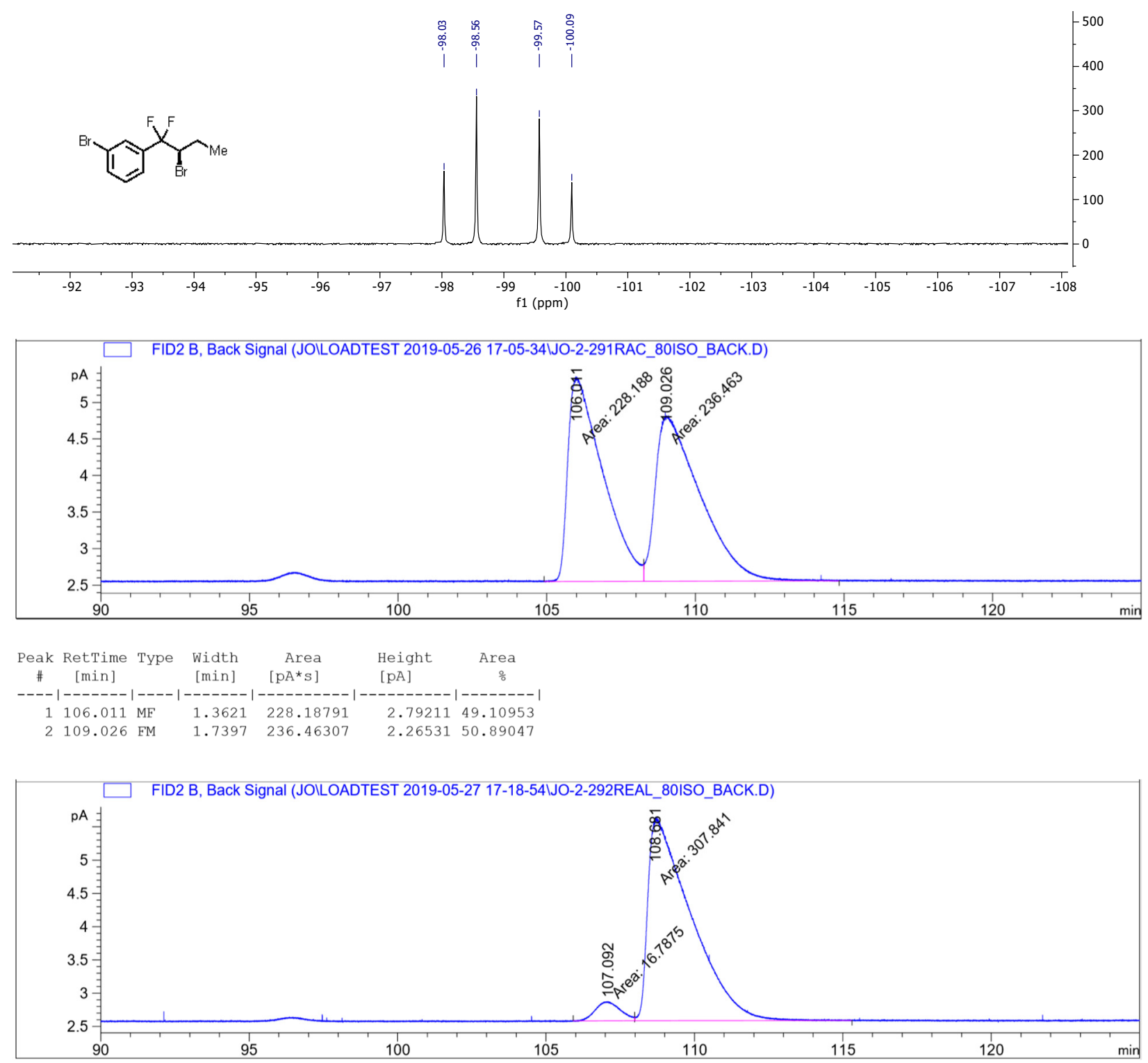

\begin{tabular}{|c|c|c|c|c|c|c|}
\hline $\begin{array}{c}\text { Peak } \\
\#\end{array}$ & $\begin{array}{c}\text { RetTime } \\
\text { [min] }\end{array}$ & Type & $\begin{array}{l}\text { Width } \\
\text { [min] }\end{array}$ & $\begin{array}{r}\text { Area } \\
{\left[\mathrm{pA}^{*} \mathrm{~S}\right]}\end{array}$ & $\begin{array}{l}\text { Height } \\
{[\mathrm{pA}]}\end{array}$ & $\begin{array}{c}\text { Area } \\
\frac{\circ}{0}\end{array}$ \\
\hline 1 & 107.092 & $\mathrm{MF}$ & 0.9568 & 16.78749 & $2.92438 e-1$ & 5.17130 \\
\hline 2 & 108.681 & FM & 1.6785 & 307.84058 & 3.05676 & 94.82870 \\
\hline
\end{tabular}<smiles>FC(Br)C(Br)C(F)(F)c1cccc(Br)c1</smiles>

(R)-1-bromo-3-(2-bromo-1,1-difluoro-3-methylbutyl)benzene. 1h was prepared from $\mathbf{2 h}$ (60.8 $\mathrm{mg}, 0.2 \mathrm{mmol})$ according to General Procedure A as a clear, colorless oil ( $49.9 \mathrm{mg}, 73 \%$ yield).

${ }^{1} \mathrm{H}$ NMR $\left(500 \mathrm{MHz}, \mathrm{CDCl}_{3}\right) \delta 7.65(\mathrm{~s}, 1 \mathrm{H}), 7.60(\mathrm{~d}, J=8.0 \mathrm{~Hz}, 1 \mathrm{H}), 7.44(\mathrm{~d}, J=7.8 \mathrm{~Hz}, 1 \mathrm{H}), 7.32(\mathrm{t}, J=7.9 \mathrm{~Hz}$, $1 \mathrm{H}), 4.20$ (ddd, $J=15.3,13.3,2.3 \mathrm{~Hz}, 1 \mathrm{H}), 2.02$ (pd, $J=6.5,2.2 \mathrm{~Hz}, 1 \mathrm{H}), 1.03$ (dd, $J=6.6,3.5 \mathrm{~Hz}, 6 \mathrm{H}$ ).

${ }^{13} \mathrm{C}$ NMR $\left(126 \mathrm{MHz}, \mathrm{CDCl}_{3}\right) \delta 137.51(\mathrm{t}, J=27.0 \mathrm{~Hz}), 133.48,130.06,128.79(\mathrm{t}, J=6.5 \mathrm{~Hz}), 124.34(\mathrm{t}, J=6.0 \mathrm{~Hz})$, $122.59,119.64$ (t, $J=249.0 \mathrm{~Hz}), 63.21$ (dd, $J=29.9,27.6 \mathrm{~Hz}), 28.73,22.58,17.79$. 
${ }^{19} \mathrm{~F}$ NMR $\left(471 \mathrm{MHz}, \mathrm{CDCl}_{3}\right) \delta-95.43(\mathrm{~d}, J=245.7 \mathrm{~Hz}),-98.59(\mathrm{~d}, J=247.1 \mathrm{~Hz})$.

HRMS (EI): for $\mathrm{C}_{11} \mathrm{H}_{12} \mathrm{Br}_{2} \mathrm{~F}_{2},[\mathrm{M}]^{+}$calculated $\mathrm{m} / \mathrm{z}=339.9268$ and 341.9248 and 343.9227 , found $\mathrm{m} / \mathrm{z}=339.9261$ and 341.9240 and 343.9219

Chiral GC: CP-Chirasil-Dex CB, $80{ }^{\circ} \mathrm{C}$ to $105^{\circ} \mathrm{C}, 0.5^{\circ} \mathrm{C} / \mathrm{min}, 14 \mathrm{psi}, 70 \%$ ee
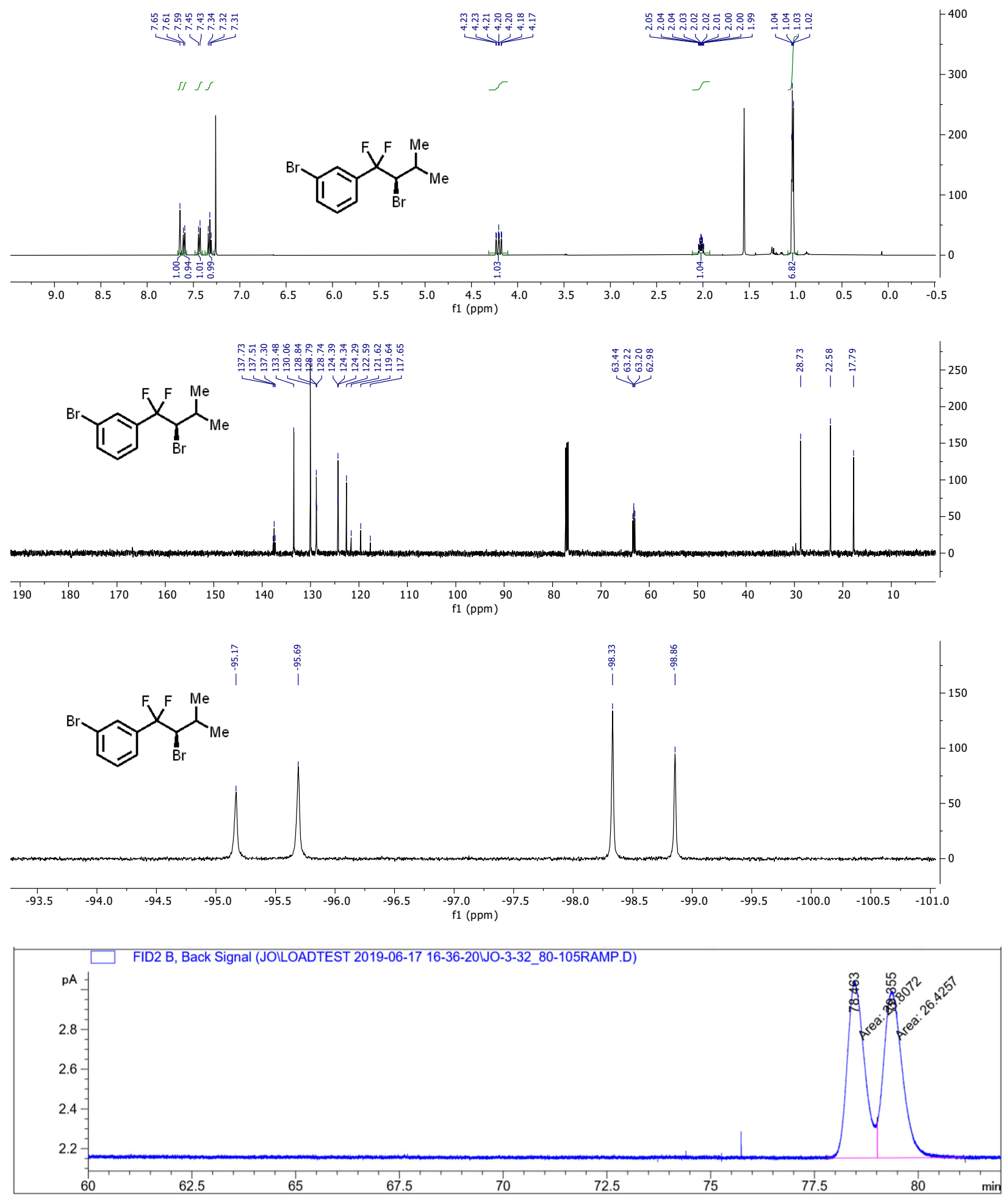


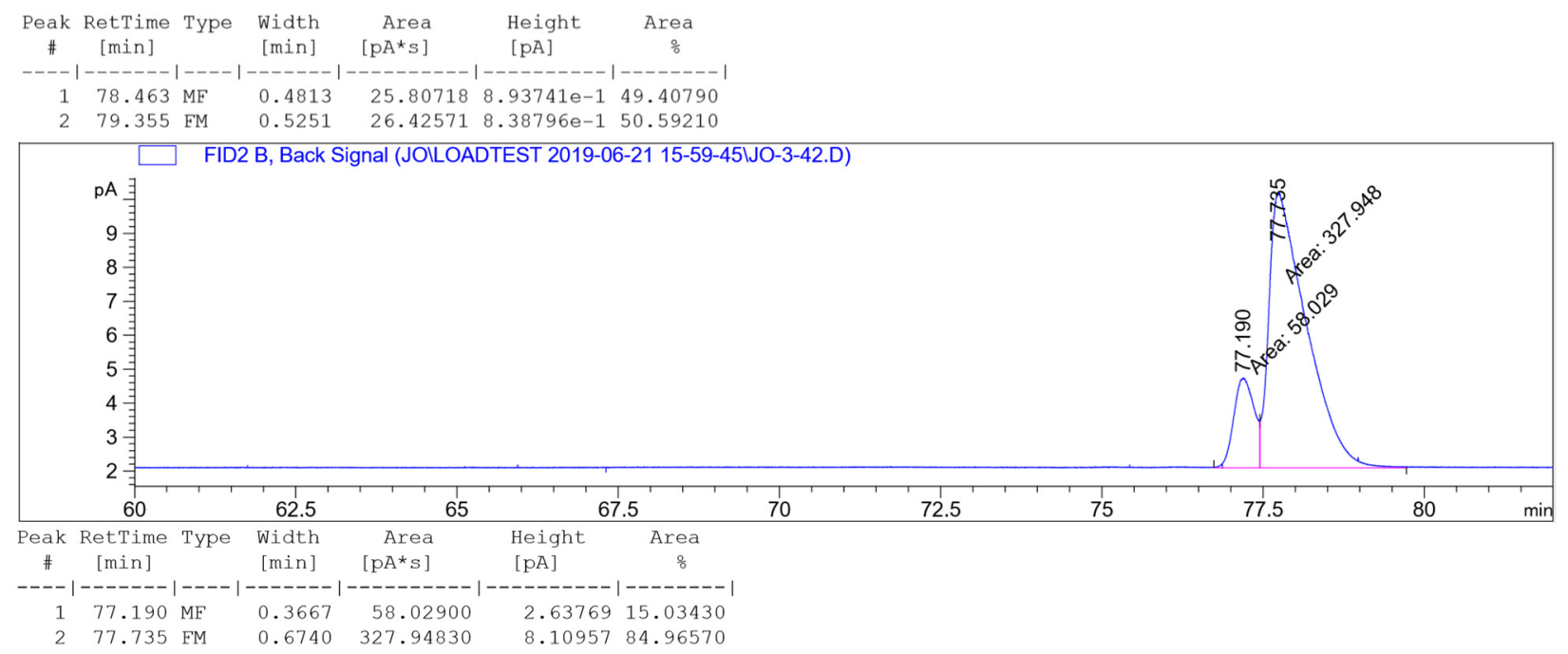

(R)-1-(2-bromo-1,1-difluorobutyl)-4-nitrobenzene. 1i was prepared from $2 \mathbf{i}(51.2 \mathrm{mg}, 0.2 \mathrm{mmol})$ according to the General Procedure as a white solid ( $48.8 \mathrm{mg}, 83 \%$ yield).

${ }^{1} \mathrm{H}$ NMR $\left(500 \mathrm{MHz}, \mathrm{CDCl}_{3}\right) \delta 8.31(\mathrm{~d}, J=8.7 \mathrm{~Hz}, 2 \mathrm{H}), 7.72(\mathrm{~d}, J=9.0 \mathrm{~Hz}, 2 \mathrm{H}), 4.14(\mathrm{dddd}, J=13.9,11.4,8.7,2.8$ $\mathrm{Hz}, 1 \mathrm{H}), 2.20-2.07(\mathrm{~m}, 1 \mathrm{H}), 1.76$ (dddd, $J=14.6,10.9,7.3,1.2 \mathrm{~Hz}, 1 \mathrm{H}), 1.12(\mathrm{t}, J=7.3 \mathrm{~Hz}, 3 \mathrm{H})$.

${ }^{13} \mathrm{C}$ NMR $\left(126 \mathrm{MHz}, \mathrm{CDCl}_{3}\right) \delta 149.08,140.72(\mathrm{t}, J=27.0 \mathrm{~Hz}), 127.52,123.44,119.49(\mathrm{t}, J=247.8 \mathrm{~Hz}), 56.46(\mathrm{t}, J$ $=30.9 \mathrm{~Hz}), 25.10,12.14$.

${ }^{19} \mathrm{~F}$ NMR (471 MHz, $\left.\mathrm{CDCl}_{3}\right) \delta-100.99$ (dd, $\left.J=248.4,6.3 \mathrm{~Hz}, 1 \mathrm{~F}\right),-107.86$ (dd, $\left.J=248.8,9.4 \mathrm{~Hz}, 1 \mathrm{~F}\right)$.

HRMS (EI): for $\mathrm{C}_{10} \mathrm{H}_{10} \mathrm{BrF}_{2} \mathrm{NO}_{2}$, [M] ${ }^{+}$calculated $\mathrm{m} / \mathrm{z}=292.9857$ and 294.9837 , found $\mathrm{m} / \mathrm{z}=292.9857$ and 294.9835

Chiral HPLC: Chiralcel OD-H, 1.0\% isopropanol/hexanes, $1.0 \mathrm{ml} / \mathrm{min}, 92 \%$ ee
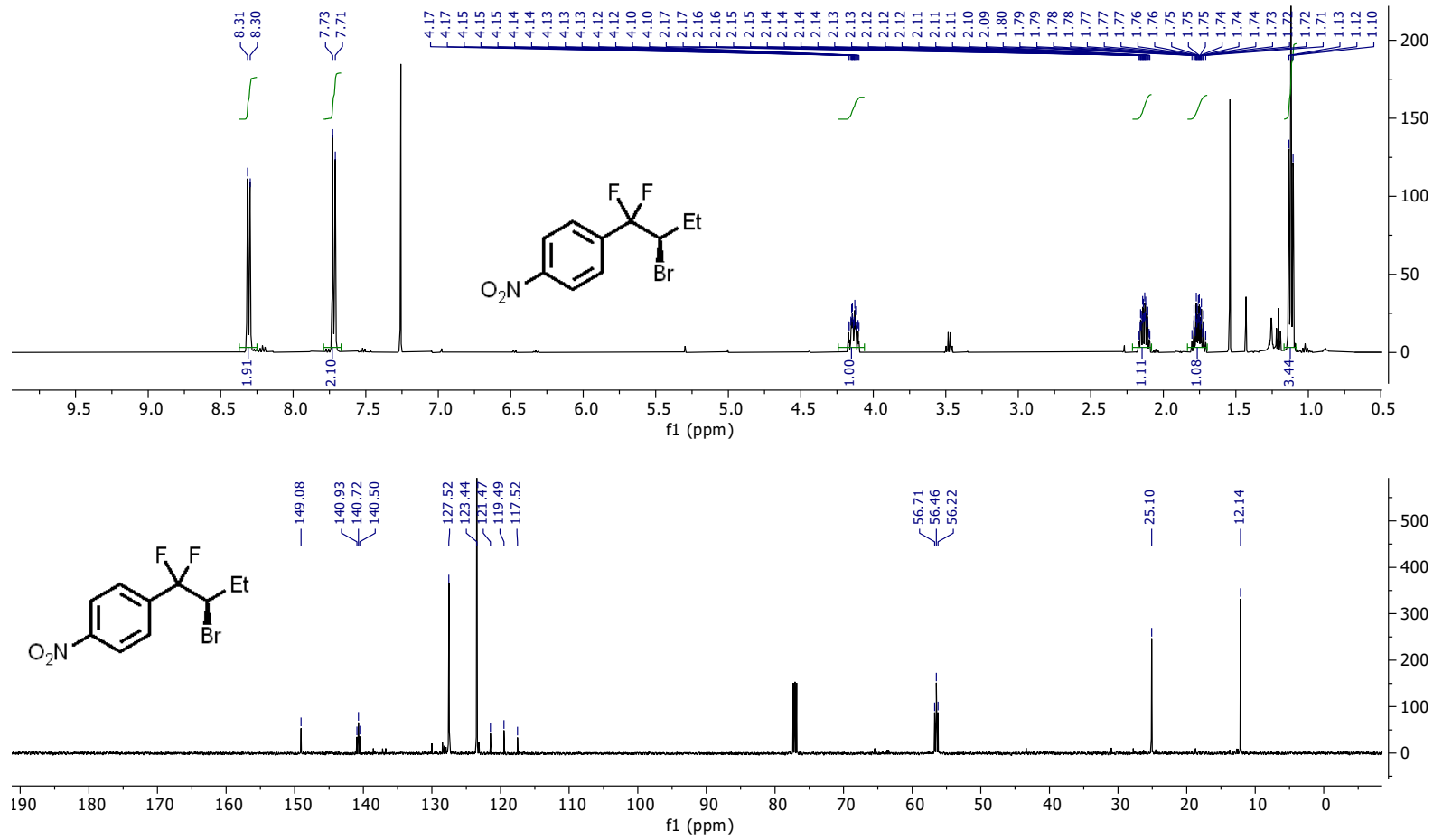

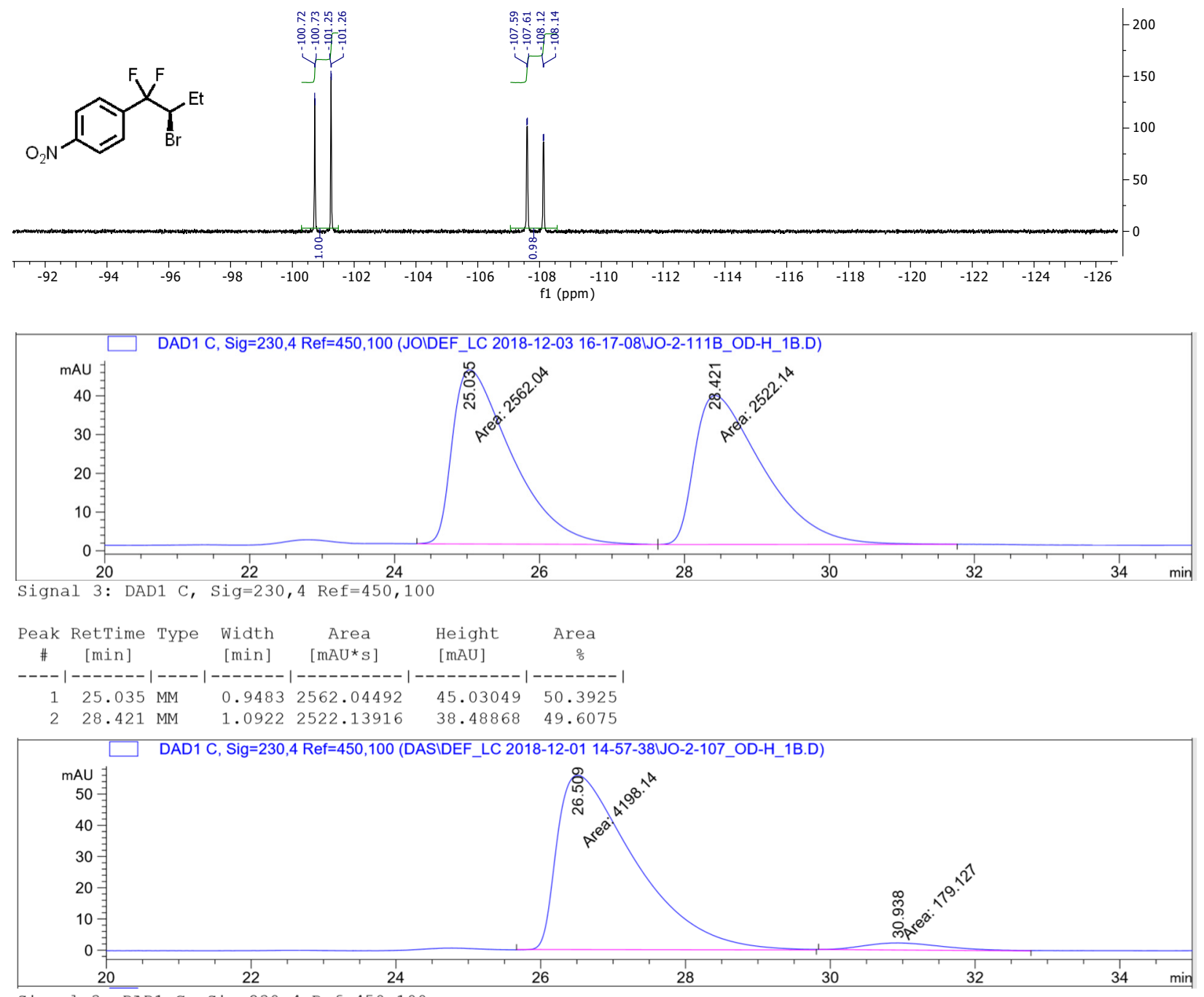

Signal 3: DAD1 C, Sig=230,4 Ref=450,100

\begin{tabular}{|c|c|c|c|c|c|c|}
\hline $\begin{array}{c}\text { Peak } \\
\quad \#\end{array}$ & $\begin{array}{l}\text { RetTime } \\
\text { [min] }\end{array}$ & Type & $\begin{array}{l}\text { Width } \\
\text { [min] }\end{array}$ & $\begin{array}{c}\text { Area } \\
{\left[\mathrm{mAU} U^{*} \mathrm{~s}\right]}\end{array}$ & $\begin{array}{l}\text { Height } \\
{[\mathrm{mAU}]}\end{array}$ & $\begin{array}{c}\text { Area } \\
\frac{\circ}{0}\end{array}$ \\
\hline- & ----- & & ------ & --------- & --------1 & ------- \\
\hline 1 & 26.509 & MM & 1.2520 & 4198.13623 & 55.88586 & 95.9078 \\
\hline 2 & 30.938 & MM & 1.2992 & 179.12723 & 2.29790 & 4.0922 \\
\hline
\end{tabular}<smiles>CC(C)(C)C(Br)C(F)(F)c1ccc([N+](=O)[O-])cc1</smiles>

(R)-1-(2-bromo-1,1-difluorohexyl)-4-nitrobenzene. $\mathbf{1} \mathbf{j}$ was prepared from $\mathbf{2} \mathbf{j}$ (56.8 $\mathrm{mg}, 0.2 \mathrm{mmol}$ ) according to the General Procedure as a clear, colorless oil ( $54.1 \mathrm{mg}, 84 \%$ yield).

${ }^{1} \mathrm{H}$ NMR $\left(500 \mathrm{MHz}, \mathrm{CDCl}_{3}\right) \delta 8.33(\mathrm{~d}, J=8.5 \mathrm{~Hz}, 2 \mathrm{H}), 7.74(\mathrm{~d}, J=8.8 \mathrm{~Hz}, 2 \mathrm{H}), 4.21$ (dddd, $J=14.0,11.3,8.6,2.8$ $\mathrm{Hz}, 1 \mathrm{H}), 2.11-1.97(\mathrm{~m}, 1 \mathrm{H}), 1.83-1.60(\mathrm{~m}, 2 \mathrm{H}), 1.48-1.24(\mathrm{~m}, 3 \mathrm{H}), 0.93(\mathrm{t}, J=7.2 \mathrm{~Hz}, 3 \mathrm{H})$.

${ }^{13} \mathrm{C}$ NMR $\left(126 \mathrm{MHz}, \mathrm{CDCl}_{3}\right) \delta 139.07(\mathrm{t}, J=27.1 \mathrm{~Hz}), 132.07,127.09(\mathrm{t}, J=6.2 \mathrm{~Hz}), 119.50(\mathrm{t}, J=248.1 \mathrm{~Hz})$, $117.89,114.46,54.56(\mathrm{t}, J=31.3 \mathrm{~Hz}), 31.18,29.37,21.82,13.78$.

${ }^{19} \mathrm{~F}$ NMR $\left(471 \mathrm{MHz}, \mathrm{CDCl}_{3}\right) \delta-96.13(\mathrm{~d}, J=248.2 \mathrm{~Hz}, 1 \mathrm{~F}),-103.17(\mathrm{~d}, J=248.1 \mathrm{~Hz}, 1 \mathrm{~F})$.

HRMS (EI): for $\mathrm{C}_{12} \mathrm{H}_{14} \mathrm{BrF}_{2} \mathrm{NO}_{2}$, [M] ${ }^{+}$calculated $m / z=321.0170$ and 323.0150 , found $m / z=321.0168$ and 323.0147 Chiral HPLC: Chiralcel OB-H, $0.5 \%$ isopropanol/hexanes, $1.0 \mathrm{ml} / \mathrm{min}, 93 \%$ ee 

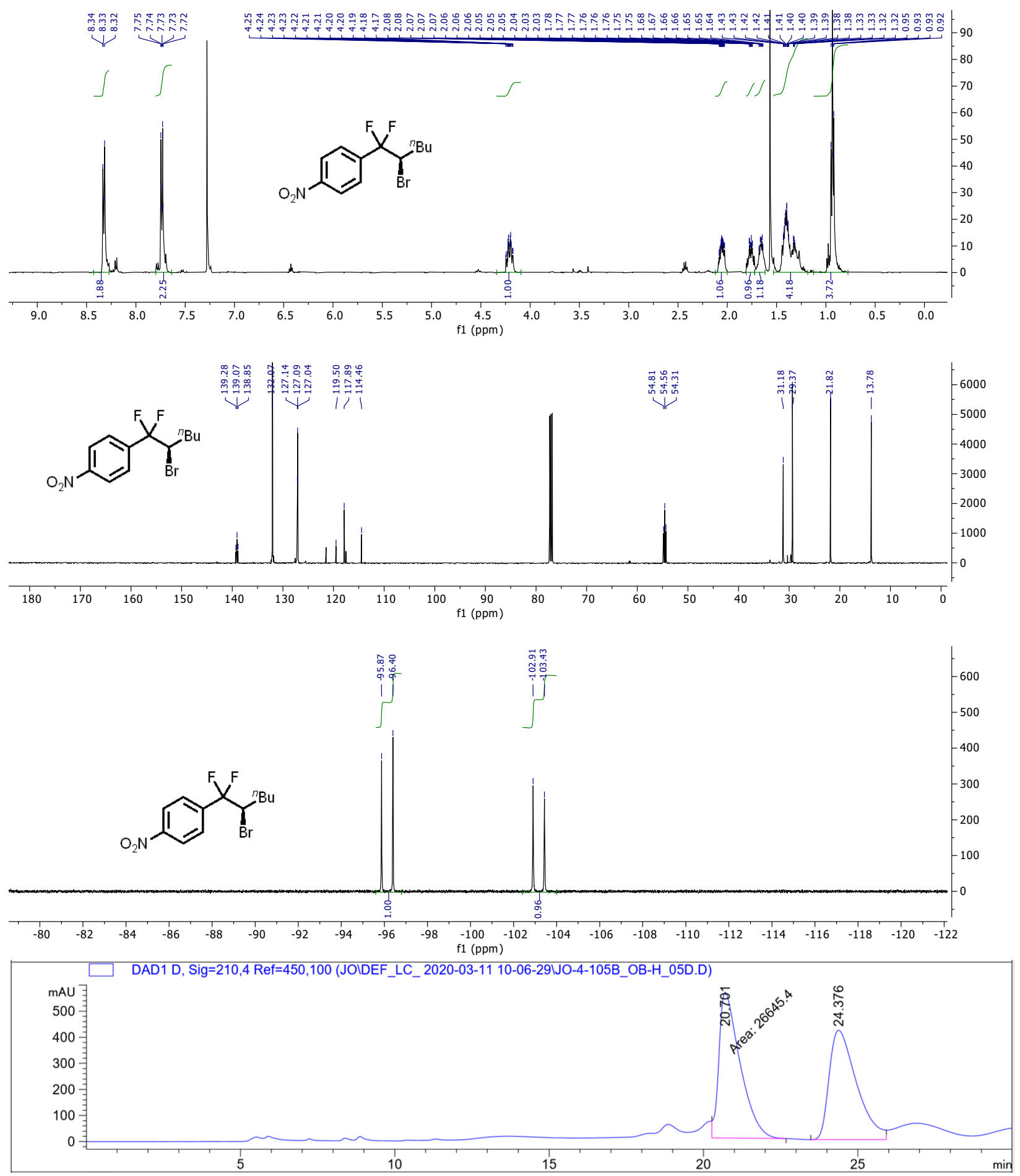

Signal 4: DAD1 D, Sig=210,4 Ref=450,100

\begin{tabular}{|c|c|c|c|c|c|c|}
\hline $\begin{array}{c}\text { Peak } \\
\#\end{array}$ & $\begin{array}{c}\text { RetTime } \\
\text { [min] }\end{array}$ & Type & $\begin{array}{c}\text { Width } \\
\text { [min] }\end{array}$ & $\begin{array}{c}\text { Area } \\
{\left[\mathrm{mAU}^{\star} \mathrm{S}\right]}\end{array}$ & $\begin{array}{l}\text { Height } \\
\text { [mAU] }\end{array}$ & $\begin{array}{c}\text { Area } \\
\frac{\circ}{0}\end{array}$ \\
\hline & ------- & & ------- & ------- & & -- \\
\hline 1 & 20.701 & FM & .7937 & 2.664 & 054 & 11 \\
\hline 2 & 24.376 & BV & .9300 & 2.58154 e 4 & 419.48834 & 49.2089 \\
\hline
\end{tabular}




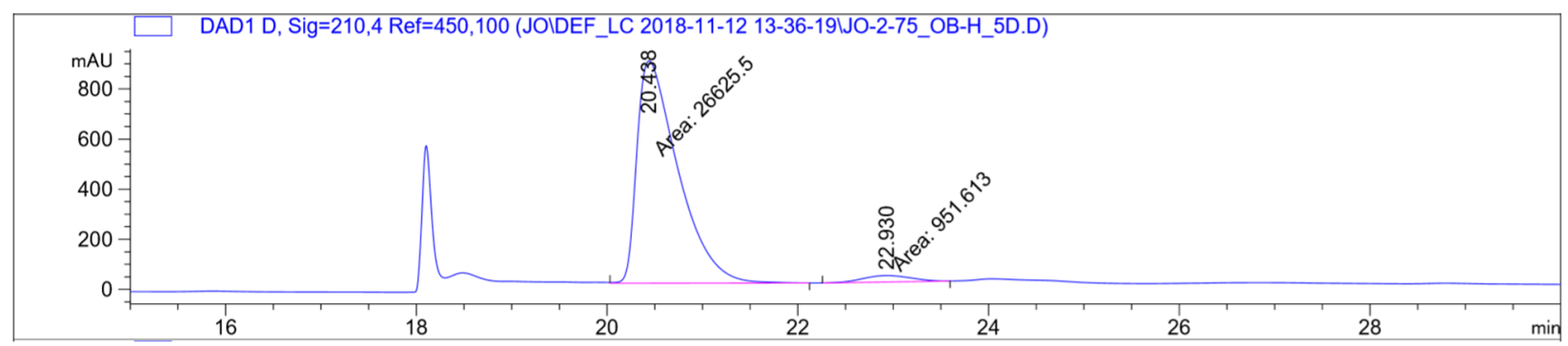

Signal 4: DAD1 D, Sig=210,4 Ref=450,100

\begin{tabular}{|c|c|c|c|c|c|c|}
\hline $\begin{array}{c}\text { Peak } \\
\#\end{array}$ & $\begin{array}{c}\text { RetTime } \\
\quad[\text { min] }\end{array}$ & Type & $\begin{array}{c}\text { Width } \\
\text { [min] }\end{array}$ & $\begin{array}{c}\text { Area } \\
{\left[\mathrm{mAU}^{\star} \mathrm{s}\right]}\end{array}$ & $\begin{array}{l}\text { Height } \\
\text { [mAU] }\end{array}$ & $\begin{array}{c}\text { Area } \\
\text { \% }\end{array}$ \\
\hline & ------ & & ------- & $\mid---------$ & ---------- & $--------\mid$ \\
\hline 1 & 20.438 & $\mathrm{MM}$ & 0.4996 & $2.66255 \mathrm{e} 4$ & 888.26093 & 96.5493 \\
\hline 2 & 22.930 & $\mathrm{MM}$ & 0.6040 & 951.61267 & 26.25781 & 3.4507 \\
\hline
\end{tabular}

(R)-4-(2-bromo-1,1-difluoropropyl)phenyl trifluoromethanesulfonate. 1k was prepared from $2 \mathbf{k}(69.0 \mathrm{mg}, 0.2 \mathrm{mmol})$ according to the General Procedure as a clear, colorless oil $(61.3 \mathrm{mg}, 80 \%$ yield).

${ }^{1} \mathrm{H}$ NMR $\left(500 \mathrm{MHz}, \mathrm{CDCl}_{3}\right) \delta 7.65(\mathrm{~d}, J=8.3 \mathrm{~Hz}, 2 \mathrm{H}), 7.38(\mathrm{~d}, J=8.5 \mathrm{~Hz}, 2 \mathrm{H}), 4.41-4.30(\mathrm{~m}, 1 \mathrm{H}), 1.75(\mathrm{~d}, J=$ $7.1 \mathrm{~Hz}, 3 \mathrm{H})$.

${ }^{13} \mathrm{C}$ NMR $\left(126 \mathrm{MHz}, \mathrm{CDCl}_{3}\right) \delta 150.66,134.49(\mathrm{t}, J=27.2 \mathrm{~Hz}), 128.48(\mathrm{t}, J=6.1 \mathrm{~Hz}), 121.34,119.54(\mathrm{t}, J=248.3$, $246.9 \mathrm{~Hz}), 118.32$ (q, $J=321.3,320.7,319.9 \mathrm{~Hz}), 47.63$ (t, $J=32.9 \mathrm{~Hz}), 19.34$.

${ }^{19} \mathrm{~F}$ NMR $\left(471 \mathrm{MHz}, \mathrm{CDCl}_{3}\right) \delta-72.78(\mathrm{~s}, 3 \mathrm{~F}),-98.92(\mathrm{~d}, J=247.1 \mathrm{~Hz}, 1 \mathrm{~F}),-102.80(\mathrm{~d}, J=247.1 \mathrm{~Hz}, 1 \mathrm{~F})$.

HRMS (EI): for $\mathrm{C}_{10} \mathrm{H}_{8} \mathrm{BrF}_{5} \mathrm{O}_{3} \mathrm{~S}$, [M] $]^{+}$calculated $\mathrm{m} / \mathrm{z}=381.9292$ and 383.9272 , found $\mathrm{m} / \mathrm{z}=381.9293$ and 383.9271 Chiral GC: CP-Chirasil-Dex CB, $40{ }^{\circ} \mathrm{C}$ to $200{ }^{\circ} \mathrm{C}, 1{ }^{\circ} \mathrm{C} / \mathrm{min}, 7 \mathrm{psi}, 80 \%$ ee
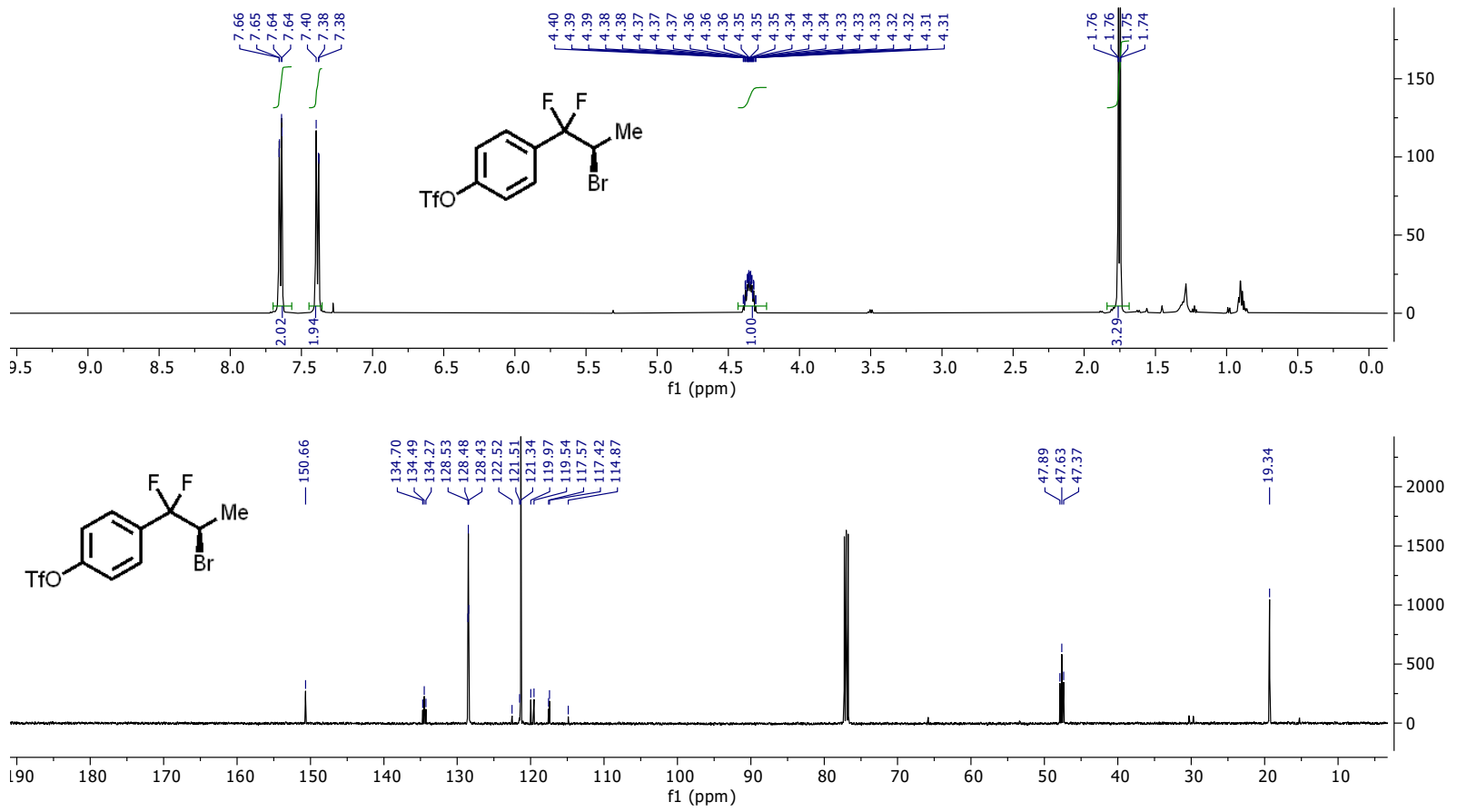

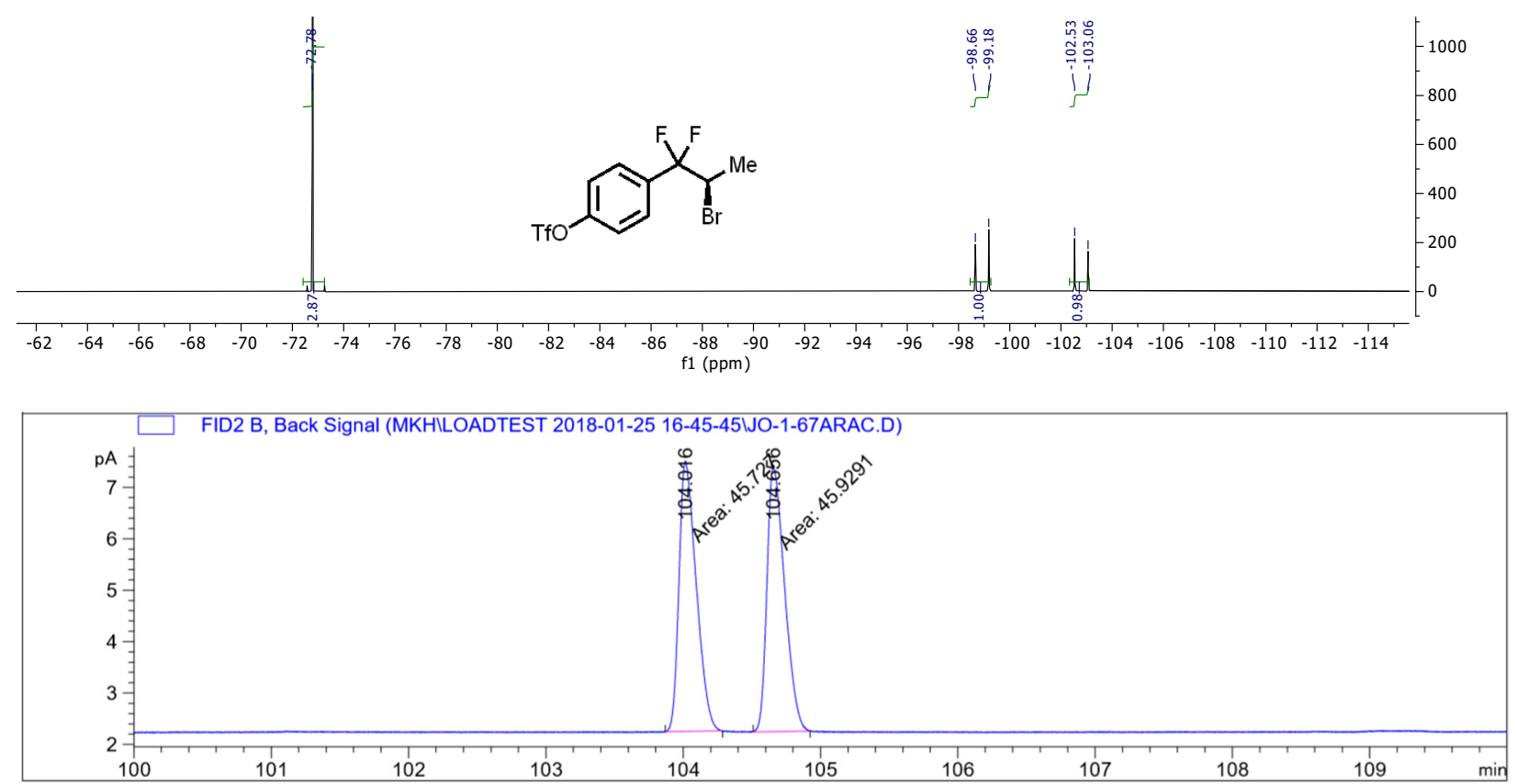

\begin{tabular}{|c|c|c|c|c|c|c|}
\hline $\begin{array}{c}\text { Peak } \\
\#\end{array}$ & $\begin{array}{c}\text { RetTime } \\
\text { [min] }\end{array}$ & Type & $\begin{array}{l}\text { Width } \\
\text { [min] }\end{array}$ & $\begin{array}{r}\text { Area } \\
{\left[\mathrm{pA} A^{*}\right]}\end{array}$ & $\begin{array}{l}\text { Height } \\
{[\mathrm{pA}]}\end{array}$ & $\begin{array}{c}\text { Area } \\
\frac{\circ}{0}\end{array}$ \\
\hline & & & & --------1 & --- & \\
\hline 1 & (1) & MM & 5 & 45.72702 & 5. & 974 \\
\hline 2 & 104.656 & MM & 0.1493 & 45.92914 & 5.12594 & 50.11026 \\
\hline
\end{tabular}

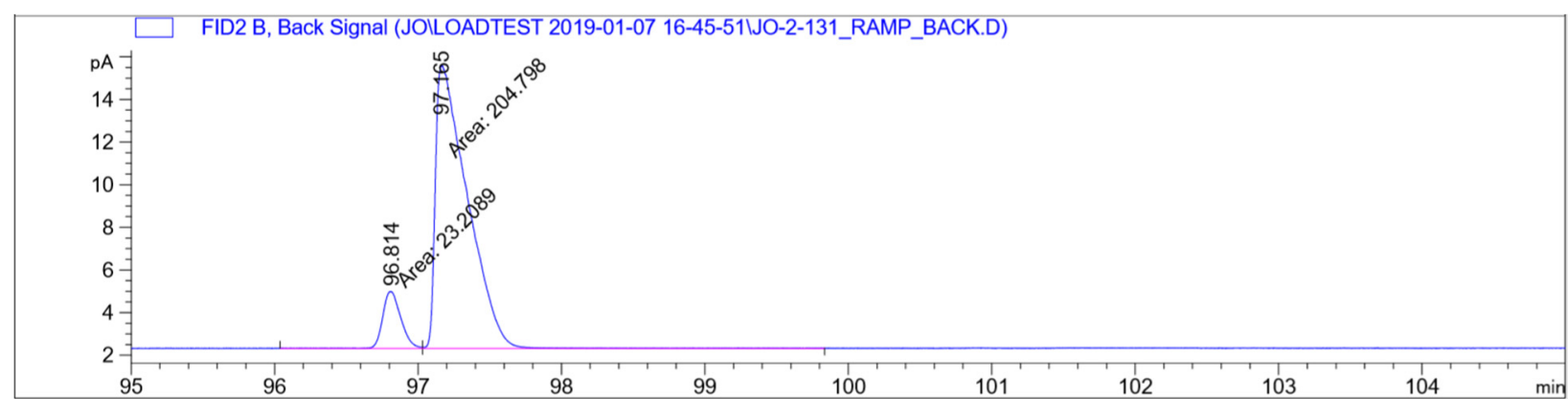

\begin{tabular}{|c|c|c|c|c|c|c|}
\hline $\begin{array}{c}\text { Peak } \\
\#\end{array}$ & $\begin{array}{c}\text { RetTime } \\
\text { [min] }\end{array}$ & Type & $\begin{array}{l}\text { Width } \\
\text { [min] }\end{array}$ & $\begin{array}{r}\text { Area } \\
{\left[\mathrm{pA}{ }^{*} \mathrm{~s}\right]}\end{array}$ & $\begin{array}{l}\text { Height } \\
{[\mathrm{pA}]}\end{array}$ & $\begin{array}{c}\text { Area } \\
\frac{\circ}{0}\end{array}$ \\
\hline 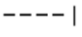 & & & & ---ー-ー-- & -------- & --1 \\
\hline 1 & 96.8 & $\mathrm{MF}$ & 0.1451 & 23.20891 & 2.66577 & 1903 \\
\hline 2 & 97.165 & FM & 0.2567 & 204.79826 & 13.29851 & 89.82097 \\
\hline
\end{tabular}<smiles>CCOC(=O)c1ccc(C(F)(F)C(Br)CC(C)(C)C)cc1</smiles>

(R)-ethyl 4-(2-bromo-1,1-difluorohexyl)benzoate. 11 was prepared from 21 (62.2 $\mathrm{mg}, 0.2 \mathrm{mmol})$ according to the General Procedure as a clear, colorless oil ( $58.7 \mathrm{mg}, 84 \%$ yield).

${ }^{1} \mathrm{H}$ NMR $\left(500 \mathrm{MHz}, \mathrm{CDCl}_{3}\right) \delta 8.12(\mathrm{~d}, J=8.1 \mathrm{~Hz}, 2 \mathrm{H}), 7.69-7.52(\mathrm{~m}, 2 \mathrm{H}), 4.40(\mathrm{q}, J=7.1 \mathrm{~Hz}, 2 \mathrm{H}), 4.20(\mathrm{qd}, J=$ $11.3,2.8 \mathrm{~Hz}, 1 \mathrm{H}), 1.96$ (dddd, $J=14.4,9.9,5.9,2.7 \mathrm{~Hz}, 1 \mathrm{H}), 1.73$ (dddd, $J=14.3,11.1,9.5,4.5 \mathrm{~Hz}, 1 \mathrm{H}), 1.62(\mathrm{qt}, J$ $=9.7,5.5 \mathrm{~Hz}, 1 \mathrm{H}), 1.41(\mathrm{t}, J=7.1 \mathrm{~Hz}, 3 \mathrm{H}), 1.39-1.22(\mathrm{~m}, 2 \mathrm{H}), 0.89(\mathrm{t}, J=7.2 \mathrm{~Hz}, 3 \mathrm{H})$. 
${ }^{13} \mathrm{C}$ NMR $\left(126 \mathrm{MHz}, \mathrm{CDCl}_{3}\right) \delta 165.75,138.69$ (t, $\left.J=26.5 \mathrm{~Hz}\right), 132.33,129.45,126.18(\mathrm{t}, J=6.1 \mathrm{~Hz}), 119.87$ (t, $J=$ 248.1, 247.3 Hz), 61.34, 55.14 (t, $J=31.2 \mathrm{~Hz}), 31.33,29.42,21.82,14.30,13.78$.

${ }^{19} \mathrm{~F}$ NMR $\left(471 \mathrm{MHz}, \mathrm{CDCl}_{3}\right) \delta-98.56(\mathrm{~d}, J=245.7 \mathrm{~Hz}, 1 \mathrm{~F}),-100.55(\mathrm{~d}, J=245.9 \mathrm{~Hz}, 1 \mathrm{~F})$.

HRMS (ESI): for $\mathrm{C}_{15} \mathrm{H}_{20} \mathrm{BrF}_{2} \mathrm{O}_{2},[\mathrm{M}+\mathrm{H}]^{+}$calculated $\mathrm{m} / \mathrm{z}=349.0609$ and 351.0589 , found $\mathrm{m} / z=349.0607$ and 351.0585.

Chiral HPLC: Chiralcel OJ-H, 0.3\% isopropanol/hexanes, $0.5 \mathrm{ml} / \mathrm{min}, 79 \%$ ee
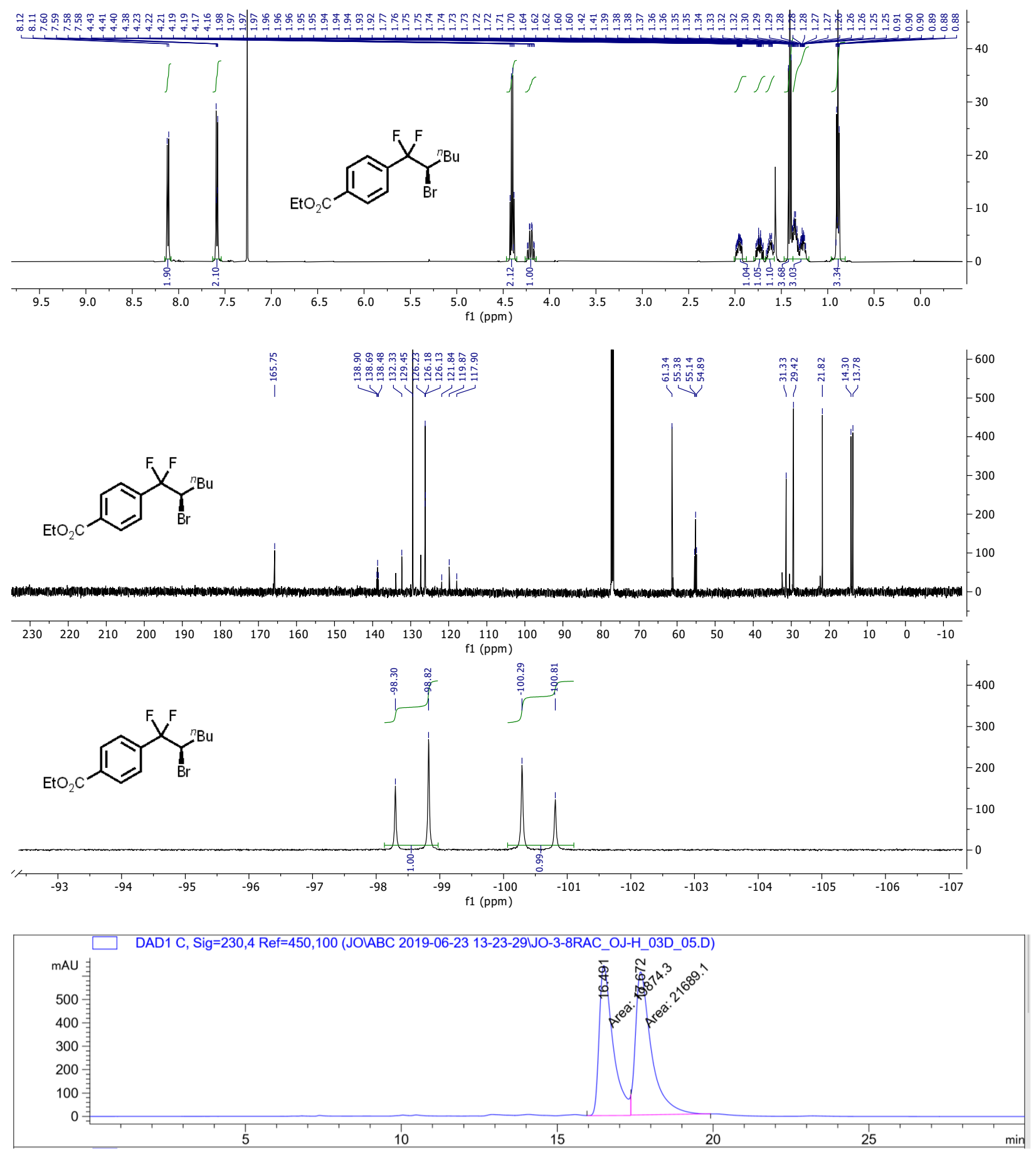

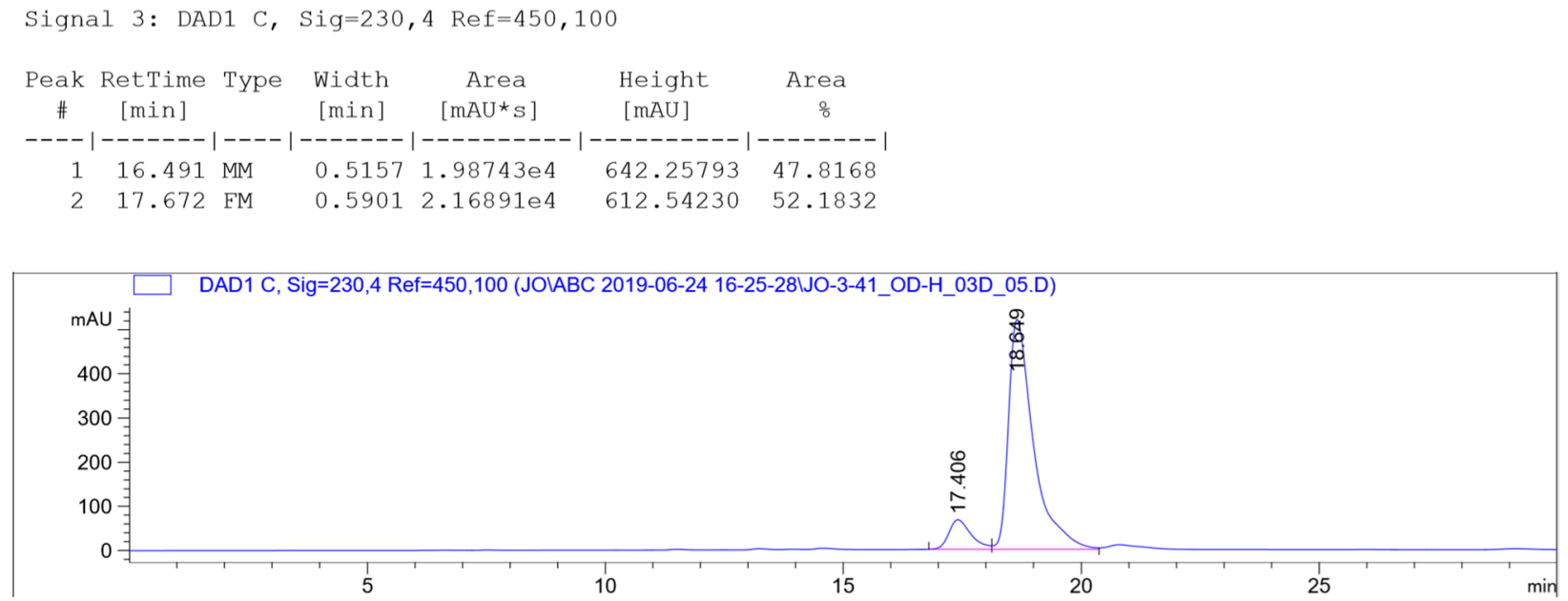

Signal 3: DAD1 C, Sig=230, 4 Ref=450,100

\begin{tabular}{|c|c|c|c|c|c|c|}
\hline $\begin{array}{c}\text { Peak } \\
\#\end{array}$ & $\begin{array}{c}\text { RetTime } \\
\text { [min] }\end{array}$ & Type & $\begin{array}{l}\text { Width } \\
\text { [min] }\end{array}$ & $\begin{array}{c}\text { Area } \\
{\left[\mathrm{mAU}^{\star} \mathrm{s}\right]}\end{array}$ & $\begin{array}{l}\text { Height } \\
\text { [mAU] }\end{array}$ & $\begin{array}{c}\text { Area } \\
\frac{\circ}{0}\end{array}$ \\
\hline 然 & & & & 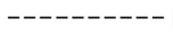 & -- & - \\
\hline 1 & & $3 V+2$ & & 29 & & \\
\hline 2 & 8.649 & VB & 0 & $1.90449 \mathrm{e} 4$ & 520.37158 & 6594 \\
\hline
\end{tabular}<smiles>CC(Br)C(F)(F)c1ccc(C(F)(F)F)cc1</smiles>

(R)-1-(2-bromo-1,1-difluoropropyl)-4-(trifluoromethyl)benzene. 1m was prepared from $\mathbf{2 m}$ (53.0 $\mathrm{mg}, 0.2 \mathrm{mmol})$ according to the General Procedure as a clear, colorless oil (33.3 $\mathrm{mg}, 55 \%$ yield).

${ }^{1} \mathrm{H}$ NMR $\left(500 \mathrm{MHz}, \mathrm{CDCl}_{3}\right) \delta 7.74(\mathrm{~d}, J=8.3 \mathrm{~Hz}, 2 \mathrm{H}), 7.67(\mathrm{~d}, J=8.2 \mathrm{~Hz}, 2 \mathrm{H}), 4.37$ (ddq, $J=12.2,10.1,6.9 \mathrm{~Hz}$, $1 \mathrm{H}), 1.76(\mathrm{~d}, J=6.9 \mathrm{~Hz}, 3 \mathrm{H})$.

${ }^{13} \mathrm{C}$ NMR (126 MHz, $\left.\mathrm{CDCl}_{3}\right) \delta 137.71(\mathrm{t}, J=26.8 \mathrm{~Hz}), 132.55(\mathrm{q}, J=32.4,31.7 \mathrm{~Hz}), 126.73(\mathrm{t}, J=6.1 \mathrm{~Hz}), 125.34$ $(\mathrm{q}, J=3.8 \mathrm{~Hz}), 123.61(\mathrm{q}, J=272.4 \mathrm{~Hz}), 119.81(\mathrm{t}, J=247.9,247.2 \mathrm{~Hz}), 47.64(\mathrm{t}, J=32.8 \mathrm{~Hz}), 19.35$.

${ }^{19} \mathrm{~F}$ NMR $\left(471 \mathrm{MHz}, \mathrm{CDCl}_{3}\right) \delta-62.96(\mathrm{~d}, J=4.9 \mathrm{~Hz}, 3 \mathrm{~F}),-99.90(\mathrm{~d}, J=246.5 \mathrm{~Hz}, 1 \mathrm{~F}),-103.12(\mathrm{~d}, J=246.4 \mathrm{~Hz}$, $1 \mathrm{~F})$.

HRMS (EI): for $\mathrm{C}_{10} \mathrm{H}_{8} \mathrm{BrF}_{5}$, [M] $]^{+}$calculated $\mathrm{m} / z=301.9724$ and 303.9704 , found $\mathrm{m} / \mathrm{z}=301.9721$ and 303.9700 Chiral GC: CP-Chirasil-Dex CB, $40{ }^{\circ} \mathrm{C}$ to $200{ }^{\circ} \mathrm{C}, 1{ }^{\circ} \mathrm{C} / \mathrm{min}, 7 \mathrm{psi}, 83 \%$ ee

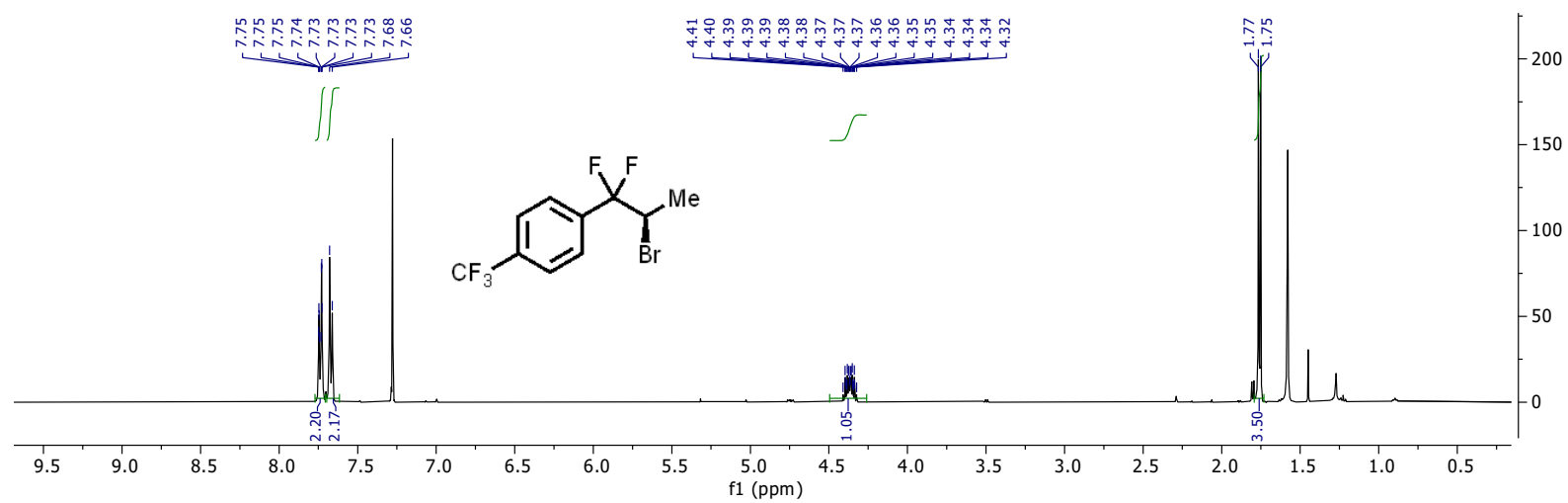



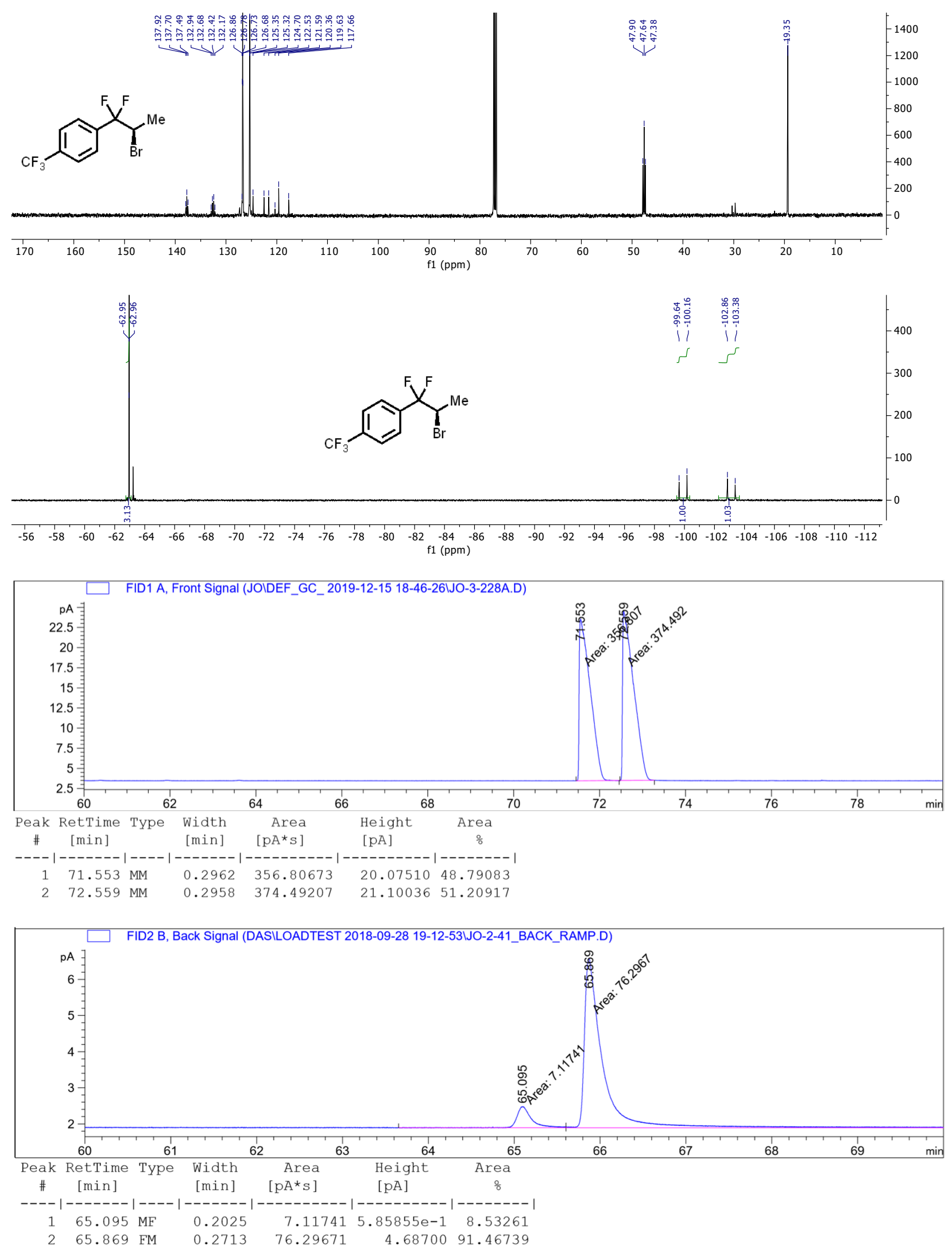
$\overbrace{B r}^{E t}$

(R)-4-(2-bromo-1,1-difluorobutyl)benzonitrile. 1n was prepared from $2 \mathbf{n}(47.2 \mathrm{mg}, 0.2 \mathrm{mmol})$ according to the General Procedure as a white solid $(40.0 \mathrm{mg}, 73 \%$ yield).

${ }^{1} \mathrm{H}$ NMR $\left(500 \mathrm{MHz}, \mathrm{CDCl}_{3}\right) \delta 7.82-7.72(\mathrm{~m}, 2 \mathrm{H}), 7.64(\mathrm{~d}, J=8.6 \mathrm{~Hz}, 2 \mathrm{H}), 4.11$ (dddd, $J=13.7,10.9,9.0,2.8 \mathrm{~Hz}$, $1 \mathrm{H}), 2.10(\mathrm{dqd}, J=14.5,7.3,2.9 \mathrm{~Hz}, 1 \mathrm{H}), 1.84-1.67(\mathrm{~m}, 1 \mathrm{H}), 1.11(\mathrm{t}, J=7.3 \mathrm{~Hz}, 3 \mathrm{H})$.

${ }^{13} \mathrm{C}$ NMR $\left(126 \mathrm{MHz}, \mathrm{CDCl}_{3}\right) \delta 139.07(\mathrm{t}, J=27.1 \mathrm{~Hz}), 132.08,127.07(\mathrm{t}, J=6.2 \mathrm{~Hz}), 119.46(\mathrm{dd}, J=249.2,248.3$ $\mathrm{Hz}), 117.88,114.48,56.50(\mathrm{t}, J=31.1 \mathrm{~Hz}), 25.11,12.17$.

${ }^{19} \mathrm{~F}$ NMR $\left(471 \mathrm{MHz}, \mathrm{CDCl}_{3}\right) \delta-97.02(\mathrm{~d}, J=248.2 \mathrm{~Hz}),-103.09(\mathrm{~d}, J=248.2 \mathrm{~Hz})$.

HRMS (EI): for $\mathrm{C}_{11} \mathrm{H}_{10} \mathrm{BrF}_{2} \mathrm{~N}$, [M] $]^{+}$calculated $\mathrm{m} / z=272.9959$ and 274.9939 , found $\mathrm{m} / z=272.9963$ and 274.9942 .

Chiral GC: CP-Chirasil-Dex CB, isothermal $140{ }^{\circ} \mathrm{C}, 7 \mathrm{psi}, 89 \%$ ee
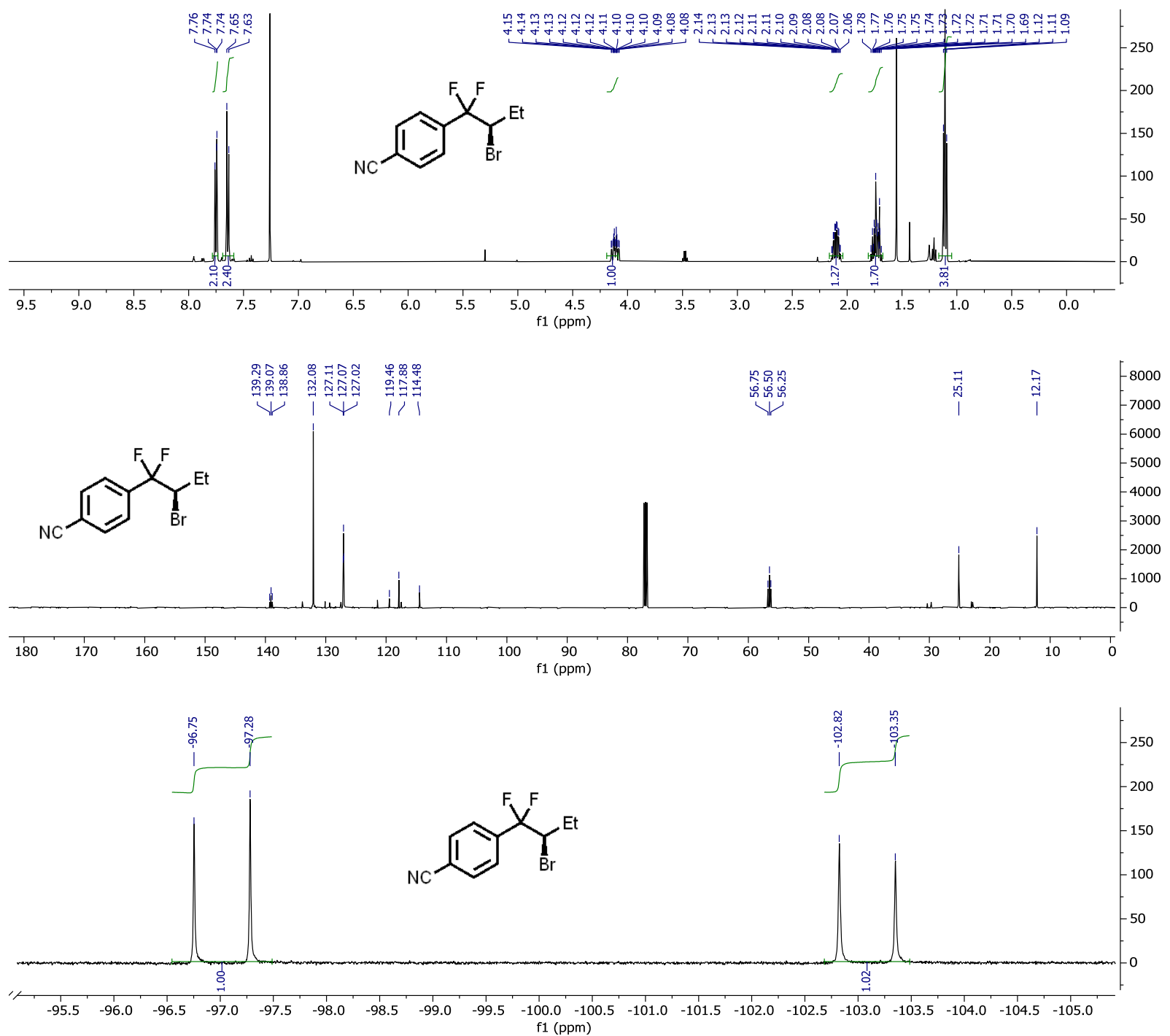

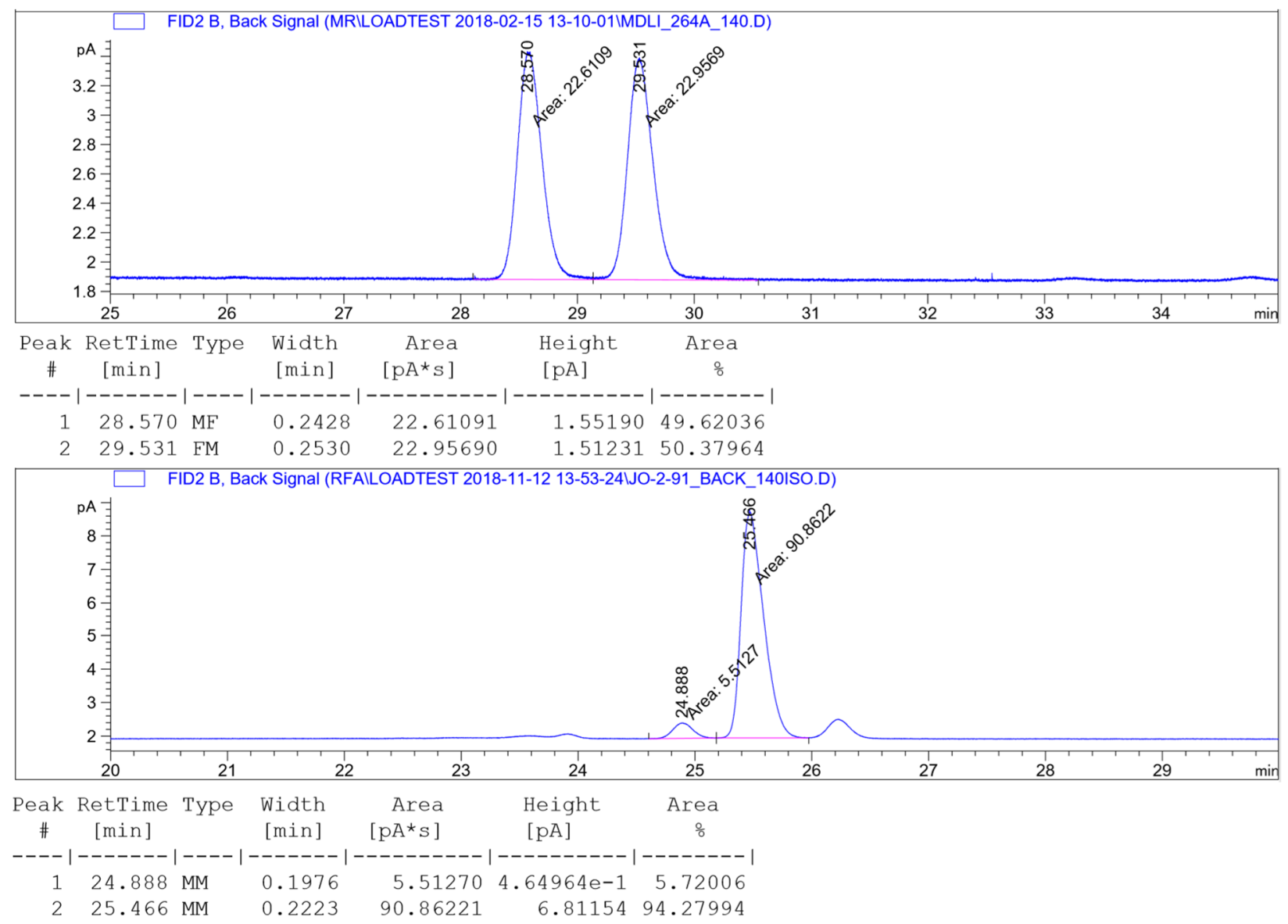

(1)

(R)-4-(2-bromo-1,1-difluorohexyl)benzonitrile. 10 was prepared from $20(52.8 \mathrm{mg}, 0.2 \mathrm{mmol})$ according to the General Procedure as a white solid ( $45.9 \mathrm{mg}, 76 \%$ yield).

${ }^{1} \mathrm{H}$ NMR $\left(500 \mathrm{MHz}, \mathrm{CDCl}_{3}\right) \delta 7.75(\mathrm{~d}, J=8.8 \mathrm{~Hz}, 2 \mathrm{H}), 7.64(\mathrm{~d}, J=8.4 \mathrm{~Hz}, 2 \mathrm{H}), 4.17$ (dddd, $J=13.7,11.4,8.8,2.7$ $\mathrm{Hz}, 1 \mathrm{H}), 2.01$ (dddd, $J=14.3,9.8,5.8,2.7 \mathrm{~Hz}, 1 \mathrm{H}), 1.79-1.58(\mathrm{~m}, 2 \mathrm{H}), 1.45-1.18(\mathrm{~m}, 3 \mathrm{H}), 0.91(\mathrm{t}, J=7.2 \mathrm{~Hz}$, $3 \mathrm{H})$.

${ }^{13} \mathrm{C}$ NMR $\left(126 \mathrm{MHz}, \mathrm{CDCl}_{3}\right) \delta 139.08(\mathrm{t}, J=27.3 \mathrm{~Hz}), 132.07,127.09(\mathrm{t}, J=6.2 \mathrm{~Hz}), 119.50(\mathrm{t}, J=248.2 \mathrm{~Hz})$, $117.89,114.47,54.55$ (t, $J=31.3 \mathrm{~Hz}), 31.18$ (t, $J=2.4 \mathrm{~Hz}), 29.37,21.82,13.78$.

${ }^{19} \mathrm{~F}$ NMR $\left(471 \mathrm{MHz}, \mathrm{CDCl}_{3}\right) \delta-96.86(\mathrm{~d}, J=247.8 \mathrm{~Hz}),-103.21(\mathrm{~d}, J=246.7 \mathrm{~Hz})$.

HRMS (EI): for $\mathrm{C}_{13} \mathrm{H}_{14} \mathrm{BrF}_{2} \mathrm{~N}$, [M] $]^{+}$calculated $\mathrm{m} / z=301.0272$ and 303.0252 , found $\mathrm{m} / z=301.0272$ and 303.0251

Chiral GC: CP-Chirasil-Dex CB, $40{ }^{\circ} \mathrm{C}$ to $200{ }^{\circ} \mathrm{C}, 1{ }^{\circ} \mathrm{C} / \mathrm{min}, 88 \%$ ee 

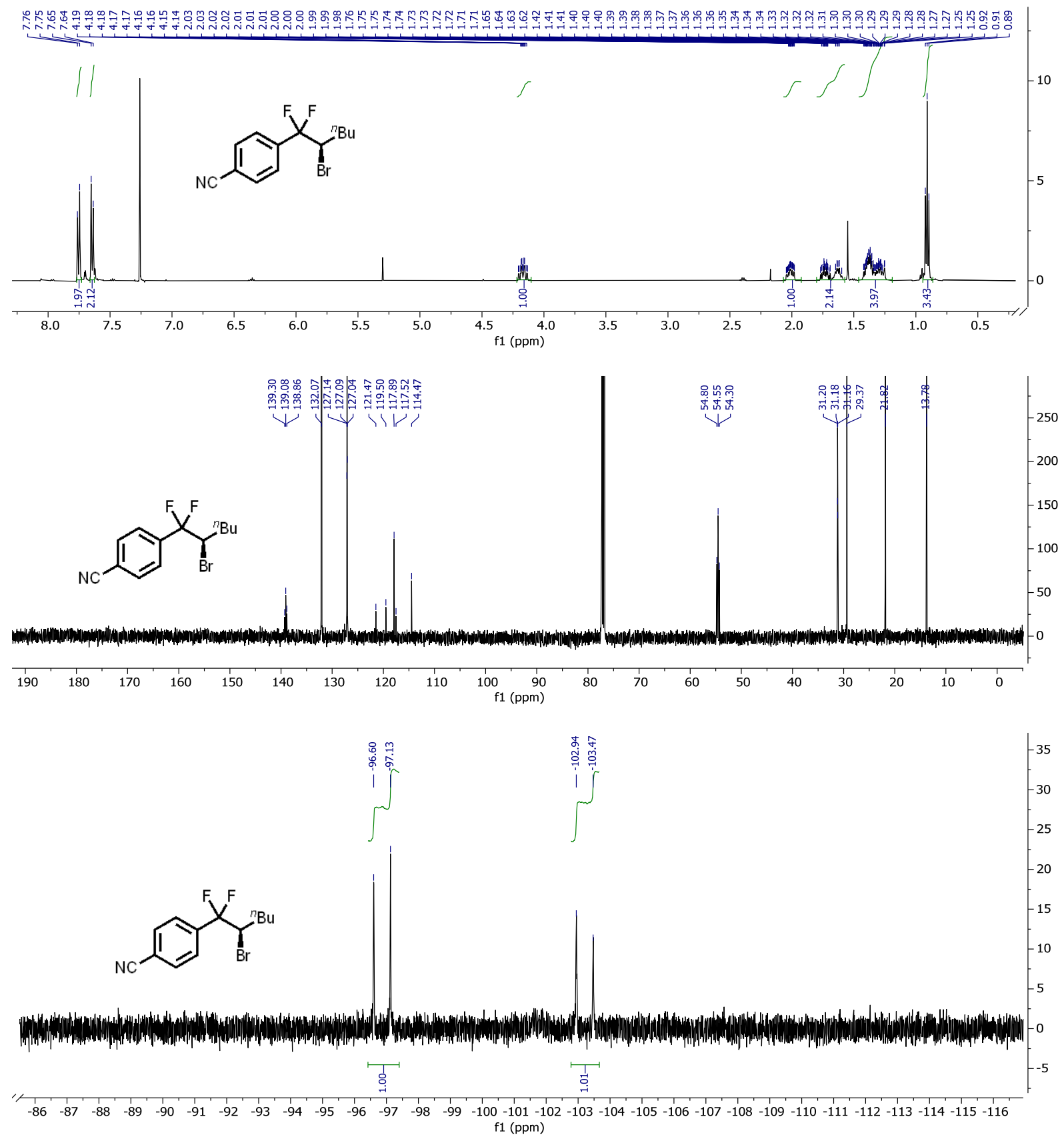

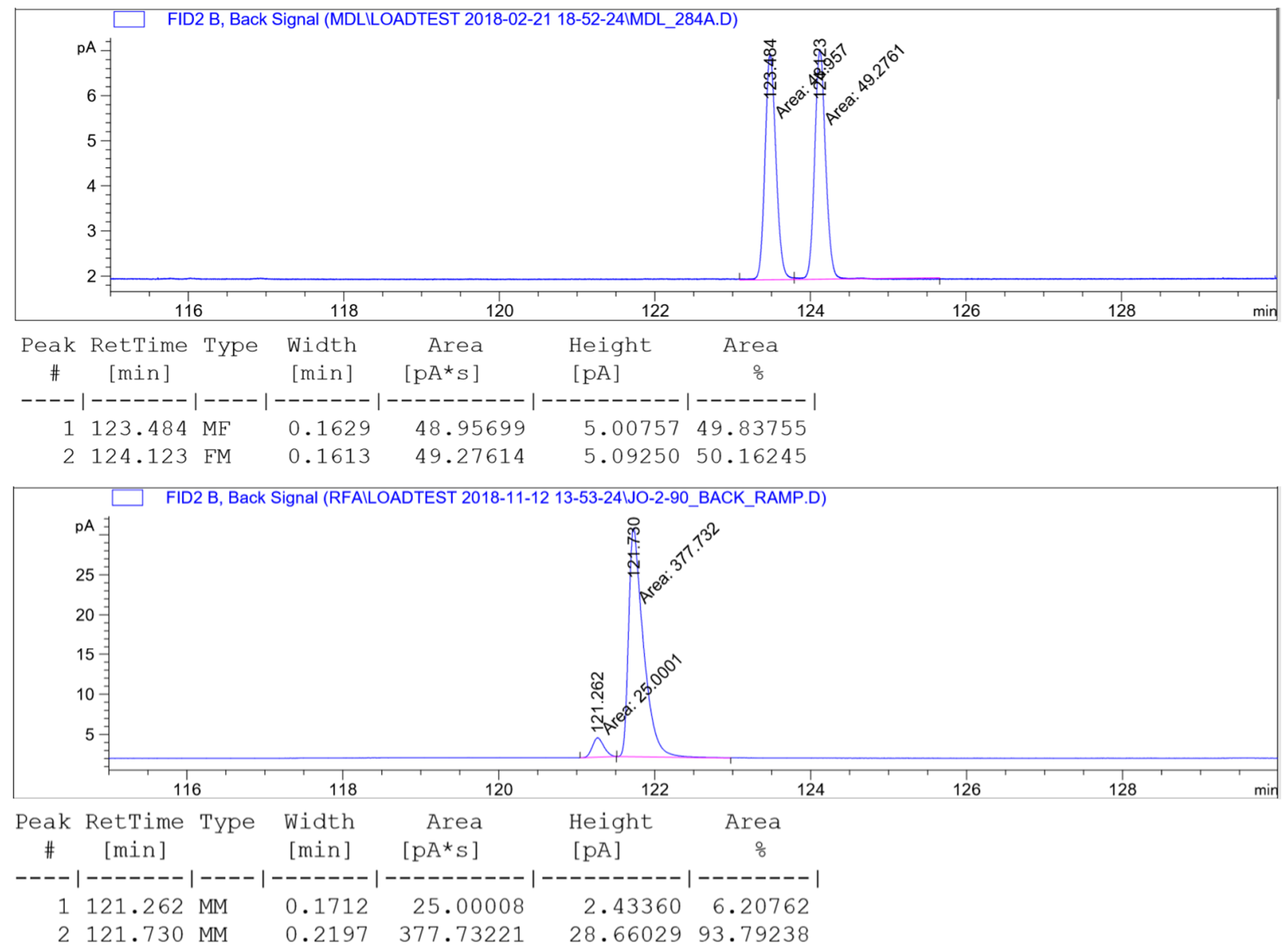

$\overbrace{\mathrm{Br}}^{\mathrm{CH}}$

(R)-1-bromo-4-(2-bromo-1,1-difluoropropyl)benzene. 1p was prepared from $2 \mathbf{p}$ ( $55.2 \mathrm{mg}, 0.2 \mathrm{mmol}$ ) according to the General Procedure as a clear, colorless oil $(50.9 \mathrm{mg}, 81 \%$ yield $)$.

${ }^{1} \mathrm{H}$ NMR $\left(500 \mathrm{MHz}, \mathrm{CDCl}_{3}\right) \delta 7.59(\mathrm{~d}, J=8.3 \mathrm{~Hz}, 2 \mathrm{H}), 7.41-7.36(\mathrm{~m}, 2 \mathrm{H}), 4.32(\mathrm{tq}, J=11.0,6.9 \mathrm{~Hz}, 1 \mathrm{H}), 1.71(\mathrm{~d}$, $J=6.9 \mathrm{~Hz}, 3 \mathrm{H})$.

${ }^{13} \mathrm{C}$ NMR $\left(126 \mathrm{MHz}, \mathrm{CDCl}_{3}\right) \delta 133.03(\mathrm{t}, J=27.1 \mathrm{~Hz}), 131.56,127.79$ (t, $\left.J=6.1 \mathrm{~Hz}\right), 124.93(\mathrm{~d}, J=2.2 \mathrm{~Hz}), 119.88$ (t, $J=247.5 \mathrm{~Hz}), 47.99(\mathrm{t}, J=33.1 \mathrm{~Hz}), 19.46(\mathrm{t}, J=3.1 \mathrm{~Hz})$.

${ }^{19} \mathrm{~F}$ NMR $\left(376 \mathrm{MHz}, \mathrm{CDCl}_{3}\right) \delta-102.30(\mathrm{dd}, J=245.5,10.9 \mathrm{~Hz}, 1 \mathrm{~F}),-103.81(\mathrm{dd}, J=245.1,11.5 \mathrm{~Hz}, 1 \mathrm{~F})$.

HRMS (EI): for $\mathrm{C}_{9} \mathrm{H}_{8} \mathrm{Br}_{2} \mathrm{~F}_{2},[\mathrm{M}]^{+}$calculated $m / z=311.8955$ and 313.8935 and 315.8914 , found $m / z=311.8954$ and 313.8932 and 315.8911

Chiral GC: CP-Chirasil-Dex CB, $40{ }^{\circ} \mathrm{C}$ to $160{ }^{\circ} \mathrm{C}, 0.5{ }^{\circ} \mathrm{C} / \mathrm{min}, 7 \mathrm{psi}, 82 \%$ ee 

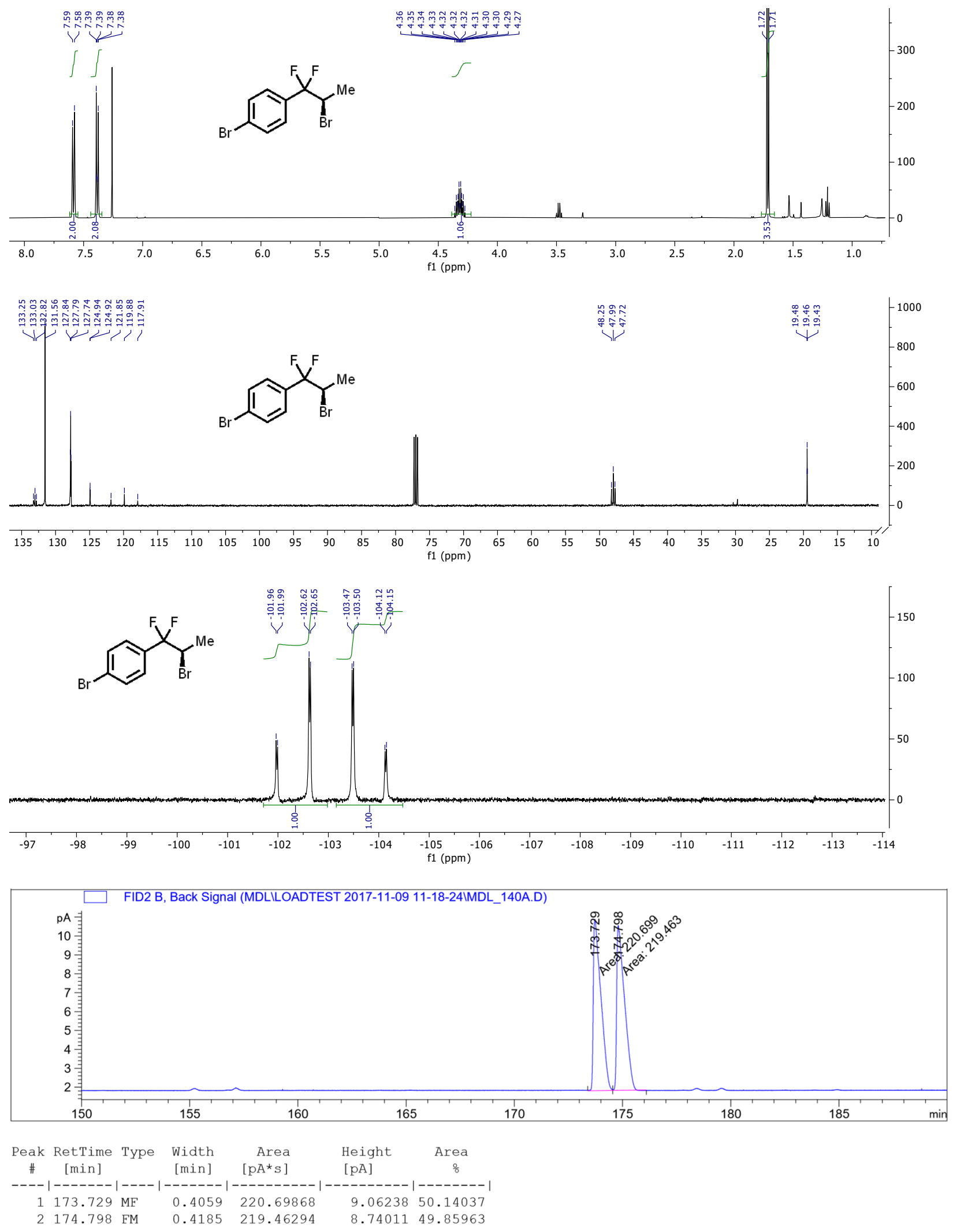


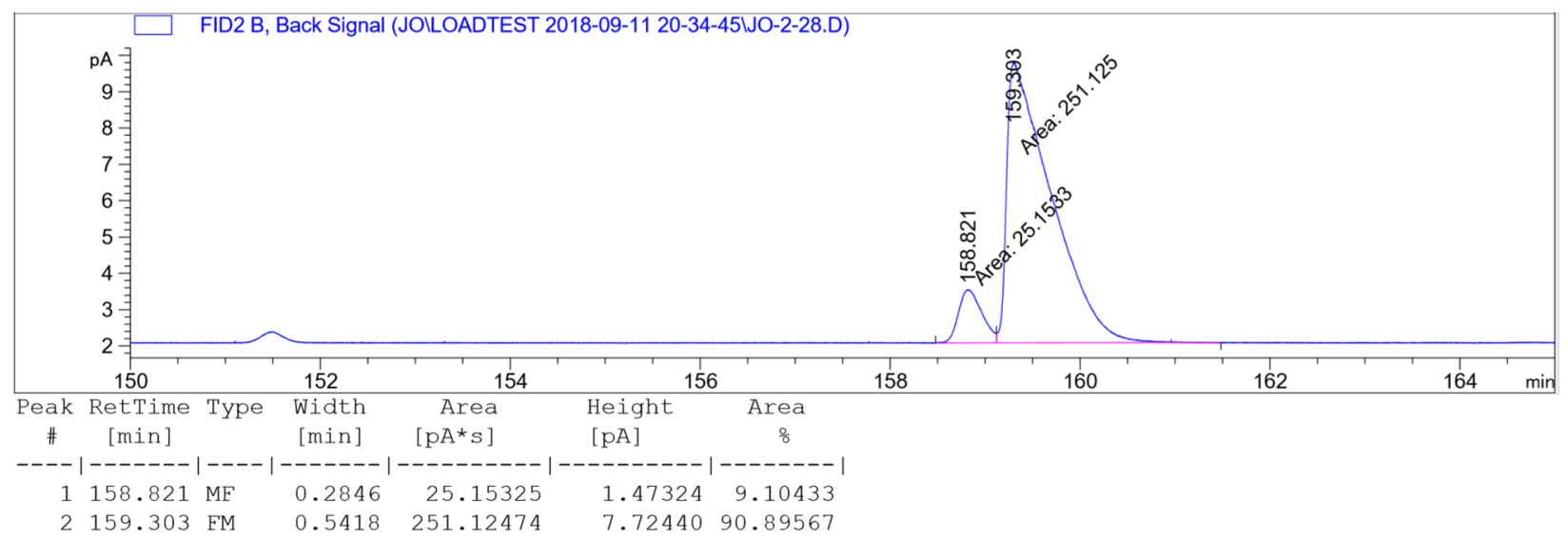<smiles>CC(Br)C(F)(F)c1cccc(Cl)c1</smiles>

(R)-1-(2-bromo-1,1-difluoropropyl)-3-chlorobenzene. 1q was prepared from $2 \mathbf{2 q}(46.3 \mathrm{mg}, 0.2 \mathrm{mmol}$ ) according to the General Procedure as a clear, colorless oil ( $38.3 \mathrm{mg}, 71 \%$ yield).

${ }^{1} \mathrm{H}$ NMR $\left(500 \mathrm{MHz}, \mathrm{CDCl}_{3}\right) \delta 7.51(\mathrm{t}, J=0.6 \mathrm{~Hz}, 1 \mathrm{H}), 7.48-7.43(\mathrm{~m}, 1 \mathrm{H}), 7.42-7.37$ (m, 2H), 4.32 (tq, $J=11.3$, $7.0 \mathrm{~Hz}, 1 \mathrm{H}), 1.72(\mathrm{~d}, J=7.0 \mathrm{~Hz}, 3 \mathrm{H})$.

${ }^{13} \mathrm{C}$ NMR $\left(126 \mathrm{MHz}, \mathrm{CDCl}_{3}\right) \delta 135.93(\mathrm{t}, J=27.1 \mathrm{~Hz}), 134.49,130.60,129.67,126.39(\mathrm{t}, J=6.5 \mathrm{~Hz}), 124.30(\mathrm{t}, J=$ $6.1 \mathrm{~Hz}), 119.45(\mathrm{t}, J=247.7 \mathrm{~Hz}), 47.89(\mathrm{t}, J=32.7 \mathrm{~Hz}), 19.46$.

${ }^{19} \mathrm{~F}$ NMR $\left(471 \mathrm{MHz}, \mathrm{CDCl}_{3}\right) \delta-103.22(\mathrm{dd}, J=245.3,11.0 \mathrm{~Hz}, 1 \mathrm{~F}),-104.31(\mathrm{dd}, J=245.1,11.5 \mathrm{~Hz}, 1 \mathrm{~F})$.

HRMS (EI): for $\mathrm{C}_{9} \mathrm{H}_{8} \mathrm{BrClF}_{2}$, [M] $]^{+}$calculated $\mathrm{m} / \mathrm{z}=267.9460$ and 269.9440 , found $\mathrm{m} / z=267.9459$ and 269.9434 .

Chiral GC: $\beta$-Cyclosil, $40{ }^{\circ} \mathrm{C}$ to $140{ }^{\circ} \mathrm{C}, 0.5^{\circ} \mathrm{C} / \mathrm{min}, 7 \mathrm{psi}, 79 \%$ ee
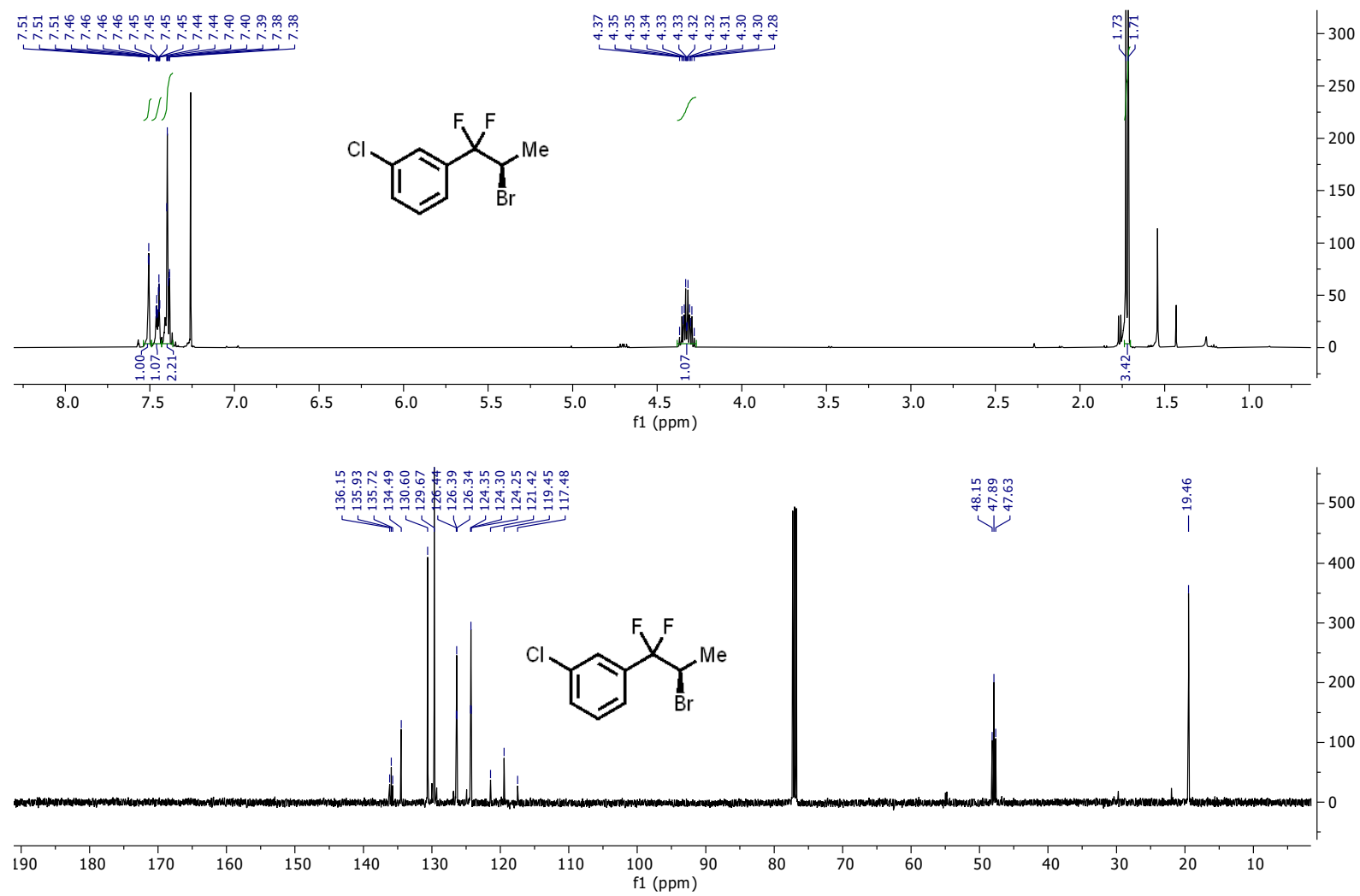

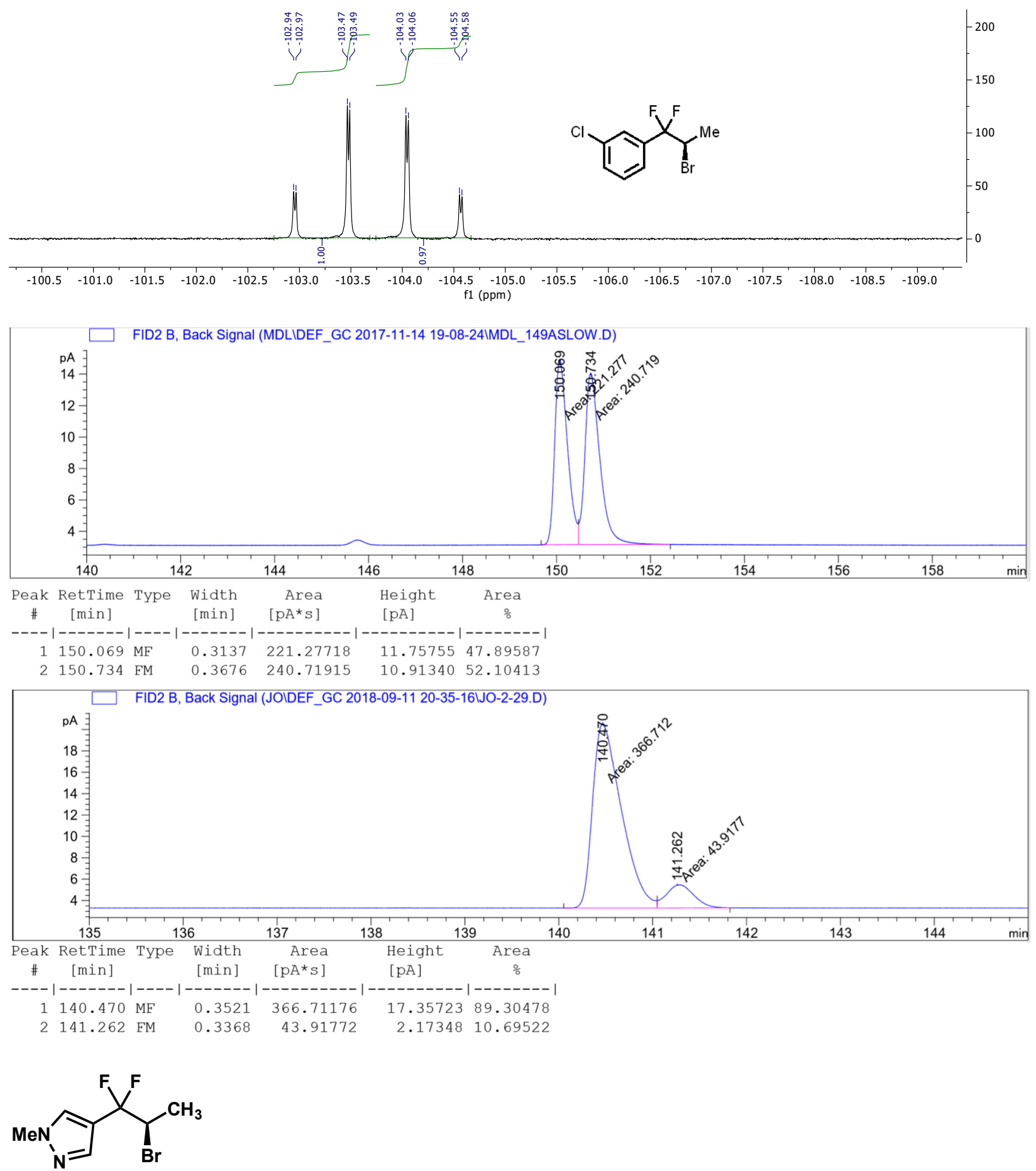

(R)-4-(2-bromo-1,1-difluoropropyl)-1-methyl-1H-pyrazole. 1r was prepared from $2 \mathbf{r}$ (40.2 $\mathrm{mg}, 0.2 \mathrm{mmol}$ ) according to the General Procedure as a clear, colorless oil $(39.2 \mathrm{mg}, 82 \%$ yield $)$.

${ }^{1} \mathrm{H}$ NMR $\left(500 \mathrm{MHz}, \mathrm{CDCl}_{3}\right) \delta 7.60(\mathrm{~s}, 1 \mathrm{H}), 7.57(\mathrm{~s}, 1 \mathrm{H}), 4.39-4.24(\mathrm{~m}, 1 \mathrm{H}), 3.94(\mathrm{~s}, 3 \mathrm{H}), 1.71(\mathrm{~d}, J=7.0 \mathrm{~Hz}, 3 \mathrm{H})$. ${ }^{13} \mathrm{C}$ NMR $\left(126 \mathrm{MHz}, \mathrm{CDCl}_{3}\right) \delta 137.55,129.52(\mathrm{t}, J=5.4 \mathrm{~Hz}), 118.71(\mathrm{t}, J=241.9 \mathrm{~Hz}), 116.20(\mathrm{t}, J=31.7 \mathrm{~Hz})$, $48.36(\mathrm{t}, J=34.1 \mathrm{~Hz}), 39.24,19.62$.

${ }^{19} \mathrm{~F}$ NMR (471 MHz, CDCl3) $\delta-93.66$ (dd, $\left.J=253.8,6.3 \mathrm{~Hz}, 1 \mathrm{~F}\right),-96.41$ (dd, $\left.J=254.8,10.0 \mathrm{~Hz}, 1 \mathrm{~F}\right)$.

HRMS (ESI): for $\mathrm{C}_{7} \mathrm{H}_{10} \mathrm{BrF}_{2} \mathrm{~N}_{2},[\mathrm{M}+\mathrm{H}]^{+}$calculated $\mathrm{m} / z=238.9990$ and 240.9969 , found $\mathrm{m} / z=238.9989$ and 240.9965.

Chiral GC: CP-Chirasil-Dex CB, isothermal $110{ }^{\circ} \mathrm{C}, 7 \mathrm{psi}, 63 \%$ ee 

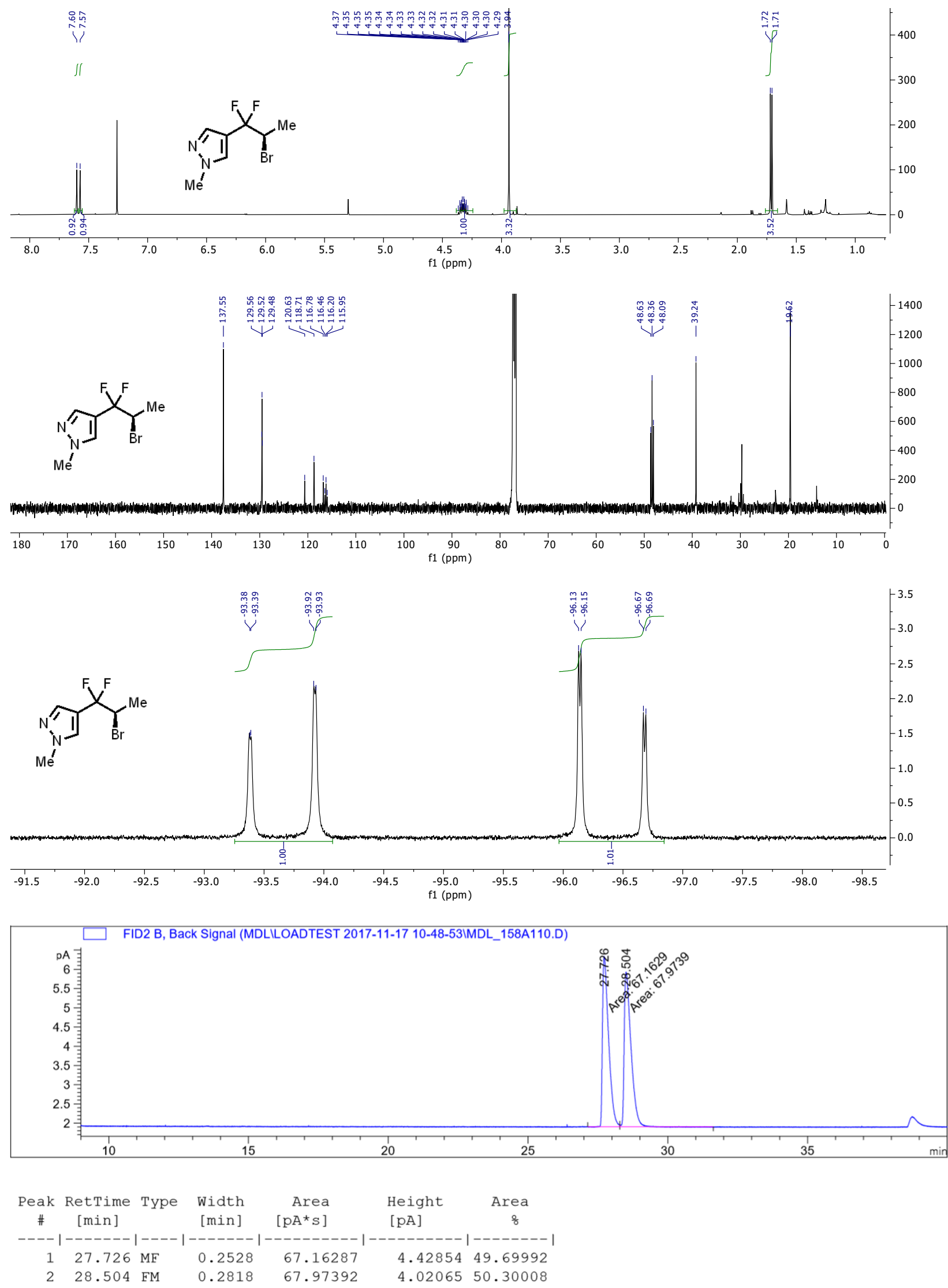


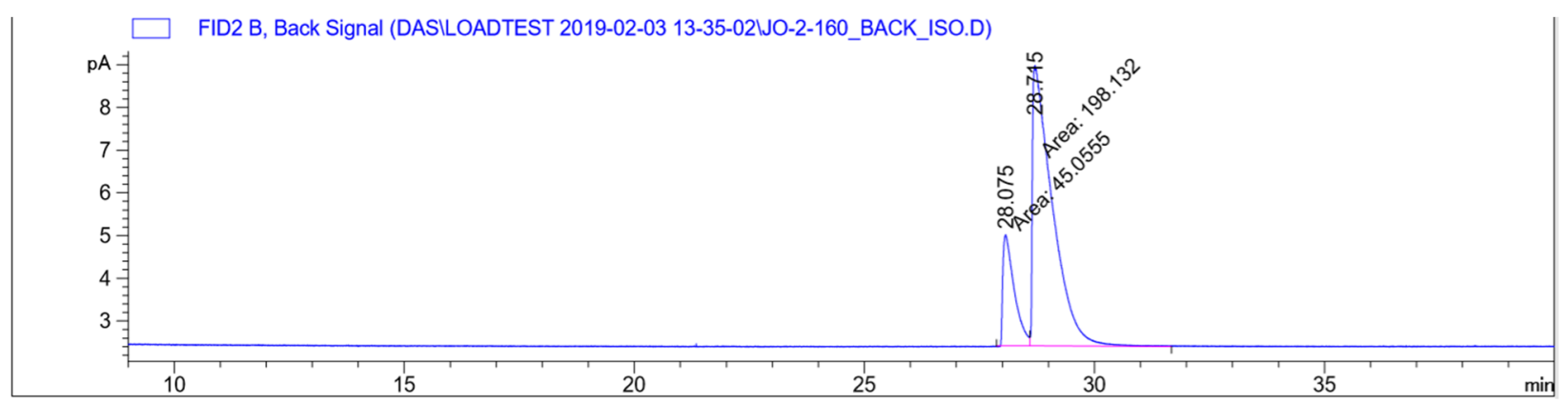

\begin{tabular}{|c|c|c|c|c|c|c|}
\hline $\begin{array}{c}\text { Peak } \\
\#\end{array}$ & $\begin{array}{c}\text { RetTime } \\
\text { [min] }\end{array}$ & Type & $\begin{array}{l}\text { Width } \\
\text { [min] }\end{array}$ & $\begin{array}{r}\text { Area } \\
{\left[\mathrm{pA} A^{*} \mathrm{~s}\right]}\end{array}$ & $\begin{array}{l}\text { Height } \\
{[\mathrm{pA}]}\end{array}$ & $\begin{array}{c}\text { Area } \\
\%\end{array}$ \\
\hline---1 & ----- & & ------ & ---------- & --------- & $--------\mid$ \\
\hline 1 & 28.075 & MF & 0.2886 & 45.05552 & 2.60222 & 18.52708 \\
\hline 2 & 28.715 & FM & 0.5021 & 198.13184 & 6.57730 & 81.47292 \\
\hline
\end{tabular}

(

(R)-2-bromo-3,3-difluoro-3-(4-nitrophenyl)propan-1-ol. 1s was prepared from $2 \mathbf{s}$ (51.6 $\mathrm{mg}, 0.2 \mathrm{mmol}$ ) according to the General Procedure as a clear, colorless oil ( $44.4 \mathrm{mg}, 75 \%$ yield).

${ }^{1} \mathrm{H}$ NMR $\left(399 \mathrm{MHz}, \mathrm{CDCl}_{3}\right) \delta 8.33(\mathrm{~d}, J=9.1 \mathrm{~Hz}, 1 \mathrm{H}), 7.73(\mathrm{~d}, J=8.8 \mathrm{~Hz}, 2 \mathrm{H}), 4.40(\mathrm{dddd}, J=14.8,8.9,7.3,4.1$ $\mathrm{Hz}, 1 \mathrm{H}), 4.19-4.03(\mathrm{~m}, 1 \mathrm{H}), 3.99-3.83(\mathrm{~m}, 1 \mathrm{H}), 1.98(\mathrm{~s}, 1 \mathrm{H})$.

${ }^{13} \mathrm{C}$ NMR $\left(126 \mathrm{MHz}, \mathrm{CDCl}_{3}\right) \delta 149.23,140.18(\mathrm{t}, J=26.7 \mathrm{~Hz}), 127.33(\mathrm{t}, J=6.2 \mathrm{~Hz}), 123.65,119.01(\mathrm{t}, J=249.9$, $248.6 \mathrm{~Hz}), 62.23(\mathrm{t}, J=3.2 \mathrm{~Hz}), 56.99-52.11(\mathrm{~m})$.

${ }^{19} \mathrm{~F}$ NMR $\left(471 \mathrm{MHz}, \mathrm{CDCl}_{3}\right) \delta-95.15(\mathrm{~d}, J=252.0 \mathrm{~Hz}),-101.92(\mathrm{~d}, J=252.2 \mathrm{~Hz})$.

HRMS (ESI): for $\mathrm{C}_{9} \mathrm{H}_{9} \mathrm{BrF}_{2} \mathrm{NO}_{3},[\mathrm{M}+\mathrm{H}]^{+}$calculated $\mathrm{m} / \mathrm{z}=293.9583$ and 295.9562 , found $\mathrm{m} / \mathrm{z}=293.9583$ and 295.9560.

Chiral HPLC: Chiralpak IA, $8.0 \%$ isopropanol/hexanes, $1.0 \mathrm{ml} / \mathrm{min}, 90 \%$ ee

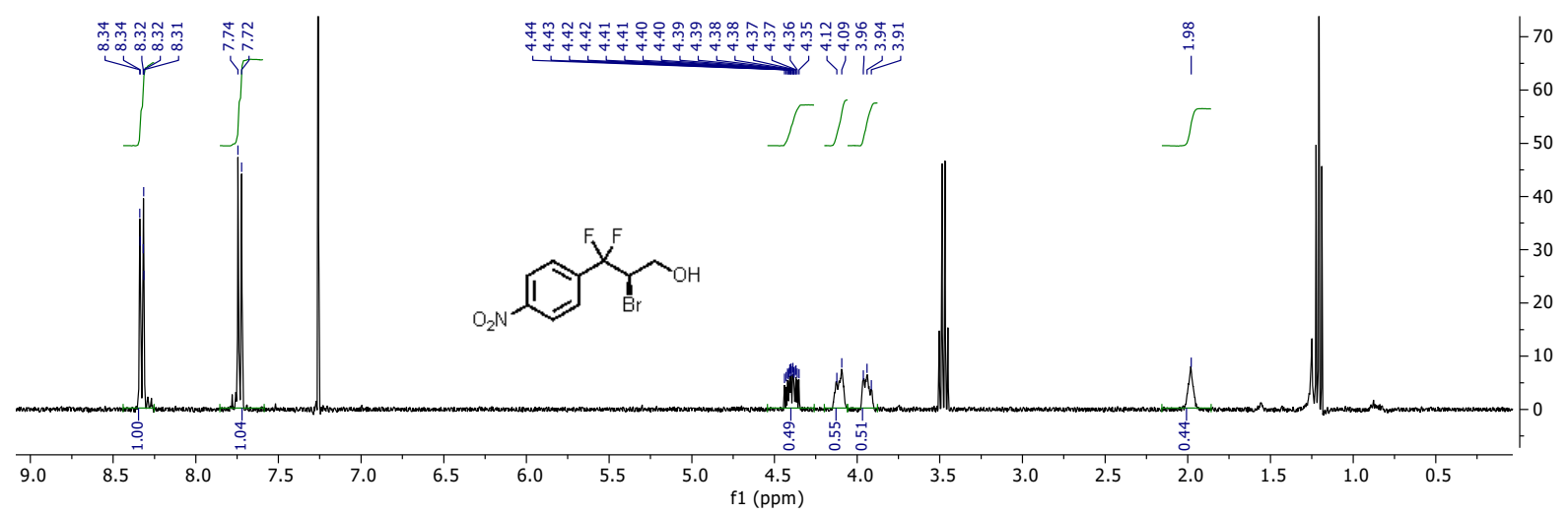



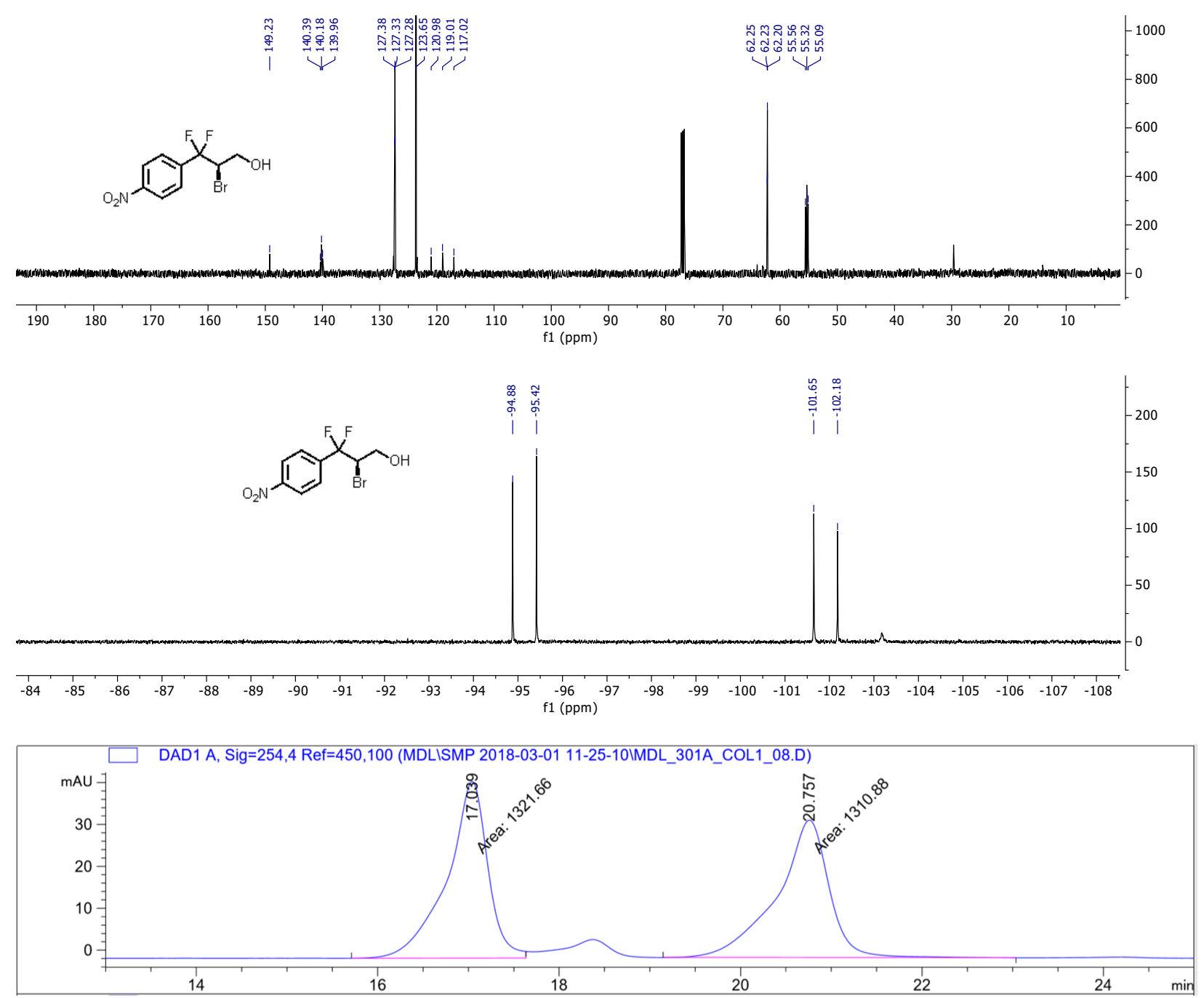

Signal 1: DAD1 A, Sig=254, 4 Ref $=450,100$
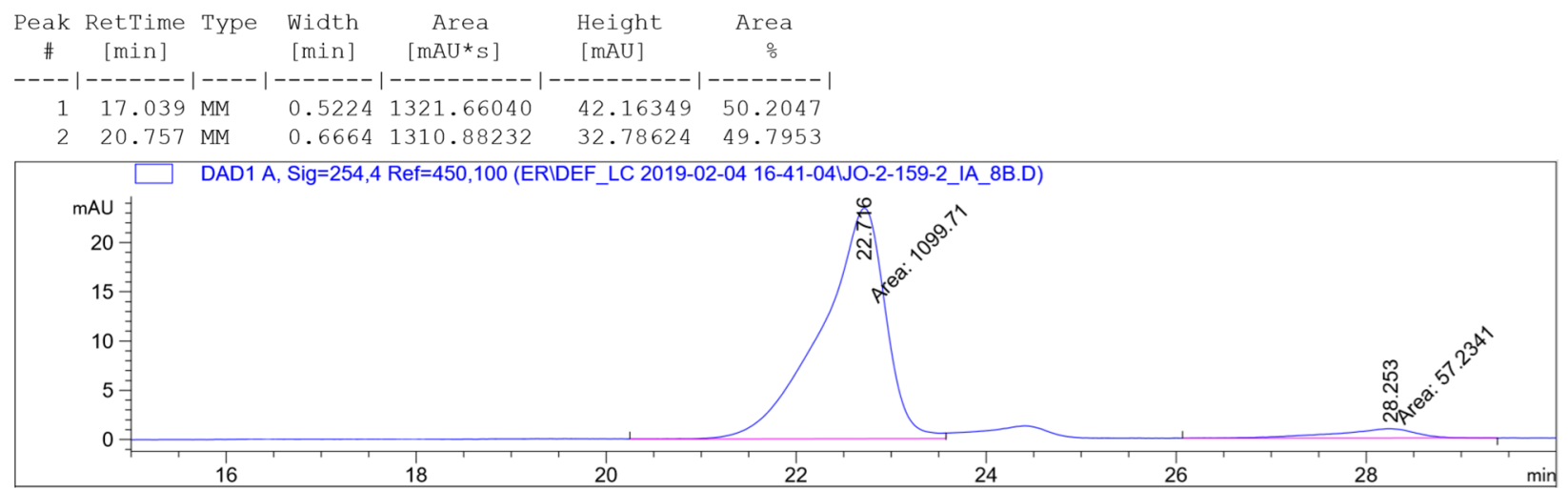

Signal 1: DAD1 A, Sig=254, 4 Ref $=450,100$

\begin{tabular}{|c|c|c|c|c|c|c|}
\hline $\begin{array}{c}\text { Peak } \\
\#\end{array}$ & $\begin{array}{c}\text { RetTime } \\
\text { [min] }\end{array}$ & Type & $\begin{array}{c}\text { Width } \\
\text { [min] }\end{array}$ & $\begin{array}{c}\text { Area } \\
{\left[\mathrm{mAU}{ }^{*} \mathrm{~s}\right]}\end{array}$ & $\begin{array}{l}\text { Height } \\
{[\mathrm{mAU}]}\end{array}$ & $\begin{array}{c}\text { Area } \\
\frac{\circ}{6}\end{array}$ \\
\hline--- & $\mid--$ & & ----1 & ---------- & --------- & $-------\mid$ \\
\hline 1 & 22.716 & MM & 0.7829 & 1099.70837 & 23.41049 & 95.0530 \\
\hline 2 & 28.253 & $\mathrm{MM}$ & 0.9952 & 57.23410 & $9.58472 e-1$ & 4.9470 \\
\hline
\end{tabular}


<smiles>FC(F)(c1cccc(Br)c1)[C@H](Br)CCBr</smiles>

(R)-1-bromo-3-(2,4-dibromo-1,1-difluorobutyl)benzene. $\mathbf{1 t}$ was prepared from $\mathbf{2 t}$ (73.8 $\mathrm{mg}, 0.2 \mathrm{mmol}$ ) according to the General Procedure as a clear, colorless oil ( $79.8 \mathrm{mg}, 98 \%$ yield). Enantiomeric excess was determined from the thioacetate substitution product. See S2 below.

${ }^{1} \mathrm{H}$ NMR $\left(500 \mathrm{MHz}, \mathrm{CDCl}_{3}\right) \delta 7.69(\mathrm{~s}, 1 \mathrm{H}), 7.64(\mathrm{~d}, J=7.7 \mathrm{~Hz}, 1 \mathrm{H}), 7.47(\mathrm{~d}, J=7.8 \mathrm{~Hz}, 1 \mathrm{H}), 7.36(\mathrm{t}, J=7.9 \mathrm{~Hz}$, $1 \mathrm{H}), 4.50(\mathrm{dtd}, J=13.3,10.7,2.7 \mathrm{~Hz}, 1 \mathrm{H}), 3.66(\mathrm{ddd}, J=10.0,5.8,3.9 \mathrm{~Hz}, 1 \mathrm{H}), 3.58(\mathrm{td}, J=10.3,4.7 \mathrm{~Hz}, 1 \mathrm{H}), 2.47$ (dddd, $J=15.7,10.4,5.8,2.7 \mathrm{~Hz}, 1 \mathrm{H}), 2.34$ (ddq, $J=15.1,11.0,3.9 \mathrm{~Hz}, 1 \mathrm{H}$ ).

${ }^{13} \mathrm{C}$ NMR $\left(126 \mathrm{MHz}, \mathrm{CDCl}_{3}\right) \delta 136.12(\mathrm{t}, J=26.6 \mathrm{~Hz}), 133.78,130.08,129.11(\mathrm{t}, J=6.4 \mathrm{~Hz}), 124.66(\mathrm{t}, J=6.1 \mathrm{~Hz})$, $122.59,119.15(\mathrm{t}, J=249.8,247.7 \mathrm{~Hz}), 53.80-50.59(\mathrm{~m}), 34.37(\mathrm{t}, J=2.6 \mathrm{~Hz}), 30.26$.

${ }^{19} \mathrm{~F}$ NMR $\left(471 \mathrm{MHz}, \mathrm{c}_{6} \mathrm{~d}_{6}\right) \delta-97.98(\mathrm{dd}, J=246.8,10.3 \mathrm{~Hz}),-101.04(\mathrm{dd}, J=246.5,13.3 \mathrm{~Hz})$.

HRMS (EI): for $\mathrm{C}_{10} \mathrm{H}_{9} \mathrm{Br}_{3} \mathrm{~F}_{2},[\mathrm{M}]^{+}$calculated $\mathrm{m} / \mathrm{z}=403.8217$ and 405.8196 and 407.8176 and 409.8156 , found $\mathrm{m} / \mathrm{z}=$ 403.8211 and 405.8190 and 407.8169 and 409.8149 .

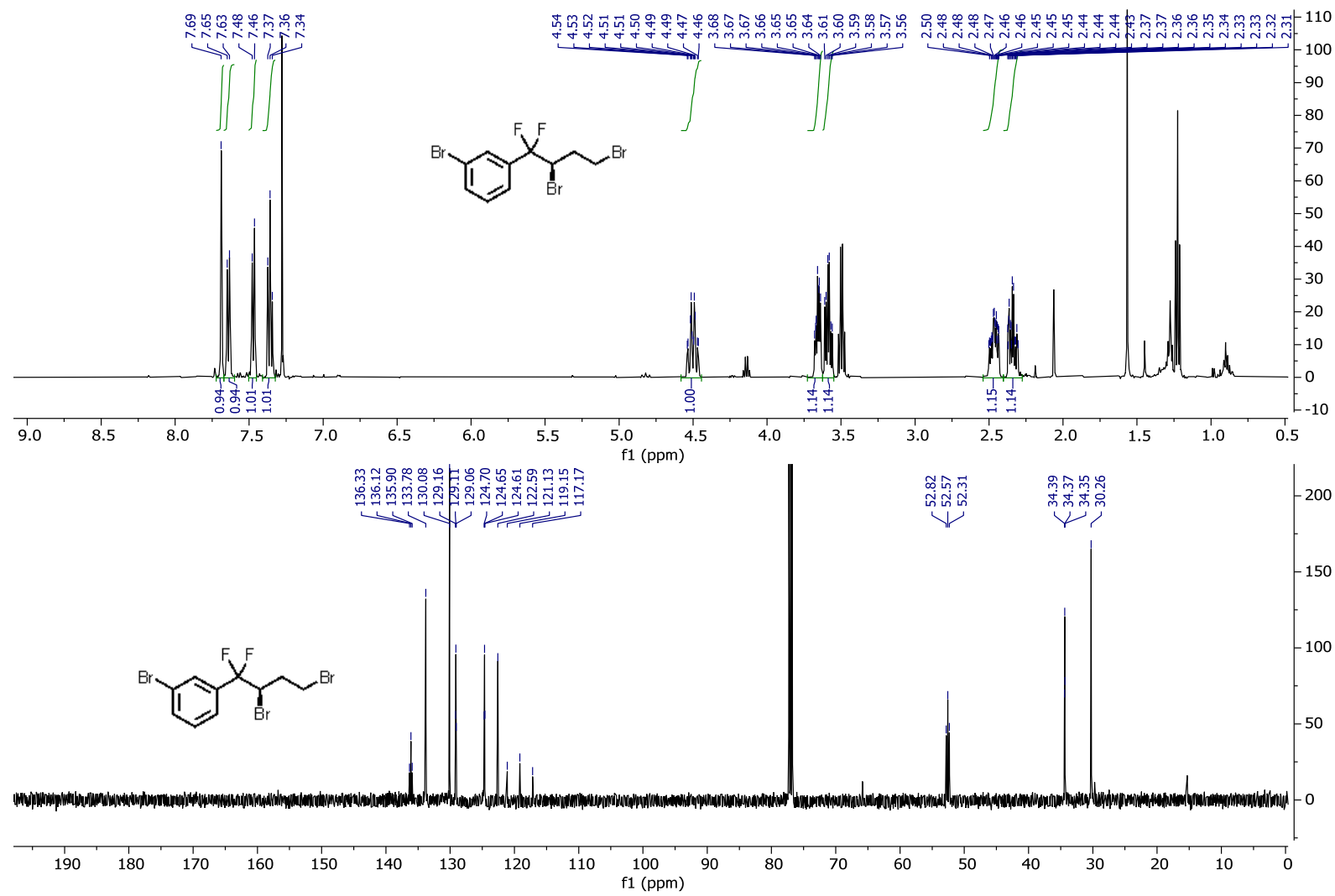




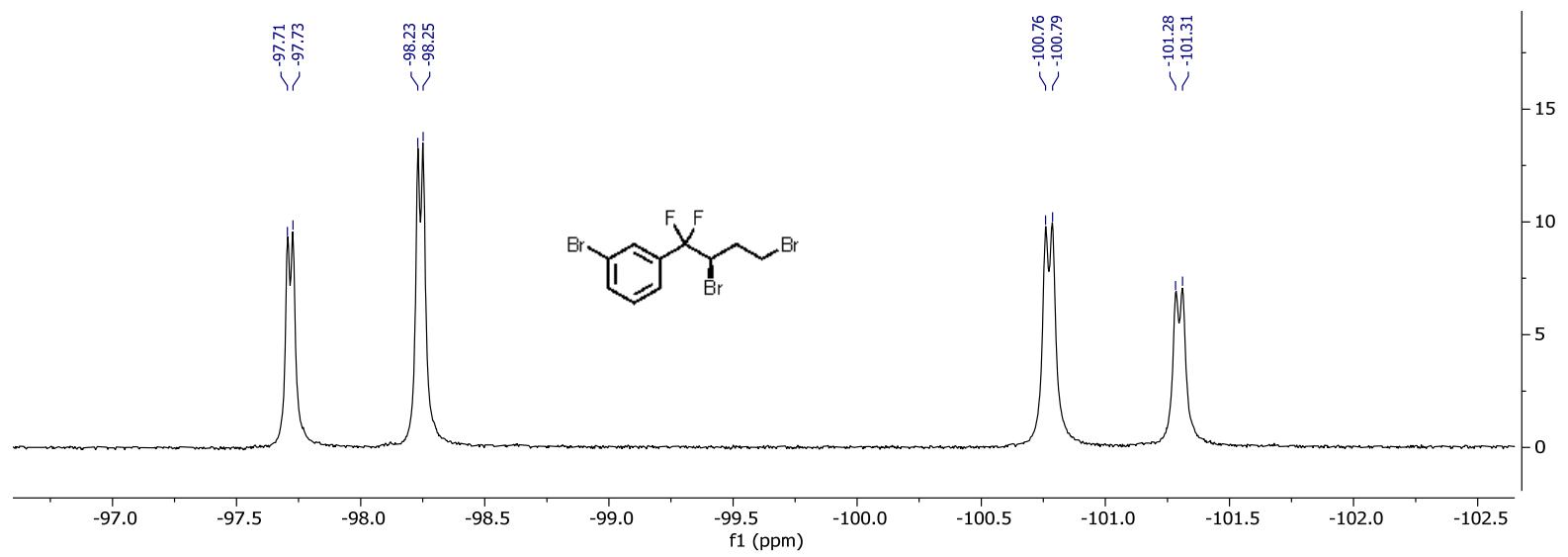




\section{Derivatizations}<smiles>C[C@H](Br)C(F)(F)c1cccc([N+](=O)[O-])c1</smiles><smiles>C[C@@H](N)C(F)(F)c1cccc([N+](=O)[O-])c1</smiles>

5. (S)-1-(2-azido-1,1-difluoropropyl)-3-nitrobenzene. To a solution of 1a (28 mg, $0.1 \mathrm{mmol})$ in DMSO (0.3 mL) under $\mathrm{N}_{2}$, was added solid $\mathrm{NaN}_{3}$ (20 mg, 3 equiv). The mixture was briefly degassed by direct exposure to high vacuum, refilled with $\mathrm{N}_{2}$, and sealed. The mixture was heated at $100^{\circ} \mathrm{C}$ for 12 hours, and upon cooling was diluted with brine. The aqueous layer was extracted $3 \mathrm{x}$ with $\mathrm{Et}_{2} \mathrm{O}$, and the combined organic layers were washed $3 \mathrm{x}$ with brine, dried over $\mathrm{MgSO}_{4}$, filtered, and concentrated. The crude residue was purified by column chromatography eluting with Hexanes/Et $2 \mathrm{O}$ on a gradient from pure hexanes to $5 \% \mathrm{Et}_{2} \mathrm{O}$, affording the title compound as a colorless oil (18 mg, 74\%).

${ }^{1} \mathrm{H}$ NMR $\left(500 \mathrm{MHz}, \mathrm{CDCl}_{3}\right) \delta 8.40-8.33(\mathrm{~m}, 1 \mathrm{H}), 7.88-7.82(\mathrm{~m}, 0 \mathrm{H}), 7.68(\mathrm{t}, J=7.9 \mathrm{~Hz}, 0 \mathrm{H}), 3.99-3.87(\mathrm{~m}$, $0 \mathrm{H}), 1.38(\mathrm{~d}, J=6.8 \mathrm{~Hz}, 1 \mathrm{H})$.

${ }^{13} \mathrm{C} \mathrm{NMR}\left(126 \mathrm{MHz} \mathrm{CDCl}_{3}\right) \delta 148.19,135.86(\mathrm{t}, J=27.3 \mathrm{~Hz}), 131.96(\mathrm{t}, J=6.0 \mathrm{~Hz}), 129.74,125.34,121.37(\mathrm{t}, J=$ $6.8 \mathrm{~Hz}), 120.22(\mathrm{~d}, J=249.7 \mathrm{~Hz}), 60.91(\mathrm{t}, J=30.8 \mathrm{~Hz}), 13.06(\mathrm{t}, J=3.2 \mathrm{~Hz})$.

${ }^{19} \mathrm{~F}$ NMR $\left(471 \mathrm{MHz}, \mathrm{CDCl}_{3}\right) \delta-101.13(\mathrm{dd}, J=252.3,7.8 \mathrm{~Hz}, 1 \mathrm{~F}),-108.82(\mathrm{dd}, J=252.5,12.7 \mathrm{~Hz}, 1 \mathrm{~F})$.

HRMS (ESI): for $\mathrm{C}_{9} \mathrm{H}_{8} \mathrm{~F}_{2} \mathrm{O}_{2} \mathrm{Na},[\mathrm{M}+\mathrm{Na}]^{+}$calculated $\mathrm{m} / z=265.0508$, found $\mathrm{m} / z=265.0508$.

Chiral GC: CP-Chirasil-Dex CB, isothermal $110^{\circ} \mathrm{C}, 14 \mathrm{psi}, 90 \%$ ee
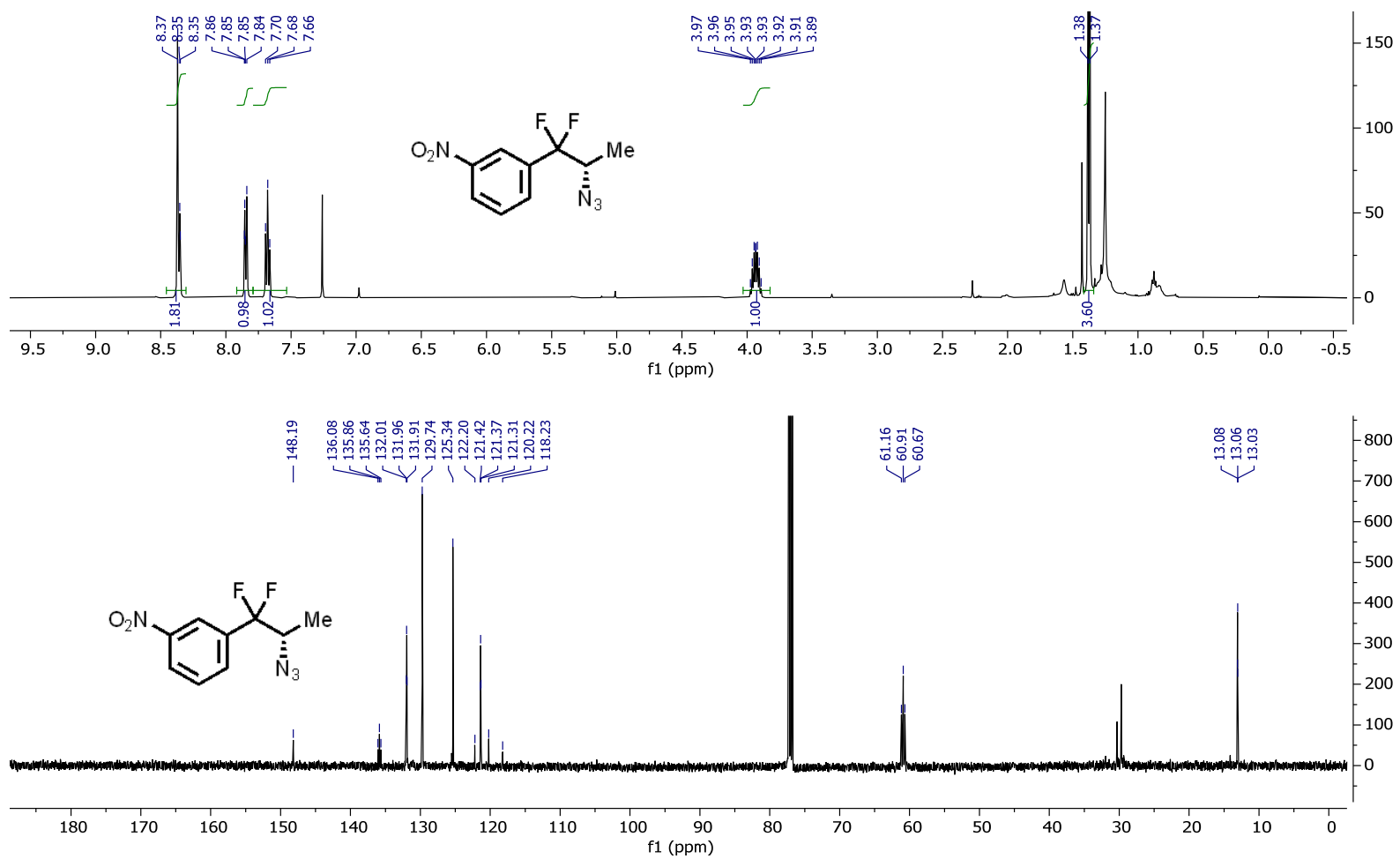

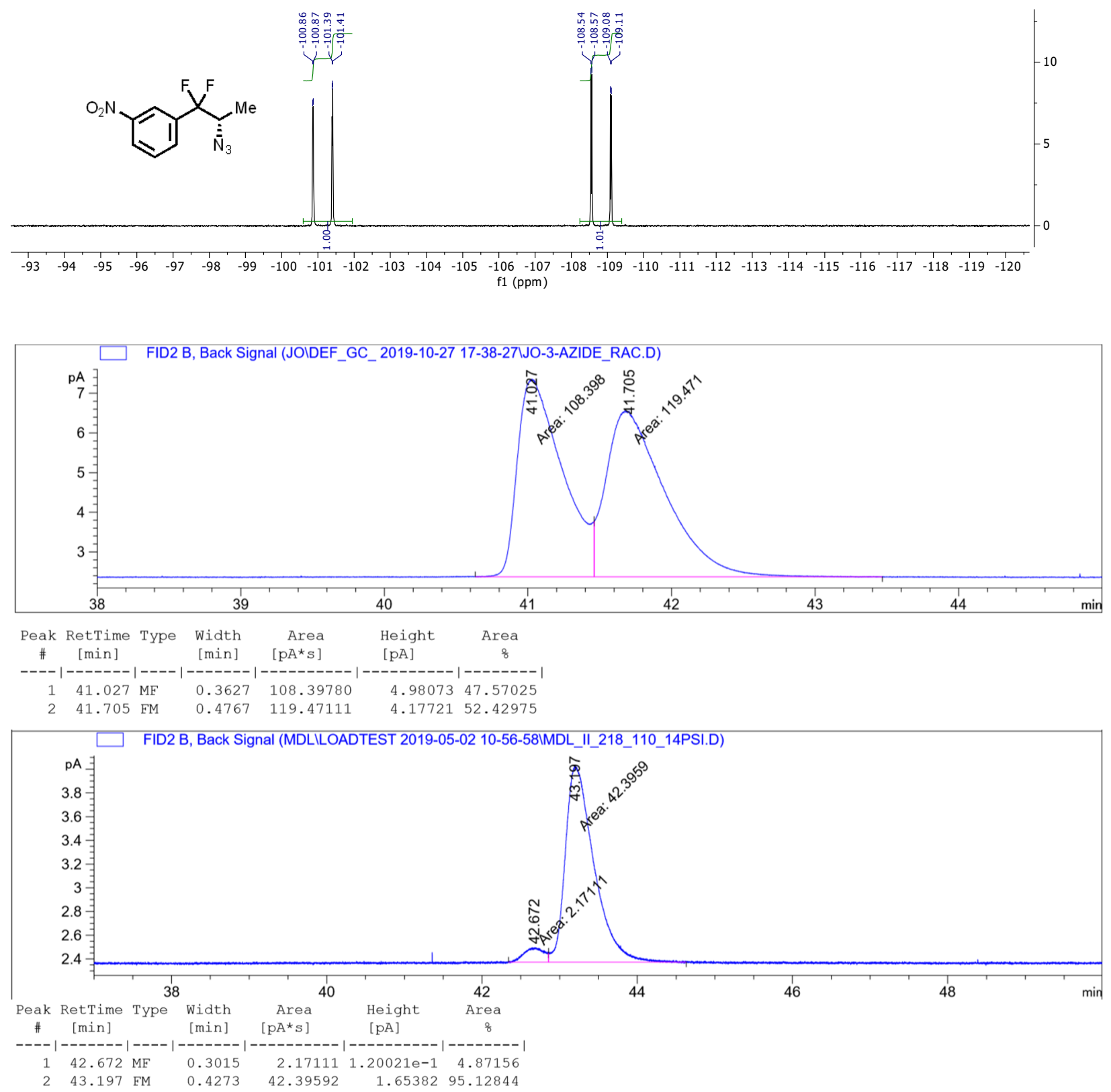

S2 (R)-S-(3-bromo-4-(3-bromophenyl)-4,4-difluorobutyl) ethanethioate. To a solution of $1 \mathbf{t}$ (82 $\mathrm{mg}, 0.2 \mathrm{mmol}$ ) in DMSO ( $2 \mathrm{~mL}$ ) under $\mathrm{N}_{2}$, was added solid KSAc ( $46 \mathrm{mg}$, 2 equiv). The mixture was briefly degassed by direct exposure to high vacuum, refilled with $\mathrm{N}_{2}$, and sealed. The mixture was stirred at room temperature for 1 hour, and upon cooling was diluted with brine. The aqueous layer was extracted $3 \mathrm{x}$ with $\mathrm{Et}_{2} \mathrm{O}$, and the combined organic layers were washed $3 \mathrm{x}$ with brine, dried over $\mathrm{MgSO}_{4}$, filtered, and concentrated. The product was used without further purification (80 $\mathrm{mg}, 77 \%)$.

${ }^{1} \mathrm{H}$ NMR (500 MHz, Chloroform- $d$ ) $\delta 7.64(\mathrm{~s}, 1 \mathrm{H}), 7.59(\mathrm{~d}, J=8.0 \mathrm{~Hz}, 1 \mathrm{H}), 7.43(\mathrm{~d}, J=7.9 \mathrm{~Hz}, 1 \mathrm{H}), 7.31$ (t, $J=7.9$ $\mathrm{Hz}, 1 \mathrm{H}), 4.28(\mathrm{td}, J=13.3,12.0,9.2 \mathrm{~Hz}, 1 \mathrm{H}), 3.21(\mathrm{ddd}, J=13.0,7.8,4.4 \mathrm{~Hz}, 1 \mathrm{H}), 2.92(\mathrm{dt}, J=13.9,8.0 \mathrm{~Hz}, 1 \mathrm{H})$, 2.32 (s, 3H), 2.23 (dtd, $J=15.7,8.9,7.9,2.4 \mathrm{~Hz}, 1 \mathrm{H}), 2.05$ (dddd, $J=15.2,11.7,7.7,4.4 \mathrm{~Hz}, 1 \mathrm{H})$. 
${ }^{13} \mathrm{C}$ NMR (126 MHz, Chloroform- $d$ ) $\delta$ 194.99, $136.13(\mathrm{t}, J=26.9 \mathrm{~Hz}), 133.68,129.99,129.17(\mathrm{t}, J=6.4 \mathrm{~Hz})$, $124.77(\mathrm{t}, J=6.2 \mathrm{~Hz}), 122.50,119.17(\mathrm{t}, J=248.4 \mathrm{~Hz}), 53.19(\mathrm{t}, J=31.9 \mathrm{~Hz}), 31.72(\mathrm{t}, J=2.4 \mathrm{~Hz}), 30.66,26.97$. ${ }^{19} \mathrm{~F}$ NMR (471 MHz, Chloroform- $d$ ) $\delta-97.39$ (dd, $\left.J=246.7,10.0 \mathrm{~Hz}, 1 \mathrm{~F}\right),-100.81$ (dd, $J=246.5,12.7 \mathrm{~Hz}, 1 \mathrm{~F}$ ). HRMS (EI): for $\mathrm{C}_{12} \mathrm{H}_{12} \mathrm{Br}_{2} \mathrm{~F}_{2} \mathrm{OS}$, [M] $]^{+}$calculated $\mathrm{m} / z=399.8938$ and 401.8918 and 403.8897 , found $\mathrm{m} / z=399.8937$ and 401.8915 and 403.8893 .

Chiral HPLC: Chiralcel OD-H, 1.0\% isopropanol/hexanes, $1.0 \mathrm{ml} / \mathrm{min}, 76 \%$ ee
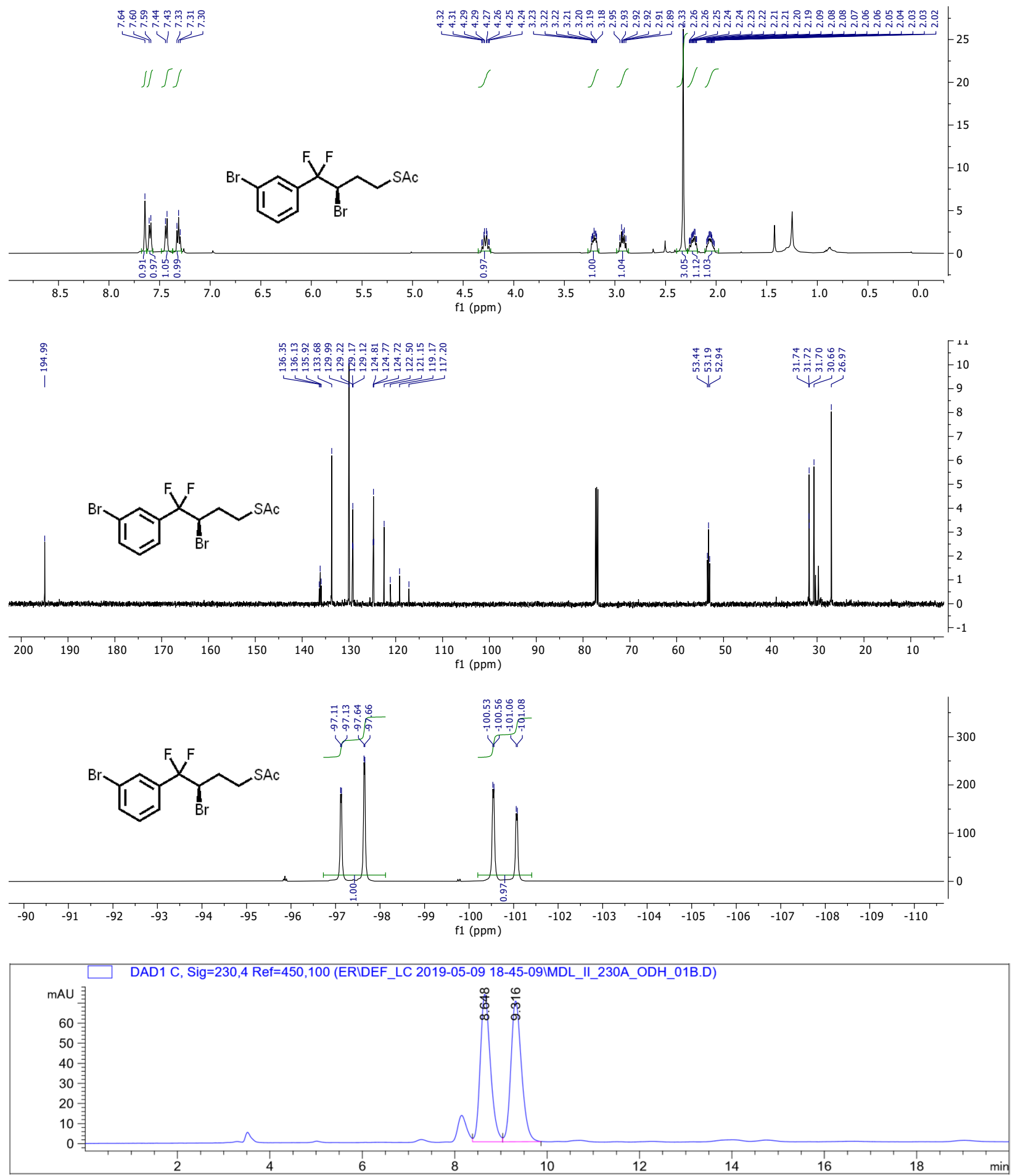

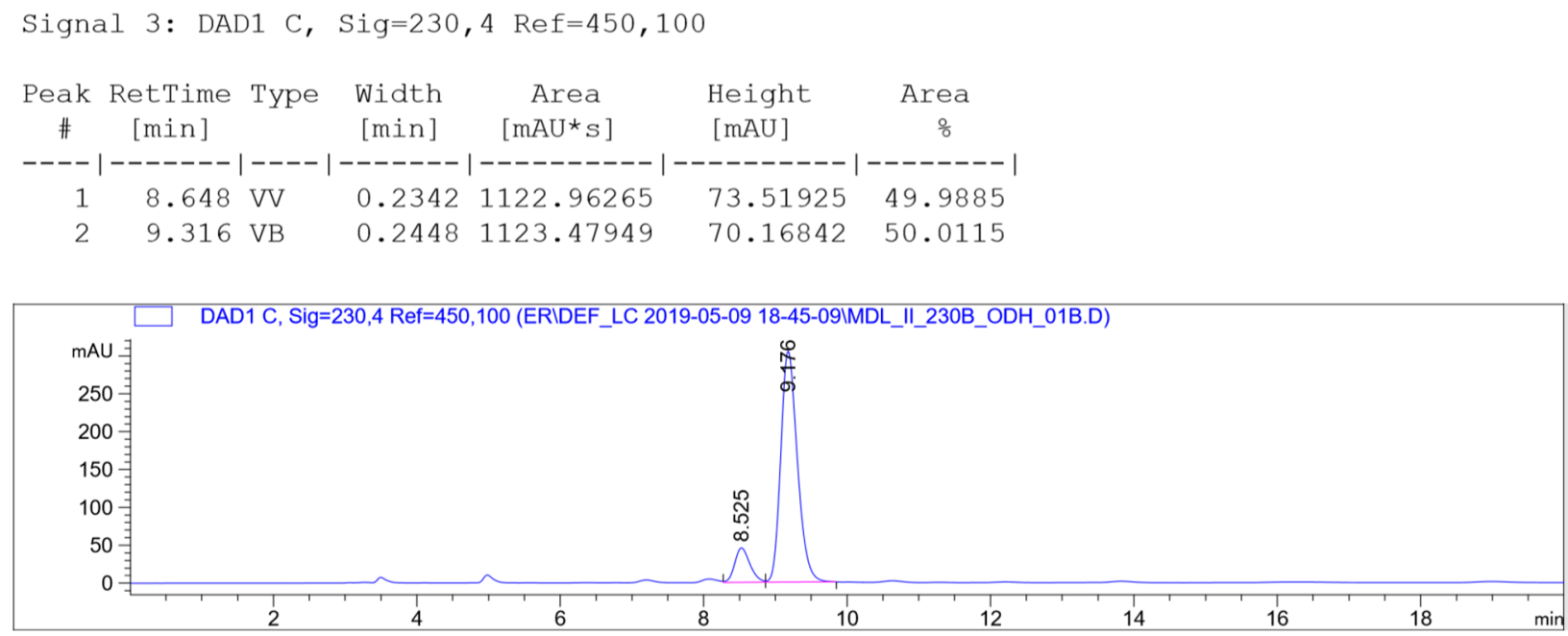

\begin{tabular}{|c|c|c|c|c|c|c|}
\hline $\begin{array}{c}\text { Peak } \\
\#\end{array}$ & $\begin{array}{c}\text { RetTime } \\
\quad \text { [min] }\end{array}$ & Type & $\begin{array}{l}\text { Width } \\
\text { [min] }\end{array}$ & $\begin{array}{c}\text { Area } \\
{\left[\mathrm{mAU}^{*} \mathrm{~s}\right]}\end{array}$ & $\begin{array}{l}\text { Height } \\
{[\mathrm{mAU}]}\end{array}$ & $\begin{array}{c}\text { Area } \\
\frac{\circ}{0}\end{array}$ \\
\hline 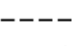 & |------ & & ------- & |---------- & ---------- & --------1 \\
\hline 1 & 8.525 & VV & 0.2268 & 676.37372 & 45.67154 & 12.0967 \\
\hline 2 & 9.176 & VB & 0.2478 & 4915.01953 & 305.29327 & 87.9033 \\
\hline
\end{tabular}

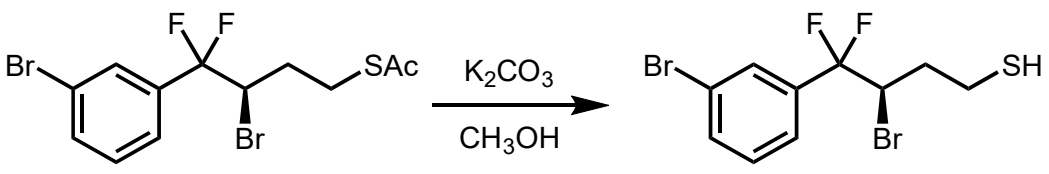

S3. (R)-3-bromo-4-(3-bromophenyl)-4,4-difluorobutane-1-thiol. To a solution of $\mathbf{S 2}$ (159 $\mathrm{mg}, 0.39 \mathrm{mmol}$ ) in methanol ( $8 \mathrm{~mL}$ ) under $\mathrm{N}_{2}$, was added solid $\mathrm{K}_{2} \mathrm{CO}_{3}(110 \mathrm{mg}$, 2 equiv). The mixture was briefly degassed by direct exposure to high vacuum, refilled with $\mathrm{N}_{2}$, and sealed. The mixture was stirred at room temperature for 1 hour and quenched with aqueous ammonium chloride. The aqueous layer was extracted $3 \mathrm{x}$ with $\mathrm{Et}_{2} \mathrm{O}$, and the combined organics dried over $\mathrm{MgSO}_{4}$, filtered, and concentrated. The residue was purified by column chromatography on $\mathrm{SiO}_{2}$ eluting with Hexanes/Et $\mathrm{Et}_{2} \mathrm{O}$ as a clear, colorless oil. (104 mg, $73 \%$ ).

${ }^{1} \mathrm{H}$ NMR (500 MHz, Chloroform- $d$ ) $\delta 7.67(\mathrm{t}, J=1.9 \mathrm{~Hz}, 1 \mathrm{H}), 7.61$ (dd, $\left.J=7.9,2.0 \mathrm{~Hz}, 1 \mathrm{H}\right), 7.46$ (dd, $J=7.9,1.6$ $\mathrm{Hz}, 1 \mathrm{H}), 7.33(\mathrm{t}, J=7.9 \mathrm{~Hz}, 1 \mathrm{H}), 4.56-4.45(\mathrm{~m}, 1 \mathrm{H}), 2.87$ (dtd, $J=13.7,7.1,4.4 \mathrm{~Hz}, 1 \mathrm{H}), 2.65$ (dtd, $J=13.6,9.1$, $6.8 \mathrm{~Hz}, 1 \mathrm{H}), 2.13(\mathrm{tdd}, J=9.4,7.4,4.5 \mathrm{~Hz}, 2 \mathrm{H}), 1.35(\mathrm{dd}, J=9.3,7.7 \mathrm{~Hz}, 1 \mathrm{H})$.

${ }^{13} \mathrm{C}$ NMR $(126 \mathrm{MHz}$, Chloroform- $d$ ) $\delta 136.33(\mathrm{t}, J=26.8 \mathrm{~Hz}), 133.69,130.04,129.15(\mathrm{t}, J=6.5 \mathrm{~Hz}), 124.72(\mathrm{t}, J=$ $6.1 \mathrm{~Hz}), 122.55,119.31(\mathrm{t}, J=247.9 \mathrm{~Hz}), 52.87(\mathrm{t}, J=31.5 \mathrm{~Hz}), 35.21$ (d, $J=2.3 \mathrm{~Hz}), 22.36$.

${ }^{19} \mathrm{~F}$ NMR (471 MHz, Chloroform- $d$ ) $\delta-97.99$ (dd, $\left.J=246.3,10.7 \mathrm{~Hz}, 1 \mathrm{~F}\right),-100.21$ (dd, $J=246.3,13.0 \mathrm{~Hz}, 1 \mathrm{~F}$ ). HRMS (EI): for $\mathrm{C}_{10} \mathrm{H}_{10} \mathrm{Br}_{2} \mathrm{~F}_{2} \mathrm{~S}$, [M] $]^{+}$calculated $\mathrm{m} / z=357.8833$ and 359.8812 and 361.8792 , found $\mathrm{m} / \mathrm{z}=357.8835$ and 359.8813 and 361.8792 . 

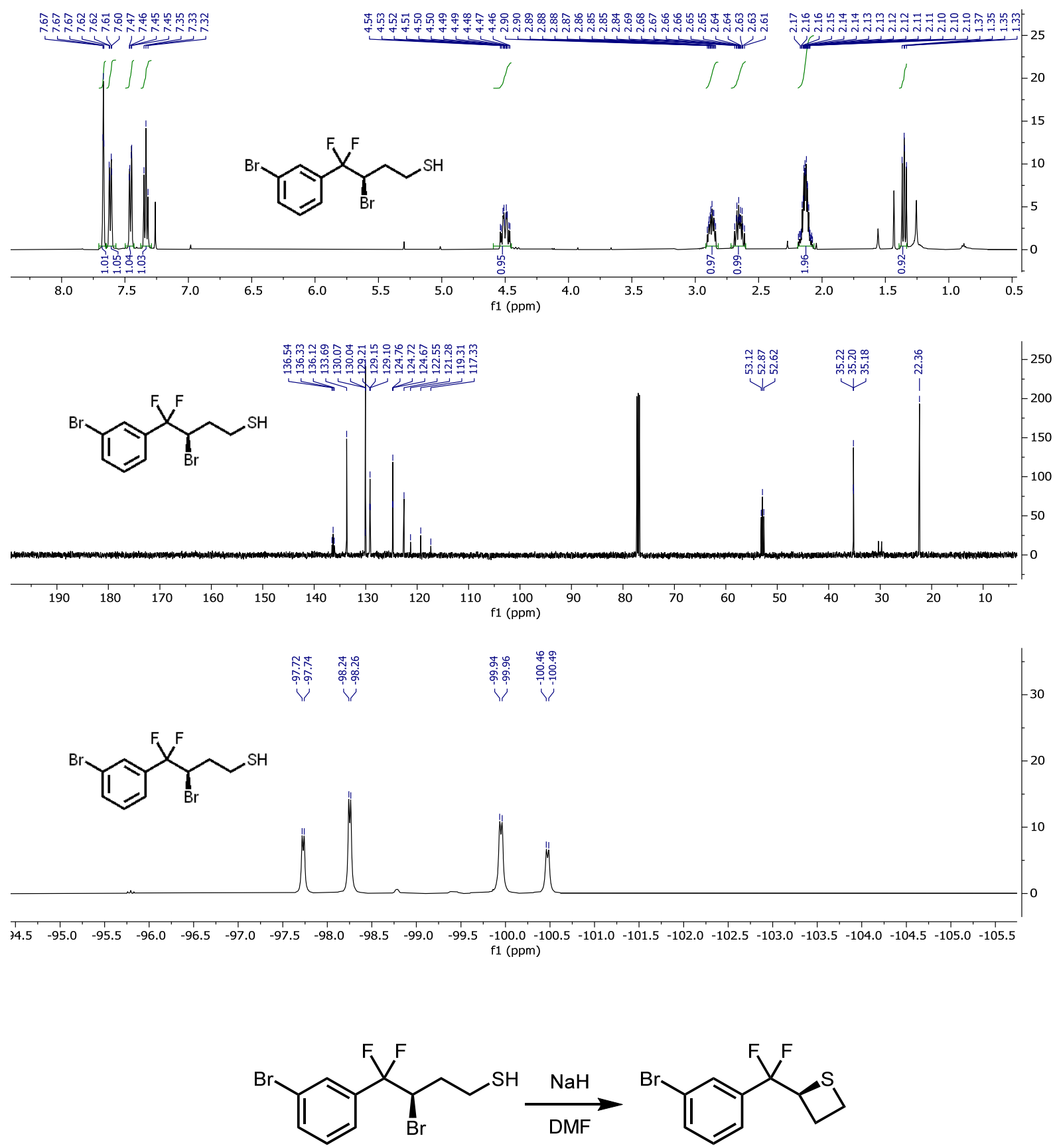

7. (S)-2-((3-bromophenyl)difluoromethyl)thietane. A solution of $\mathbf{S 3}(53 \mathrm{mg}, 0.147 \mathrm{mmol})$ in DMF (1.5 mL) was added under $\mathrm{N}_{2}$ to solid $\mathrm{NaH}(95 \%, 4.2 \mathrm{mg}, 1.2$ equiv) [Caution! Sodium hydride in DMF can lead to runaway exothermic reactions on large scale.]. The mixture was briefly degassed by direct exposure to high vacuum, refilled with $\mathrm{N}_{2}$, and sealed. The mixture was stirred at room $90{ }^{\circ} \mathrm{C}$ for 9 hours, and upon cooling to room temperature was quenched with aqueous ammonium chloride. The aqueous layer was extracted $3 \mathrm{x}$ with $\mathrm{Et}_{2} \mathrm{O}$, and the combined organics were washed three times with brine, dried over $\mathrm{MgSO}_{4}$, filtered, and concentrated. The residue was purified by column chromatography on $\mathrm{SiO}_{2}$ eluting with Hexanes/ $\mathrm{Et}_{2} \mathrm{O}$ as a clear, colorless oil (28 mg, $68 \%$ ).

${ }^{1} \mathrm{H}$ NMR (500 MHz, Chloroform- $d$ ) $\delta 7.66(\mathrm{t}, J=1.9 \mathrm{~Hz}, 1 \mathrm{H}), 7.61-7.52(\mathrm{~m}, 1 \mathrm{H}), 7.45(\mathrm{dt}, J=7.7,1.3 \mathrm{~Hz}, 1 \mathrm{H})$, $7.30(\mathrm{t}, J=7.9 \mathrm{~Hz}, 1 \mathrm{H}), 3.97$ (dddd, $J=15.4,11.1,7.7,6.6 \mathrm{~Hz}, 1 \mathrm{H}), 3.14-3.02(\mathrm{~m}, 2 \mathrm{H}), 3.02-2.94(\mathrm{~m}, 2 \mathrm{H})$. ${ }^{13} \mathrm{C}$ NMR (126 MHz, Chloroform- $d$ ) $\delta 136.61(\mathrm{t}, J=27.5 \mathrm{~Hz}), 133.30,129.99,128.87(\mathrm{t}, J=6.2 \mathrm{~Hz}), 124.36(\mathrm{t}, J=$ $5.9 \mathrm{~Hz}), 120.84$ (t, $J=208.4 \mathrm{~Hz}), 44.04(\mathrm{t}, J=31.1 \mathrm{~Hz}), 26.40$ (t, $J=3.5 \mathrm{~Hz}), 21.33$. 
${ }^{19} \mathrm{~F}$ NMR (471 MHz, Chloroform- $d$ ) $\delta-105.44$ (dd, $\left.J=244.1,11.1 \mathrm{~Hz}, 1 \mathrm{~F}\right),-108.25$ (dd, $J=244.1,15.5 \mathrm{~Hz}, 1 \mathrm{~F}$ ). HRMS (EI): for $\mathrm{C}_{10} \mathrm{H}_{9} \mathrm{BrF}_{2} \mathrm{~S}$, [M] $]^{+}$calculated $m / z=277.9571$ and 279.9550 , found $\mathrm{m} / z=277.9568$ and 279.9545 . Chiral GC: $\beta$-Cyclosil, isothermal $120^{\circ} \mathrm{C}, 14 \mathrm{psi}, 76 \%$ ee
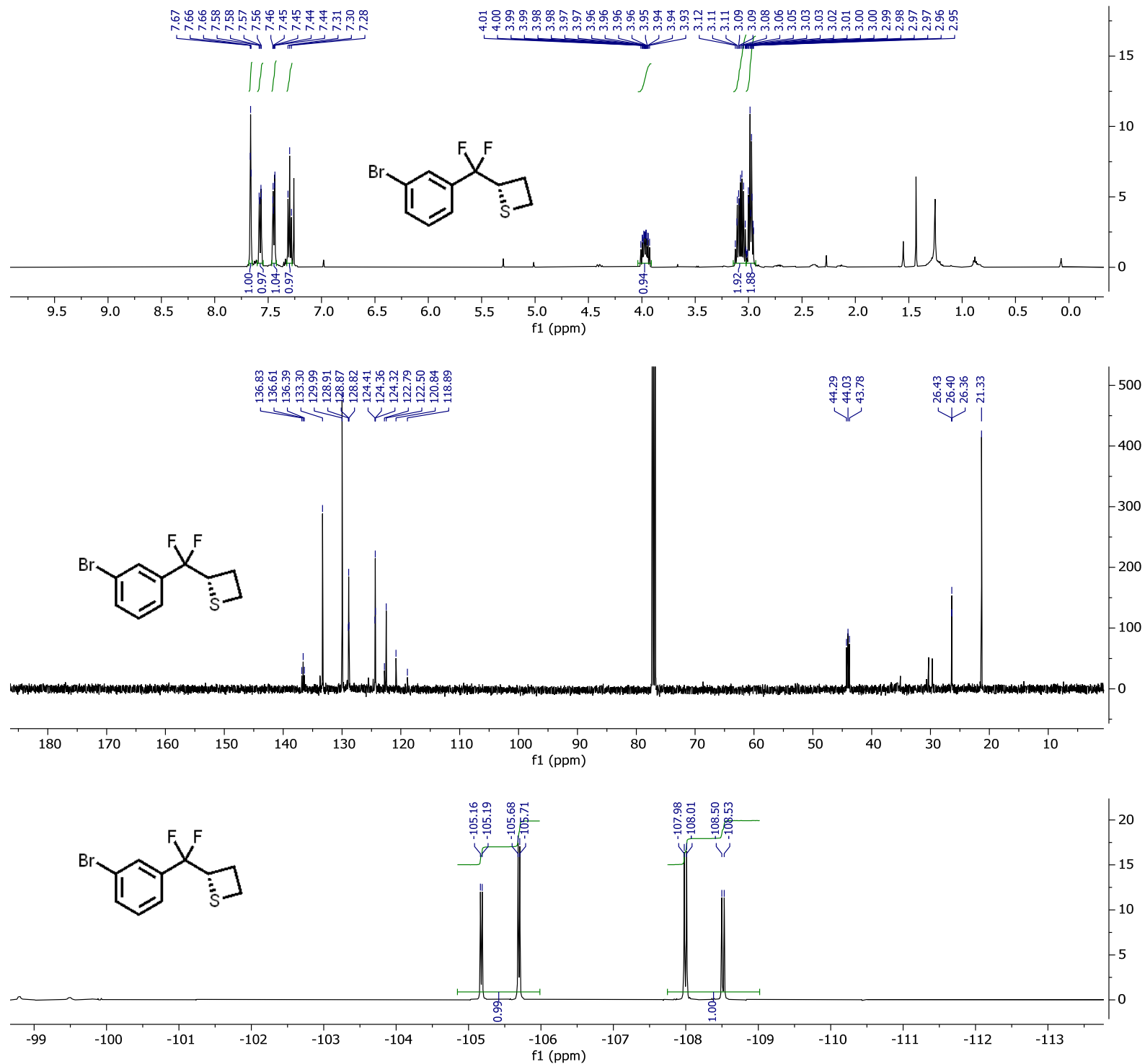

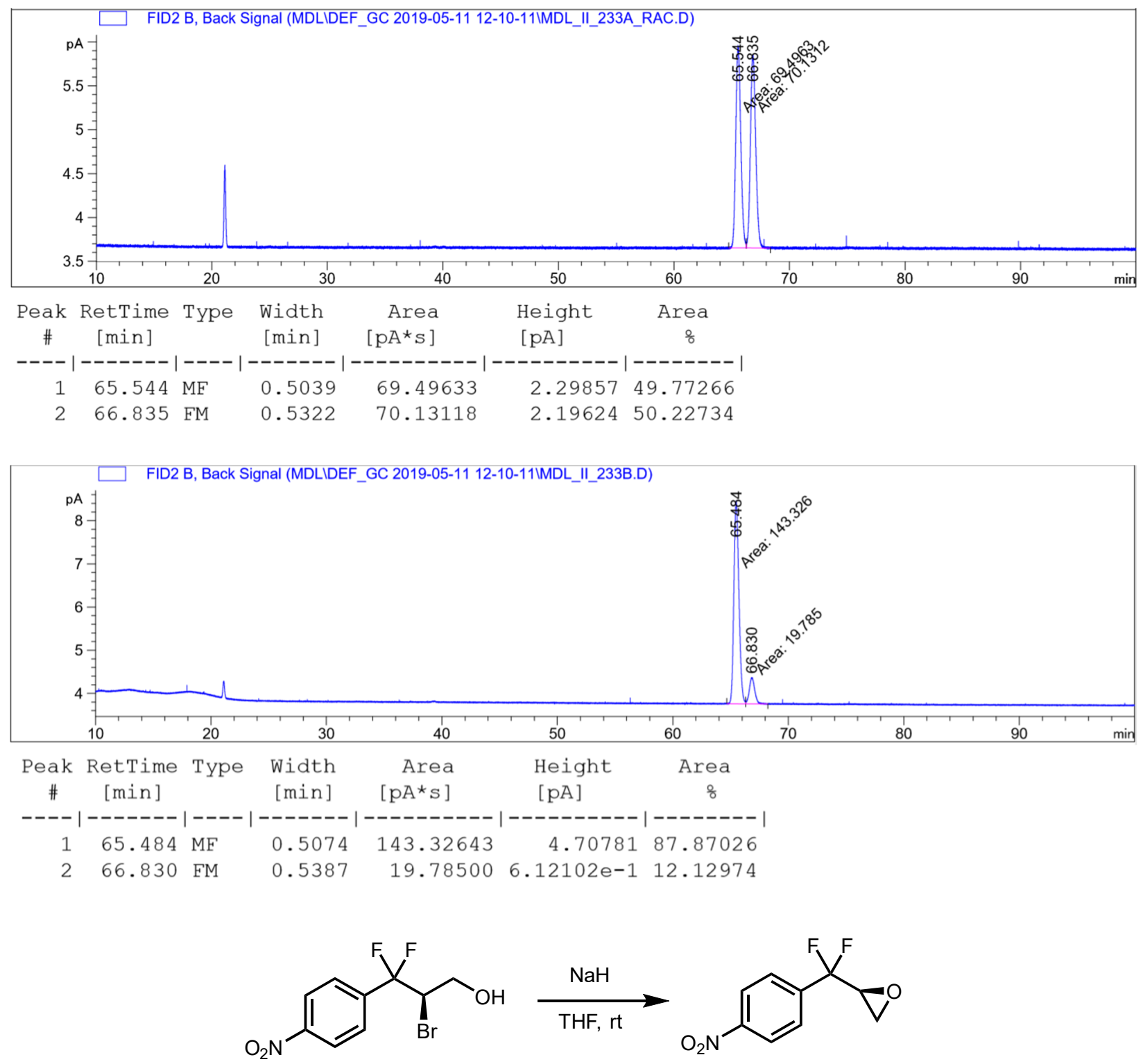

6. (S)-2-(difluoro(4-nitrophenyl)methyl)oxirane. A solution of $1 \mathrm{~s}(29.6 \mathrm{mg}, 0.1 \mathrm{mmol})$ in THF $(0.8 \mathrm{~mL})$ was added under $\mathrm{N}_{2}$ to a suspension of $\mathrm{NaH}(95 \%, 4.8 \mathrm{mg}, 2$ equiv) in THF $(0.2 \mathrm{~mL})$ at room temperature. The mixture was stirred for 15 minutes and was quenched by aqueous ammonium chloride. The aqueous layer was extracted $3 \mathrm{x}$ with $\mathrm{Et}_{2} \mathrm{O}$, and the combined organics dried over $\mathrm{Na}_{2} \mathrm{SO}_{4}$, filtered and concentrated. The residue was purified by column chromatography on $\mathrm{SiO}_{2}$ eluting with Hexanes/ $\mathrm{Et}_{2} \mathrm{O}$ and isolated as a white solid (13 mg, 70\%).

${ }^{1} \mathrm{H}$ NMR $\left(500 \mathrm{MHz}, \mathrm{CDCl}_{3}\right) \delta 8.30(\mathrm{~d}, J=8.2 \mathrm{~Hz}, 2 \mathrm{H}), 7.76-7.63(\mathrm{~m}, 2 \mathrm{H}), 3.46(\mathrm{dddd}, J=9.2,5.4,3.9,2.4 \mathrm{~Hz}$, $1 \mathrm{H}), 2.90(\mathrm{ddd}, J=5.3,3.6,1.6 \mathrm{~Hz}, 1 \mathrm{H}), 2.79(\mathrm{dd}, J=5.0,2.3 \mathrm{~Hz}, 1 \mathrm{H})$.

${ }^{13} \mathrm{C}$ NMR $\left(126 \mathrm{MHz}, \mathrm{CDCl}_{3}\right) \delta 149.28,139.69(\mathrm{t}, J=26.8 \mathrm{~Hz}), 127.06,123.69,118.05$ (dd, $\left.J=246.1,243.3 \mathrm{~Hz}\right)$, 52.24 (dd, $J=40.6,36.8 \mathrm{~Hz}$ ), 43.68 .

${ }^{19} \mathrm{~F}$ NMR $\left(471 \mathrm{MHz}, \mathrm{CDCl}_{3}\right) \delta-102.81(\mathrm{~d}, J=263.8 \mathrm{~Hz}, 1 \mathrm{~F}),-107.72(\mathrm{dd}, J=263.7,2.3 \mathrm{~Hz}, 1 \mathrm{~F})$.

HRMS (EI): for $\mathrm{C}_{9} \mathrm{H}_{7} \mathrm{~F}_{2} \mathrm{NO}_{3},[\mathrm{M}]^{+}$calculated $\mathrm{m} / \mathrm{z}=215.0389$, found $\mathrm{m} / \mathrm{z}=215.038$.

Chiral GC: $\beta$-Cyclosil, $40{ }^{\circ} \mathrm{C}$ to $120^{\circ} \mathrm{C}, 1{ }^{\circ} \mathrm{C} / \mathrm{min}, 14 \mathrm{psi}, 92 \%$ ee 

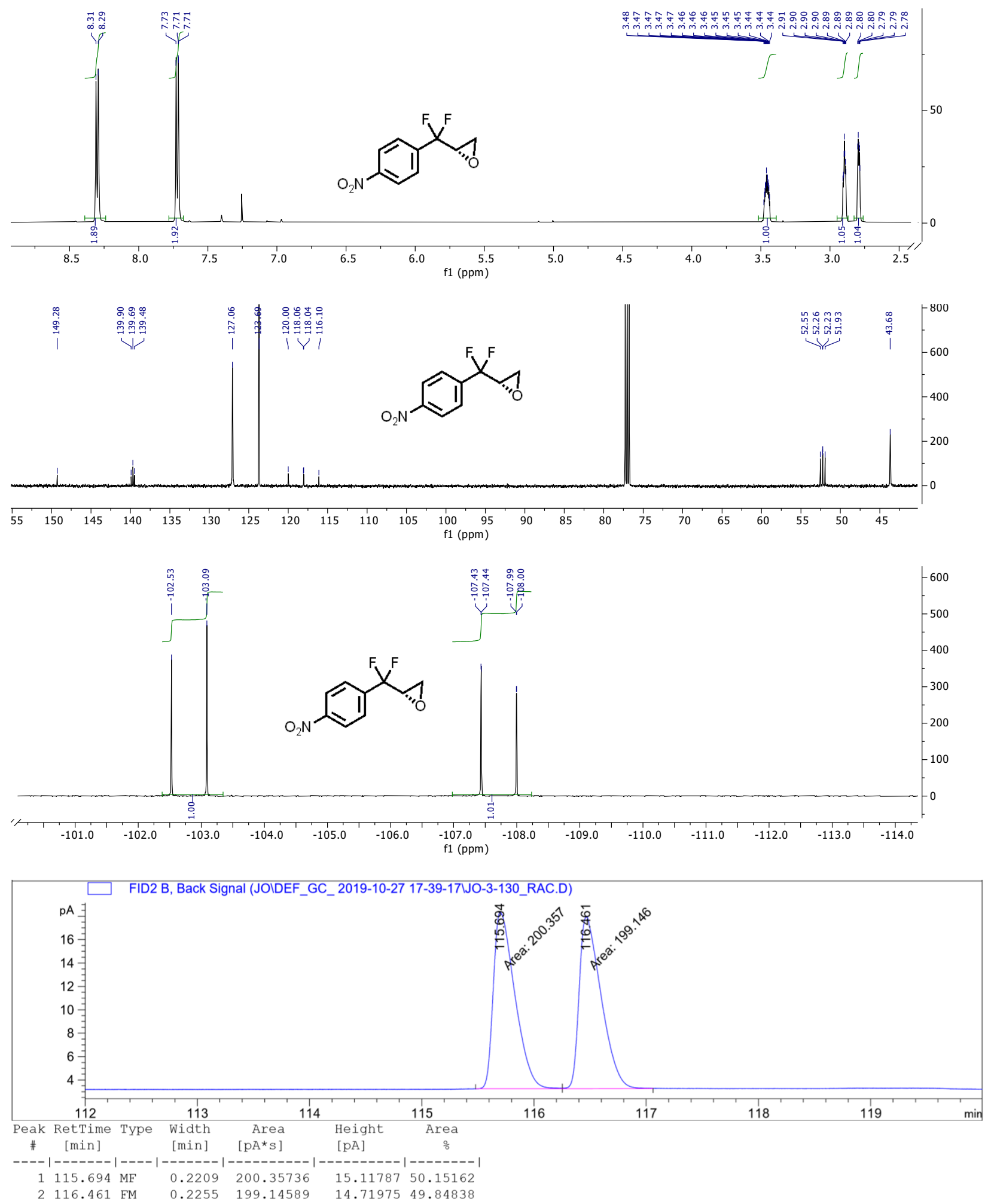


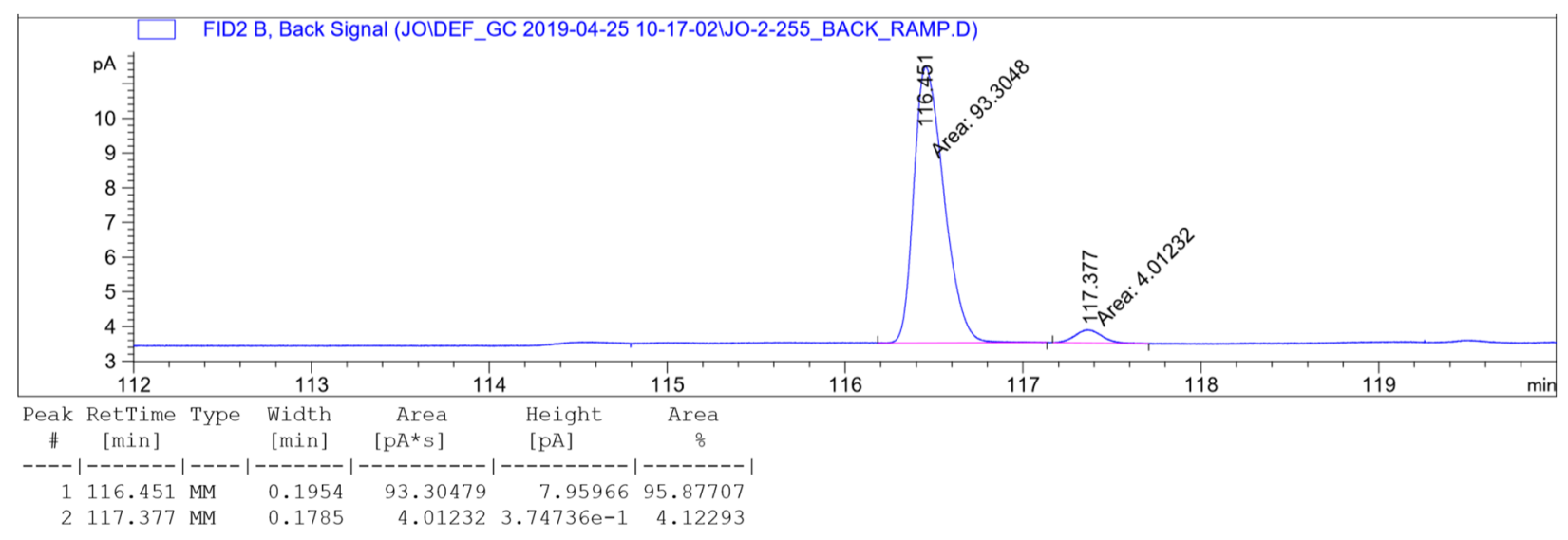


X-ray Crystallography: X-ray crystallography quality crystals were obtained of the following derivative (prepared by azide displacement using the method described for $\mathbf{5}$ on $\mathbf{1 i}$ followed by the standard click-chemistry technique with p-bromophenylacetylene) by vapor diffusion of pentane into MTBE:
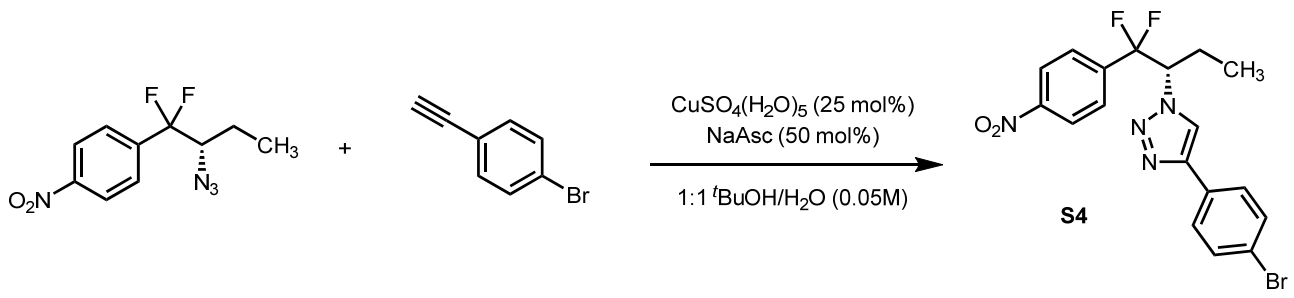

S4. (S)-4-(4-bromophenyl)-1-(1,1-difluoro-1-(4-nitrophenyl)butan-2-yl)-1H-1,2,3-triazole. The azide product (3 mg, $0.012 \mathrm{mmol}, 1$ equiv) was dissolved in a 1:1 mixture of $t \mathrm{BuOH} / \mathrm{H}_{2} \mathrm{O}(0.05 \mathrm{M}$ overall). To this solution was added, in succession, p-bromophenylacetylene ( $2.5 \mathrm{mg}, 0.014 \mathrm{mmol}, 1.2$ equiv), $\mathrm{CuSO}_{4} \cdot 5 \mathrm{H}_{2} \mathrm{O}(0.8 \mathrm{mg}, 0.003 \mathrm{mmol}, 0.25$ equiv), and sodium ascorbate (1.2 $\mathrm{mg}, 0.006 \mathrm{mmol}, 0.5$ equiv). The vial was placed under an $\mathrm{N}_{2}$ atmosphere and sealed with electrical tape. The solution was stirred overnight at room temperature. After this time, the reaction mixture was diluted with EtOAc and $\mathrm{H}_{2} \mathrm{O}$. The organic layers were separated and washed with brine, dried over $\mathrm{MgSO}_{4}$, filtered, and concentrated by rotary evaporation. The crude material was then purified by flash column chromatography (5\% to $60 \% \mathrm{Et}_{2} \mathrm{O} / \mathrm{Hexanes}$ ) to afford $\mathbf{S} 4$ as a white solid (5 $\mathrm{mg}, 95 \%$ yield).

X-ray crystallography: A crystal mounted on a diffractometer was collected data at $100 \mathrm{~K}$. The intensities of the reflections were collected by means of a Bruker APEX II CCD diffractometer (Мок $\alpha$ radiation, $\lambda=0.71073 \AA$ ), and equipped with an Oxford Cryosystems nitrogen flow apparatus. The collection method involved $0.5^{\circ}$ scans in $\omega$ at $28^{\circ}$ in $2 \theta$. Data integration down to $0.78 \AA$ resolution was carried out using SAINT V8.37A ${ }^{7}$ with reflection spot size optimization. Absorption corrections were made with the program SADABS. ${ }^{7}$ The structure was solved by the Intrinsic Phasing methods and refined by least-squares methods again $F^{2}$ using SHELXT-2014 ${ }^{8}$ and SHELXL-2014 ${ }^{9}$ with OLEX 2 interface. ${ }^{10}$ Non-hydrogen atoms were refined anisotropically, and hydrogen atoms were allowed to ride on the respective atoms. Crystal data as well as details of data collection and refinement are summarized in Table 1, and geometric parameters are shown in Table 2. The Ortep plots produced with SHELXL-2014 program, and the other drawings were produced with Accelrys DS Visualizer 2.0. ${ }^{11}$

\section{Table S1. Experimental details}

\begin{tabular}{|c|c|}
\hline & MLII-99-2 \\
\hline \multicolumn{2}{|l|}{ Crystal data } \\
\hline Chemical formula & $\mathrm{C}_{18} \mathrm{H}_{15} \mathrm{BrF}_{2} \mathrm{~N}_{4} \mathrm{O}_{2}$ \\
\hline$M_{\mathrm{r}}$ & 437.25 \\
\hline
\end{tabular}




\begin{tabular}{|c|c|}
\hline Crystal system, space group & Monoclinic, $P 2_{1}$ \\
\hline Temperature $(\mathrm{K})$ & 100 \\
\hline$a, b, c(\AA)$ & $12.6771(15), 5.6603(7), 13.2825(15)$ \\
\hline$\beta\left(^{\circ}\right)$ & $112.3222(18)$ \\
\hline$V\left(\AA^{3}\right)$ & $881.68(18)$ \\
\hline$Z$ & 2 \\
\hline Radiation type & Mo $K \alpha$ \\
\hline$\mu\left(\mathrm{mm}^{-1}\right)$ & 2.37 \\
\hline Crystal size $(\mathrm{mm})$ & $0.10 \times 0.04 \times 0.02$ \\
\hline \multicolumn{2}{|l|}{ Data collection } \\
\hline Diffractometer & Bruker D8 goniometer with CCD area detector \\
\hline Absorption correction & $\begin{array}{l}\text { Multi-scan } \\
\text { SADABS }\end{array}$ \\
\hline$T_{\min }, T_{\max }$ & $0.565,0.746$ \\
\hline $\begin{array}{l}\text { No. of measured, independent and } \\
\text { observed }[I>2 \sigma(I)] \text { reflections }\end{array}$ & $5582,3654,2999$ \\
\hline$R_{\text {int }}$ & 0.030 \\
\hline$(\sin \theta / \lambda)_{\max }\left(\AA^{-1}\right)$ & 0.641 \\
\hline \multicolumn{2}{|l|}{ Refinement } \\
\hline$R\left[F^{2}>2 \sigma\left(F^{2}\right)\right], w R\left(F^{2}\right), S$ & $0.051,0.119,1.03$ \\
\hline No. of reflections & 3654 \\
\hline No. of parameters & 245 \\
\hline No. of restraints & 1 \\
\hline H-atom treatment & H-atom parameters constrained \\
\hline$\Delta \rho_{\max }, \Delta \rho_{\min }\left(\mathrm{e} \AA^{-3}\right)$ & $1.13,-0.38$ \\
\hline Absolute structure & $\begin{array}{l}\text { Flack x determined using } 1028 \text { quotients [(I+)-(I-)]/[(I+)+(I-)] (Parsons, } \\
\text { Flack and Wagner, Acta Cryst. B69 (2013) 249-259). }\end{array}$ \\
\hline Absolute structure parameter & $-0.004(13)$ \\
\hline
\end{tabular}

Computer programs: SAINT 8.37A (Bruker-AXS, 2015), SHELXT2014 (Sheldrick, 2015), SHELXL2014

(Sheldrick, 2015), Bruker SHELXTL (Sheldrick, 2015).

Table S2. Geometric parameters $\left(\AA \AA{ }^{\circ}\right)$ 


\begin{tabular}{|c|c|c|c|}
\hline $\mathrm{Br} 1-\mathrm{C} 16$ & $1.908(7)$ & $\mathrm{C} 7-\mathrm{C} 8$ & $1.518(10)$ \\
\hline $\mathrm{F} 1-\mathrm{C} 7$ & $1.369(9)$ & $\mathrm{C} 8-\mathrm{C} 9$ & $1.529(10)$ \\
\hline $\mathrm{F} 2-\mathrm{C} 7$ & $1.373(8)$ & $\mathrm{C} 8-\mathrm{H} 8$ & 1.0000 \\
\hline $\mathrm{O} 1-\mathrm{N} 4$ & $1.253(9)$ & $\mathrm{C} 9-\mathrm{C} 10$ & $1.505(10)$ \\
\hline $\mathrm{O} 2-\mathrm{N} 4$ & $1.203(10)$ & C9-H9A & 0.9900 \\
\hline $\mathrm{N} 1-\mathrm{N} 2$ & $1.320(7)$ & C9-H9B & 0.9900 \\
\hline $\mathrm{N} 1-\mathrm{C} 12$ & $1.364(9)$ & $\mathrm{C} 10-\mathrm{H} 10 \mathrm{~A}$ & 0.9800 \\
\hline $\mathrm{N} 2-\mathrm{N} 3$ & $1.355(8)$ & $\mathrm{C} 10-\mathrm{H} 10 \mathrm{~B}$ & 0.9800 \\
\hline $\mathrm{N} 3-\mathrm{C} 11$ & $1.347(8)$ & $\mathrm{C} 10-\mathrm{H} 10 \mathrm{C}$ & 0.9800 \\
\hline N3- $\mathrm{C} 8$ & $1.475(8)$ & $\mathrm{C} 11-\mathrm{C} 12$ & $1.384(9)$ \\
\hline N4 $-\mathrm{C} 3$ & $1.480(8)$ & $\mathrm{C} 11-\mathrm{H} 11$ & 0.9500 \\
\hline $\mathrm{C} 1-\mathrm{C} 6$ & $1.384(10)$ & $\mathrm{C} 12-\mathrm{C} 13$ & $1.473(9)$ \\
\hline $\mathrm{C} 1-\mathrm{C} 2$ & $1.403(9)$ & $\mathrm{C} 13-\mathrm{C} 14$ & $1.393(12)$ \\
\hline $\mathrm{C} 1-\mathrm{H} 1$ & 0.9500 & $\mathrm{C} 13-\mathrm{C} 18$ & $1.396(9)$ \\
\hline $\mathrm{C} 2-\mathrm{C} 3$ & $1.379(10)$ & $\mathrm{C} 14-\mathrm{C} 15$ & $1.384(9)$ \\
\hline $\mathrm{C} 2-\mathrm{H} 2$ & 0.9500 & $\mathrm{C} 14-\mathrm{H} 14$ & 0.9500 \\
\hline $\mathrm{C} 3-\mathrm{C} 4$ & $1.381(9)$ & $\mathrm{C} 15-\mathrm{C} 16$ & $1.374(10)$ \\
\hline $\mathrm{C} 4-\mathrm{C} 5$ & $1.379(9)$ & $\mathrm{C} 15-\mathrm{H} 15$ & 0.9500 \\
\hline $\mathrm{C} 4-\mathrm{H} 4$ & 0.9500 & $\mathrm{C} 16-\mathrm{C} 17$ & $1.371(10)$ \\
\hline $\mathrm{C} 5-\mathrm{C} 6$ & $1.403(12)$ & $\mathrm{C} 17-\mathrm{C} 18$ & $1.387(9)$ \\
\hline $\mathrm{C} 5-\mathrm{H} 5$ & 0.9500 & $\mathrm{C} 17-\mathrm{H} 17$ & 0.9500 \\
\hline $\mathrm{C} 6-\mathrm{C} 7$ & $1.524(10)$ & $\mathrm{C} 18-\mathrm{H} 18$ & 0.9500 \\
\hline $\mathrm{N} 2-\mathrm{N} 1-\mathrm{C} 12$ & $108.9(6)$ & $\mathrm{C} 9-\mathrm{C} 8-\mathrm{H} 8$ & 107.9 \\
\hline $\mathrm{N} 1-\mathrm{N} 2-\mathrm{N} 3$ & $106.8(6)$ & $\mathrm{C} 10-\mathrm{C} 9-\mathrm{C} 8$ & $113.9(6)$ \\
\hline $\mathrm{C} 11-\mathrm{N} 3-\mathrm{N} 2$ & $111.6(5)$ & $\mathrm{C} 10-\mathrm{C} 9-\mathrm{H} 9 \mathrm{~A}$ & 108.8 \\
\hline $\mathrm{C} 11-\mathrm{N} 3-\mathrm{C} 8$ & $128.7(6)$ & $\mathrm{C} 8-\mathrm{C} 9-\mathrm{H} 9 \mathrm{~A}$ & 108.8 \\
\hline $\mathrm{N} 2-\mathrm{N} 3-\mathrm{C} 8$ & $119.6(5)$ & $\mathrm{C} 10-\mathrm{C} 9-\mathrm{H} 9 \mathrm{~B}$ & 108.8 \\
\hline $\mathrm{O} 2-\mathrm{N} 4-\mathrm{O} 1$ & $124.2(6)$ & C8- $89-\mathrm{H} 9 \mathrm{~B}$ & 108.8 \\
\hline $\mathrm{O} 2-\mathrm{N} 4-\mathrm{C} 3$ & $119.2(7)$ & $\mathrm{H} 9 \mathrm{~A}-\mathrm{C} 9-\mathrm{H} 9 \mathrm{~B}$ & 107.7 \\
\hline $\mathrm{O} 1-\mathrm{N} 4-\mathrm{C} 3$ & $116.6(8)$ & $\mathrm{C} 9-\mathrm{C} 10-\mathrm{H} 10 \mathrm{~A}$ & 109.5 \\
\hline $\mathrm{C} 6-\mathrm{C} 1-\mathrm{C} 2$ & $120.0(6)$ & $\mathrm{C} 9-\mathrm{C} 10-\mathrm{H} 10 \mathrm{~B}$ & 109.5 \\
\hline $\mathrm{C} 6-\mathrm{C} 1-\mathrm{H} 1$ & 120.0 & $\mathrm{H} 10 \mathrm{~A}-\mathrm{C} 10-\mathrm{H} 10 \mathrm{~B}$ & 109.5 \\
\hline $\mathrm{C} 2-\mathrm{C} 1-\mathrm{H} 1$ & 120.0 & $\mathrm{C} 9-\mathrm{C} 10-\mathrm{H} 10 \mathrm{C}$ & 109.5 \\
\hline
\end{tabular}




\begin{tabular}{|c|c|c|c|}
\hline $\mathrm{C} 3-\mathrm{C} 2-\mathrm{C} 1$ & $117.4(6)$ & $\mathrm{H} 10 \mathrm{~A}-\mathrm{C} 10-\mathrm{H} 10 \mathrm{C}$ & 109.5 \\
\hline $\mathrm{C} 3-\mathrm{C} 2-\mathrm{H} 2$ & 121.3 & $\mathrm{H} 10 \mathrm{~B}-\mathrm{C} 10-\mathrm{H} 10 \mathrm{C}$ & 109.5 \\
\hline $\mathrm{C} 1-\mathrm{C} 2-\mathrm{H} 2$ & 121.3 & $\mathrm{~N} 3-\mathrm{C} 11-\mathrm{C} 12$ & $104.1(6)$ \\
\hline $\mathrm{C} 2-\mathrm{C} 3-\mathrm{C} 4$ & $123.5(7)$ & $\mathrm{N} 3-\mathrm{C} 11-\mathrm{H} 11$ & 127.9 \\
\hline $\mathrm{C} 2-\mathrm{C} 3-\mathrm{N} 4$ & $119.2(7)$ & $\mathrm{C} 12-\mathrm{C} 11-\mathrm{H} 11$ & 127.9 \\
\hline $\mathrm{C} 4-\mathrm{C} 3-\mathrm{N} 4$ & $117.3(6)$ & $\mathrm{N} 1-\mathrm{C} 12-\mathrm{C} 11$ & $108.6(6)$ \\
\hline $\mathrm{C} 5-\mathrm{C} 4-\mathrm{C} 3$ & $119.0(7)$ & $\mathrm{N} 1-\mathrm{C} 12-\mathrm{C} 13$ & $122.7(6)$ \\
\hline $\mathrm{C} 5-\mathrm{C} 4-\mathrm{H} 4$ & 120.5 & $\mathrm{C} 11-\mathrm{C} 12-\mathrm{C} 13$ & $128.7(6)$ \\
\hline $\mathrm{C} 3-\mathrm{C} 4-\mathrm{H} 4$ & 120.5 & $\mathrm{C} 14-\mathrm{C} 13-\mathrm{C} 18$ & $118.7(6)$ \\
\hline $\mathrm{C} 4-\mathrm{C} 5-\mathrm{C} 6$ & $119.1(7)$ & $\mathrm{C} 14-\mathrm{C} 13-\mathrm{C} 12$ & $120.7(6)$ \\
\hline $\mathrm{C} 4-\mathrm{C} 5-\mathrm{H} 5$ & 120.5 & $\mathrm{C} 18-\mathrm{C} 13-\mathrm{C} 12$ & $120.7(6)$ \\
\hline $\mathrm{C} 6-\mathrm{C} 5-\mathrm{H} 5$ & 120.5 & $\mathrm{C} 15-\mathrm{C} 14-\mathrm{C} 13$ & $120.8(7)$ \\
\hline $\mathrm{C} 1-\mathrm{C} 6-\mathrm{C} 5$ & $121.0(6)$ & $\mathrm{C} 15-\mathrm{C} 14-\mathrm{H} 14$ & 119.6 \\
\hline $\mathrm{C} 1-\mathrm{C} 6-\mathrm{C} 7$ & $118.5(6)$ & $\mathrm{C} 13-\mathrm{C} 14-\mathrm{H} 14$ & 119.6 \\
\hline $\mathrm{C} 5-\mathrm{C} 6-\mathrm{C} 7$ & $120.5(6)$ & $\mathrm{C} 16-\mathrm{C} 15-\mathrm{C} 14$ & $119.1(7)$ \\
\hline $\mathrm{F} 1-\mathrm{C} 7-\mathrm{F} 2$ & $105.1(5)$ & $\mathrm{C} 16-\mathrm{C} 15-\mathrm{H} 15$ & 120.4 \\
\hline $\mathrm{F} 1-\mathrm{C} 7-\mathrm{C} 8$ & $109.8(6)$ & $\mathrm{C} 14-\mathrm{C} 15-\mathrm{H} 15$ & 120.4 \\
\hline $\mathrm{F} 2-\mathrm{C} 7-\mathrm{C} 8$ & $109.4(5)$ & $\mathrm{C} 17-\mathrm{C} 16-\mathrm{C} 15$ & $121.6(6)$ \\
\hline $\mathrm{F} 1-\mathrm{C} 7-\mathrm{C} 6$ & $108.8(5)$ & $\mathrm{C} 17-\mathrm{C} 16-\mathrm{Br} 1$ & $120.5(6)$ \\
\hline $\mathrm{F} 2-\mathrm{C} 7-\mathrm{C} 6$ & $109.5(6)$ & $\mathrm{C} 15-\mathrm{C} 16-\mathrm{Br} 1$ & $118.0(5)$ \\
\hline $\mathrm{C} 8-\mathrm{C} 7-\mathrm{C} 6$ & $113.8(6)$ & $\mathrm{C} 16-\mathrm{C} 17-\mathrm{C} 18$ & $119.4(7)$ \\
\hline $\mathrm{N} 3-\mathrm{C} 8-\mathrm{C} 7$ & $108.6(6)$ & $\mathrm{C} 16-\mathrm{C} 17-\mathrm{H} 17$ & 120.3 \\
\hline $\mathrm{N} 3-\mathrm{C} 8-\mathrm{C} 9$ & $110.9(5)$ & $\mathrm{C} 18-\mathrm{C} 17-\mathrm{H} 17$ & 120.3 \\
\hline $\mathrm{C} 7-\mathrm{C} 8-\mathrm{C} 9$ & $113.5(6)$ & $\mathrm{C} 17-\mathrm{C} 18-\mathrm{C} 13$ & $120.4(6)$ \\
\hline $\mathrm{N} 3-\mathrm{C} 8-\mathrm{H} 8$ & 107.9 & $\mathrm{C} 17-\mathrm{C} 18-\mathrm{H} 18$ & 119.8 \\
\hline $\mathrm{C} 7-\mathrm{C} 8-\mathrm{H} 8$ & 107.9 & $\mathrm{C} 13-\mathrm{C} 18-\mathrm{H} 18$ & 119.8 \\
\hline $\mathrm{C} 12-\mathrm{N} 1-\mathrm{N} 2-\mathrm{N} 3$ & $-0.3(7)$ & $\mathrm{F} 2-\mathrm{C} 7-\mathrm{C} 8-\mathrm{N} 3$ & $-52.8(7)$ \\
\hline $\mathrm{N} 1-\mathrm{N} 2-\mathrm{N} 3-\mathrm{C} 11$ & $0.9(7)$ & $\mathrm{C} 6-\mathrm{C} 7-\mathrm{C} 8-\mathrm{N} 3$ & $-175.7(6)$ \\
\hline $\mathrm{N} 1-\mathrm{N} 2-\mathrm{N} 3-\mathrm{C} 8$ & $179.4(5)$ & $\mathrm{F} 1-\mathrm{C} 7-\mathrm{C} 8-\mathrm{C} 9$ & $-174.1(5)$ \\
\hline $\mathrm{C} 6-\mathrm{C} 1-\mathrm{C} 2-\mathrm{C} 3$ & $0.9(10)$ & $\mathrm{F} 2-\mathrm{C} 7-\mathrm{C} 8-\mathrm{C} 9$ & $71.0(7)$ \\
\hline $\mathrm{C} 1-\mathrm{C} 2-\mathrm{C} 3-\mathrm{C} 4$ & $1.6(10)$ & $\mathrm{C} 6-\mathrm{C} 7-\mathrm{C} 8-\mathrm{C} 9$ & $-51.9(8)$ \\
\hline $\mathrm{C} 1-\mathrm{C} 2-\mathrm{C} 3-\mathrm{N} 4$ & $-177.5(6)$ & $\mathrm{N} 3-\mathrm{C} 8-\mathrm{C} 9-\mathrm{C} 10$ & $-59.4(8)$ \\
\hline $\mathrm{O} 2-\mathrm{N} 4-\mathrm{C} 3-\mathrm{C} 2$ & $169.8(7)$ & $\mathrm{C} 7-\mathrm{C} 8-\mathrm{C} 9-\mathrm{C} 10$ & $177.9(6)$ \\
\hline
\end{tabular}




\begin{tabular}{|c|c|c|c|}
\hline $\mathrm{O} 1-\mathrm{N} 4-\mathrm{C} 3-\mathrm{C} 2$ & $-7.8(9)$ & $\mathrm{N} 2-\mathrm{N} 3-\mathrm{C} 11-\mathrm{C} 12$ & $-1.1(7)$ \\
\hline $\mathrm{O} 2-\mathrm{N} 4-\mathrm{C} 3-\mathrm{C} 4$ & $-9.4(9)$ & $\mathrm{C} 8-\mathrm{N} 3-\mathrm{C} 11-\mathrm{C} 12$ & $-179.4(6)$ \\
\hline $\mathrm{O} 1-\mathrm{N} 4-\mathrm{C} 3-\mathrm{C} 4$ & $172.9(6)$ & $\mathrm{N} 2-\mathrm{N} 1-\mathrm{C} 12-\mathrm{C} 11$ & $-0.3(7)$ \\
\hline $\mathrm{C} 2-\mathrm{C} 3-\mathrm{C} 4-\mathrm{C} 5$ & $-2.4(10)$ & $\mathrm{N} 2-\mathrm{N} 1-\mathrm{C} 12-\mathrm{C} 13$ & $-179.9(6)$ \\
\hline $\mathrm{N} 4-\mathrm{C} 3-\mathrm{C} 4-\mathrm{C} 5$ & $176.8(6)$ & $\mathrm{N} 3-\mathrm{C} 11-\mathrm{C} 12-\mathrm{N} 1$ & $0.9(7)$ \\
\hline $\mathrm{C} 3-\mathrm{C} 4-\mathrm{C} 5-\mathrm{C} 6$ & $0.6(10)$ & $\mathrm{N} 3-\mathrm{C} 11-\mathrm{C} 12-\mathrm{C} 13$ & $-179.6(6)$ \\
\hline $\mathrm{C} 2-\mathrm{C} 1-\mathrm{C} 6-\mathrm{C} 5$ & $-2.6(10)$ & $\mathrm{N} 1-\mathrm{C} 12-\mathrm{C} 13-\mathrm{C} 14$ & $160.4(6)$ \\
\hline $\mathrm{C} 2-\mathrm{C} 1-\mathrm{C} 6-\mathrm{C} 7$ & $177.6(6)$ & $\mathrm{C} 11-\mathrm{C} 12-\mathrm{C} 13-\mathrm{C} 14$ & $-19.1(10)$ \\
\hline $\mathrm{C} 4-\mathrm{C} 5-\mathrm{C} 6-\mathrm{C} 1$ & $1.9(10)$ & $\mathrm{N} 1-\mathrm{C} 12-\mathrm{C} 13-\mathrm{C} 18$ & $-19.8(10)$ \\
\hline $\mathrm{C} 4-\mathrm{C} 5-\mathrm{C} 6-\mathrm{C} 7$ & $-178.3(6)$ & $\mathrm{C} 11-\mathrm{C} 12-\mathrm{C} 13-\mathrm{C} 18$ & $160.7(7)$ \\
\hline $\mathrm{C} 1-\mathrm{C} 6-\mathrm{C} 7-\mathrm{F} 1$ & $52.7(8)$ & $\mathrm{C} 18-\mathrm{C} 13-\mathrm{C} 14-\mathrm{C} 15$ & $1.7(9)$ \\
\hline $\mathrm{C} 5-\mathrm{C} 6-\mathrm{C} 7-\mathrm{F} 1$ & $-127.0(7)$ & $\mathrm{C} 12-\mathrm{C} 13-\mathrm{C} 14-\mathrm{C} 15$ & $-178.5(6)$ \\
\hline $\mathrm{C} 1-\mathrm{C} 6-\mathrm{C} 7-\mathrm{F} 2$ & $167.2(6)$ & $\mathrm{C} 13-\mathrm{C} 14-\mathrm{C} 15-\mathrm{C} 16$ & $-0.7(10)$ \\
\hline $\mathrm{C} 5-\mathrm{C} 6-\mathrm{C} 7-\mathrm{F} 2$ & $-12.6(9)$ & $\mathrm{C} 14-\mathrm{C} 15-\mathrm{C} 16-\mathrm{C} 17$ & $-0.4(10)$ \\
\hline $\mathrm{C} 1-\mathrm{C} 6-\mathrm{C} 7-\mathrm{C} 8$ & $-70.0(8)$ & $\mathrm{C} 14-\mathrm{C} 15-\mathrm{C} 16-\mathrm{Br} 1$ & $179.5(5)$ \\
\hline $\mathrm{C} 5-\mathrm{C} 6-\mathrm{C} 7-\mathrm{C} 8$ & $110.2(7)$ & $\mathrm{C} 15-\mathrm{C} 16-\mathrm{C} 17-\mathrm{C} 18$ & $0.5(10)$ \\
\hline $\mathrm{C} 11-\mathrm{N} 3-\mathrm{C} 8-\mathrm{C} 7$ & $86.8(8)$ & $\mathrm{Br} 1-\mathrm{C} 16-\mathrm{C} 17-\mathrm{C} 18$ & $-179.5(5)$ \\
\hline $\mathrm{N} 2-\mathrm{N} 3-\mathrm{C} 8-\mathrm{C} 7$ & $-91.4(7)$ & $\mathrm{C} 16-\mathrm{C} 17-\mathrm{C} 18-\mathrm{C} 13$ & $0.6(10)$ \\
\hline $\mathrm{C} 11-\mathrm{N} 3-\mathrm{C} 8-\mathrm{C} 9$ & $-38.6(10)$ & $\mathrm{C} 14-\mathrm{C} 13-\mathrm{C} 18-\mathrm{C} 17$ & $-1.7(9)$ \\
\hline $\mathrm{N} 2-\mathrm{N} 3-\mathrm{C} 8-\mathrm{C} 9$ & $143.3(6)$ & $\mathrm{C} 12-\mathrm{C} 13-\mathrm{C} 18-\mathrm{C} 17$ & $178.5(6)$ \\
\hline $\mathrm{F} 1-\mathrm{C} 7-\mathrm{C} 8-\mathrm{N} 3$ & $62.1(7)$ & & \\
\hline
\end{tabular}

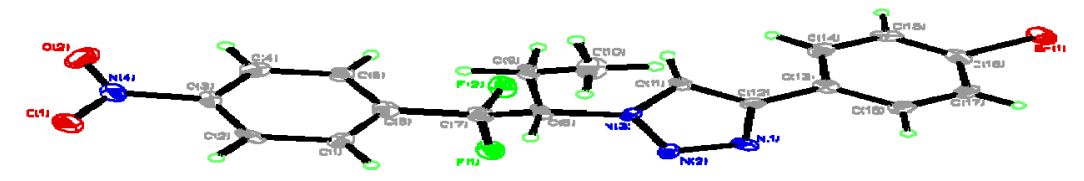

Figure S9. Perspective views showing 50\% probability displacement 


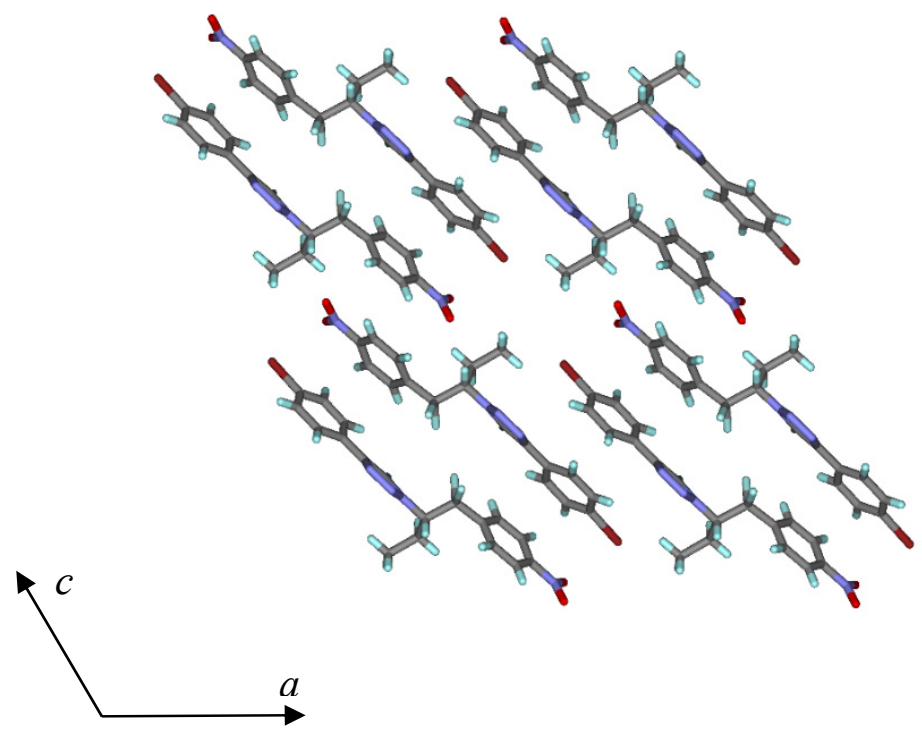

Figure S10. Three-dimensional supramolecular architecture viewed along the $b$-axis direction. 


\section{References:}

1) Jobin-Des Lauriers, A.; Legault, C. Y. Iodine(III)-Mediated Oxidative Hydrolysis of Haloalkenes: Access to $\alpha$-Halo Ketones by a Release-and-Catch Mechanism. Org. Lett. 2016, 18, 108-111.

2) Paraja, M.; Barroso, R.; Cabal, M. P.; Valdés, C. Synthesis of Highly Substituted Polyenes by PalladiumCatalyzed Cross-Couplings of Sterically Encumbered Alkenyl Bromides and N-Tosylhydrazones. $A d v$. Synth. Catal. 2017, 359, 1058-1062.

3) Shunatona, H. P.; Fruh, N.; Wang, Y.-M.; Rauniyar, V.; Toste, F. D. Enantioselective Fluoroamination: 1,4-Addition to Conjugated Dienes Using Anionic Phase-Transfer Catalysis. Angew. Chem. Int. Ed. 2013, 52, 7724-7727.

4) Murphy, S. K.; Dong, V. M. Enantioselective Ketone Hydroacylation Using Noyori's Transfer Hydrogenation Catalyst. J. Am. Chem. Soc. 2013, 135, 5553-5556.

5) Banik, S. M.; Medley, J. W.; Jacobsen, E. N. Catalytic, asymmetric difluorination of alkenes to generate difluoromethylated stereocenters. Science 2016, 353, 51-54.

6) Commercial $m$ CPBA ( $77 \%$ by weight) was found to give irreproducible results. Purified $m$ CPBA was found to give consistent yields and selectivities. $m$ CPBA was purified by the standard method: Schwartz, N. N., Blumbergs, J. H. Epoxidations with m-Chloroperbenzoic Acid. J. Org. Chem. 1964, 29, 1976-1979.

7) Bruker AXS APEX3, Bruker AXS, Madison, Wisconsin, 2016.

8) Dolomanov, O. V.; Bourhis, L. J.; Gildea, R. J.; Howard, J. A. K.; Puschmann H. OLEX2: a complete structure solution, refinement and analysis program. J. Appl. Cryst. 2009, 42, 339-34.

9) Sheldrick, G. M. SHELXT - Integrated space-group and crystal-structure determination. Acta Cryst. 2015. A71, 3-8.

10) Sheldrick, G. M. Crystal structure refinement with SHELXL. Acta Cryst. 2015, C71, 3-8.

11) Accelrys DS Visualizer v2.0.1, Accelrys Software. Inc., 2007. 